Portland State University

PDXScholar

$12-7-2020$

\title{
Achieving High Reliability Organizations Using Fuzzy Cognitive Maps - the Case of Offshore Oil and Gas
}

Ahmed A. Alibage

Portland State University

Follow this and additional works at: https://pdxscholar.library.pdx.edu/open_access_etds

Part of the Engineering Commons

Let us know how access to this document benefits you.

\section{Recommended Citation}

Alibage, Ahmed A., "Achieving High Reliability Organizations Using Fuzzy Cognitive Maps - the Case of Offshore Oil and Gas" (2020). Dissertations and Theses. Paper 5606.

https://doi.org/10.15760/etd.7478

This Dissertation is brought to you for free and open access. It has been accepted for inclusion in Dissertations and Theses by an authorized administrator of PDXScholar. Please contact us if we can make this document more accessible: pdxscholar@pdx.edu. 
Achieving High Reliability Organizations Using Fuzzy Cognitive Maps: The Case of Offshore Oil and Gas

by

Ahmed A. Alibage

A dissertation submitted in partial fulfillment of the requirements for the degree of

Doctor of Philosophy

in

Technology Management

Dissertation Committee:

Antonie Jetter, Chair

Wayne Wakeland

Charles Weber

Steven Scyphers

Portland State University

2020 
(C) 2020 Ahmed A. Alibage 


\begin{abstract}
The safety culture of so-called high-reliability organizations (HROs) encompasses values, routines, and work processes that allow an organization to prevent mistakes and quickly bounce back if unexpected events occur. It is said to provide a model for improving organizational resilience in the offshore oil and gas industry, where small errors can grow into accidents with devastating environmental, social, and economic impacts. To date, such a transfer of successful practices is impeded by a lack of system perspective that would allow researchers and practitioners to fully understand the safety dynamics in HROs, adjust them to the unique setting of offshore oil and gas, plan safety interventions, and anticipate the direct and indirect effects of these interventions.

In this dissertation, I have developed and rigorously tested a model of how safety interventions impact the interdependent aspects of the HROs' characteristics, based on peer-reviewed research, an industry workshop, and a survey of offshore oil and gas practitioners. This approach combines the qualitative research methods of Thematic Analysis (TA) and Thematic Network (TN) with Fuzzy Cognitive Maps (FCMs) modeling and simulation, and Exploratory Modelling and Analysis (EMA). Furthermore, I developed a thematic proximity measure to determine the weights of the edges in the FCMs based on research texts, thus reducing the need for including subject matter experts in modeling studies.
\end{abstract}

This work makes several contributions: on a theoretical level, it shows the inherent dynamics of HROs and points to several limitations in existing High Reliability Organizations Theory (HROT) as well as uncertainties regarding the efficacy of some 
safety interventions. On a practical level, it provides a tested and verified planning tool for the safety decision-makers that can also serve as the foundation of future safety culture training.

Finally, it makes several contributions to the FCM methodology, namely a model architecture that combines knowledge from the literature with that of human experts, the introduction of thematic proximity coefficient, and the adaptation of model testing strategies from the literature on Systems Dynamics. 


\section{Dedication}

I dedicate this work to my beloved father, Abdulaziz Alibage, who has unexpectedly passed away before witnessing what he has been eagerly awaiting - to see me become who he always wanted me to be.

Father, together with my precious and wonderful mother, Amel Alibage, I proudly declare that this entire work is the product of your genuine and unconditional love, as well as your passion, keenness, affection, care, encouragement, motivation, indefinite support, and continuous prayers. 


\section{Acknowledgments}

Firstly, I would like to express my sincere appreciation and gratitude to my advisor Prof. Antonie Jetter for the endless support of my Ph.D. study and the related research for her patience, motivation, and immense knowledge. Her guidance helped me tremendously in all the time of research and writing of this dissertation. Besides, I am so grateful for being her student, and I am also very thankful for knowing her on a personal level, which helped me, even more, to cope with difficult times and roughnecks in this journey. Without her, this work would not be as it is today.

I also would like to thank my dissertation committee members: Prof. Wayne Wakeland, Prof. Charles Weber, and Prof. Steven Scyphers, for their delightful guidance, insightful comments, continuous support, and encouragement, but also for the tough questions they have used to ask me, which motivated me, even more, to widen my research from various perspectives.

Moreover, I would like to thank all my beloved family members for their sincere and indefinite love, real courage, continuous support, and enthusiasm for reaching this. Being a part of my wonderful family, where love is widely spread, minds are always opened, and education and knowledge are so encouraged and welcomed, have always helped me make my dreams come true.

I also would like to thank my very dear friends, my classmates, and the members of my research group, as well as the brilliant faculty and staff at the Department of Engineering and Technology Management at Portland State University. 
Finally, I would like to thank the National Academies for Engineering, Science, and Medicine for funding my doctoral study throughout two funded projects (grants): Collaborative modeling with fuzzy cognitive maps: A novel approach to achieving safety culture (2016-2019), and Bringing High-Reliability Safety Culture Decisions into Focus: Training with Interactive Fuzzy Cognitive Mapping (2020-2023). Without these funding opportunities, I would not be able to achieve this goal.

Also, I would like to thank the Center for Offshore Safety for their support and assistantship during this work. 


\section{Table of Contents}

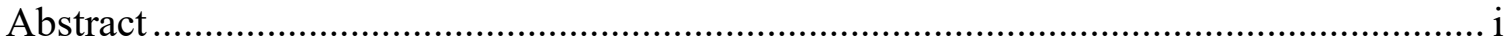

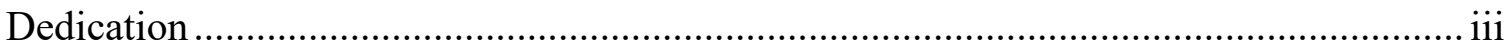

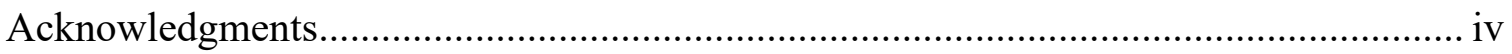

List of Tables .....................................................................................................

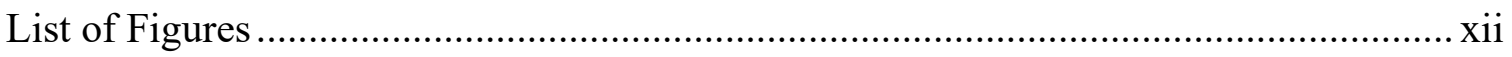

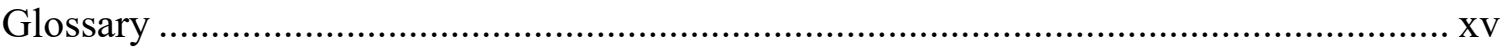

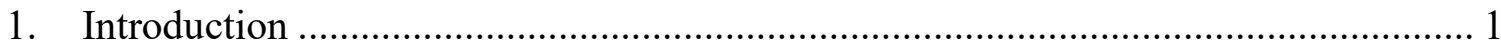

2. High Reliability Organizations Theory (HROT) …........................................... 7

2.1 Safety as a System: High Reliability and Normal Accident Theory ...................

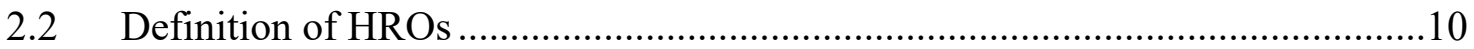

2.3 HROs as Organizational Safety Culture.................................................... 12

2.3.1 Organizational Culture ........................................................................... 12

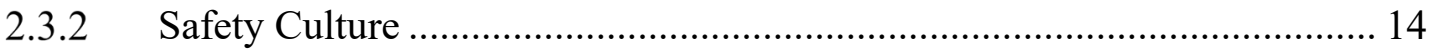

2.3.3 Safety Culture and Safety Management Systems (SMSs) ....................... 15

2.4 HROs Compared to Other Approaches ........................................................... 17

2.5 Research Framework: A System Approach to Achieving HROs ......................22

2.5.1 Earlier Interpretations of HROs' Characteristics ................................... 22

2.5.2 The Specific Characteristics of HROs: A Present-Day Framework ........... 26

2.5.3 The Five Principles of HROT ............................................................... 28

2.5.4 Organizational Reliability (Collective Mindfulness).............................. 36

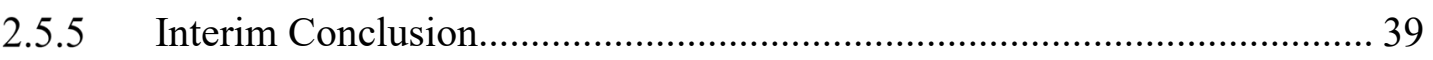

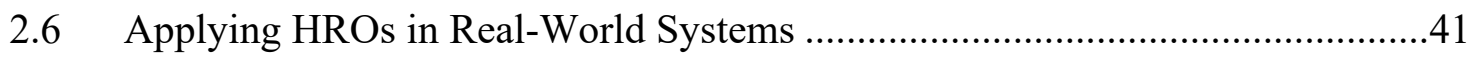

2.6.1 Applicability of HROs in Different Systems and Industries .................... 41 
2.6.2 Assessing HROs in Practice........................................................................ 44

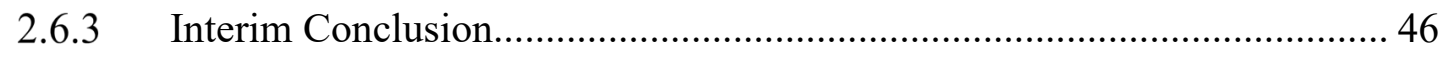

2.7 Research Context: The Offshore Oil and Gas Industry.......................................47

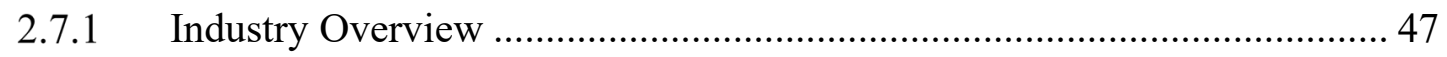

2.7.2 Safety Performance and Safety Culture ....................................................... 48

2.7.3 Challenges to a Strong Safety Culture ………………………………..... 51

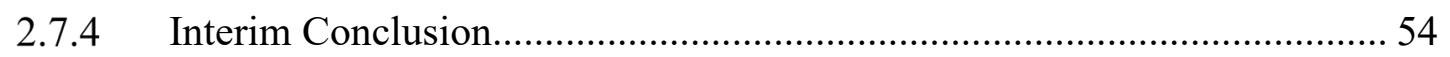

2.8 Conclusion, Research Gaps, Objectives, and Questions ...................................55

2.8.1 Lack of System Perspectives …………………………………………. 56

2.8.2 Lacking Contextualization for Offshore Oil and Gas ................................. 56

2.8.3 Lack of Practical Planning Approaches ...................................................... 57

2.8.4 Summary of Research Gaps, Objectives, and Questions ............................. 57

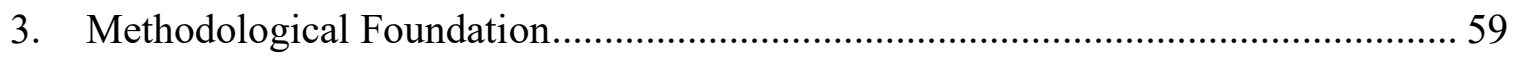

3.1 Methodological Selection..........................................................................60

3.2 Fuzzy Cognitive Maps (FCMs) .....................................................................62

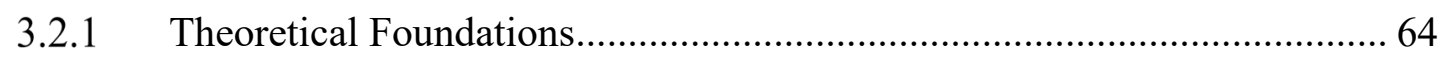

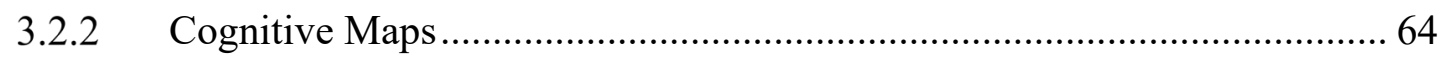

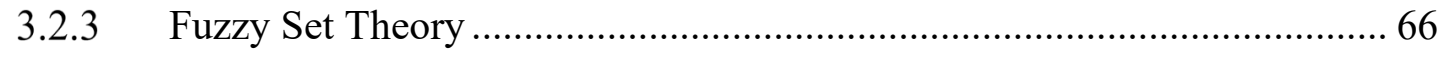

3.2.4 Integrating Artificial Neural Network Theory in FCMs.............................. 69

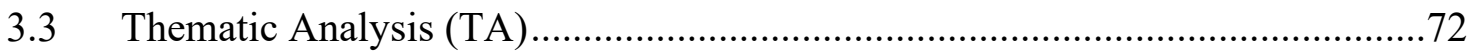

3.3.1 Theme Definition and Development....................................................... 73

3.3.2 Phases of Thematic Analysis .................................................................... 76

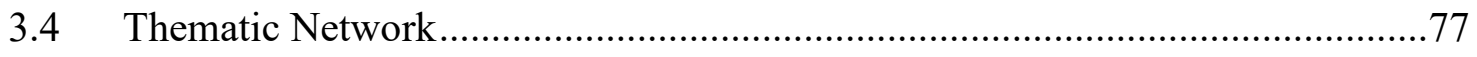

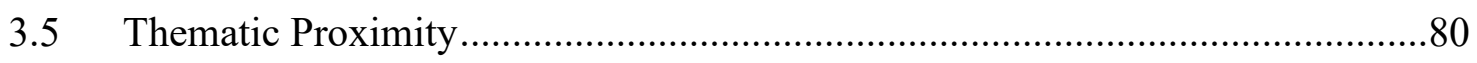


3.6 Exploratory Modelling and Analysis (EMA) .......................................... 85

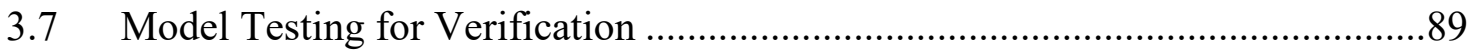

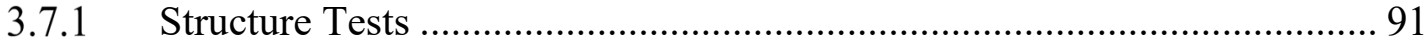

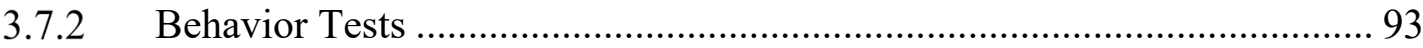

3.8 Model Simulation and Scenario Planning ....................................................95

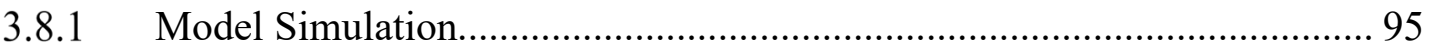

3.8.2 Scenario Planning ............................................................................. 100

4. The Process of Building the Core FCM Model ................................................. 102

4.1 Phase 1: Creating the Collective Cognitive Map (CM) .................................104

4.1.1 Step 1: Selection of Published Research........................................... 104

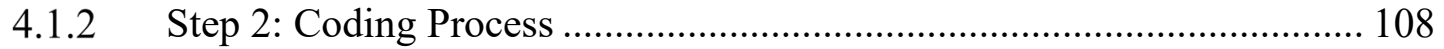

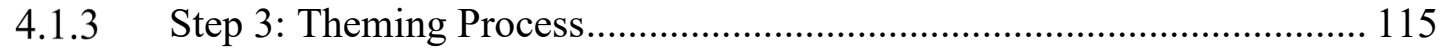

4.2 Phase 2: Converting the Cognitive Map (CM) into (FCM) ............................129

4.2.1 Step 1: Content Filtering and Retrieving .......................................... 129

4.2.2 Step 2: Calculating the Weight of the Edges ...................................... 130

4.2.3 Step 3: Examining the Relevance of t-Coefficient ............................... 133

5. Model Preliminary Application (Pilot Study) ................................................... 138

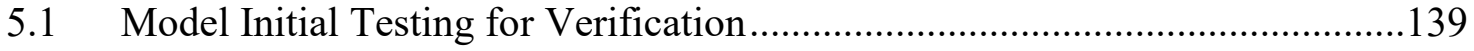

5.1.1 Structure Test: Structure Confirmation Test:................................... 140

5.1.2 Behaviors Tests: Extreme Conditions and Sensitivity Analysis Tests ..... 140

5.2 Model Contextualization Procedure .............................................................144

5.2.1 Selecting Relevant Managerial Interventions ..................................... 147

5.2.2 Applying Interventions to the Core Model ...................................... 151

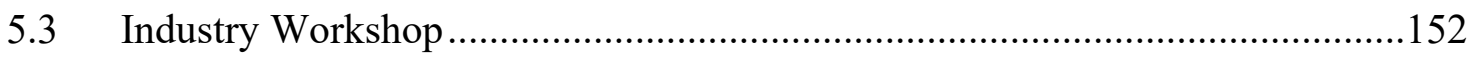


5.3.1 Preparing the Workshop …………………......................................... 152

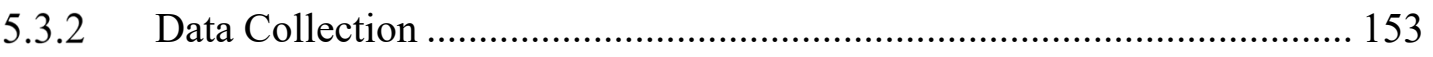

5.3.3 Model Simulation and Behaviors............................................................ 154

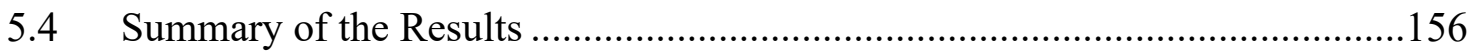

6. Model Application (Expanded Study) ................................................................... 158

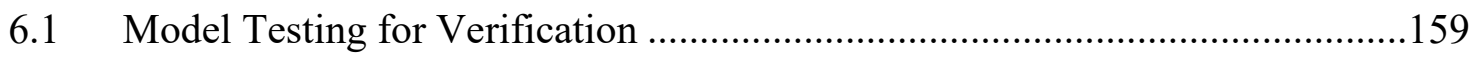

6.1.1 Boundary Adequacy Test.................................................................. 159

6.1.2 Behavior Anomaly Test ........................................................................ 162

6.1.3 Family Member Test......................................................................... 165

6.1.4 Surprise Behavior Test...................................................................... 166

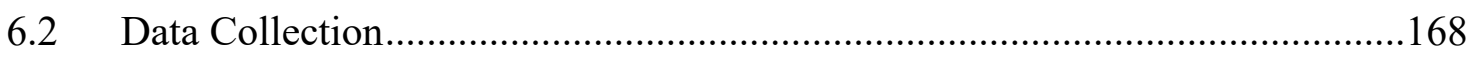

6.2.1 Participants and Recruitment ............................................................... 168

6.2.2 Survey Design and Administration........................................................... 169

6.3 Model Simulation and Behaviors .............................................................171

6.3.1 Traditional FCM Simulation................................................................... 172

6.3.2 Explorative FCM Simulation Using EMA Technique................................ 183

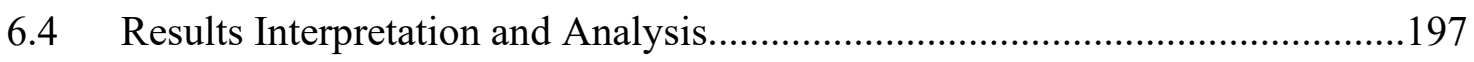

6.4.1 Results of Model Simulation Using Traditional FCM............................... 197

6.4.2 Results of Model Simulation Using Explorative FCM (EMA Method)... 208

7. Summary and Discussion of Results .................................................................... 215

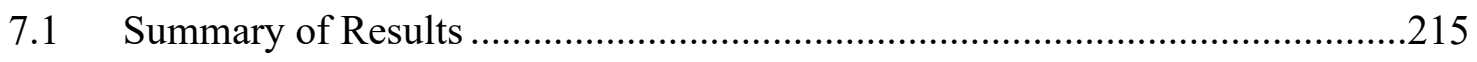

7.1.1 HROT Principles as a System................................................................ 215

7.1.2 Model for Decision Support and Training ............................................. 217

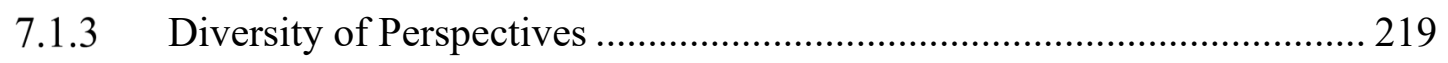


7.1.4 Industry Insights........................................................................... 220

7.2 Review of Research Questions and Results .....................................................222

7.2.1 Addressing the lack of System Perspectives.............................................. 223

7.2.2 Addressing the Lack of Contextualization for Offshore Oil and Gas....... 227

7.2.3 Addressing the Lack of Practical Planning Approaches............................. 228

8. Research Limitations and Contributions ……………....................................... 230

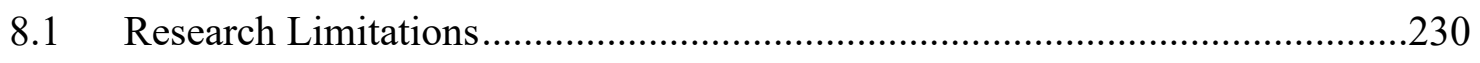

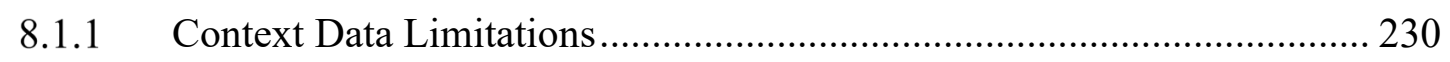

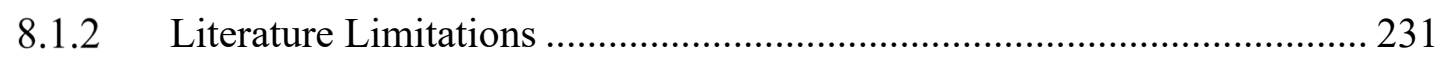

8.1.3 Methodological Limitations................................................................... 232

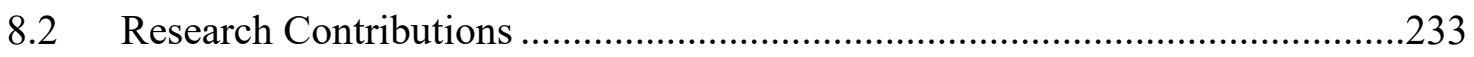

8.2.1 Theoretical Contribution ...................................................................... 233

8.2.2 Methodological Contribution............................................................... 234

8.2.3 Practical Contribution .......................................................................... 236

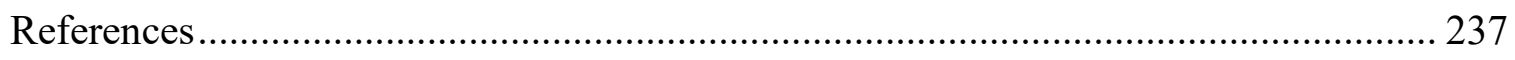

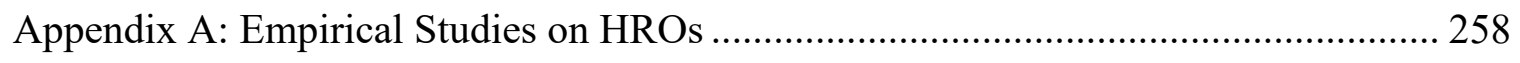

Appendix B: Selected Research Publications .............................................................. 264

Appendix C: Labels and Definitions of the Final Codes ................................................ 267

Appendix D: Codes to Themes Development Schemes …………………………........ 282

Appendix E: Thematic Structures and Networks.......................................................... 287

Appendix F: FCM's Edges Weights Comparison Based Three Measures ..................... 304

Appendix G: Materials of the Contextualization Procedure ................................................ 324

Appendix H: Materials and Outcomes of the Pilot Study.............................................. 327

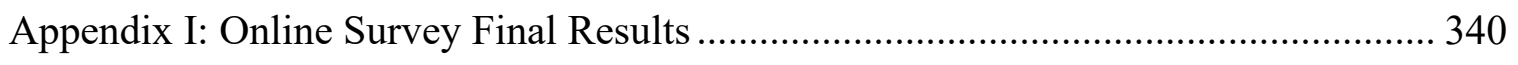




\section{List of Tables}

Table 1: Layers of Culture Based on Different Perspectives....................................... 13

Table 2: Approaches to Safety Culture Compared to HROs ........................................ 20

Table 3: Typology of Organizational Culture That Deals with Safety Information......... 23

Table 4: Research Gaps, Research Objectives, and Research Questions ....................... 58

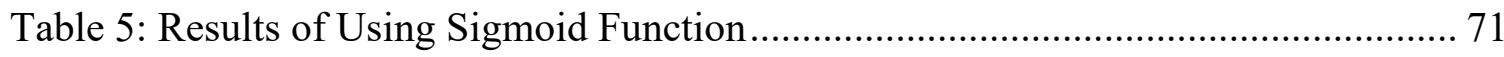

Table 6: Results of Using Hyperbolic Tangent Function ...................................... 72

Table 7: Combinations of Model Simulation Methods and Approaches......................... 98

Table 8: Predefined Criteria for Selecting Publications on HROT.............................. 106

Table 9: Number of Codes and Connections Over the Steps of the Coding Process ..... 114

Table 10: Thematic Structure of Preoccupation with Failure (PWF) ............................ 122

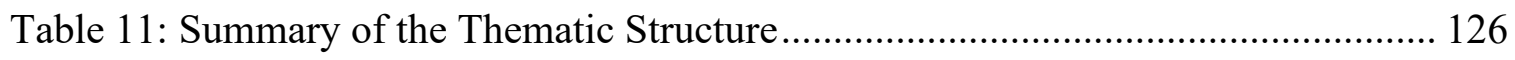

Table 12: Phase1 Final Findings Statistics .............................................................. 127

Table 13: FCM Preferred State and Metrics ......................................................... 131

Table 14: Selected Managerial Interventions .......................................................... 148

Table 15: Example of How Scenarios Based Interventions Established ...................... 151

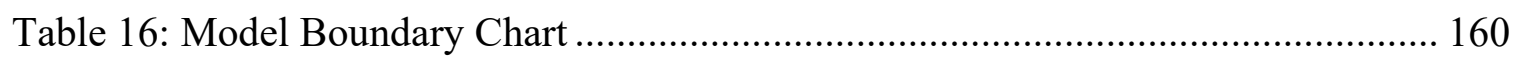

Table 17: Selected Cases for Behavior Anomaly Test ............................................ 163

Table 18: Numerical Outcomes of HROT Principles and Organizational Reliability.... 177

Table 19: Scenario Based on Grouped Interventions ................................................ 180

Table 20: Numerical Outcomes of HROT Principles and Organizational Reliability.... 182

Table 21: Cases Where HROT Principles Found to Be Sensitive to Edges' Weight..... 186 


\section{List of Figures}

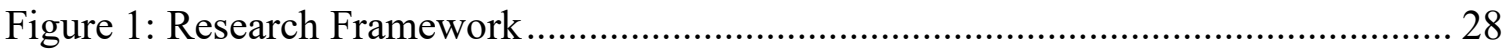

Figure 2: Offshore Incident Statistics 2007-2018 ......................................................... 49

Figure 3: Methodological Foundation .......................................................................... 59

Figure 4: Simple Causal Cognitive Map and the Corresponding Adjacency Matrix ....... 65

Figure 5: Simple FCM and the Corresponding Adjacency Matrix.................................. 67

Figure 6: Signal Processing in an Artificial Network ..................................................... 70

Figure 7: Effects of Sigmoid Function............................................................................ 71

Figure 8: Effects of Hyperbolic Tangent Function ........................................................ 72

Figure 9: Various Paths to Develop Themes from Textual Data....................................... 75

Figure 10: Thematic Network (Global Theme) ……………………............................ 78

Figure 11: Text Vectors Projection in Cosine Similarity Index ...................................... 82

Figure 12: Possible Cases Resulted from The Use of c-Coefficient .................................. 83

Figure 13: Graphical Representation of How the Variable of t-coefficient Identified ..... 84

Figure 14: Graphical Representation of Explorative VS. Traditional FCM Modeling .... 88

Figure 15: The Process of Model Verification ............................................................... 90

Figure 16: The Process of Building the Core FCM Model Based HROT Principles ..... 103

Figure 17: The Process of Selecting Relevant Publications ........................................... 107

Figure 18: The Process of Coding Textual Data.......................................................... 110

Figure 19: Screenshot of Atlas.ti to Show How Documents Were Coded ...................... 112

Figure 20: Illustration Example of the Theming Process ……………........................... 119

Figure 21: Conceptualization of Developing HROT Cognitive Map .............................. 120 
Figure 22: Thematic Network (PWF): Developed with Atlas.ti. 125

Figure 23: Thematic Network of Organizational Reliability: Developed with Atlas.ti.. 128

Figure 24: Example of Retrieving and Filtering Words in Textual Documents............. 130

Figure 25: FCM based HROT Five Principles: Developed with Mentalmodeler.com... 132

Figure 26: Edges' Weights Comparison Based on Three Techniques ......................... 137

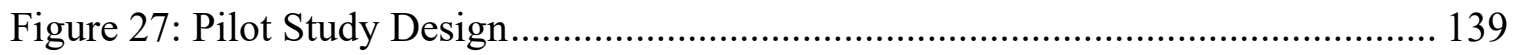

Figure 28: Sensitivity Analysis Test Before and After Modifying the Model Structure 142

Figure 29: Examples of Model Testing for Verifications (Uncertainty Analysis) ......... 143

Figure 30: Graphical Representation of the Process of Model Contextualization.......... 146

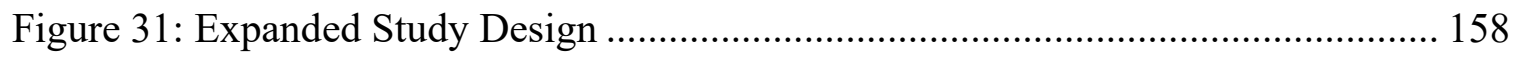

Figure 32: Changes in the Five HROT Principles Based on Behavior Anomaly Test... 164

Figure 33: Simulation Results of the Family Member Test........................................ 166

Figure 34: Model Behavior Based on Literature VS. Experts' Opinions ...................... 167

Figure 35: Screen Shot of the Expanded Study Online Survey with Qualtrics ............. 170

Figure 36: Model Simulation Based on Individual Interventions (1) .......................... 175

Figure 37: Model Simulation Based on Individual Interventions (2) .......................... 176

Figure 38: Comparison of Model Simulation Based on Different Activation Levels .... 178

Figure 39: Model Simulation Based on Grouped Interventions ................................. 181

Figure 40: Sensitivity Analysis Based on Edges’ Weight Variations........................... 185

Figure 41: EMA Outcomes Based on 1000 and 5000 Iterations .............................. 188

Figure 42: EMA Outcomes Based on 10000 and 50000 Iterations ............................ 189

Figure 43: EMA Outcomes Based on 100000 and 150000 Iterations ............................ 190 
Figure 44: Effects of Individual Interventions on HROT Principles Based EMA (1)... 192

Figure 45: Effects of Individual Interventions on HROT Principles Based EMA (2)... 193

Figure 46: Effects of Individual Interventions on HROT Principles Based EMA (3)... 194

Figure 47: EMA Simulation Based on Grouped Interventions................................... 196 


\section{Glossary}

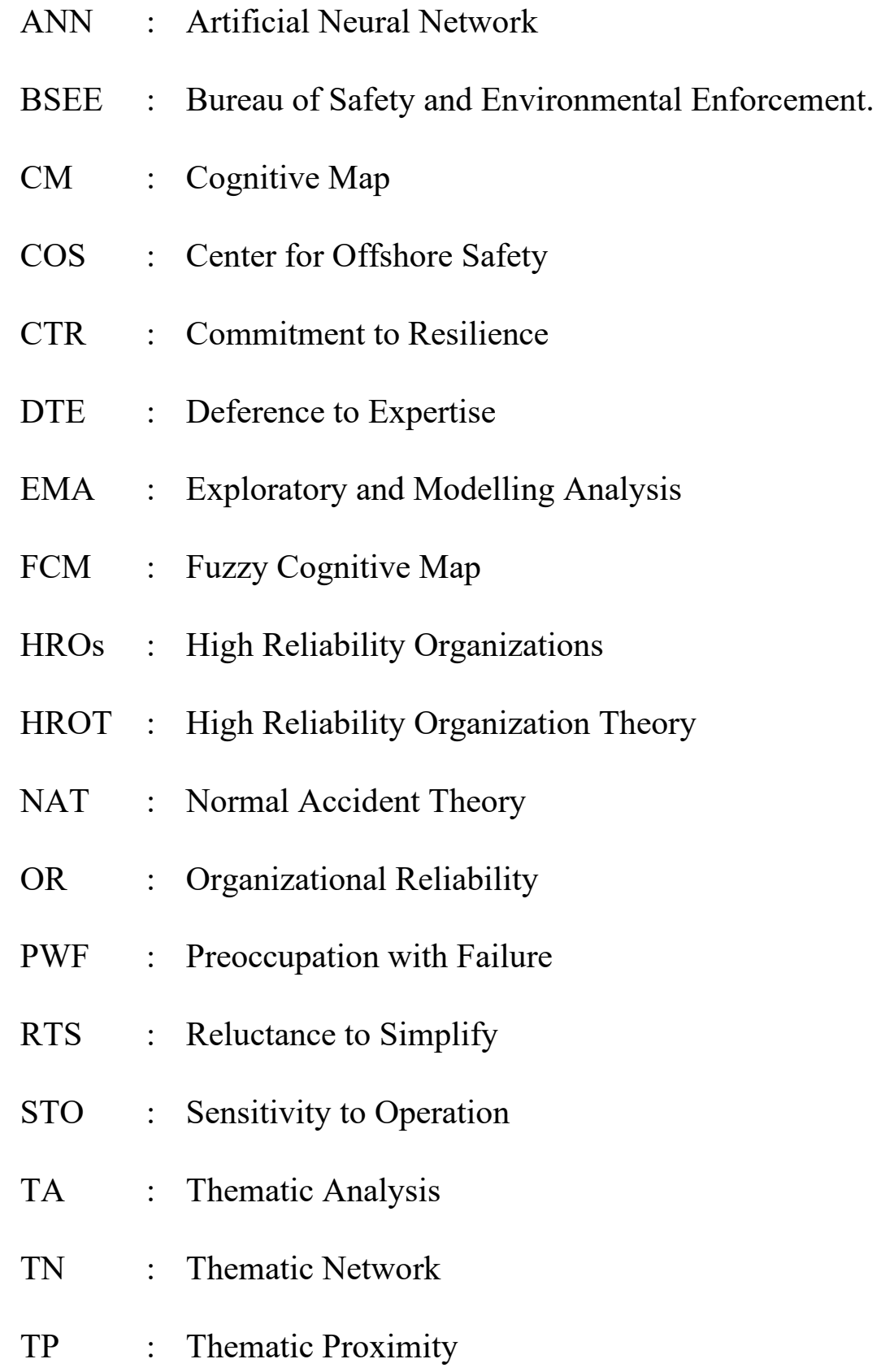




\section{Introduction}

Large-scale accidents in socio-technical systems can have devastating consequences, including loss of lives, poor health outcomes, damaged ecosystems, reduced economic opportunities, disruption of local communities, and negative impacts on government budgets. A case in point is the 2010 Deepwater Horizon explosion and oil spill, which resulted in 11 fatalities, 17 severe injuries (BSEE, 2014), massive environmental damage (National Commission, 2011), and reduction in BP's bottom line and taxable income by almost $65^{1}$ billion US dollars. These consequences should be a compelling incentive for organizations to prevent such events. However, the rate of accidents in the offshore oil and gas remain consistently and stubbornly high (BSEE, 2015; 2016; 2017; National Academies, 2016), which results in the need to move beyond the study and analysis of accidents causations and aftermaths: besides, effective strategies and reliable procedures for prevention and mitigation of accidents must be developed and implemented, such as error anticipation, detection, reporting, and timely correction.

High-profile accidents (also known as organizational accidents) are rare events that happen to systems or subsystems due to multiple organizational factors that can be identified in almost every organizational disaster (Reason, 1998; Pidgeon, 2010) and that are critical to preventing them (Leveson et al., 2009; Reason, 2000). These factors include but not limited

1 BP Deepwater Horizon costs balloon to $\$ 65$ billion: https://www.reuters.com/article/us-bpdeepwaterhorizon/bp-deepwater-horizon-costs-balloon-to-65-billion-idUSKBN1F50NL 
to system complexity ${ }^{2}$ and elements coupling ${ }^{3}$ (Perrow, 1984), sophisticated types of machinery (Priest, 2008), conflicting goals, performance pressure (Weick et al., 1999), deadlines, intensifying commitment, a decline of resource slack (Vaughan, 1996; Reason, 1998), and reward systems that promote productivity and undermine safety (Weick \& Sutcliffe, 2015).

Within the literature on organizational accidents, a significant research stream recommends adopting the practices of so-called High Reliability Organizations (HROs) to overcome these factors and prevent and control errors that lead to such accidents (Amalberti, 2013; Vogus \& Sutcliffe, 2007). HROs practice is the outcome of High Reliability Organizations Theory (HROT): A philosophy of system-resilience to manage risks in complex sociotechnical systems (Roberts \& Rousseau, 1989; Roberts, 1990), which leads organizations that operate reliably. This theory was developed by researching so-called HROs, which are defined as "a subset of hazardous organizations that have enjoyed a record of high safety over long periods of time" (Sutcliffe, 2011, p. 134), such as nuclear power plants, air traffic control (Vogus et al., 2010; Weick et al., 1999), and naval aircraft carriers (Roberts, 1990a).

HROs practice results in a company culture that strongly endorses learning and fosters work environments where errors, awareness, and concerns can be openly discussed without fear of blame or accusation (Rochlin, 1996; Weick et al., 1999). It builds and continuously updates repertoires for organizational knowledge to support developing a resilient system

\footnotetext{
2 System complexity denotes the extent whereby the interactions among the system elements (e.g., people, hardware, processes, etc.) are unpredictable and invisible.

3 Coupling refers to the degree of interdependence among the system elements.
} 
that enables people to anticipate, contain, and cope with unexpected events and learn from these events and quickly return to normal conditions (Sutcliffe, 2011; Weick \& Sutcliffe, 2015). This approach consists of five principles: Preoccupation with failure, reluctance to simplify, sensitivity to operations, commitment to resilience, and deference to expertise. An extensive discussion on HROT, HROs, and the five principles will be provided in Chapter 2 of this dissertation.

Although the literature on HROs builds on more than thirty years of academic research and provides an extensive discussion and description of the five principles, it fails to identify or provide methods for implementing HROs practices in different organizational contexts (Hales \& Chakravorty, 2016; Roberts \& Martelli, 2016). Moreover, the literature lacks a clear explanation of how these five principles are correlated and how they can result in a capability for both discovering and managing unexpected events (Hopkins, 2007a; Lekka, 2011). As a result, there is an essential need to identify and model all factors that boost as well as factors that impede the development of organization reliability. Such a model ensures that organizations can be assessed not only for their aspiration to become HROs, but also for their actual capacity, efforts, and the level of their success in the process.

To solve the presented problems, the overall objective of this dissertation is to develop a novel approach by using Fuzzy Cognitive Maps (FCM) to build a theory-driven model of HROs that is grounded in the literature on HROT. FCMs are created with a system thinking technique that explores the dynamic behavior of causal maps through fuzzy set theory and neural networks (Kosko, 1986; 1988). In this work, I combine FCM modeling with Exploratory Modeling and Analysis (EMA), which is a simulation technique that deals 
with uncertainties in complex modeling (Kwakkel \& Pruyt, 2013). EMA copes with modeling so-called "deep" uncertainties about system states and system structures by considering not only a single model but multiple models with different structures. Each of the models is simulated for various inputs resulting in a range of different outcomes; therefore, the decision-makers can thoroughly explore the uncertainty of their decisions.

The approach of this dissertation and the resulting model of HROT are intended to be generalizable for all HROs. To evaluate the model's feasibility, usability, and effectiveness, I applied it in the context of the offshore oil and gas industry, which has inherently hazardous operations, generally high accident rates, and a historical record of high-profile disasters.

The objective of developing and evaluating a theory-driven HROT model through the integration of FCM and EMA required answering the following research questions:

1. How can research publications on HROT be analyzed to identify and represent relevant concepts and causal relationships?

2. How can the weight of causal relationships be inferred from qualitative data in research publications?

3. How can information about concepts, causal relationships, and relationships' weights be used to develop a general FCM model of HROT?

4. How can uncertainty about the appropriate model structure be managed in the modeling process?

5. How can the general FCM model of HROT practices be contextualized to the specific settings of offshore oil and gas operations? 
6. How can the contextualized FCM model of HROT practices be used to support safety decision-makers in the offshore oil and gas industry?

In addition to several theoretical, methodological, and practical contributions, answering the above six questions resulted in an innovative approach that, for the first time, assigned weights for the edges of the FCM model by using the same qualitative context used to build it. The model is expected to leverage the existing capabilities of the selected research context in fostering and sustaining a culture of safety that empowers people to cope with unexpected events and mitigate their often-devastating consequences. Moreover, it informs safety decision-makers and guides their prioritization of safety-relevant procedures, and helps them understand how to best achieve a high safety level.

Chapter 2 of this dissertation provides extensive discussions on the-state-of-the-art of high reliability organization theory HROT, as well as an overview of why practicing HROs can lead to achieving safer operations when other available approaches fall short. This chapter also presents the research framework and extensively reviews the offshore oil and gas industry as the selected context of this research. At the end of this chapter, I highlight the identified research gaps.

In Chapter 3, I discuss the methodological foundation for this research, including Fuzzy Cognitive Maps (FCMs), Thematic Analysis (TA), Thematic Network (TN), Thematic Proximity (TP), Exploratory Modelling and Analysis (EMA), methods for model testing for verification, and model simulation and scenario planning. Chapter 4 discusses the process of developing the modeling approach and the actual procedure of building the core FCM model, which I conducted across two research phases. 
In the first phase, I extracted the knowledge about the causes and effects of HROs practices from the literature, using a qualitative approach that combined the method of thematic analysis (Braun \& Clarke, 2006) and the technique of thematic networks (Attride-Stirling, 2001). This phase resulted in the research's cognitive map. In the second phase, I used the thematic proximity method (Armborst, 2017) to quantify the causal relationships between each two causally related concepts in the research's cognitive map, thus converting it to an FCM model.

Chapter 5 covers the process of conducting the third phase of this dissertation, which includes testing the developed FCM model using several verification tests, and then preparing it for the pilot study, which I have undertaken through an industry workshop. This pilot study has evaluated the feasibility and usefulness of the overall research approach based on the opinions of experts from the offshore oil and gas.

Chapter 6 of this dissertation includes the fourth research phase, through which I first subjected the FCM model to another series of model verification tests; then, I expanded the research through an online industry survey. In this phase, I combined EMA with FCM to cope with uncertainties resulting from the complex nature and limited research on HROs in the offshore oil and gas as well as the variations in the opinions of the survey's participants. This chapter also covers the interpretation and analysis of the model simulation results.

Chapter 7 provides a summary and discussion of my research results, while Chapter 8 covers in detail the research limitations and contributions. 


\section{High Reliability Organizations Theory (HROT)}

This dissertation builds on High Reliability Organizations Theory HROT, which explains the safety and reliability of complex technical operations from social and technological perspectives of systems. In particular, the theory emphasizes the importance of organizational practices and mindfulness, which results in a unique organizational culture that leads to improved safety. Accordingly, the following sections will discuss system perspectives on safety, define HROs, introduce the concepts of organizational culture, safety culture, and safety management systems and discuss the unique cultural aspects that allow HROs to maintain high levels of safety and reliability under challenging conditions. The chapter concludes with a framework that explains how the five HROT principles lead to developing a joint capability for organizational reliability and how this research addresses the gaps and plans to close them.

The chapter also includes detailed discussions on the current safety performance and culture of the offshore oil and gas industry as a research context. By the end of this chapter, a summary of the state-of-the-art of HROT, the research gaps, the research objectives, and the research questions are presented.

\subsection{Safety as a System: High Reliability and Normal Accident Theory}

Approaches to improving safety have varied significantly over history (Antonsen, 2009) but were primarily focused on the notion that accidents are either unavoidable events of fate or caused by a single cause, such as a person's ill will or flaws. A more systemic perspective on accidents only emerged relatively recently (Amalberti, 2013) and emphasizes that accidents and disasters are the results of complex factors that contribute to 
things going wrong and complex mechanisms that determine the effects of whatever goes wrong (Hollnage, 2014; Weick \& Sutcliffe, 2015). Among others, Barry Turner's 1978 book Man-Made Disasters shifted the focus from disaster management to disaster prevention (Turner, 1978), and several disciplines began to analyze the factors that lead up to disasters (Hale \& Hovden, 1998). In the 1980s, the growing understanding of humangenerated disasters created a broad debate between two principal schools of thought (Sagan, 1995): System-vulnerability based on Normal Accident Theory (NAT) and system-resilience based on High Reliability Organizations Theory (HROT).

NAT is based on the work of Charles Perrow (1984), who studied the Three Mile Island disaster (i.e., a partial meltdown of a nuclear reactor, which occurred in Pennsylvania in 1979) from a system design and organizational perspective. Perrow (1984) stresses that accidents are inevitable in systems that are characterized by high-complexities and tightcoupling because their design makes large parts of the system invisible to operators and managers so that no individual can fully comprehend latent issues that can lead to accidents when they begin to evolve (Perrow, 1984). As a result, organizational learning is inherently hampered, making systems vulnerable to accidents in their normal course of operations (Perrow, 1984). This problem is getting worse as systems are becoming more complex. Shrivastava (1987), for example, points out that half of the 28 industrial disasters during 1900-1986 have occurred since 1977, and three of them occurred in 1984. NAT explains this as a result of how technical systems are designed and increasingly connected to other systems and states that high-profile disasters will continue to happen. Approaches to reducing risks, such as creating redundancy in complex systems, are considered ineffective 
in preventing accidents and may add additional complexity that causes even higher risk (Perrow, 1984). The NAT thus takes a decisively pessimistic view.

In contrast, HROT theorists (Weick \& Sutcliffe, 2001) urge that system complexity does not necessarily lead to accidents and disasters but can be comprehensible and manageable. This is possible when people pay attention to sustaining performance instead of solely focusing on increasing efficiency and engage in organizational practices that facilitate system comprehension rather than impede it (Weick \& Sutcliffe, 2015). According to Weick (1987), accidents can be reduced in a complex system, even in trying environments, if the system's technical complexity is matched with sufficient levels of human complexity (i.e., diversity of perspective), which he characterizes as "requisite variety." This dissertation takes the same optimistic view and focuses on organizational practices in HROs for improving safety outcomes.

The theory at the center of this investigation, HROT, is in many ways a direct response to the growing number of high-profile disasters that occurred, as NAT had anticipated: society became unsatisfied with just studying and investigating the causation and aftermath of extremely costly accidents (Roberts and Rousseau, 1989) that were, in hindsight, often recognized to be triggered by poor management strategies (Roberts, 1990) and unsafe cultural rigidities (Turner, 1978). This resulted in increasing pressure to devote more attention to operating organizations safely (Roberts, 1990b) by learning from organizations with a positive track record, rather than learning from accidents.

Several high-hazard organizations served as examples, namely the Federal Aviation Air Traffic Control System, the United States Navy, and the Pacific Gas \& Electric Company. 
They had operated nearly error-free for long periods of time (Roberts \& Rousseau, 1989; Roberts, 1990; Rochlin, 1993). Their high operational reliability provided a research opportunity for understanding what leads to safety, rather than accidents.

Starting in 1984, Todd R. LaPorte, Karlene Roberts, and Gene I. Rochlin from the University of California, Berkeley set out to examine the organizational patterns and behaviors associated with these organizations' exceptional performance. The researchers used a multi-method approach, including direct observations, workshops, interviews, and surveys. In 1988, they reported the first project on what they called "High Reliability Organizations" (HROs). The report highlighted the structural patterns, approaches to managing interdependence, decision dynamics, and organizational culture that led these organizations to perform reliably despite the sophisticated technologies they operate and the trying conditions they had been coping with regularly (LaPorte et al., 1988). Together with subsequent research, it provided the theory on high reliability organizations.

\subsection{Definition of HROs}

High Reliability Organizations (HROs) are high-hazard organizations that operate with nearly-accidents-free operations and include nuclear power plants, air traffic control operations (Vogus \& Sutcliffe, 2007a; Weick et al., 1999), and naval aircraft carriers (Roberts, 1990). In HROs, operations take place in environments that are socially and politically challenging (Weick et al., 1999), technologically sophisticated (Weick et al., 1999; Vogus \& Sutcliffe, 2007a), interdependent and timely pressured (Vogus \& Sutcliffe, 2007a), uncertain, and hazardous (Roberts \& Rousseau, 1989). Still, they perform with a constant high safety level nearly error-free (Roberts, 1990). 
The early research on HROs defines them based on their ability to achieve and sustain exceptional performance over a long period of time (Roberts, 1990; Roberts \& Rousseau, 1989). However, the more widely used definition in research on the topic goes beyond these performance characteristics. It includes the objectives or strategic mindset of HROs, including their readiness, commitment, and capacity to perform reliability. It defines HROs as "..organizations that exhibit a strong sense of mission and operational goals stressing not only the objectives of providing ready capacity for production and service but an equal commitment to reliability in operations, and a readiness to assure investment in reliability enhancing technology, processes and personnel resources" (La Porte, 1996, p. 63).

HROs are known for their ability to maintain reliability, as the name and the definition above highlights. In the context of HROs, reliability refers to the performance of the whole system and reflects a culture that embodies doing the right thing at the right time using the proper means even in the face of unexpected events, particularly when no one is watching (Roberts et al., 1994). ${ }^{4}$ A reliable organization remains consistent in its ability to fulfill its purpose, even when a part of its system fails. Reliability ${ }^{5}$ thus is the most critical

\footnotetext{
${ }^{4}$ The term "reliability" in the context of HROs sometimes leads to confusion. Reliability is commonly known as a statistical measure that determines how efficiently and consistently a product, service, or system component(s) functions over a given period. The lower the probability of failure, the higher the reliability. According to LaPorte et al. (1988), reliability is often concerned with production rather than the whole system because the classical understanding of reliability stems from an engineering perspective that reflects "repeatability" or "reproducibility" of actions or patterns of activity (Weick et al., 1999). Over time, the concept became a reflection of work routine, and thus, failed to deal with fluctuating and unexpected working conditions (Weick, et al., 1999). In the context of HROs, reliability refers to the ability of the entire system to deliver, even in the face of unexpected and difficult to control events.

${ }^{5}$ LaPorte et al. (1988) conclude four types of reliability that aspiring organizations must achieve to be HROs. Each has distinguished operating, and managerial implications (LaPorte et al., 1988, p. 7):

- Reliability of aggregate supply: Assures an unbroken flow of production input (materials).

- Reliability of physical infrastructure: Searches for perfection in operation integrity.
} 
competency to defend the organization, its people, and the society against any catastrophic threats and possible damages. It is defined as; "a condition in which the organization demonstrates continuously the capacity to provide expected levels and quantity of services, without off-setting failures of critical processes" (LaPorte et al., 1988, p. 6). As widely acknowledged, the phrase "high reliability" in HROs reflects the idea that high risk and high effectiveness can coexist (Weick et al., 1999).

\subsection{HROs as Organizational Safety Culture}

HROs are characterized by a unique "safety culture," which is commonly understood to encompass the attitudes, beliefs, perceptions, and values that members of an organization share concerning safety (Cox \& Cox, 1991). However, understanding the construct of safety culture requires having a working understanding of the concept of organizational culture (Guldenmund, 2000; Glendon \& Stanton, 2000; Götvall, 2014). Accordingly, this section first describes the organizational culture in general, followed by a discussion of the concept of safety culture and the unique features of the culture in HROs. Because many companies aim to track their progress towards improving safety culture through the socalled safety management system, the section will discuss these systems.

\subsubsection{Organizational Culture}

Organizational culture is the set of values, anticipations, practices, and shared vision that guide and inform all the organization members' behaviors. One of the commonly

- Reliability of signals: Searches for perfect accuracy and timeliness of communication signals.

- Reliability of human response (operational reliability): Seeks to perfect performance of human operators. 
referenced conceptualizations of organizational culture is provided by Schein (2010), who developed a layered model of organizational culture. It consists of a core layer (underlying assumptions, e.g., human nature), a middle layer (espoused beliefs and values, e.g., strategies, goals, philosophies), and an outer layer (artifacts, e.g., greeting rituals, dress). The visible outer layer points to the invisible other layers but requires considerable interpretation. It is relatively easy to change, but this change can occur without affecting the "deeper" inner layers of culture.

Other pioneering authors agreed with the notion of organizational culture as "layered," yet they disagreed concerning the layers' constructs, sequences, and interpretations (Guldenmund, 2010). Table 1 below lists different but frequently cited and used models of culture.

Table 1: Layers of Culture Based on Different Perspectives: Adopted from Guldenmund (2010)

\begin{tabular}{|c|c|c|c|c|}
\hline Central Core & Layer 1 & Layer 2 & Layer 3 & Author(s) \\
\hline $\begin{array}{c}\text { Basic } \\
\text { Assumptions }\end{array}$ & Espoused values & Artifacts & Non & (Schein, 2004; 2010) \\
\hline Values & Rituals & Heroes & Symbols & (Hofstede, 1986) \\
\hline Values & Heroes & Rites and Rituals & $\begin{array}{c}\text { Communication } \\
\text { Network }\end{array}$ & (Deal \& Kennedy, 1982) \\
\hline $\begin{array}{c}\text { Bassic } \\
\text { And Values }\end{array}$ & $\begin{array}{c}\text { Beliefs, } \\
\text { Attitudes } \\
\text { and Conventions }\end{array}$ & $\begin{array}{c}\text { Systems and } \\
\text { Institutions }\end{array}$ & $\begin{array}{c}\text { Artifacts and } \\
\text { Products; Rituals } \\
\text { and behavior }\end{array}$ & (Spencer-Oatey, 2000) \\
\hline
\end{tabular}

Cultures exist on all levels of an organization, and there are differences between the cultures of organizational units (e.g., "engineering" culture vs. "marketing" culture, "team culture"). However, when members of an organization share history, occupational backgrounds, and technology requirements, some aspects of culture can be found across 
all organizational levels ("company culture"), and even across different organizations in the industry (IAEA, 2002). For example, the offshore oil and gas industry culture is often described as; "male-oriented, macho, rough and tough, and can do" (National Academies, 2016, p. 20).

\subsubsection{Safety Culture}

Within the broad concept of organizational culture, many authors focus on safety culture. The term is defined as "a specific set of beliefs, norms, attitudes, roles, and social and technical practices that are concerned with minimizing the exposure of employees, managers, customers and members of the public to conditions considered dangerous or injurious" (Turner et al., 1989, p. 4). The first use of the term "safety culture" is usually attributed to the summary investigation report of the International Nuclear Safety Advisory

Group (INSAG) concerning the Chernobyl disaster in 1986 (INSAG, 1991). This report examined the factors behind this catastrophic event as an indication of a weak or lacking safety culture; "The accident can be said to have flowed from deficient safety culture" (Edwards et al., 2013, p. 23). Since then, the term has been progressively used in research that aims to define and identify factors that strengthen safety (Vu \& De Cieri, 2014), especially in the nuclear industry (INSAG, 1991).

The relationship between organizational culture and safety culture is a matter of considerable debate. Several scholars, such as Parker et al. (2006); Clarke (1999); Aboagye-Nimo et al. (2013); Götvall, (2014), and others consider safety culture as a subset of organizational culture (i.e., the meaning of the beliefs, values, and behaviors as they relate to health and safety). Other authors, such as Antonsen (2009), point out that 
understanding safety culture as a subordinate to organizational culture creates confusion because it would require an understanding of which part of the organizational culture influence safety or not and because it reduces culture to a rigid property with stable characteristics (Antonsen, 2009). In contrast, Reason (1997), Hopkins (2006), and Cooper (1998) emphasize that safety culture must be treated independently to achieve focus on and dedication to safety. Specifically for the field of engineering and management, Silbey (2009) urges that safety culture encompasses the commonly shared and constant set of practices by which all members of the organization learn the lessons from mistakes to minimize risk and maximize safety while achieving production goals.

This dissertation is based on HROT, and according to theorists such as Weick (1987), Klein et al. (1995), and Weick \& Sutcliffe (2001), safety culture is inseparable from organizational culture. The similarities in definition and perception between the two concepts are rooted in the notion that safety culture is, in fact, an organizational culture that underlines safety. Weick (1987) suggests that organizational culture is the source of reliability in all HROs, while scholars such as Klein et al. (1995) have empirically shown that HROs share cultural characteristics related to the risk and dangers of their technologies and, as a result, likely also share organizational culture.

\subsubsection{Safety Culture and Safety Management Systems (SMSs)}

A Safety Management System (SMS) is defined as "a systematic approach to managing safety, including the necessary organizational structures, accountabilities, policies and procedures" (ICAO, 2013, p. xii). SMSs began to emerge in the 1990s, when the realization that significant accidents are organizational, systemic problems, rather than single events, 
shifted the perspective of safety research. Researchers and practitioners began to apply general management principles such as setting targets and tracking progress towards targets, to the specific field of safety (Antonsen, 2009) and created integrated systems for managing safety (Richter, 2003).

Early applications of SMSs created a debate regarding their effectiveness in reducing risks in complex systems. On the one hand, SMSs improved safety performance measured by traditional safety indicators ${ }^{6}$ (i.e., FAR, LTIF, and TRCF) ${ }^{7}$ (National Academies, 2016). However, after these initial improvements, the indicators reached a plateau (Hudson, 2007; Götvall, 2014; Fleming, 2001; Carnino, 2000), and none reached zero (Hudson, 2007). This meant that there was evidence that safety management interventions had positive impacts on employees' health and safety but could not produce consistently high levels of safety or "zero accidents." Thus, results were inconclusive, and safety researchers were unsure if they should recommend or discourage SMSs (Robson et al., 2007).

The investigation of SMSs in the context of specific accidents that had occurred despite the implementation of SMSs, could not resolve the debate, either (Hopkins, 2007b; National Academies, 2016). For example, Hopkins (2007b) concluded after studying the 1996 Gretley Coal Mine disaster that SMSs could not ensure safety in complex systems. However, other authors countered that perhaps the complex paper-based management

\footnotetext{
${ }^{6}$ Safety indicators are defined AS "a data-based safety parameter used for monitoring and assessing safety performance" (ICAO, 2013, p. xii).

${ }^{7}$ FAR: fatal accident rate, LTIF: lost time injury frequency, and TRCF: total recorded case frequency.
} 
system that SMSs were built on, not the safety management approach per se, caused the failure (Borys et al., 2009).

Academic scholars, governments, and practitioners began to increasingly point to "safety culture" as the missing ingredient in SMSs, stating that an effective SMS can never be achieved with the absence of strong safety culture (Piers et al., 2009; National Academies, 2016). Today, there is a broad consensus that SMSs by themselves do not create safety and that cultural approaches are needed that go beyond the enactment of stricter rules, regulations, and unfeasible supervision (Cooper, 2013; National Academies, 2016). Because culture influences people's perceptions (i.e., what people see, feel, think, hear, say, etc.), it affects people's decisions and behaviors in an organization, which eventually drives the outcomes and the performance of safety (National Energy Board, 2014). Similarly, Weick \& Sutcliffe (2015) observe that if the espoused values (i.e., what is essential, right, useful, and desirable) are treated as core beliefs, those beliefs serve as work rules. Still, they do so with more flexibility than traditional practices, regulations, and process descriptions. This permits cultural approaches to eventually reach all the subdivisions of the organization (Parker et al., 2006). Accordingly, this dissertation focuses on cultural approaches to safety, not SMSs. These cultural approaches can exist in parallel to SMSs and be fostered by well-designed SMSs.

\subsection{HROs Compared to Other Approaches}

The research on HROT emerged when academia, governments, and the public became increasingly interested in the systemic nature of safety and the importance of culture. The research, therefore, shares several characteristics with other frameworks and approaches 
for safety, which were created with the intention to break down the multifaceted concept of safety into several and distinct dimensions (also labeled as attributes, characteristics, elements, or traits) to understand, enact, and assess safety culture. Table 2 lists seven selected approaches, including HROs practice, for the purpose of comparison.

Although all these approaches were developed based on technical, social, and organizational perspectives (National Academies, 2016), their attributes vary in the number, labels, and descriptions. Compared to HROs, some of the other six approaches in Table 2 consist of more dimensions, which might be mistaken to cover broader conceptions of positive safety culture. In particular, the HROs approach does not provide dimensions for leadership and communication. However, it does not ignore its role but acknowledges the influence of leadership and communications patterns in every practice. As the previous discussion shows, HROT provides a broad and in-depth discussion and interpretation of strong foundations' safety culture attributes. The principles of HROT were theorized based on empirical and observational studies in the specific context of high-hazard and high reliability organizations in different industries (National Academies, 2016; Amalberti, 2013; Grabowski \& Roberts, 2016).

In contrast, some of the other frameworks are more industry-focused. For example, the safety culture policy statement of the Bureau of Safety and Environmental Enforcement (BSEE, 2013) consists of nine cultural dimensions. Yet, these dimensions are not commonly recognized in the industry (i.e., offshore oil and gas) that they were developed for (National Academies, 2016). The dimensions also lack the discussions on developing individuals' competencies, which other frameworks emphasize, particularly HROs 
(National Academies, 2016). Moreover, the BSEE and other policy statements, such as the safety culture policy statement by the U.S. Nuclear Regulatory Commission, do not provide explanations on how to interpret, interrelate, and implement framework dimensions within specific contexts and how to adapt them to other contexts and industrial environments, which reduces their practice relevant. Even practice-focused guidance, such as BSEE's requirement to provide scales for assessing workers' safety performance, does not, according to Paranhos et al. (2017), provide the information needed to achieve the characteristics of a highly reliable organization that is resilient in the face of unexpected events. 
Table 2: Approaches to Safety Culture Compared to HROs: Adopted from (National Academies, 2016)

\begin{tabular}{|c|c|c|c|c|c|c|c|}
\hline Approach & $\begin{array}{c}\text { (BSEE, 2013) } \\
\text { Bureau of } \\
\text { Safety and } \\
\text { Environmental } \\
\text { Enforcement }\end{array}$ & $\begin{array}{l}\text { (U.S.NRC, } \\
\text { 2011) } \\
\text { U.S. Nuclear } \\
\text { Regulatory } \\
\text { Commission }\end{array}$ & $\begin{array}{l}\text { (Christian et al., } \\
\text { 2009) }\end{array}$ & $\begin{array}{c}\text { (Cox \& Cheyne, } \\
\text { 2000) }\end{array}$ & $\begin{array}{l}\text { (The Health } \\
\text { Foundation, } \\
\text { 2011) }\end{array}$ & (Reason, 1997) & $\begin{array}{c}\text { Research on } \\
\text { HROs } \\
\text { (Weick et al., } \\
\text { 1999; Weick \& } \\
\text { Sutcliffe, 2015) }\end{array}$ \\
\hline Leadership & $\begin{array}{l}\text { Leadership is } \\
\text { committed to } \\
\text { safety values } \\
\text { and actions. }\end{array}$ & $\begin{array}{l}\text { Leadership is } \\
\text { committed to } \\
\text { safety; safety } \\
\text { has priority over } \\
\text { other demands. }\end{array}$ & $\begin{array}{l}\text { Management } \\
\text { commitment to } \\
\text { safety }\end{array}$ & $\begin{array}{l}\text { Leadership is } \\
\text { committed to } \\
\text { safety; safety is } \\
\text { a priority. }\end{array}$ & $\begin{array}{l}\text { Leadership } \\
\text { committed to the } \\
\text { safety }\end{array}$ & & \\
\hline $\begin{array}{l}\text { Work } \\
\text { Environment }\end{array}$ & $\begin{array}{l}\text { Respectful } \\
\text { work } \\
\text { environment. }\end{array}$ & $\begin{array}{l}\text { Trust permeates } \\
\text { the organization }\end{array}$ & Teamwork & $\begin{array}{l}\text { Frontline } \\
\text { involvement in } \\
\text { safety }\end{array}$ & Teamwork & Just culture & $\begin{array}{l}\text { Deference to the } \\
\text { expertise with } \\
\text { the specific } \\
\text { safety issues }\end{array}$ \\
\hline Raising Concern & $\begin{array}{l}\text { Environment } \\
\text { for raising } \\
\text { concerns. }\end{array}$ & & $\begin{array}{l}\text { Internal group } \\
\text { processes, such } \\
\text { as peer support } \\
\text { for safety }\end{array}$ & $\begin{array}{l}\text { A supportive } \\
\text { environment for } \\
\text { speaking up } \\
\text { about safety }\end{array}$ & Speaking up & $\begin{array}{l}\text { Reporting } \\
\text { culture }\end{array}$ & $\begin{array}{l}\text { Reluctance to } \\
\text { simplify } \\
\text { interpretations }\end{array}$ \\
\hline Communication & $\begin{array}{c}\text { Significant } \\
\text { environmental } \\
\text { and safety } \\
\text { communication }\end{array}$ & & & Communication & $\begin{array}{l}\text { Communication } \\
\text { and feedback }\end{array}$ & Informed culture & \\
\hline
\end{tabular}




\begin{tabular}{|c|c|c|c|c|c|c|c|}
\hline Accountability & $\begin{array}{c}\text { Personal } \\
\text { accountability. }\end{array}$ & $\begin{array}{l}\text { Everyone } \\
\text { personally } \\
\text { responsible for } \\
\text { the safety }\end{array}$ & & $\begin{array}{c}\text { Everyone } \\
\text { involved in } \\
\text { safety }\end{array}$ & & Flexible culture & $\begin{array}{l}\text { Deference to } \\
\text { expertise with } \\
\text { the specific } \\
\text { safety issues. }\end{array}$ \\
\hline Attitude & $\begin{array}{l}\text { Inquiring } \\
\text { attitude }\end{array}$ & $\begin{array}{l}\text { Questioning } \\
\text { attitude } \\
\text { cultivated }\end{array}$ & & & & $\begin{array}{l}\text { Reporting and } \\
\text { informed culture }\end{array}$ & $\begin{array}{l}\text { Preoccupation } \\
\text { with failure } \\
\text { (seeking } \\
\text { potential threats } \\
\text { to the system) }\end{array}$ \\
\hline Risk Management & $\begin{array}{c}\text { Hazard } \\
\text { identification } \\
\text { and risk } \\
\text { management }\end{array}$ & $\begin{array}{l}\text { Technology } \\
\text { recognized as } \\
\text { complex and } \\
\text { challenging to } \\
\text { manage }\end{array}$ & $\begin{array}{l}\text { Perceived job } \\
\text { risk; safety } \\
\text { attributes and } \\
\text { behaviors }\end{array}$ & $\begin{array}{c}\text { Risk } \\
\text { appreciation }\end{array}$ & Risk perception & Informed culture & $\begin{array}{l}\text { Preoccupation } \\
\text { with failure } \\
\text { (seeking } \\
\text { potential threats } \\
\text { to safety } \\
\text { system) }\end{array}$ \\
\hline Operations & Work processes & $\begin{array}{c}\text { Safety } \\
\text { undergoes } \\
\text { constant } \\
\text { examination }\end{array}$ & $\begin{array}{l}\text { Safety systems } \\
\text { limit work } \\
\text { pressures to } \\
\text { trade off safety } \\
\text { for productivity }\end{array}$ & $\begin{array}{l}\text { Safety rules are } \\
\text { followed and not } \\
\text { sacrificed for } \\
\text { productivity }\end{array}$ & $\begin{array}{l}\text { Safety systems } \\
\text { limit job } \\
\text { demands to } \\
\text { trade off safety } \\
\text { for productivity }\end{array}$ & & $\begin{array}{l}\text { Sensitivity to } \\
\text { operations }\end{array}$ \\
\hline $\begin{array}{l}\text { Continuous } \\
\text { Improvement }\end{array}$ & $\begin{array}{l}\text { Continuous } \\
\text { improvement }\end{array}$ & $\begin{array}{c}\text { Organizational } \\
\text { learning is } \\
\text { embraced }\end{array}$ & & & & Learning culture & $\begin{array}{l}\text { Commitment to } \\
\text { resilience }\end{array}$ \\
\hline
\end{tabular}




\subsection{Research Framework: A System Approach to Achieving HROs}

This research aims to explain and achieve safety by improving Organizational Reliability (OR) or, as some authors characterize it, "Collective Mindfulness.” To achieve this goal, it focuses on the practices and principles identified by HROT, which builds on Weick et al. (1999), Weick \& Sutcliffe (2001; 2015), and Sutcliffe (2011) and others. I first summarize the cultural and organizational characteristics of HROs (Sections 2.5.1). Then I discuss the earlier and the present interpretations and organize them in the theoretical framework for this dissertation, which is graphically illustrated in Figure 1 and described in Section 2.5.2. I subsequently present and critically discuss the elements of the framework in greater detail.

\subsubsection{Earlier Interpretations of HROs' Characteristics}

Since its beginning, research on HROs, such as Rochlin et al. (1987), has been concerned with organizations' cultural characteristics that operate with high levels of safety and reliability (Sutcliffe, 2011). Several broad themes emerge.

Approaches to Knowledge and Learning: According to Weick \& Sutcliffe (2001; 2015), HROs' culture is characterized as "generative" concerning the other types of organizational culture based on Westrum's typology (Westrum, 1993; 2004). This typology is developed according to how each cultural type treats the stream of safety-related information (see Table 3 below). As part of their culture, HROs actively seek information and encourage people to think, say, urge, negotiate, report, etc. While responsibilities are shared, failures are inquired about, and new ideas are welcomed and rewarded (Westrum, 1993; 2004). 
Table 3: Typology of Organizational Culture That Deals with Safety-Related Information

\begin{tabular}{|c|c|c|c|}
\hline Attributes & Pathological & Bureaucratic & $\begin{array}{c}\text { Generative } \\
\text { HROs Cultural Attributes }\end{array}$ \\
\hline Information Flow & Do not want to know & $\begin{array}{c}\text { May not find out, } \\
\text { "ignored." }\end{array}$ & Actively seek information \\
\hline Messengers & $\begin{array}{c}\text { Whistle-blowers are } \\
\text { "shot." }\end{array}$ & $\begin{array}{c}\text { Listened to if they } \\
\text { arrive }\end{array}$ & Trained and rewarded \\
\hline Responsibility & Evaded & Compartmentalized & Shared \\
\hline Bridging & Discouraged & Allowed but neglected & Rewarded \\
\hline Failure & Punished or concealed & Leads to local repairs & Leads to far-reaching reforms \\
\hline New Ideas & Actively crushed & Present problems & Welcomed \\
\hline
\end{tabular}

The culture of HROs provides the cognitive infrastructure for concurrent adaptive learning (Weick et al., 1999), resulting in continuously building and updating knowledge repertoires. This occurs through (1) particular types of training such as face to face, simulation, and video training (Roberts, 1990b), (2) reporting, investigating, and analyzing all types of failures (LaPorte et al., 1988), and (3) sharing information with other organizations (Turner, 1995; Reason, 1998). ${ }^{8}$

Being Proactive: The theorists of HROT further argue that accidents in complex systems are preventable through proactive processes that enable significant anticipation and containment of catastrophic errors (Rochlin, 1996; La Porte, 1996). HROT, therefore, takes the optimistic view that accidents do not only happen but occur through the accumulations

\footnotetext{
${ }^{8}$ Notably, perspectives on learning, namely learning by trial-and-error, have changed over time. Initially, HRO research emphasized a total elimination of failures and the absence of learning by trial-and-error (Weick, 1987), while later characterizations tolerate the inevitability of errors and acknowledge the need for limited trial-and-error learning (LaPorte \& Consolini, 1991).
} 
of latent errors and early signals (Weick et al., 1999) that are fundamentally discoverable. HROs implement this approach by integrating doubt as a mindset so that people stay preoccupied with anomalies and latent errors and are reluctant to simplify interpretations (Weick et al., 1999). This mindset mitigates the tendency to become complacent and, instead, causes people to remain alert and sensitive to operations. Because errors can occur and evolve into something larger, they require continuous detection and immediate correction. Consequently, HROs encourage all people to raise their voices and concerns without fear of blame and accusations (Rochlin, 1993). In fact, HROs have reward systems for people who participate in reporting any issues or concerns (Roberts, 2003; Weick \& Sutcliffe, 2015).

Structural Flexibility: Structural flexibility allows the response to unexpected events by migrating the decision-making process to those with unique expertise regardless of their rank and authorization. This characteristic is also known as safety on the front lines (Vogus et al., 2010). It is enabled by workers who understand the technology and procedures and develop specific actions and practices to cope with emergencies (Leveson et al., 2009).

Redundancy: In contrast to NAT, which considers redundancy in complex systems ineffective or even risky (Perrow, 1984), HROT promotes redundancy as an integral approach to achieving resiliency, safety, and performance (La Porte, 1996; Roberts, 1990; Weick et al., 1999; Weick \& Sutcliffe, 2015). Redundancy is defined as: "the ability to provide for the execution of a task if the primary unit fails or falters-is a necessity for highreliability organizations managing activities sufficiently dangerous to cause serious consequences in the case of operational failures" (Rochlin et al., 1987, p. 84). HROs use 
technical redundancies such as backup computers when those that are in use fail, human redundancies such as backup personnel to perform a given safety task when those in front lines lose control (Roberts, 1990b), as well as organizational redundancies to support the decision-making process in particularly trying conditions (Weick et al., 1999). These mindsets, learning strategies, and organizational design choices result in a unique safety culture that reduces the rate of unexpected events, such as accidents, increases the organization's ability to bounce back from disturbances, and increases productivity (Roberts, 1990).

In HROs, these characteristics (organizational learning, flexible structures, a proactive mindset, and redundancies) are implemented through unique principles, which sometimes also labeled as pillars, elements, strategies, practices, or cognitive processes (Weick et al., 1999; Rochlin, 1996; Weick \& Sutcliffe, 2015). Many of these characteristics were already highlighted in the concluding remarks of the first project on HROs, which reflected on the observed organizational patterns of the three studied organizations (LaPorte et al., 1988). Later, additional studies by the original theorists and others (Roberts \& Rousseau, 1989; Roberts, 1990; Roberts \& Libuser, 1993; LaPorte \& Consolini, 1991; La Porte \& Consolini, 1998) described similar (though not identical) principles. These early principles of HROT included the following:

Management by Exception: Managers are focused on long-term strategic decisions. While they monitor operations, they only get involved in the operational decisions as needed. Deference to Expertise During Emergencies: During emergencies, the decision-making 
process migrates to those with a high level of knowledge, regardless of their rank or authority, to ensure quick action taking without the need to seek higher rank approvals.

Continuous Training: Training provides knowledge and personal skills to the operators and managers to enable them to deal with the system's complexities and build trust and credibility among the workforces. As a result, people share the senses that everyone involved is competent enough to take responsibility and do their job correctly.

Communication of Safety-Critical Information: Operators are fully and promptly informed, especially in times of emergencies. To achieve this, HROs design multiple channels for sharing safety-critical information.

Redundancies: As described before in the general characteristics section, redundancy is a backup that provides a system of checks-and-balances for identifying risks before they occur or before they become out of control.

\subsubsection{The Specific Characteristics of HROs: A Present-Day Framework}

Beginning in 1999, the early characterizations of HROs described above became less pertinent because Weick et al. (1999) and Weick \& Sutcliffe (2001) defined five new fundamental principles more descriptive of the essence of their finding and more generalizable across multiple studies. These new principles cover both earlier and later characterizations of the HROs and include preoccupation with failure (e.g., by being proactive), reluctant to simplify (e.g., by designing redundancies), sensitivity to operations (e.g., by communicating safety critical-information), commitment to resilience (e.g., by continuous training), and difference to expertise (e.g., by designing flexible structures). 
Each of these principles will be defined and discussed in more significant detail in the next Section 2.5.3.

These five principles form two reciprocally interacting groups based on the nature and the objectives of practicing each principle. The first group consists of preoccupation with failure, reluctance to simplify, and sensitivity to operations, which are continuously interacting with each other to develop an organizational capability to discover unexpected events as a system of multiple barriers to defend the organization and its people from any potential threats. This group supports unique practices that include but are not limited to continuous searching for, discovering, controlling, and solving even small issues within time and before they accumulate and augment into broader issues. This developed capability is also known as mindful anticipation, which will also be defined and discussed in more detail in Section 2.5.4.

The second group comprises commitment to resilience and deference to expertise, which are also continuously interacting with each other to create an organizational capability to manage unexpected events. This capability enables people to cope with unforeseen threats, solve broader issues when they become out of control, minimize their effects if they lead to safety-critical events, and quickly return to operations' normal conditions. This organizational capability is also known as mindful containment and will be defined and discussed in Section 2.5.4.

The outcome of these two interrelated and interdependent groups of principles is organizational reliability or, according to some authors, collective mindfulness. It is an ultimate objective that results in a strong safety culture and a high operational performance 
level. Figure 1 is a graphical illustration of the research framework that consists of the briefly discussed above activities. The following sections define and discuss each element of the framework in greater detail.

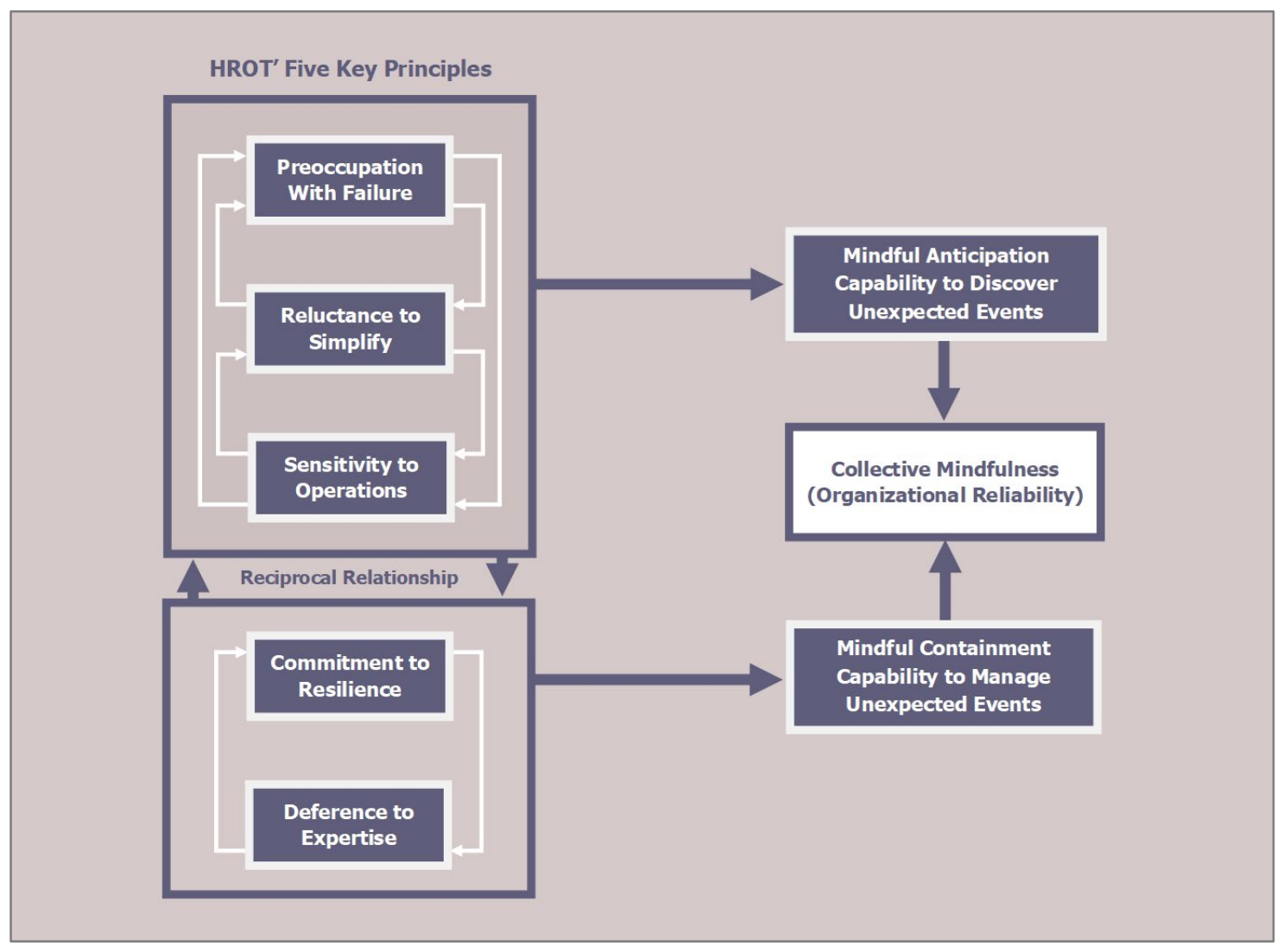

Figure 1: Research Framework

\subsubsection{The Five Principles of HROT}

The following subsections include definitions and extensive discussions around the five HROT principles, including how each principle is characterized in the earlier and later characterizations discussed in the previous sections. 


\subsubsection{Preoccupation with Failure (PWF)}

Preoccupation with failure is a chronic precaution that motivates a proactive and preventative inspection of potential weaknesses in the organization and treats any type of failure or near-miss as an indicator of potentially large-scale issues (Sutcliffe, 2011). This principle is central for the HROs: it leads to a precautionary approach to constantly maintain readiness on an operational level. It also results in a mindset that suspects anything may go wrong at any time, which mitigates the tendency of becoming complacent (Weick \& Sutcliffe, 2001; 2015). The focused attention on possible failure (Weick et al., 1999), even though failures in HROs are rare, is chosen because simple errors could be indicators that there is something wrong within the system that could lead to more severe events (Aven \& Krohn, 2014; Lekka, 2011). As a result, the members of HROs are concerned when things go smoothly because this may mean that they have not caught everything (Sutcliffe, 2011).

This mindset results in three actions, each of which requires rigorous attention: (1) Detecting small and emerging failures, (2) anticipating and specifying significant mistakes, and (3) acknowledging that people have incomplete knowledge of the situation, the environment, and their group (Weick \& Sutcliffe, 2015). It is also closely linked to organizational learning because it requires that the members of the organization must have the necessary knowledge of (1) the system details to construct logical thinking within the expectations domain of the processes they operate and (2) failure knowledge so that people understand how failure can evolve and can cause the system or subsystems to collapse. Therefore, HROs develop and sustain repositories of failure, such as experts and people 
with domain-specific knowledge and written failure, incidents, and accidents reports as sources for information inquiry.

However, preoccupation with failure can negatively affect the member's confidence in their competencies and knowledge (Lekka, 2011). It can exhaust the organization's financial resources on training and continuous learning (Leveson et al., 2009). Moreover, since this practice requires ongoing reporting of errors and safety-critical events, organizations that do not endorse a culture of reporting issues and raising concerns will result in members that fear such actions to avoid punishment and accusations (Weick \& Sutcliffe, 2001).

\subsubsection{Reluctance to Simplify (RTS)}

Reluctance to simplify is the tendency to question assumptions, think critically, and create a detailed picture of the operations (Sutcliffe, 2011). Simplification might be required for order and clarity (Weick \& Sutcliffe, 2015), but simplifying things too much or too quickly may ignore and obscure significant information needed for problem-solving (Weick et al., 1999). Simplification weakens people's defenses and the capacity of undesired consequences they predict (Sutcliffe, 2011) since it increases the likelihood of the unexpected (Weick \& Sutcliffe, 2015). HROs limit simplifications by widening the base of the precautions they implement.

HROs emphasize constant doubt regarding all aspects of the operations (Lekka, 2011) and systematically considers a unique context of the problem based on unique solutions by discouraging routine prevalent "best practice" solutions to problems (Hales \& Chakravorty, 2016). The necessary exercise to reduce simplification is to distinguish things 
based on the specific context that they are falling into and the details that they are providing.

To accumulate and process accurate information, HROs organize for action-based inquiry, which is a pattern for generating and gathering information from actions, particularly from people who have already been confronted with unexpected events (Roberts et al., 1994). The information generated through this mechanism requires recursive interactions between interpreting and the continued updating of the information (Weick et al., 1999). Reluctance to simplify is also embedded in the structural and organizational design. HROs organize for variety, wherein a complex system, complexity is the only way to cope with the complex environment and sustain healthy conditions.

It has been acknowledged that diverse groups have a more comprehensive range of information than those in homogeneous groups. Moreover, when organizational variety is coupled with redundancies (i.e., technical, human, and organizational duplications and backups in the system), a sense of discrediting is developed in the organization, causing significant information to be interpreted with fewer simplifications, generalizations, or normalizations (Weick \& Sutcliffe, 2001). However, designing a redundant system can breed complacency, which may reduce the principle of preoccupation with failure (PWF) (Weick et al., 1999). Complacency can also cause decision-makers to overestimate their systems' reliability (Pettersen \& Schulman, 2016; Schöbel, 2009), which may cause them to allocate insufficient financial resources for safety improvements (Weick et al., 1999). It can also affect the level of sensitivity to operation (STO) because it reduces the frontline's concentration on ongoing operations (Weick \& Sutcliffe, 2015). 
Moreover, redundancy in how information flows can confuse which source of information the organization members should use and cause them to disregard communication channels that were deliberately designed to ensure that information flows to where it is needed. This negative effect of redundancy is not commonly discussed in the literature, which worth investigating and future research.

\subsubsection{Sensitivity to Operations (STO)}

Sensitivity to operations refers to the collaboration and constant sharing of information that enables the organization members to develop a system view of the operations. This permits them to interrupt and adjust operations to prevent errors from accumulating and evolving into unmanageable issues (Sutcliffe, 2011). STO emphasizes situation awareness or what it is called in the navy as "having the bubble" (Weick et al., 1999), which refers to the attention of the ship's overall conditions at the moment (Hales \& Chakravorty, 2016). While unfolding, vigilance and focus on the situation are crucial; even small gaps in the operations require individual attention (Weick \& Sutcliffe, 2015). STO furthermore prevent anomalies from becoming the norm over time (Sutcliffe, 2011). The accumulation of unmanageable anomalies, notably when they do not cause critical issues in the short run, can become the standard over time following what is known as normalizing deviance (Goff et al., 2015). Therefore, anomalies must be caught and treated while still manageable before they evolve into a catastrophic accident (B\&W Pantex, 2008).

Operations can fail when operators' attention and mental concentration on operations deviated due to various factors such as ignorance, casualness, and general distractions (Weick \& Sutcliffe, 2001). To remain sensitive to the operations, operators must sustain 
dynamic situational awareness about their surroundings by picturing the operations as an integrated map. This requires that they construct and maintain a mental representation of operations that integrates diverse inputs, information sensors, and remote observation with the system's real-time status and performance (LaPorte \& Consolini, 1991; 1998). However, this can exhaust operators when they continue to concentrate on the same task for a long time because human cognition is limited.

One of the most adverse factors to sensitivity to operations is when organizations pay more attention to efficiency than performance. Focusing on efficiency often leads to increases in time and production pressure, which negatively affects people's cognition to stay focused; thus, they lose attention since they are busy accomplishing top management strategies and production plans. Pressured operators tend to ignore (Rerup, 2009), generalize, and simplify important and complex signals (Weick et al., 1999). Working in constantly pressured operations and continuous action without reassessing the overall situation causes people not to notice cues for abnormalities. It leads to so-called dysfunctional momentum, which eventually leads to a drift of performance from reliability to flawed norms. This phenomenon is known as the normalization of deviance (Vaughan, 1996).

To maintain STO, HROs intentionally plan interruptions and force adjustments to operations so that operators are returned to a sensitive mode in which they are aware of the operations (Sutcliffe, 2011). Another critical element of this principle is the development of heedful interrelationships among the organization's members. Such relationships are characterized by a collective mindset that thinks of individual actions as part of a larger 
pattern of shared activity and allows the organization members to understand how it fits into that larger pattern.

\subsubsection{Commitment to Resilience (CTR)}

Commitment to resilience refers to developing and fostering competencies that enable the organization to vigilantly notice, contain, and learn from errors that have already occurred and swiftly resume normal operations (Sutcliffe, 2011). It is also known as the organizational capability to recover a dynamically stable state for ongoing operations after a significant shock or stress (Weick et al., 1999; Pettersen \& Schulman, 2016). This means that HROs can be stretched without breaking as its design is elastic, and the organization can recover its shape quickly (Weick \& Sutcliffe, 2015).

Resilience is a result of organizational learning, as well as organizational design. According to Pettersen \& Schulman (2016), there are three types of resilience: (1) precursor (i.e., monitoring the operations), (2) restoration (i.e., rapid actions to resume operations), (3) and recovery (i.e., putting damaged systems back together). Performing these resilience types requires a very well trained, uniquely skilled, confident, and persistent workforce that can actively manage events (Sutcliffe, 2011), learn thoroughly from their actions (Vogus \& Hilligoss, 2016), negative feedback, and others through ad hoc networks (Weick et al., 1999). Accordingly, "sensemaking" is central. It describes the process that enables people to interpret a situation and come up with explanations and expectations that can also be communicated to other organization members. It thus is a social construction that creates enough certainty and order in a trying situation that decisions can be made. In dynamic conditions, sensemaking is an ongoing process: people 
need to continually assess and understand what is happening to prepare themselves for what will happen next. This process of continuous assessments and calibrations is characterized as "rebuilding the evolving present" (Weick, 1987).

CTR uses and produces knowledge: it provides the foundational knowledge that enables people to improvise and cope with emergencies. It creates a new experience as people witness sensitive and trying situations and come to a closure. As organizations authorize skilled people to improvise when needed, CTR provides them with the means to deal with and learn from failure, which builds coherent responses and action repertoires that consists of different levels of information richness, collaborative practice, and pro-active focuses.

\subsubsection{Deference to Expertise (DTE)}

Deference to expertise (DTE) refers to the organizational strategy of shifting the decisionmaking process to people with unique and specialized knowledge. Such as people on the frontline of an emergency whenever the situation requires (Weick \& Sutcliffe, 2001; 2015). This means that frontline people can take action without waiting for the hierarchy's approval (Lekka, 2011; Sutcliffe et al., 2016). This means that the structure of an HRO change during emergencies and is configured according to the problem at hand, which often enables HROs to develop unanticipated solutions (Roberts et al., 1994). However, deferring to expertise does not always lead to decision making, but sometimes it generates sensemaking, advice, and interpretations only.

In practice, DTE is an organizational principle that demands of managers not to answer questions that are outside of the scope of their expertise but to rely on experts who have the necessary knowledge in providing the proper solution (Aven \& Krohn, 2014), 
sometimes characterized as "respectful yielding." These experts are typically individuals who have earned expertise and their peers' respect (Weick et al., 1999; Weick \& Sutcliffe, 2015). HROs trust and highly value their true expertise, which depends on how many years they spend in a specific field, but on how many times they have been involved in and the degree of their involvement in emergencies and trying conditions (i.e., their "compressed experience") (Weick \& Sutcliffe, 2015).

To implement the principles of DTE, organizations need to have a culture of trusting in, having respect for, and yielding to expertise. Moreover, members of the organization need to be mindful of their knowledge and limitations and aware of other people's competence (Schöbel, 2009). However, the extent to which DTE is (and should be) practiced is contextdependent. For example, in some industries, such as health care, the decision-making process is based on education level, not just based on field experience.

\subsubsection{Organizational Reliability (Collective Mindfulness)}

According to Weick et al. (1999) and others such as Vogus \& Sutcliffe (2012), the three studied HROs have successfully achieved and sustained a high level of organizational reliability (OR) by effective implementations and equal attention to the five interrelated HROT key principles that I defined and discussed above. They suggest that bringing the five HROT principles together would develop a joint capability of organizational reliability or "collective mindfulness," as a conceptual construct that combines expectations, sensemaking, organizing, and managing. Collective mindfulness is defined as: "a means to increase organizational reliability and mitigate the adverse potential of unexpected, so-called black swan events (Taleb 2007) and is characterized by five mindful 
behaviors, including continuous learning from failures and the willingness to consider alternative perspectives" (Carlo et al., 2012, pp. 1081-1082).

Collective mindfulness points to the many ways that the five cognitive processes (principles) interact to create and sustain organizational knowledge and learning. It reflects an organization's capabilities to manage its recourses and detect, identify, and quickly respond to any potential threats (Weick et al., 1999). However, organizational reliability or collective mindfulness doesn't lead to the desired level of reliability by following checklists or adhering to controlling techniques to guarantee steadiness, but rather, when the only certainty is uncertainty, readiness and swift adaptability for emergencies are most essential fundamentals for reliable performances (Gebauer, 2013).

According to Sutcliffe (2011), two competing capabilities are reciprocal interrelationship to achieve organizational reliability: Prevention or mindful anticipation and resilience or mindful containment. On the one hand, anticipation refers to building a capacity to predict and prevent potential threats or unwanted events in their incubation period ${ }^{9}$ (Turner, 1978) before they evolve and become unmanageable or turn into a disaster (Roberts, 2003). Mindful anticipation is achieved when organizations equally practice each of PWF, RTS, and STO. These three principles are interrelated and interdependent through various shared cultural characteristics and practices (Weick et al., 1999; Weick \& Sutcliffe, 2015).

\footnotetext{
${ }^{9}$ Incubation period is defined as "a chain of concealed errors and other partially understood events buildsup in a way that is at odds with the existing beliefs and norms about hazards" (Pidgeon \& O'Leary, 2000, p. 16).
} 
On the other hand, containment refers to building a capability that copes with the effects of unexpected events after they occur and quickly return to normal operations (Sutcliffe, 2011). Mindful containment is attained when organizations equally practice each of CTR and DTE, which are also interrelated and interdependent, through which several characteristics are shared (Weick et al., 1999; Weick \& Sutcliffe, 2015). Furthermore, a system of collective mindfulness consists of components that are connected similarly to a neural network as Weick \& Roberts have urged in the following statement (Weick \& Roberts, 1993, p. 359):

"What connectionism contributes to organizational theory is the insight that complex patterns can be encoded by patterns of activation and inhibition among simple units, if those units are richly connected. This means that relatively simple actors may be able to apprehend complex inputs if they are organized in ways that resemble neural networks. Connectionists also raise the possibility that mind is "located" in connections and the weights put on them rather than in entities. Thus, to understand mind is to be attentive to process, relating, and method, as well as to structures and content".

Weick \& Roberts (1993) urges that the concept of collective mindfulness stems from the ideas of artificial intelligence to describe organizations as (1) Mental entities that are capable of thought, and (2) as distributed information processing systems and their association with the theories of connectionism" that are embodied in the so-called "neural networks," and their applications in organizational theory.

Inspired by the above descriptions, each principle consists of various numbers of causally related (connected) components that form its construct and represent its attributes. These 
components are also in causal relationships with the components of the other principles within the same group (i.e., the group of mindful anticipation or mindful containment) and the components of the other group's principles. In other words, HROT five principles form a network of causally related components that build the construct of organizational reliability or the collective mindfulness.

Collective mindfulness assesses the extent to which leadership endorses practices and rules that enable heedful ways of acting, thinking, and organizing (Ray et al., 2011). Therefore, it is a "top-down" process similar to any culture (Vogus \& Sutcliffe, 2012).

\subsubsection{Interim Conclusion}

The theory of High Reliability Organizations (HROT) provides five cognitive processes (i.e., the five fundamental principles) that allow organizations, particularly those with high hazard operations, to develop organizational capabilities to discover and manage unexpected events. If consistently implemented and maintained, these capabilities result in organizational reliability or - what a newly emerged research stream - labels collective or organizational mindfulness. In a dynamically changing world characterized by surprise events, this is an ultimate objective for many organizations' safety programs in different industrial fields.

Collective mindfulness is achieved by interplaying two groups of organizational principles (i.e., mindful anticipation and mindful containment). It empowers organizations to strengthen and sustain a safety culture while maintaining a high level of operational performance. The resulting high level of organizational reliability does not just result in low failures or accidents rate. It also creates an organizational competence and readiness 
to cope with unexpected events and mitigate their often damaging effects. Therefore, increased organizational reliability is understood to be a function of improved performance of the five HROT principles, which are dynamically interrelated.

While HROT principles and their systemic nature have been discussed in the literature for over thirty years, there is a lack of a nuanced discussion of what these principles comprise and how they are interrelated to form a network of system components. For the most part, the HROT principles have been discussed as processes that should be implemented concurrently and to the most significant degree possible. This will result in positive outcomes. However, given the current descriptions of these principles and based on attempts to implement them in different industrial fields, it appears that there may be tradeoffs and that implementing some principles may be counterproductive to achieving others. This means that the implementation process and the appropriate degree to which these principles are implemented are most likely dependent on multiple factors that occur internally (e.g., business strategies, policies, etc.) and externally (e.g., government regulations, production demand, etc.). However, there is no comprehensive system model to provide a holistic understanding of these dynamics to date.

Moreover, some of the contributing factors are explicitly discussed in the literature; other factors were merely mentioned or implicitly deliberated. Therefore, developing a system model based on these principles requires a thorough investigation to identify the factors that foster the implementation processes and those impeding them. 


\subsection{Applying HROs in Real-World Systems}

While HROT is a well-rounded and accepted theory, it nevertheless poses the question if the theory of five principles can help improve safety culture in practice. There are two broad categories of concern: (1) Are the five HROT broadly applicable in organizations with different characteristics than the organizations included in the original research? For example, initial research was done on nuclear power plants and aircraft carriers - do findings also apply to hospitals? And (2) To what extent do HROT principles improve safety culture and outcomes and how considerable are the improvements? The following sections review empirical research to answer these questions.

\subsubsection{Applicability of HROs in Different Systems and Industries}

Early HROT research was mainly focused on safety-critical organizations (Harvey et al., 2016 ) with unique characteristics, which were identified by Roberts \& Rousseau (1989) as Hypercomplexity, tight coupling, system designs that allow only one way to reach the goal, extreme hierarchical differentiation, large numbers of decisions, a remarkable degree of accountability, high frequency of immediate feedback regarding decisions, compressed time frame, and more than one simultaneous critical outcome. These characteristics shaped the practices that HROT describes and explains. Accordingly, it was initially unclear if the same practices can be implemented in other organizations and if implementing them is beneficial for safety in their specific context (Leveson et al., 2009; Hopkins, 2007). For example, petroleum refineries, railroads, and many other industries have continuous processing facilities and time flexibility (Perrow, 1984), technologies with a high degree of predictability (Roberts \& Rousseau, 1989), the interactions among system elements are 
comprehensible (Lekka, 2011), the technology is well known, straightforward, and stable (LaPorte \& Consolini, 1991; Pettersen \& Schulman, 2016), and the public considers its hazards to be self-limiting (Rochlin, 1993). This means that risk can easily be identified and managed proactively, making them quite different from HROs who need to manage unexpected events.

Moreover, HROs seek nearly full knowledge of their technical aspects and relatively stable technical processes to foster system comprehension. This may make it infeasible to implement HROT principles in profit-making organizations (Leveson et al., 2009) because such organizations are continuously seeking technological advancement to create and sustain competitive advantages that cope with market uncertainties and pressures and may never have full knowledge of technical details (Marais et al., 2004; Lekka, 2011). For economic and regulatory reasons, naval aircraft carriers, aviation systems, and nuclear power plants replace technology at a much slower pace. However, HROs are known for their endorsement of technological advancement and system updates (Roberts \& Rousseau, 1989). More recently, these questions about the applicability of HROT principles to different organizations have given way to a more inclusive view: HROT research witnessed a shift in focus, terminology, and adaptability (Hopkins, 2007) as Weick \& Sutcliffe (2015) acknowledged the similarities between HROs and other organizations "all organizations develop precautions against these hazards that are set out in norms, regulations, procedures, rules, guidelines, job descriptions, and training materials. And all organizations accumulate unnoticed events that are at odds with accepted beliefs about hazards" (Weick \& Sutcliffe, 2015, p. 19). They also view the commonalities among all 
types of organizations as an excellent opportunity to inspire the non-HROs to intensively learn from HROs regarding how to achieve a successful and desirable business.

Consequently, there have been many attempts since 2001 to adopt and practice HROT principles in various industries, but most notably in healthcare (Vogus et al., 2010; Vogus \& Sutcliffe, 2007a; Madsen et al., 2006; Frankel et al., 2006). Implementations in healthcare faced considerable difficulties due to this industry's nature (Roberts \& Martelli, 2016), particularly in the decision-making structure. For example, it became apparent that designing redundancies in healthcare had adverse safety outcomes (Madsen et al., 2006) because people relied too heavily on somebody else in the system to catch mistakes. This indicates that implementing the principles of HROT is a context-dependent process, or in other words, dependent on each industry's specific characteristics, circumstances, and particular ways for adapting to this approach.

In addition to healthcare and related fields, such as drug rehabilitation (Cooren, 2004), HROT principles were also investigated in firefighting teams and fire management (Berardi, 2010; Black \& McBride, 2013), oil refineries (Lekka \& Sugden, 2011) offshore oil and gas (Aven \& Krohn, 2014), onshore natural gas (Paranhos et al., 2017), and the construction industry (Harvey et al., 2016 ). With a focus on reliability, more so than safety, the applications of the HROT five principals were also attempted in industries that are not particularly hazardous such as architectural design (Carlo et al., 2012), reliability seeking virtual organizations (RSVOs) (Grabowski \& Roberts, 2016), software industry (Vogus \& Welbourne, 2003), and commercial banks (Weick \& Sutcliffe, 2015). A key challenge in many of the reported implementations is a lack of knowledge about how HROT principles 
were implemented in the field. Some industries claimed that they were successful in practicing HROs, such as the U. S. nuclear industry (B\&W Pantex, 2008), U.S. Navy submarine and carrier aviation services, commercial aviation worldwide (Roberts \& Martelli, 2016), and NASA (Boin \& Schulman, 2008). Yet, it is unclear how they interpreted and implemented the principles and how successful they were at shifting culture towards improved safety and resilience (Roberts \& Martelli, 2016). Recent events, such as accidents in the US Navy and several high-profile plane crashes, also shed doubt on HROT principles' ability to maintain a safety culture.

\subsubsection{Assessing HROs in Practice}

The literature on high reliability organizations provides a rich foundation for the theoretical background, fundamental processes (key principles), and various qualitative and quantitative empirical studies that have examined, measured, and attempted to validate the concept of organizational reliability. Table 22 in Appendix A: Empirical Studies on HROs includes multiple empirical studies and applications of HROT principles in different research contexts. As presented in the table, most studies are qualitative. They have used various research designs and methods such as action research, case studies, interview-based research, observational studies, and the use of government documents (Sutcliffe et al., 2016). Qualitative studies thus provide a rich foundation for quantitative techniques, notably survey measures, such as unidimensional or multi-factor tests (Vogus \& Sutcliffe, 2012). These studies' commonalities mostly rely on the conceptualization of high reliability as a "mindful infrastructure," which was developed initially by Weick et al. (1999). This means that researchers ground their work on HROT and model collective 
mindfulness (or organizational reliability (OR) as an outcome that can only occur if all five fundamental principles are achieved, prompting Weick \& Sutcliffe $(2001 ; 2015)$ to describe them as a "package."

However, most studies are less interested in assessing the level of "HRO-ness" or achieved organizational reliability but aim to assess specific factors to the context and understand how they impact the HRO principles and collective mindfulness. Studies, for example, investigate the role of managing and avoiding conflict in healthcare institutions (Valentine et al., 2010), the impact of mindfulness practices (Hales \& Chakravorty, 2016), and the effects of huddle meetings (Goldenhar et al., 2013). This means that previous studies did not result in robust methods or measures that would help organizations to holistically assess how ready they are to become an $\mathrm{HRO}$ or to what extent they are an HRO already, making it difficult to track progress towards the goals of HROs. Moreover, these and other studies provide evidence that, as a result of contextual factors, HROT principles need to be implemented differently in different organizations and industries and may vary in the degree to which they promote organizational reliability. As a result, organizations require contextualized approaches for tracking their progress towards organizational reliability.

An additional complication is that industry and organizational contexts are not static but change over time, changing how the HROT principles affect reliability and render formerly successful HRO interventions less effective. For example, in the study performed by Ray et al. (2011), the authors operationalize and measure mindfulness among different leadership levels of US business schools and discuss that this can be used to gain insights into their organization's ability to weather crises - a more mindful leadership should be 
better prepared to manage detrimental events, such as reduced enrollment and resources. However, in practice, it appears highly unlikely that leadership would still have the same level of mindfulness in an environment with strongly declining resources when people leave, leadership changes, work pressures go up, and practicing mindfulness might fall victim to cost-cutting. This, and other existing measures, cannot currently predict and analyze the system's future state in response to external changes (e.g., enrollment decline)

and internal action (e.g., program cancellation, cost-cutting). Accordingly, present measures do not provide clarity about what an organization must do to remain reliable.

\subsubsection{Interim Conclusion}

HROs is a viable approach in any organization that seeks to become mindful in managing unexpected events regardless of the type and level of the operational hazard, including nontechnical organizations. Organizational reliability is often referred to as organizational mindfulness. However, the procedures of practicing HROs and the difficulties and challenges associated with the resulting outcomes differ from one organization to another, even within the same industry, and depend on the organizational and operational settings. This means that following the same procedures that other organizations have followed in practicing HROs would not lead to or yield similar outcomes. Therefore, management actions to practice HROs by designing and implementing managerial interventions, safety policies, strategies, and programs must be contextualized to the specific organizational and operational settings of each organization or industry.

Furthermore, available measures have been designed to assess the static state of organizations that aspire to become HROs; however, these measures lack the capabilities 
to evaluate and understand how far along organizations are on their path to being HROs and what should be done to get there. Because the operational environment, especially in safety-critical organizations, is dynamic and highly influenced by internal and external changes, current measures cannot predict systems' future state or assess particular situations based on various scenarios. That is why a system model based on HROT principles is needed to strengthen and sustain a safety culture.

\subsection{Research Context: The Offshore Oil and Gas Industry}

This research uses the offshore oil and gas industry to test, verify, contextualize, and assess the would-be developed theory-driven model. I selected this industry as a research context due to the constant and high rate of accidents and incidents and their often-devastating consequences, making this industry a significant safety concern for many years. Considering the interdependent and complex challenges in this industry, this research joins the stage of academic research to develop a practical tool that can become a means of decision support for this industry to manage and promote a safer work environment.

\subsubsection{Industry Overview}

For decades, the Gulf of Mexico has been one of the most lively and appealing exploration frontiers worldwide (Priest, 2008), which made offshore oil and gas one of the most developed industries in the world (National Academies, 2016). The factors that positively position this industry in this vital place are the age of and the continuously growing scale of the explorations. According to Morton (2016), the first large platform was built offshore in 1938 by both Pure Oil (now Chevron) and its partner Superior Oil (now ExxonMobil). However, as of November 2015, there are (33) mobile offshore drilling units operating in 
deep water (up to 10,000 feet), and more than $(2,500)$ platforms operating in shallow water (National Academies, 2016). This continuously growing scale to meet the increasing demand for oil and gas (National Academies, 2016) has established a long history in employing new and innovative technologies for commercial opportunities (Max et al., 2009). Based on the annual summary of production for the Gulf of Mexico, oil and gas production has reached $634,497,418$ BBLS of oil and 983,313,149 MCF of gas in 2018 (BSEE, 2019). However, for economic reasons, this industry has a proven history of prioritizing production over safety (National Academies, 2016), which has been a critical factor behind many large-scale accidents and incidents that have caused severe consequences (Zimmerman, 2012). The following sections discuss safety performance and culture in this industry.

\subsubsection{Safety Performance and Safety Culture}

The literature on the offshore oil and gas industry, including government reports and official data, shapes the conclusion that this industry is relatively unsafe. This conclusion is based on the following two major factors:

First: The offshore explorations based on the number and severity of accidents that have occurred on the Outer Continental Shelf(OCS) demonstrate that this industry is inherently hazardous (National Energy Board, 2014; Priest, 2008). In deep water, the operations occur in distant and usually unfavorable marine circumstances (Beatrice, 2011), where the chances of organizational and human errors are high due to the increasingly sophisticated technologies (National Academies, 2016). As a result, the offshore industry is listed among the most hazardous occupations in the world. 
Second: Despite all the improvements that have been made since the late of 1960 s to ensure safer operations through improving work procedures and practices, technologies, and government regulations (National Academies, 2016), official reports show that the accident rate, while fluctuating year-by-year, is still stubbornly high (National Academies, 2016; BSEE, 2015; 2016; 2017). Figure 2 presents the offshore incidents statistics ${ }^{10}$ between 2007 and 2018, as provided by the Bureau of Safety and Environmental Enforcement (BSEE, 2018).

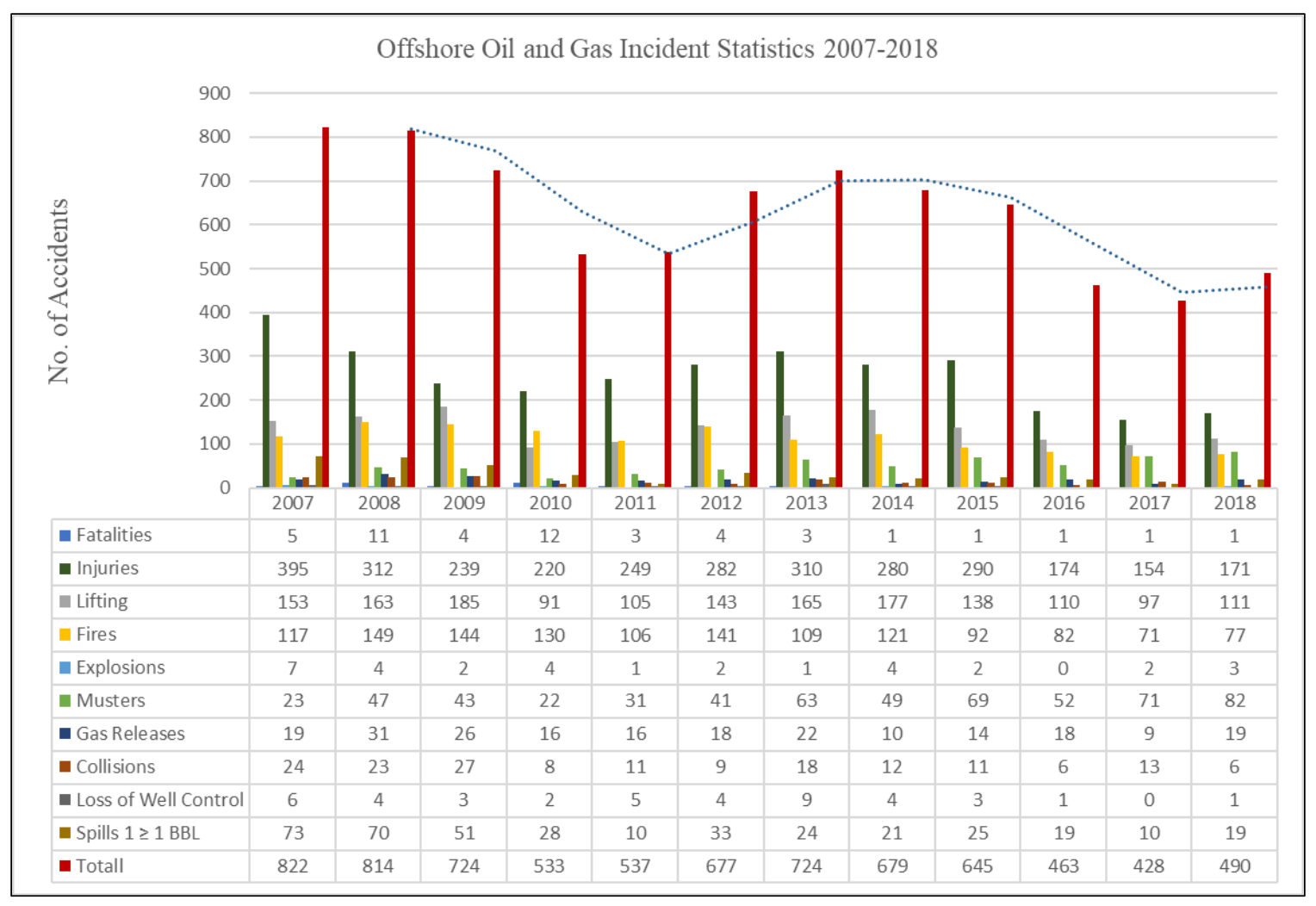

Figure 2: Offshore Incident Statistics 2007-2018

10 Information provided in Figure 2 above is taken from the BSEE's page on incident statistics (https://www.bsee.gov/stats-facts/offshore-incident-statistics). However, this data might be underreported since it does not match the data provided by the Center for Disease Control and Prevention (CDC). The latter shows some differences, particularly concerning the number of fatalities, as presented on this website: (https://www.cdc.gov/mmwr/preview/mmwrhtml/mm6420a4.htm). 
It is too early to tell if the reduction in the number of accidents in $2015,2016,2017$, and 2018 signals a persistent trend or simple fluctuation. Given the industry's nature, a single gap in the safety and risk management systems can lead to disastrous events that result in years with a high number of fatalities and catastrophic consequences such as the 2010 Deepwater Horizon.

There are various and interrelated factors that have led and continue to lead to accidents and disasters but, overall, the industry is recognized to have ineffective risk and safety management systems (National Energy Board, 2014; Hudson, 2007). Improving the situation requires a very careful examination of safety culture (National Academies, 2016), as several studies illustrate how it lacks in the industry. Fleming \& Scott (2012) studied the findings presented in the official investigation reports of fifteen major offshore accidents during 1980-2010. They found that a lack of safety culture was identified as the root cause that turned an accident into a disaster in twelve of these accidents. While five of these accidents were directly linked to safety culture: Piper Alpha (1988), Usumacinta (2007), Montara (2009), Sikorsky S-92A (2009), and Deepwater Horizon (2010), seven of them did not have direct cultural causes based on the study classification dimensions; however, poor safety culture was considered as a causal factor in these events as well (Fleming \& Scott, 2012).

The offshore industry's safety culture is derived from an organizational culture described as "male-oriented, macho, rough and tough, and can do" (National Academies, 2016, p. 20). There is a broad consensus among safety practitioners, scholars, and government that to foster and sustain a safe work environment, the offshore oil and gas industry requires 
compliance with the regulations and a fundamental transformation of safety culture, whereby organizations can move beyond compliance to operate safely.

\subsubsection{Challenges to a Strong Safety Culture}

Generally, any business or occupation can expect accidents and sometimes fatalities if it puts people to work in taxing and changing environments, operates sophisticated machinery, and increasingly relies on virtual work activities that often span across multiple companies and landscapes. However, Wright (1986) notes that accidents with fatalities are the norm of the routinely unsafe work practices in the offshore oil and gas industry. This is partly due to the constant time pressures on projects where the projects' profitability depends on quick and timely production (Priest, 2008). Furthermore, many companies rely on a temporary/contracted workforce for operations and disaster responses, which do not share the same training, experiences, and sometimes even language.

These challenges make ground observations even more difficult and create uncertainty about the state of the systems. According to Priest (2008), advancing such industry concerning safety is typically achieved by trial-and-error methods, which often leads to harmful consequences to the environment and can be fatal to workers. After interviewing former off-shore oil and gas employees, Priest (2008) came to the following conclusion (Priest, 2008, p. 144):

"Operators, and especially contractors, like the owners of mobile drilling vessels, did not overly concern themselves with safety. At times, they even cut corners. Safe processes and designs either did not exist or remained untested ideas in the minds of researchers. Facilities engineering on production platforms was a novel concept. Platforms were often 
stick-built with equipment squeezed or slapped together on the deck with little concern or foresight for worker safety."

The following are the significant challenges to fostering and sustaining safety culture in the offshore oil and gas (Priest, 2008; National Academies, 2016).

Work Environment and Shifts Schedule: Work environments in the oil and gas industry are stressful. The prevailing circumstances are unfavorable such as Darkness, excessive heat, cold, loud, and excessive noise. The shift schedule is long and stressful, with an average shift length of seven days on, seven days off. This leaves the workers tired and unfocused, which ultimately makes them susceptible to make errors that can lead to accidents and their potential consequences.

Labor Market: The turnover rate in the offshore labor market is increasing, and the use of contract employees is on the rise, which means that the industry frequently recruits inexperienced workers with a limited skillset who need more and adequate training. Unfortunately, even adequate training does not guarantee longevity in the workforce. Many industry segments have different and multicultural workforces that consist of employees with differing safety practices and varying educational backgrounds.

Operators, Contractors, and Subcontractors: During 1986 and 1994, the industry reported that $66 \%$ of the sector consisted of emerging smaller and independent operators. This took into consideration that those operators did not necessarily have an organizational structure for managing safety, or they may not have equally treated safety on the same level of importance. The relationships among operators, contractors, and subcontractors can confuse safety responsibilities, so deploying consistent practices becomes difficult. The 
varied perceptions towards each of those different parties' safety and economic interests create an operation that speaks in other voices regarding safety.

Leadership Commitment to Safety: Senior leaders and owners of organizations differ in their knowledge of, the obligation to, and engagement with the need to improve and maintain a strong safety culture. Leaders sometimes send wrong messages explicitly or implicitly, giving priority to production over safety, which makes it evident that their beliefs are oriented towards how the organization makes money whereby efficiency comes before performance.

Industry Nature: The nature of an industry is described as fragmented and diverse among companies and employees, making it challenging to design consistent and universal goals and implement them through industry-wide agreements. The operations occur in many different organizations (a mix of small and large companies) that vary as to their internal core values and safety assumptions.

Level of Safety Culture: Safety culture in this industry is still developing; however, a blaming culture and the lack of systems thinking even exists., This focuses on instant causes of failure (e.g., human errors) instead of the system causes, including the organizational culture.

Safety Regulations: Inspections for compliance will not guarantee safe offshore operations. Accountable organizations and progressive officials recognize the need to go beyond regulatory compliance by holistically supporting safety. One challenge for all regulators is to change inspectors' mindset from inspecting for compliance to advocating safety culture. 


\subsubsection{Interim Conclusion}

Various challenges impede the offshore oil and gas industry from developing and sustaining a strong safety culture. While some of these challenges are intrinsic in the technologies and environmental conditions surrounding the operations, others have been identified as cultural and organizational. To strengthen and sustain the safety culture in this industry, safety practices must be adapted to the context and go beyond compliance with rules and regulations. There is a need to implement and contextualize a comprehensive system approach for safety. This dissertation develops based on HROT five principles, especially since there is no specifically contextualized HROT principles model for this industry.

The contextualization procedure is essential in this work since practicing the five HROT principles is a context-dependent approach. Specific organizational, operational, and even environmental aspects of the operations are considered so that the expected outcomes from this entire process can reveal the industry's real-world capabilities and drawbacks. This helps industry decision-makers identify and understand the specific shortcomings that inhibit a high level of reliability.

Furthermore, since comprehensive HROT practical planning approaches for this industry are not available, the decision-makers continue to face difficulties in selecting adequate managerial interventions. When appropriately implemented, organizational reliability can be achieved, and confrontation with other interventions, initiatives, or safety programs are avoided. 


\subsection{Conclusion, Research Gaps, Objectives, and Questions}

High reliability organizations theory HROT provides a promising approach for developing and sustaining a positive safety culture in reliability-seeking organizations with the goal to reduce errors and avoid catastrophic events. Despite decades of research and extensive descriptions of HROT and its five fundamental principles, this approach remains elusive and difficult to achieve in many industries, particularly in the offshore oil and gas industry. Reliability is often misunderstood as an endpoint that reliability-seeking organizations can fully accomplish. Yet, reliability is not a state, but rather, a moving goal that organizations continuously aspire to reach. It is simple to lose sight of this goal because reliability is recognized by the absence of errors, and extended periods of achieving safe operations can lead organizations to overestimate their systems' reliability. As a result, they can reverse the focus from safety performance to efficiency and fail to pay attention to HROT principles as a dynamic set of interrelated and interdependent characteristics, activities, and responses that form a dynamic system.

To a large extent, the literature on HROT falls short in addressing the dynamic nature of HROT principles as systems. It insufficiently explains how these principles and their components are interrelated. Moreover, there are insufficient ways for measuring progress towards the goal of becoming an HRO. Instead, organizations use a checklist that assesses their static state rather than their dynamic readiness to cope with a wide range of unexpected events. The discussion above has furthermore shown that context matters and that the implementation of HROT principle in an industry such as the offshore oil and gas industry, which suffers from a deficient safety culture and poor safety performance 
measured by the accident rates, likely has to differ from the implementation in other industrial settings. Given the-state-of-the-art of high reliability organizations theory and challenges in the offshore oil and gas industry as a research context, the following gaps were found in the literature. This dissertation is designed to close.

\subsubsection{Lack of System Perspectives}

Despite the rich literature on how HROT theory is developed, progressed, and practiced in various industries, the literature falls short in providing methods or techniques for developing system models based on its five principles to assess the outcomes of the practitioners' decision-making process on safety-related practices. On the one hand, neither the contributing factors to the five principles are well understood. Their interdependencies are sufficiently investigated and weighted, nor is it clear how these principles are correlated to influence organizational reliability in real-world practice positively. On the other hand, there is limited knowledge regarding how the relationship between management actions and the five principles is established to guide the efforts towards a high organizational reliability level. Moreover, such relationships often encounter uncertainties regarding the appropriate model structure due to the variations in practitioners' opinions, which need to be managed during the modeling processes.

\subsubsection{Lacking Contextualization for Offshore Oil and Gas}

Previous studies on practicing the HROT principles conclude that plans and actual procedures, including requirements, challenges, and obstacles, and the anticipated outcomes differ from a context to another, depending on various organizational and operational factors. These factors include decision-making, communication patterns, 
failure and error reporting, training, operating shifts, work schedules, and many others. As a result, implementing HROT principles requires contextualizing the processes based on the context's real-world conditions under study. To date, there are no contextualized system models of HROT based on the offshore oil and gas organizational and operational settings. The lack of contextualization could result in wasted efforts to practice HROs, which may lead to a misunderstanding that the five HROT principles cannot be practiced in the offshore oil and gas industry.

\subsubsection{Lack of Practical Planning Approaches}

Implementing and contextualizing the principles of HROT require rigorous management actions and practical planning. Such planning includes the selection and implementation of proper managerial interventions that fit the general circumstances of the industry (i.e., how the operations are managed such as the number of work shifts) on the one hand and HROs practicing requirements (i.e., HRO-related practices such the continuous reporting of nearmisses and incidents) on the other hand. However, practical planning approaches to implement and contextualize HROT principles in the offshore oil and gas currently are not available. This limits the decision-makers' ability to select suitable interventions that work as a bridge to successfully achieve organizational reliability and reduce counterproductive effects that can results when confronted with other interventions and continue to achieve positive outcomes even when internal and external changes occur.

\subsubsection{Summary of Research Gaps, Objectives, and Questions}

Table 4 summarizes the research gaps, objectives, and research questions. 
Table 4: Research Gaps, Research Objectives, and Research Questions

\begin{tabular}{|c|c|c|}
\hline Research Gaps & Research Objectives & Research Questions \\
\hline $\begin{array}{l}\text { Lack of System Perspectives } \\
\text { There is no comprehensive system model that represents } \\
\text { the relationships between management actions, } \\
\text { "interventions," the five HROT principles, and } \\
\text { organizational reliability. This limits our understanding of } \\
\text { how organizational reliability can be achieved. }\end{array}$ & $\begin{array}{l}\text { - To develop a novel, mixed- } \\
\text { methods approach to modeling } \\
\text { the principles of HROT as a } \\
\text { Fuzzy Cognitive Map (FCM) } \\
\text { based on the published, peer- } \\
\text { reviewed research. } \\
\text { - To develop a comprehensive } \\
\text { FCM system model of HROT, } \\
\text { using the novel approach. }\end{array}$ & $\begin{array}{l}\text { 1. How can research publications on HROT be analyzed } \\
\text { to identify and represent relevant concepts and causal } \\
\text { relationships? } \\
\text { 2. How can the weight of causal relationships be } \\
\text { inferred from qualitative data in research } \\
\text { publications? } \\
\text { 3. How can information about concepts, causal } \\
\text { relationships, and relationship weights be used to } \\
\text { develop a general FCM model of HROT? } \\
\text { 4. How can uncertainty about the appropriate model } \\
\text { structure be managed in the modeling process? }\end{array}$ \\
\hline $\begin{array}{l}\text { Lacking Contextualization for Offshore Oil and Gas } \\
\text { Industry and organizational contexts influence how HROT } \\
\text { principles are implemented and how they impact OR, yet, } \\
\text { to date, no contextualization of HROT for offshore oil and } \\
\text { gas has occurred. This limits the ability to improve industry } \\
\text { safety performance through HROs practices. }\end{array}$ & $\begin{array}{l}\text { - To contextualize the FCM } \\
\text { model with the help of industry } \\
\text { experts so that it reflects the } \\
\text { unique context of the offshore } \\
\text { oil and gas industry. }\end{array}$ & $\begin{array}{l}\text { 5. How can the general FCM model of HROT practices } \\
\text { be contextualized to the specific settings of offshore } \\
\text { oil and gas operations? }\end{array}$ \\
\hline $\begin{array}{l}\text { Lack of Practical Planning Approaches } \\
\text { There is no comprehensive approach to supporting HROs } \\
\text { planning. Decision-makers lack support in selecting } \\
\text { interventions that (1) can be expected to improve } \\
\text { organizational reliability, (2) are synergistic with other } \\
\text { planned interventions to improve reliability, and (3) are } \\
\text { robust under changing conditions. }\end{array}$ & $\begin{array}{l}\text { - To evaluate the FCM model as a } \\
\text { tool for planning HROT } \\
\text { practices. }\end{array}$ & $\begin{array}{l}\text { 6. How can the contextualized FCM model of HROT } \\
\text { practices be used to support safety decision-makers in } \\
\text { the offshore oil and gas industry? }\end{array}$ \\
\hline
\end{tabular}




\section{Methodological Foundation}

This work uses various methods over multiple phases to close the identified research gaps and answer the research questions. In this chapter, I first provide an overview of the methodological selection, then I review the research methods, including Fuzzy cognitive maps (FCMs), Thematic Analysis (TA), Thematic Networking (TN), Thematic Proximity (TP), and Exploratory Modeling and Analysis (EMA). The chapter also presents techniques for model testing for verification and strategies for model simulation and scenario planning.

Figure 3 is a graphical illustration of how these methods will be presented and integrated to create, test, and simulate the research model.

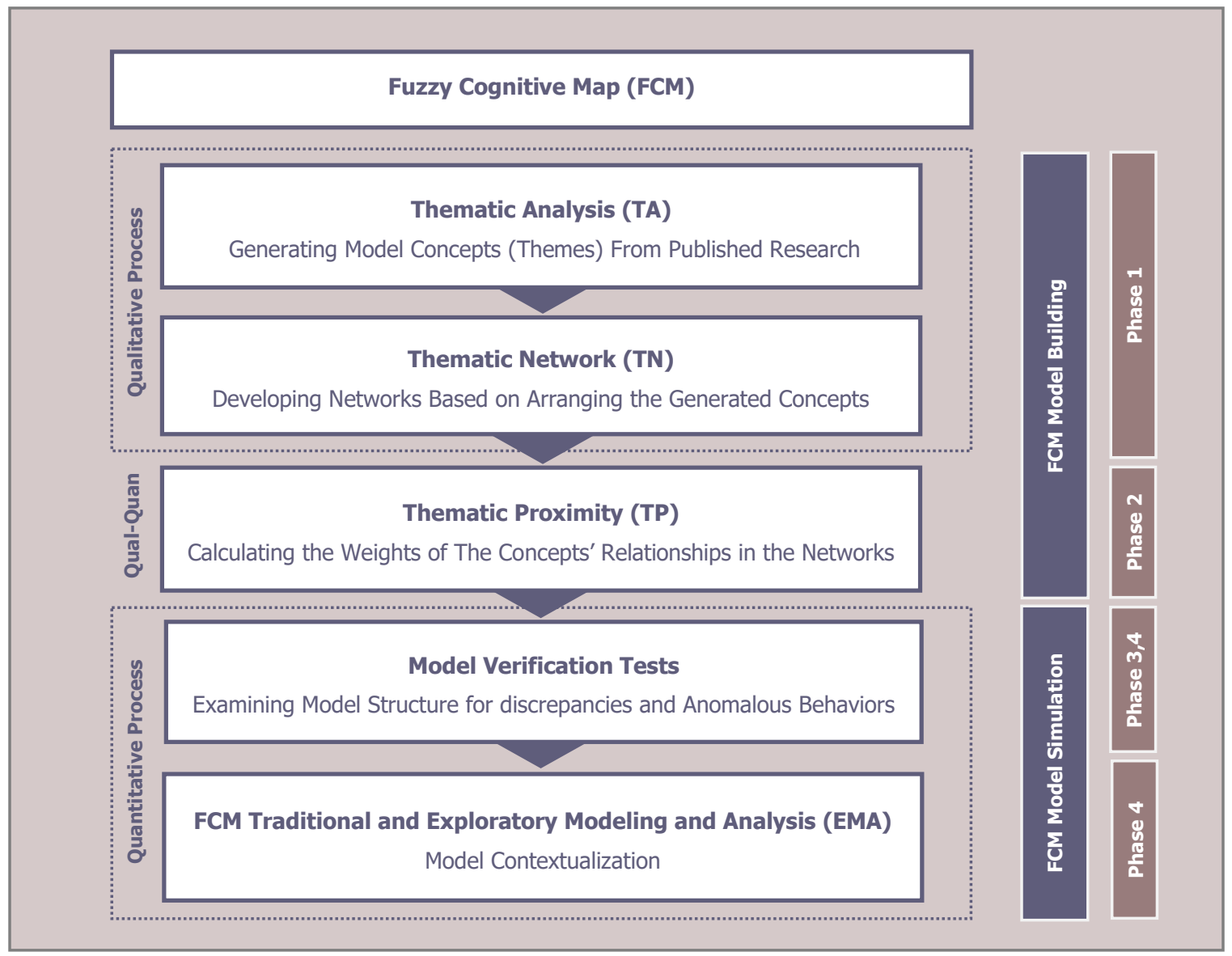

Figure 3: Methodological Foundation 


\subsection{Methodological Selection}

The overall objective of this research is to build a practicable theory-driven system model of organizational reliability based on FCM that can be used to simulate the effects of safetyrelevant activities on organizational reliability. The model will support organizations in the offshore oil and gas industry in their decision-making processes regarding safety-related practices, policies, strategies, and interventions. Creating the model requires extracting insights from published research, which is mostly qualitative, converting these insights into cognitive maps that show concepts and causal connections, and translating the cognitive maps into quantitative FCM models.

A variety of content-based methodologies exist to support the first step, namely the translation of insights from text documents (e.g., interviews or published research) into cognitive map structures in ways that capture concepts, meaning, and causal relationships between them. For example, Carley (1997) proposed an automated approach of a two-steps process to build a cognitive map using survey instruments, observation, and archival means to extract and represent mental models. The first step is to filter the text based on a predefined list of concepts, while the second step is to set a text size window to automatically generate maps from the filtered text. Although this is a fast approach to extract and represent mental models, it can create relationships among contiguous concepts that are not necessarily related. Moreover, this approach does not provide a means to measure the strength of the causal links in the developed maps.

Nadkarni \& Shenoy (2004) proposed a systematic procedure for creating structures for Bayesian Belief Networks, which share some similarities with FCM. This procedure 
consists of a four-step process: (1) data elicitation by conducting open-ended interviews, (2) derivation of a causal map ${ }^{11}$, (3) modifying the original map, and (4) assessing the parameters. This approach is quite different from the one proposed by Carley because it was conducted manually using both exploratory and confirmatory methods. It is entirely dependent on subject matter experts, who are charged with determining the causal map and assessing its parameters, based on their knowledge. Thus, the expert provides a (weak) link between published literature and the resulting map structures.

Alizadeh \& Jetter (2017) proposed a content-based approach to extract the FCM model from published research instead of conducting interviews. The process consists of five steps: (1) drawing the raw FCM based on the original text, (2) consolidating identical concepts, (3) adopting consistent terminologies for conceptually similar concepts, (4) tuning the granularity for concepts and sub-concepts, and (5) identifying and closing the gaps in the map, where the modeler hypothesizes connections to the concepts in the model that are left isolated to complete the collective FCM. However, this process does not provide a technique that resolves the issue of assigning weights to the causal relationships in the developed FCMs. In fact, in a follow-up study, Alizadeh (2018) experimented with the difficulties with an online survey that assigns weights to the FCM edges generated by the discussed process. Although participants were researchers in the field, the study became problematic for the recipients because they found the questions difficult, out of their area of expertise, or too time-consuming to answer.

\footnotetext{
11 This step also consists of a four steps process: (a) Identifying causal statements, (b) developing causal phrases, causal connectors, and causal effects, (c) designing a coding scheme, and (d) creating the final coded causal maps.
} 
In conclusion, several approaches have been used to extract knowledge and build causal model structures. The dependence on subject experts in assigning weights for the causal relationships in such models is highly problematic. Accordingly, this research develops and uses a different methodological approach, through which it (1) extracts knowledge from published research texts, using a semi-manual procedure to ensure that knowledge is rigorously and thoroughly captured, the concepts and their meanings are uniquely defined, and the causal relationships are established, and (2) facilitates a quantitative estimation of the causal relationships weights between concepts based on the same analyzed texts. It thus provides an alternative method to survey subject-matter experts to assign the weights. This approach consists of thematic analysis, thematic networks, and a newly developed approach that uses thematic proximity for assigning causal weights. The outcome is the FCM model based on published research on HROT five principles. The following sections discuss each method and the rationale behind selecting each method to conduct this research.

\subsection{Fuzzy Cognitive Maps (FCMs)}

Fuzzy Cognitive Map (FCM) is a system thinking technique to model complex matters with cause and effect relationships and dynamic behaviors (Jetter, 2006; Jetter \& Kok, 2014). FCM was invented in the artificial intelligence field by Kosko $(1986 ; 1988)$ to employ cognitive maps with dynamic behavior through the applications of fuzzy sets theory and artificial neural networks.

The FCM technique builds models in a form that can be easily understood (Papageorgiou \& Salmeron, 2013), learned and used by technical and nontechnical-experts alike 
(Alizadeh, 2018; Papageorgiou \& Salmeron, 2014). The flexibility of FCM in building, parameterizing, and modifying models that can perform within a relatively short period has increased its popularity during the past decade (van Vliet et al., 2010). It became an acceptable and widely used means for designing knowledge-based systems in various scientific areas (Nápoles et al., 2016; Jetter \& Kok, 2014). FCM has been used to support the decision-making process in complex planning (Jetter, 2006), such as planning information systems and product planning (Jetter \& Sperry, 2013 ). According to (Papageorgiou \& Salmeron, 2014), FCM is used in solving problems in various research fields such as medicine, control, business, robotics, environment, ecology, and safety research (Bevilacqua et al., 2013; Pourreza et al., 2018). The knowledge of complex matters can be captured in a standardized format and participatory technique involving academic experts or key industry actors and decision-makers such as managers, operators, etc. (Gray et al., 2012; Gray et al., 2014). However, more recently, the knowledge has been captured from secondary data sources, such as published research. For example, see (Alizadeh \& Jetter, 2017) and (Hajek et al., 2017).

The main goal of building FCMs around the problems of complex systems, mainly when the system's elements are in constant dynamic interactions, is that FCM can convert qualitative knowledge into quantitative and computable systems. Therefore, simulation based scenarios can be applied to predict and analyze the system's current and future state. Because FCM allows a wide range of scenarios to predict the behaviors and future state of the system it represents, and since it is a non-linear modeling technique and a proper means to model dynamic and complex matters in the data-poor environment, it is, therefore, an 
appropriate and promising methodology to model the phenomenon of organizational reliability. To understand how the FCM technique was invented and used, an overview of its theoretical background, cognitive maps, fuzzy set theory, and the artificial neural network will be discussed as follows.

\subsubsection{Theoretical Foundations}

FCM is a modeling methodology that originated in fuzzy logic and cognitive mapping to represent complex systems' knowledge under uncertainty based on neural network theory principles. An FCM is a signed directed graph that consists of nodes (concepts) that denote the system elements and links (edges) that connect the concepts with one another. The edges are directed to demonstrate the influence of the causally related concepts, marked with positive or negative signs to state the nature of the effect (i.e., increasing or decreasing), and weighted to specify the corresponding influence's strength. However, unlike neurons in an artificial neural network, where they are either ON (the activation level is 1 ) or OFF (the activation level is 0 or -1 ), FCM employs varying degrees of membership; that is why it is fuzzy. Because of this combined nature of the FCM, quantitative simulation can predict the system behaviors and state.

\subsubsection{Cognitive Maps}

A Cognitive Map (CM) is a graphical representation of qualitative knowledge that describes how a system works (Özesmi \& Özesmi, 2004). It is a digraph that has its root in graph theory and was first used by Robert Axelrod in 1976 to visualize social knowledge. Axelrod (1976) used cognitive mapping to represent the cause and effect relationships of political elites, captured in a participatory fashion by crowd people instead of researchers. 
Creating a cognitive map is the first step in building FCMs (Jetter \& Sperry, 2013 ). The modeler identifies the most significant variables (concepts) in the system and then draws signed arrows to connect the causally related concepts based on the provided reasoning. The required causal knowledge to build a cognitive map can be captured using various techniques such as interviews, focus groups, workshops, mapping exercises, and qualitative analysis of textual data.

Figure 4 below depicts a simple causal cognitive map that consists of four concepts and five causal links. In this map, concept A increases concept B, C, and D, yet increasing the value of concept A may or may not increase concept D in the system's steady state. That is because concept $\mathrm{D}$ is a driven variable that depends not only on the positive impacts of concepts $\mathrm{A}$ and $\mathrm{C}$ but also on the negative influence of concept $\mathrm{B}$. Therefore, the final value of concept $\mathrm{D}$ is contingent on the strength of all the positive and negative causal links. However, cognitive maps in the earlier introduction and applications were binary. In other words, the strength of the causal relationships was either $(+1)$ for all the positive links or (-1) for all the negative connections.

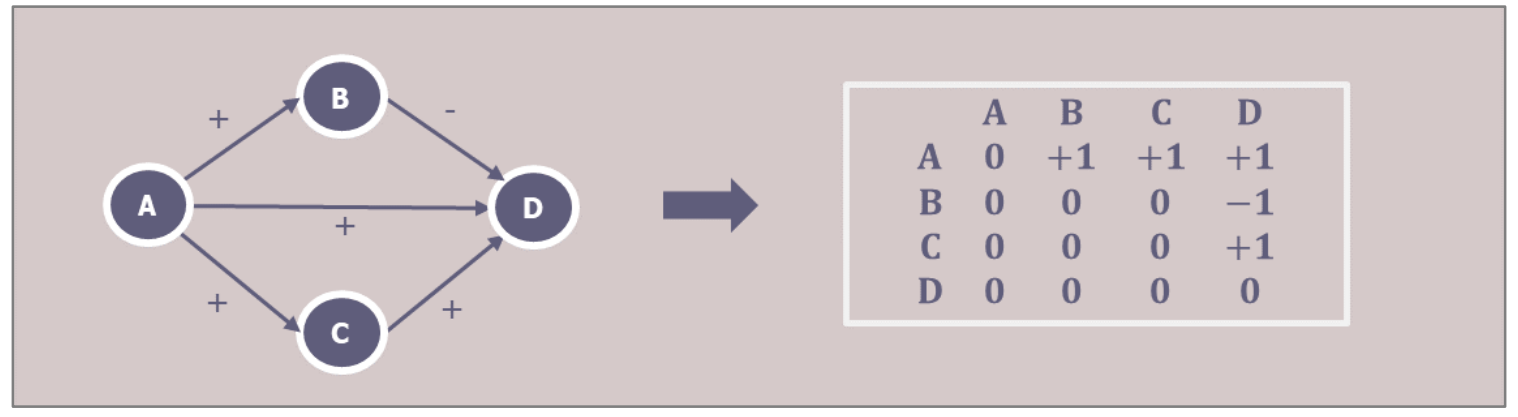

Figure 4: Simple Causal Cognitive Map and the Corresponding Adjacency Matrix

As a digraph, a cognitive map can be turned into a square $(n \times n)$ adjacency matrix, where $n$ is the number of the concepts in the map. This matrix indicates whether pairs of concepts 
are adjacent or not in the graph, and it demonstrates the causal relationships among the adjacent pairs in a mathematical format. Each element of the matrix $\left(\mathrm{m}_{\mathrm{ij}}\right)$ is a value function of the adjacent pair of concepts (i.e., $\mathrm{m}_{\mathrm{ij}}=f\left(\mathrm{C}_{\mathrm{i}}, \mathrm{C}_{\mathrm{j}}\right)$ ). If $\mathrm{C}_{\mathrm{i}}$ causally increases $\mathrm{C}_{\mathrm{j}}, \mathrm{m}_{\mathrm{ij}}$ to $(+1)$, and if decreases $C_{j}, m_{i j}$ to $(-1)$, then when there is no link, $m_{i j}$ is $(0)$. The corresponding adjacency matrix is shown in Figure 4 above. Since all the concepts in this example have no feedback or influence on themselves, their values $\left(\mathrm{m}_{11}, \mathrm{~m}_{22}, \mathrm{~m}_{33}, \mathrm{~m}_{44}\right)$ in the matrix were set to zero.

\subsubsection{Fuzzy Set Theory}

Modeling complex systems to represent real situations often encounter uncertain and vague information resulting from human subjectivity and imprecision. Uncertain information can be partially precise, which is usually handled using probability theory and statistics, or imprecise, which can be handled using fuzzy set theory to provide measurable precisions for verbal statements (Kangari \& Boyer, 1989). Real situations often are not crisp (i.e., fuzzy), and therefore, they cannot be described precisely (Zimmerman, 2001).

Zadeh (1965) proposed the fuzzy set theory based on the axiomatic logic considering human subjectivity and imprecision (Kangari \& Boyer, 1989). It is an extension of the classic theory of sets (Jetter, 2006) and a means to describe ambiguous situations by using a more precise numerical context (Zimmerman, 2001). The difference between the classic theory of set and fuzzy set theory is that the former considers an object in a binary association with a set (i.e., the object either belongs to the set or not). For example, a 29years-old man is not a middle-aged group member if the group boundaries are between 30 and 50 years old. These crisp boundaries do not match human precision because people 
define age boundaries differently. However, the latter (fuzzy set theory) considers an object as a member of a set with a varying degree of membership (i.e., partial belongingness), which is usually represented in the interval [0;1]. A fuzzy set can expand the boundaries of the above example from crisp values to a range that includes the 29 -years-old man as a partial member of this group with a specific degree of belongingness. Based on this conception, Kosko (1986) integrated fuzzy logic into cognitive mapping and introduced fuzzy cognitive maps. In FCMs, concepts' values are not only $0,+1$, and -1 , but also values within the intervals $[0 ; 1]$ and $[-1 ;+1]$. Also, the strengths of causal links are not in a binary fashion (i.e., +1 or -1 ) as with cognitive maps, but use values in the range of $[-1 ; 1]$. Figure 5 below is an FCM with weighted edges based on fuzzy logic and the map's corresponding adjacency matrix.

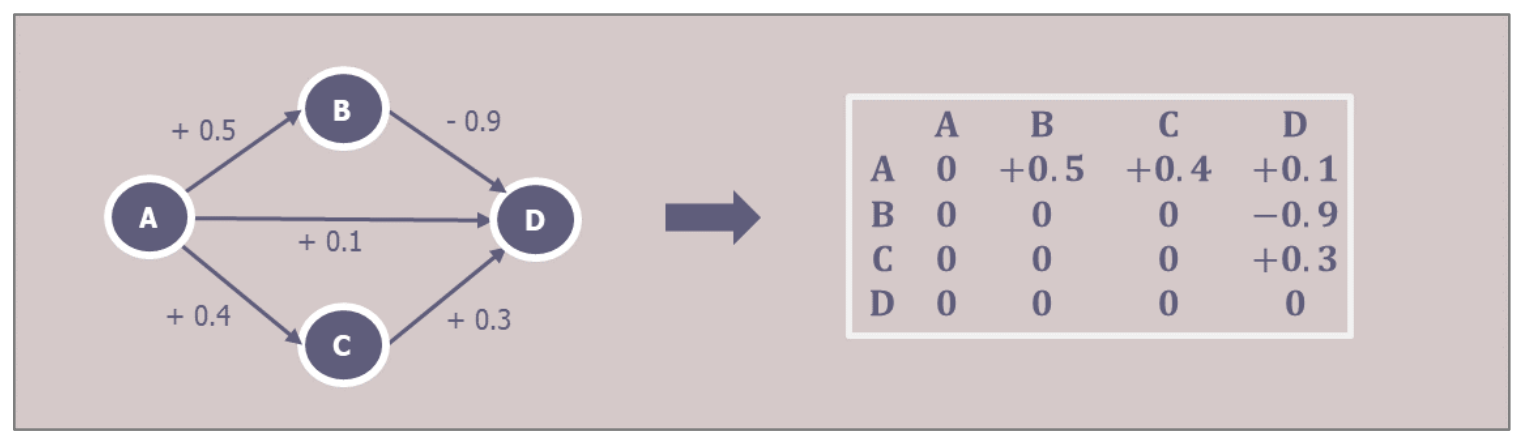

Figure 5: Simple FCM and the Corresponding Adjacency Matrix

The value of each concept in the map is calculated based on the values of all the influencing concepts multiplied by the strengths of the causal links using equation (1) (Alizadeh, 2018):

$$
C_{i}^{(k)}=C_{i}^{(k-1)}+\sum_{A l l j \neq i} C_{j}^{(k-1)} W_{j i}
$$

Where $W j i$ is the strength of the causal link between concepts $C i$ and $C j$ at iteration $k$. 
To examine how a particular scenario can change the value of the concepts in an FCM, a state vector $(1 \times n)$, where $n$ is the number of the map's concepts, is multiplied by the corresponding adjacency matrix, by which a new state vector would result. This process is repeated until the system reaches a study state or new iterations lead to the same outcomes. For instance, activating concept $\mathrm{A}$ in Figure 5 above (i.e., increasing its value from 0 to 1 ), while other concepts are turned off (i.e., set their values to 0), the initial state vector (Iteration 1) is as follows:

$S_{1}^{T}=\left[\begin{array}{llll}1 & 0 & 0 & 0\end{array}\right]$

This first state vector is multiplied by the adjacency matrix (Iteration 2):

$\left[\begin{array}{llll}1 & 0 & 0 & 0\end{array}\right] \times\left[\begin{array}{cccc}0 & 0.5 & 0.4 & 0.1 \\ 0 & 0 & 0 & -0.9 \\ 0 & 0 & 0 & 0.3 \\ 0 & 0 & 0 & 0\end{array}\right]=\left[\begin{array}{llll}0 & 0.5 & 0.4 & 0.1\end{array}\right]$

The new state vector; $S_{2}^{T}=\left[\begin{array}{llll}0 & 0.5 & 0.4 & 0.1\end{array}\right]$

The second state vector is multiplied by the adjacency matrix (Iteration 3):

$\left[\begin{array}{llll}0 & 0.5 & 0.4 & 0.1\end{array}\right] \times\left[\begin{array}{cccc}0 & 0.5 & 0.4 & 0.1 \\ 0 & 0 & 0 & -0.9 \\ 0 & 0 & 0 & 0.3 \\ 0 & 0 & 0 & 0\end{array}\right]=\left[\begin{array}{cccc}0 & 0 & 0 & -0.33\end{array}\right]$

The new state vector; $S_{3}^{T}=\left[\begin{array}{llll}0 & 0 & 0 & -0.33\end{array}\right]$

The third state vector is multiplied by the adjacency matrix (Iteration 4):

$\left[\begin{array}{llll}0 & 0 & 0 & -0.33\end{array}\right] \times\left[\begin{array}{cccc}0 & 0.5 & 0.4 & 0.1 \\ 0 & 0 & 0 & -0.9 \\ 0 & 0 & 0 & 0.3 \\ 0 & 0 & 0 & 0\end{array}\right]=\left[\begin{array}{llll}0 & 0 & 0 & 0\end{array}\right]$ 


\subsubsection{Integrating Artificial Neural Network Theory in FCMs}

Artificial Neural Network (ANN) is a modeling technique that has been developed based on the mathematical representations of the biological nervous systems (Abraham, 2005). This technique emerged after the introduction of basic neurons by McCulloch and Pitts in 1943 (Alizadeh, 2018). In ANN, neurons receive stimuli (input signals with different strengths), process, and transmit them to the adjacent neurons. When the received signals are strong enough, they can be transmitted to all the network neurons (Jetter, 2006). The connection strengths weigh the effects of the transmitted signals and transform them through a transferring function that displays the neurons' nonlinear characteristics (Abraham, 2005). Figure 6 below illustrates this process graphically, and the output signal $O$ of the neuron can be calculated using equation (2):

$$
O=f(n e t)=f\left(\sum_{j=1}^{n} w_{j} x_{j}\right)
$$

Where $w_{j}$ is the weight vector, and $f(n e t)$ is the transfer function, while the variable net is a product of the weight and input vectors:

$$
n e t=w^{T} x=w_{1} x_{1}+\cdots+w_{n} x_{n}
$$

Where $T$ is the transpose of the matrix.

The output value $O$ is computed as follows:

$$
O=f(\text { net })= \begin{cases}1 & \text { if } w^{T} x \geq \theta \\ 0 & \text { if otherwise }\end{cases}
$$

Where $\theta$ is the threshold level. 


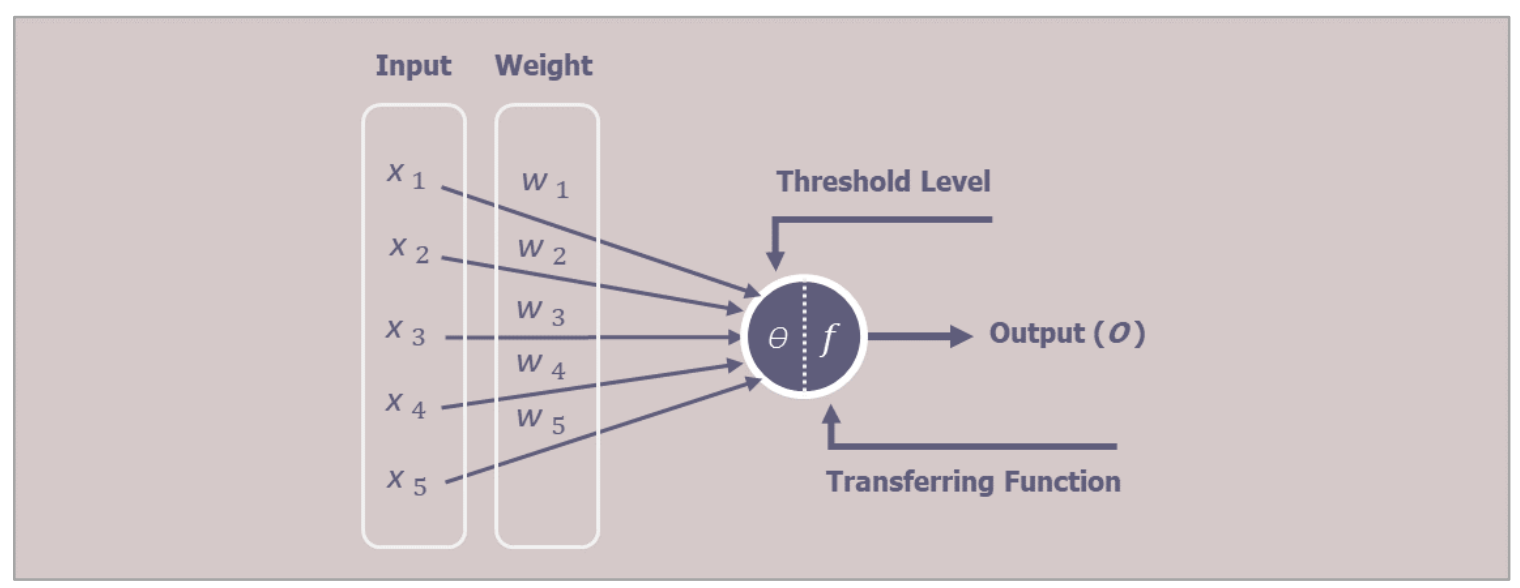

Figure 6: Signal Processing in an Artificial Network

Several types of inference (activation) functions can be used in FCM modeling techniques such as binary, linear, sigmoid, and hyperbolic tangent. Modelers usually choose what fits their modeling objectives and results presentations (Alibage et al., 2018).

Binary function:

$$
f(x)= \begin{cases}-1 & \text { for } x<0 \\ 0 & \text { for } x=0 \\ 1 & \text { for } x>0\end{cases}
$$

Linear function:

$$
f(x)=\left\{\begin{array}{lr}
-1 & \text { for } x \leq-1 \\
x & \text { for }-1<x<1 \\
1 & \text { for } x \geq 1
\end{array}\right.
$$

The sigmoid function produces outputs between $[0,1]$ for any input variable.

$$
f(x)=\frac{1}{1+e^{-\lambda x}}
$$

The hyperbolic tangent function produces outputs between $[1,-1]$ for any input variable.

$$
f(x)=\tanh \lambda x \mathrm{~W}
$$


Where $\lambda$ is a parameter used to determine the transfer function's appropriate shape, whereby modelers specify the function slope or the degree of fuzzification.

Kosko $(1988 ; 1991)$ integrated the principles of ANN theory in the FCMs, which turned them into recursive artificial neural networks. ANN application has enabled the FCM technique to conduct various scenarios regarding complex systems' dynamic behaviors and predict system states. Applying ANN to FCMs has solved the problem of misidentifying which system's elements can stay active in the short term when activating a particular concept(s) and which ones last under the activation effects for a more extended period (Jetter, 2006). The use of sigmoid and the hyperbolic tangent functions to examine the performance of the FCM shown in Figure 5 above yielded the results shown in Table 5 and Table 6 and presented in Figure 7 and Figure 8, respectively.

Table 5: Results of Using Sigmoid Function

\begin{tabular}{|c|c|c|c|c|}
\hline Iteration & $\boldsymbol{A}$ & $\boldsymbol{B}$ & $\boldsymbol{C}$ & $\boldsymbol{D}$ \\
\hline 1 & 1 & 0 & 0 & 0 \\
\hline 2 & 0.5 & 0.6225 & 0.5987 & 0.525 \\
\hline 3 & 0.5 & 0.5622 & 0.5498 & 0.4181 \\
\hline 4 & 0.5 & 0.5622 & 0.5498 & 0.4278 \\
\hline
\end{tabular}

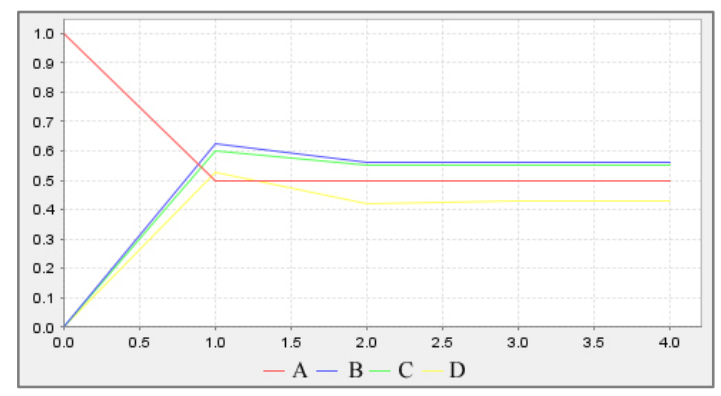

Figure 7: Effects of Sigmoid Function 
Table 6: Results of Using Hyperbolic Tangent Function

\begin{tabular}{|c|c|c|c|c|}
\hline Iteration & $\boldsymbol{A}$ & $\boldsymbol{B}$ & $\boldsymbol{C}$ & $\boldsymbol{D}$ \\
\hline 1 & 1 & 0 & 0 & 0 \\
\hline 2 & 0 & 0.4621 & 0.3799 & 0.0997 \\
\hline 3 & 0 & 0 & 0 & -0.2931 \\
\hline 4 & 0 & 0 & 0 & 0 \\
\hline
\end{tabular}

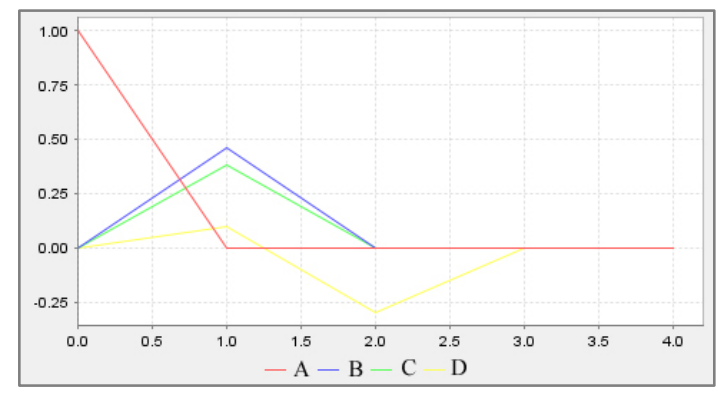

Figure 8: Effects of Hyperbolic Tangent Function

\subsection{Thematic Analysis (TA)}

Thematic Analysis (TA) is a qualitative research method that identifies and interprets latent or hidden patterns of meaning across qualitative data under study (Clarke \& Braun, 2014). Although this method has been known within the literature on qualitative research since 1979 (Braun \& Clarke, 2006; 2014), its guidelines were first put into place in the late of 1990s by Boyatzis (1998) to describe the development of the codes and themes, where the method sought independence from grounded theory. Nonetheless, TA is still considered a grounded theory but within a theoretical framework (Clarke, 2017), and because there is no clear distinction between qualitative content analysis and thematic analysis (Sandelowski \& Leeman, 2012), the two methods are often used interchangeably (Braun \& Clarke, 2006; Clarke, 2017). 
TA became popular over the last decade and has been widely used in qualitative research (Clarke, 2017; Gross, 2013; Guest et al., 2014), particularly within social and health sciences, following a widely cited research paper by Braun \& Clarke (2006). TA can be used to analyze most qualitative data types, including interviews, focus groups, qualitative surveys, story completion tasks, diaries, vignettes, and a wide range of secondary sources, including printed materials, online and electronic materials, and broadcast media and films. This method moves beyond explicit words or phrases and focuses on identifying and describing both implicit and explicit ideas within the data, or in other words, "themes" (Guest et al., 2014).

The purpose of using TA is to facilitate the categorization process of the data and the construction of the explicit and implicit various meanings and descriptions of all the relevant concepts that emerge from the literature, including the concepts that support as well as those that preclude the creation of a complex phenomenon, which in the case of this research is organizational reliability. This method provides a beneficial qualitative approach, particularly for conducting applied research such as health, policy, or practice fields (Braun \& Clarke, 2014), education, early childhood, and health and safety research (Salleh et al., 2017; Lekka \& Sugden, 2011).

\subsubsection{Theme Definition and Development}

A theme is defined as "an extended phrase or sentence that identifies what a unit of data is about and/or what it means" (Saldaňa, 2016, p. 199), and generally known to meaningfully capture the most significant data or information regarding the research objectives, and to characterize some level of patterned meanings (Braun \& Clarke, 2006). 
In other words, a theme identifies an area of data that tells the readers something about it (Sandelowski \& Leeman, 2012).

Themes are developed as a researcher assigns qualitative $\operatorname{codes}^{12}$ to the text and tries to organize and categorize the codes into larger patterns. Themes can emerge from conceptual codes and sub-codes and relationship codes, which label data that link concepts to each other (Bradley et al., 2007).

Moreover, categorizing the coded text into categories and sub-categories is, in fact, comparing them based on their "particular-reality" while theming is converting them into a "general-abstract" meaning (Saldana, 2016) as illustrated in Figure 9. For example, theming two or more categories of technical staff that have been categorized based on their specific technical disciplines (i.e., their "particular-reality," such as mechanics, electricians, etc.) as "technicians" is converting their specialties into a "general-abstract" description. This process reduces complexities in the text and provides the readers with bigger pictures of the hidden patterns for clarity and understanding; however, nuanced details remain in the themes' definitions.

Figure 9 demonstrates three paths to develop themes from textual data over streamlined codes. In path 1 , a theme or sub-theme is developed by grouping two or more categories of codes if the categories are: (1) Sharing similar or general concepts such as theming various categories of smokers into a theme or sub-theme of "people with high-risk cancer,"

\footnotetext{
${ }^{12}$ A code in qualitative research refers to "the most basic segment, or element, of the raw data or information that can be assessed in a meaningful way regarding the phenomenon" (Boyatzis, 1998, p. 63). More discussions on qualitative codes provided in the next chapter (Chapter 4).
} 
or (2) leading to (causing) the same or a similar outcome such as theming various categories of errors into a theme or a sub-theme of "operational failure." In path 2, a theme is developed by converting a unique category of codes into a theme or a sub-theme, such as theming specific kinds of drugs under the theme or sub-theme of "specific treatment." In path 3 , the code is unique and conveys a distinctive meaning that cannot be grouped with other codes into one category or sub-theme; thus, it is converted into a theme or a subtheme, such as in the case of coding a medicine student in a piece of text that mainly focused on STEM students.

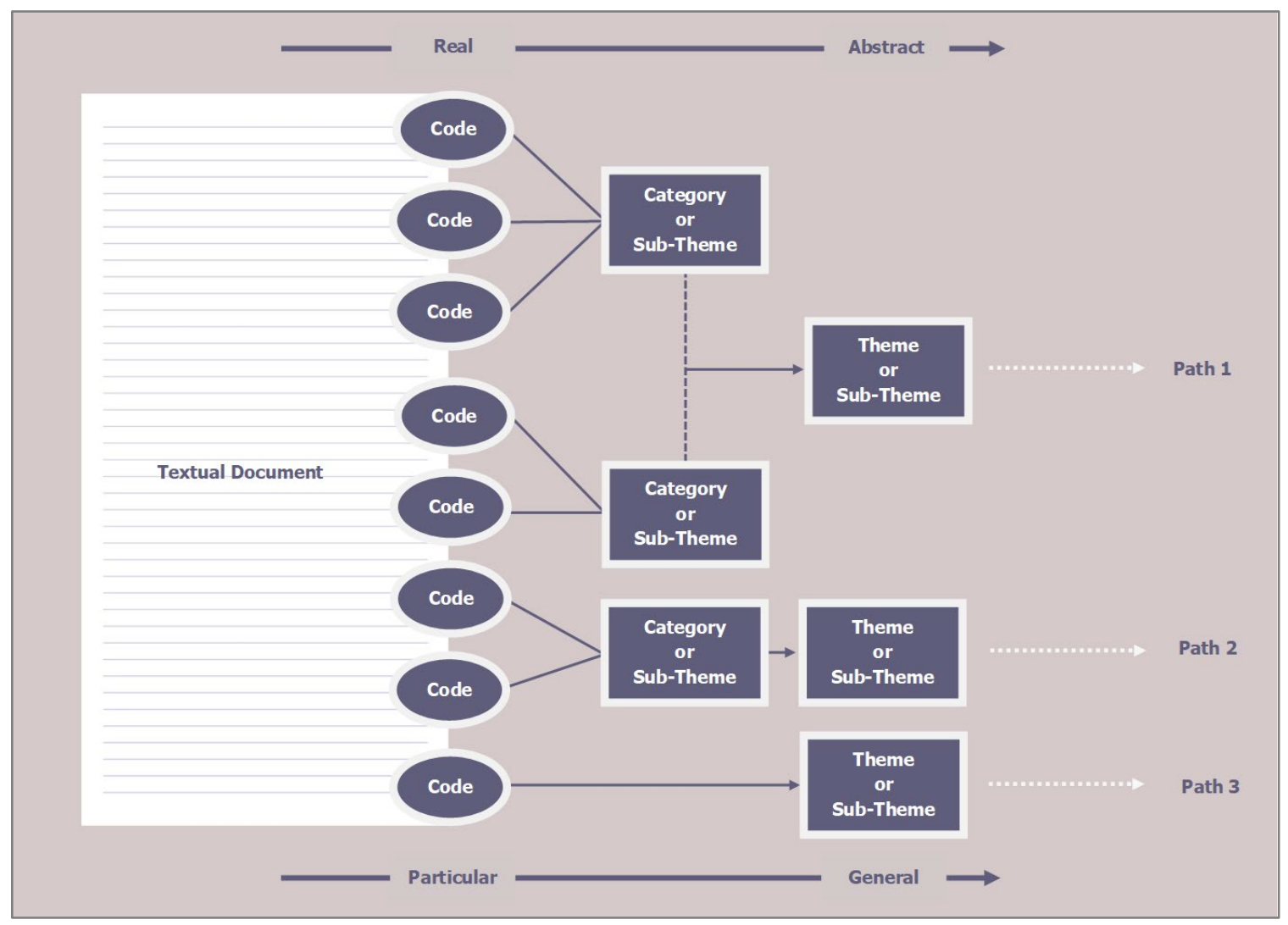

Figure 9: Various Paths to Develop Themes from Textual Data 
According to Clarke (2017), there are two types of themes: Bucket themes and storybook themes. A bucket theme (also referred to as a domain summary) is a combination of different meanings, such as risk and benefits, which means that there is no sense of an integrated or a shared meaning other than a summary of everything said concerning a specific topic in such a theme. However, a storybook theme is conceptualized as a pattern in the data supported by a central concept that organizes the analytic observations.

\subsubsection{Phases of Thematic Analysis}

TA is a structured method and follows a detailed and lengthy process that consists of the following principles, as suggested by Braun \& Clarke (2006). Extensive discussions around these principles are provided in Chapter 4.

Phase 1: Understanding the Data: During this phase, the researcher develops a broad understanding of and familiarizes himself/herself with the data set. This is especially true when the data were not collected by the same researcher, such as analyzing the literature around a phenomenon.

Phase 2: Generating Initial Codes: This phase involves developing a preliminary set of codes from the data as depicted and explained in Figure 9. These codes categorize a feature of the data (semantic content or latent) that appears significant to the analysis. The coding process, including the coding strategy, procedure, and coding reliability, are discussed extensively in Chapter 4.

Phase 3: Searching for Themes: During this phase, the analysis's focus takes a broader level of themes based on the developed set of codes. It involves categorizing the codes into 
themes based on their general meaning and similarities. The process of theming, including the theming strategy and procedure, is also discussed extensively in Chapter 4.

Phase 4: Reviewing Themes: This phase involves a more in-depth examination of the initial themes, whereby a preliminary theme can be merged into other themes when it doesn't have enough data to stand on its own, or it can be collapsed into two or more themes when it consists of diverse data.

Phase 5: Defining and Labeling Themes: Through this phase, the core of what each theme is about is identified, and the characteristic of the data that each theme captures is determined.

Phase 6: Producing the Report: In this phase, the objective is to produce a report that speaks a complete story about the data.

\subsection{Thematic Network}

A thematic network (TN) is an "analytic tool draws on core features that are common to many approaches in qualitative analysis" (Attride-Stirling, 2001, p. 386). A TN (also known as a thematic map) depicts and defines the causal relationships between themes at different levels (Braun \& Clarke, 2006; Guest et al., 2014). Building a thematic network based on published research reveals notable themes at different levels and enables their structuring and representation. This thematic network provides the so-called "web-like" network that summarizes the main themes or patterns constituting a piece of text (AttrideStirling, 2001). 
In Figure 10, a thematic network arranges the abstraction of themes at three levels: Basic (lower-order) themes, organizing (middle-order) themes, and global (super-ordinate) themes, which each of which will be discussed as follows.

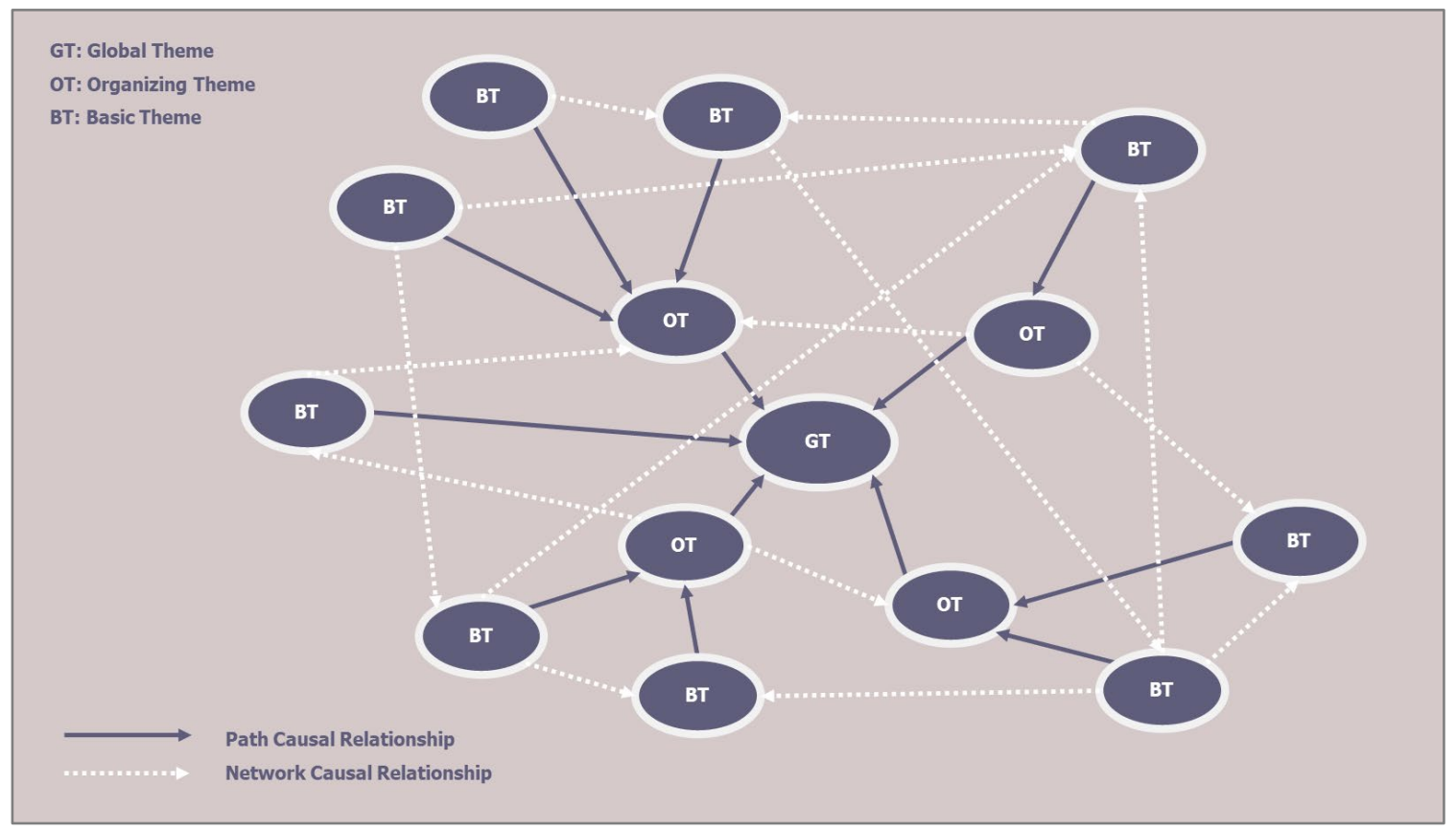

Figure 10: Thematic Network (Global Theme)

However, this three-level arrangement does not necessarily arrange themes in a hierarchical order. It is still web representations or an organizing path regarding the cause and effect relationships between each two causally related themes. In path causal relationships, a global theme is a bucket or storytelling theme of various organizing themes, where each of which is a bucket or storytelling themes of basic themes.

In this network, themes on different levels can also be in causal relationships with one another. Constructing a thematic network is also a structured process that typically follows similar phases in thematic analysis or complementing them. The three-levels themes suggested by Attride-Stirling (2001) are defined and described as follows: 
Basic Themes: A basic theme is a lowest-order theme that is elicited directly from the textual data to convey a statement of understanding around a central idea that leads to the creation of organizing and global themes. Basic themes usually provide simple meanings of the data under study. Therefore, they do not say much about it unless they stand enough on their own to reflect the exact meaning or provide meanings in the context of other basic themes to the higher-level themes.

Organizing Themes: Organizing themes are middle-order themes that organize basic themes into groups of similar issues. They are clusters of meanings that summarize groups of basic themes. Therefore, they are more abstract and more revealing of what is going on in the texts. Organizing themes play the role of enhancing the meaning and importance of broader themes and demonstrating their main assumptions. A group of organizing themes, therefore, constitutes a global theme.

Global Themes: Global themes are super-ordinate themes that encompass the principal metaphors in the data. They are the concluding remarks concerning a phenomenon or significant stories in the analyzed data. Global themes are groups of organizing themes that together introduce arguments or demonstrate given issues or reality. They make sense of groups of lower-order themes that are distracted from and supported by the data. Thus, global themes speak what the texts are about within the context of a given analysis, considering that when the analyzed text is complex and constitutes various ideas, it can yield more than one global theme. However, global themes are usually less in number than organizing and basic themes. They form networks of causally related themes so that 
analyzing a text that yields more than one global theme can result in more than one thematic network.

\subsection{Thematic Proximity}

Thematic proximity is a relational content analysis approach to identify implicit patterns among the text's descriptive units under study (Armborst, 2017). Although it is not an explicitly mixed method that necessitates collecting and analyzing qualitative and quantitative data, thematic proximity enables various statistical coefficients, a numerical interpretation for the interrelated qualitative statements. Therefore, it provides a means to quantify the strength of the causal relationship between the causally related unit (i.e., codes, themes, patterns, etc.).

Using this approach aims to reduce or provide an alternative to the total reliance on subject experts in assigning weights for the edges of the causally descriptive models. As discussed before, assigning weights for these relationships through experts is often challenging and does not usually result in precise weights. Thematic proximity can be measured using various quantitative techniques, yet each technique's appropriateness depends on the research context and methodology. In the text mining approaches, Cosine Similarity Index, which is a vector-based approach, is often used to compares two texts in terms of shared words (Oleinik, 2011; Salton, 1983).

This technique produces a score between [0] when there is nothing in common and [1] when the two texts are identical. Cosine similarity can be calculated using equation (9) below. It measures the cosine of the angle between the two vectors of words, where the vectors that do not share any content are at a perfect 90-degree angle from one another, 
resulting in a score of [0]. In contrast, vectors that share identical content have zero degrees between them, resulting in a score of [1] (Acree et al., 2016).

$$
\cos \theta=\frac{A . B}{\|A\| \cdot\|B\|}=\frac{\sum_{i=1}^{n} A_{i} \cdot B_{i}}{\sqrt{\sum_{i=1}^{n} A_{i}^{2}} \cdot \sqrt{\sum_{i=1}^{n} B_{i}^{2}}}
$$

Although this approach is simple to implement and interpret, it has some downsides. First, similarity may not necessarily reflect proximity or correlation between two compared sets of texts. Second, since this technique placed in the broader family of text analysis techniques that are known as "bag of words" (Acree et al., 2016), it does not take word order or context into consideration when comparing two texts (sentence, paragraph, or the whole set of text). Like a bag of words, this measure depends on the frequency of words in each text, where the results would be significantly changed when the frequency of one word (i.e., the number of times a word is mentioned) in each of the compared text is increased or decreased.

Figure 11 illustrates how text vectors are projected on each other to form an angle that leads to determining the value of the Cosine similarity index. In this figure, only two words (word 1 and word 2) were considered to simplify this technique's overall configuration, which clearly shows that the vectors will become narrow or pull away depending on increasing or decreasing the number of one word in each set of text. In contrast, the other word's number remains the same or changes inversely, thus increasing or decreasing the angle. 


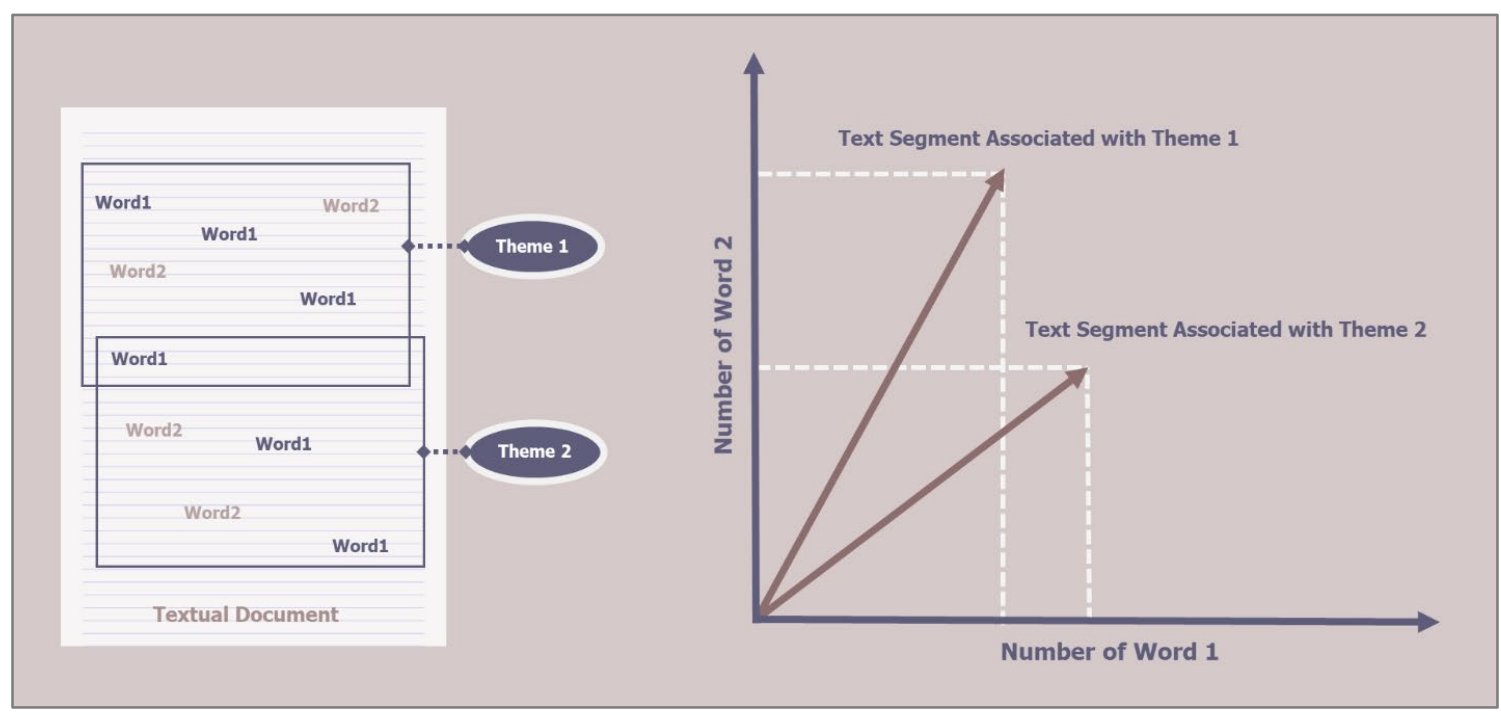

Figure 11: Text Vectors Projection in Cosine Similarity Index

Another approach to estimating thematic proximity between interrelated themes uses the Jaccard Similarity Coefficient (c-coefficient) to analyze the frequency of the cooccurrences or overlap of descriptive units (i.e., codes, themes, patterns, or categories) within the entire set of data under analysis. The c-coefficient is integrated into most of the qualitative data computer-assisted software such as Atlas.ti, NVivo, QDA Miner, and MAXQDA, and it is mostly used in content analysis and other qualitative methods. It calculates the strength of the relationship between two units similar to a correlation coefficient in a range between [0] for no correlation and [1] for maximum correlation; however, no significance is provided (Friese, 2018). The c-coefficient is calculated using the following equation (10), where $n_{1}$ is the number of occurrences (grounding) of unit 1 in the whole coded text, $n_{2}$ is the number of occurrences of unit 2 , and $n_{12}$ is the number of co-occurrences of both codes.

$$
c=\frac{n_{12}}{\left(n_{1}+n_{2}\right)-n_{12}}
$$


Importantly, there are inherent issues with using the c-coefficient to provide accurate measures due to some deficiencies in embedding this tool into a more logical algorithm in qualitative software such as Atlas.ti. Even when there is a clear indication of a strong relationship between two analytical units (themes), the c-coefficient may underestimate this relationship's strength, particularly when one unit is attached more often to the text segments than the other related unit. For example, if $n_{1}=100, n_{2}=10$, and $n_{12}=5, c=$ 0.048. Furthermore, the c-coefficient does not consider the proportion of overlapping content in determining how strong the relationship is (Friese, 2018). Figure 12 depicts two cases of how c-coefficient is calculated and why it does not often provide an accurate measure.

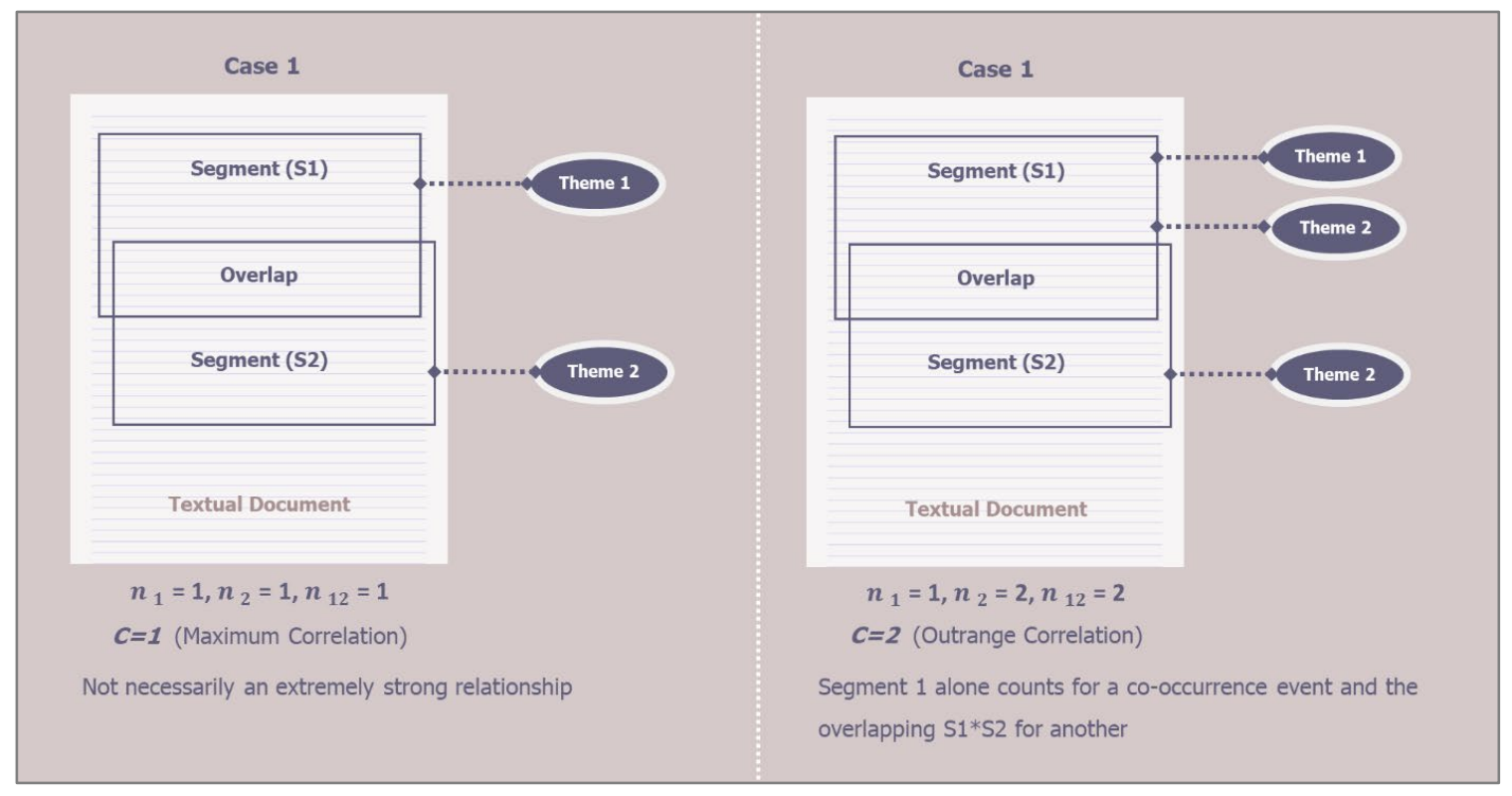

Figure 12: Possible Cases Resulted from The Use of c-Coefficient

Most recently, Armborst (2017) proposed a new statistical technique to estimate the thematic proximity between causal descriptive units (or themes) using the so-called thematic coefficient (t-coefficient). This method overcomes the discussed limitations using 
the other measures (i.e., c-coefficient and Cosine index) by considering the proportions of text intersections and word frequencies instead of the frequency of code co-occurrences. The t-coefficient estimates proximities based on the text content that every two related units share. The more content they share, the stronger the relationship between them. This coefficient is calculated using the following equation.

$$
t=\frac{1}{2}\left(\frac{n_{12}}{n_{1}}+\frac{n_{12}}{n_{2}}\right)
$$

Where $n_{1}$ is the total number of words classified with unit 1 (theme 1), $n_{2}$ is the total number of words classified with unit 2 (theme 2), and $n_{12}$ is the number of the shared words between the two units. A graphical representation of how the variable of t-coefficient is identified is in Figure 13.

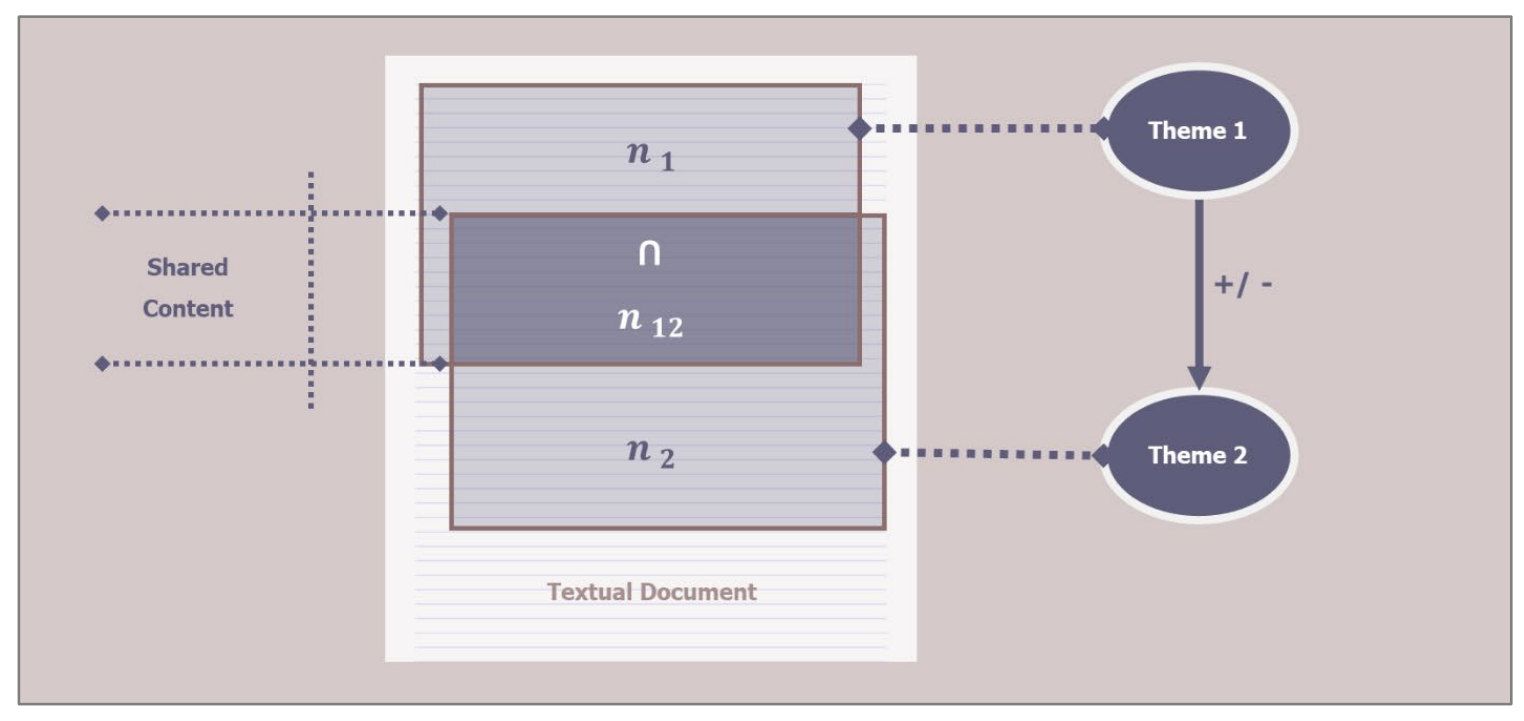

Figure 13: Graphical Representation of How the Variable of t-coefficient Identified

The variable explained in equation (11) can be calculated by generating word lists associated with each theme using the retrieval tools or word lists provided by computer 
software such as Atlas.ti. The results are values in the intervals of $[0,1]$, where $[0]$ indicates no correlation, and [1] indicates a maximum relationship.

Notably, the t-coefficient only yields positive values; however, the nature of the causal relationships (i.e., positive or negative) is defined by the researcher(s) and decided by the text's interpretation and analysis. Furthermore, to compare the calculated values of the tcoefficient for all the connections in the thematic network(s), both of cosine similarity index and c-coefficient will be calculated and compared. More details and numeric comparisons will be provided in Chapter 5 .

\subsection{Exploratory Modelling and Analysis (EMA)}

In traditional modeling approaches such as predictive modeling, the model is developed based on known facts and sufficient knowledge to accurately reflect the modeler's understanding of how a system works and then predicts the model's behaviors based on inputs (Bankes, 1992; 1993). Such models can be verified when the systems they represent are visible and quantifiable, their causal structure does not change over time, and the phenomena gathers enough information (Hodges \& Dewar, 1992). However, when modeling a complex system where the model structure (i.e., how the model is built) is based on implicit and explicit assumptions, inputs to the model (policy and decision-making processes) are expected to encounter various uncertainties. Therefore, modeling such systems using traditional approaches may not replicate a reliable image of how the system works and behaves in the real world nor predict outcomes for the given inputs.

To overcome potential uncertainties in modeling complex systems, including those that are modeled as FCMs, several research fields such as economics and environmental science 
have shifted away from predictive to explorative modeling, by which the model can be simulated concerning a wide range of various inputs (Kwakkel \& Pruyt, 2013). A capable approach to model-based decision support with a high level of uncertainty is Exploratory Modelling and Analysis (EMA), a quantitative technique that uses computational experiments to explore the implications of defined assumptions and hypotheses (Bankes, 1993; Agusdinata, 2008).

The RAND Corporation has initially developed EMA to provide decision support in situations when various types of uncertainties (i.e., parametric, structural, and methodological) cannot be simplified or reduced (Bankes, 1992). It is a suitable technique to simulate explorative models, particularly when critical data does not create a single model that articulates how the system works in real-world settings (Kwakkel \& Pruyt, 2013; Kwakkel et al., 2010). However, the use of EMA neither reduces nor eliminates uncertainties around a specific problem nor simplifies it. Instead, EMA provides computer decision support for policy analysis under the same level of complexity and uncertainty (Bankes, 1992). For example, EMA can answer questions such as, under which conditions can a particular policy do well? (Kwakkel \& Pruyt, 2013). Furthermore, EMA is not a modeling technique per se, but it is an approach to the modeling and the simulation processes (Alizadeh, 2018).

In this research, the introduced Fuzzy Cognitive Mapping (FCM) is the modeling methodology that is used within the framework of EMA. Figure 14 compares and explains how EMA results in different outputs based on various and different inputs. In the case of exploratory modeling, the edge weight is a range or interval that yields different outcomes, 
through which the decision-makers can understand under which conditions the policy, intervention, and so forth perform better. The use of exploratory modeling will allow simulating a range of various measures that vary between "very weak" to "very strong," thus effectively not only simulating outcomes in one FCM model but in multiple FCM models (Alizadeh, 2018).

Kwakkel et al. (2010, p. 5) suggest basic steps in EMA: (1) to develop a fast and simple model of the system of interest; (2) to generate an ensemble of future worlds; (3) to specify a variety of policy options; (4) to calculate and compare the performance of the various options across the ensemble of future worlds, and; (5) to iterate through steps until a satisfying policy option emerges. 


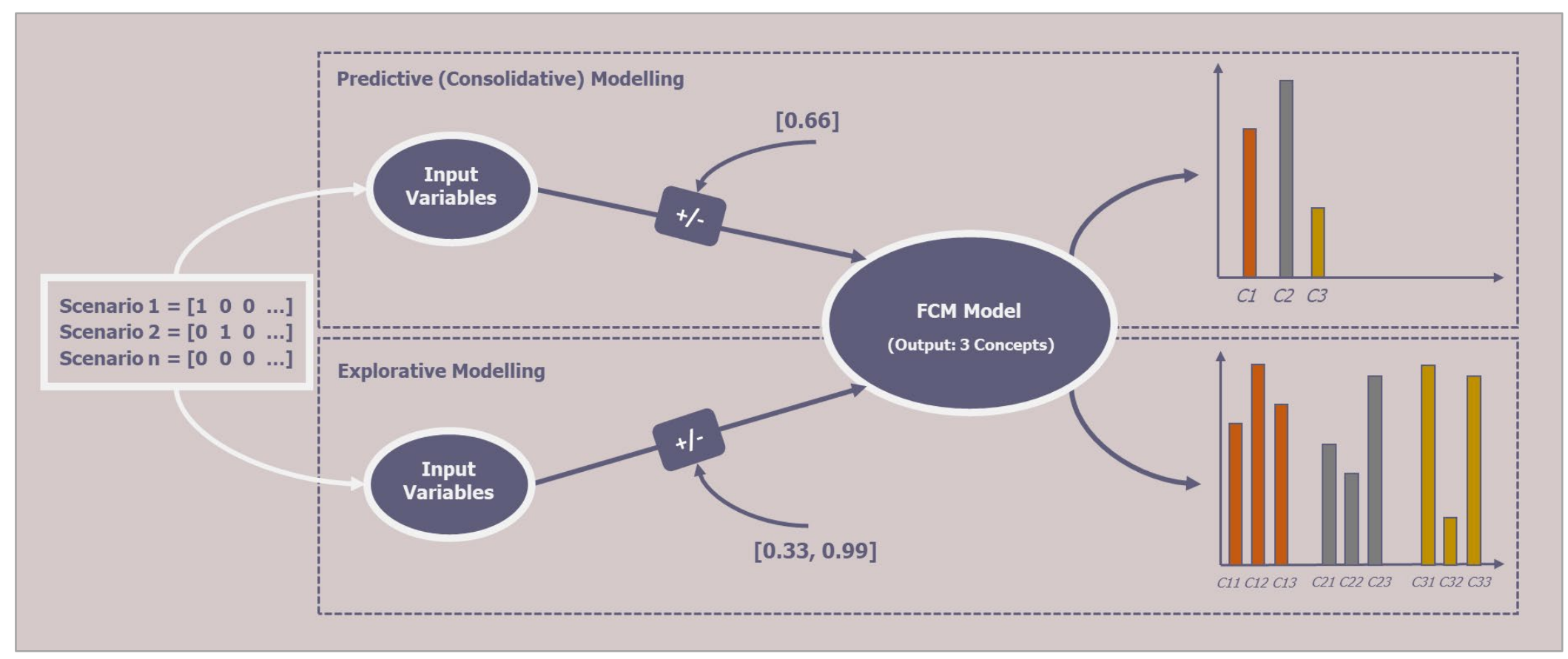

Figure 14: Graphical Representation of Explorative VS. Traditional FCM Modeling 


\subsection{Model Testing for Verification}

In qualitative research, the quality of research outcomes is frequently evaluated based on the rigor with which the research was produced. According to Morse et al. (2002) and Saunders et al. (2015), rigor encompasses research credibility, authenticity, suitability, relevance, transferability, and objectivity. For modeling studies, Sterman similarly recommends establishing criteria for model quality from the beginning of the research process to prevent it from resulting in a useless model (Sterman, 2000). Accordingly, this section develops plans for model testing and verification. A model is useful only when it resembles the real-world system it represents, which requires testing it against what is know about the real-world system (Sterman, 2000). Model verification is a structural process that establishes confidence around the model regarding its purposes and usefulness instead of declaring a model to be "correct" and approve or disapprove it (Forrester \& Senge, 1980). According to Barlas (1996), the process of model testing depends on the model type, which he classifies into two types; (1) correlational, or purely data-driven model, and (2) causal descriptive or theory-driven model that describes how real-system functions. While a correlational model can pass the tests when its behaviors resemble the behaviors of the real-system it represents, a causal-descriptive model can pass the tests only when both its structure and behaviors match the structure and behaviors of the real system. Barlas (1996) states that all design-oriented models, including system dynamics, are causal-descriptive and must pass various tests to be verified. FCM models are causaldescriptive models that represent how systems function in the real world. Accordingly, they also need to undergo model verification. 
The literature on testing and verifying FCM models recommends to borrow and modify strategies and techniques for model verification used in system dynamics such as expected behavior, extreme conditions, and sensitivity analysis (Jetter \& Kok, 2014). This research does the same and borrows aspects of formal model testing suggested by Forrester $\&$ Senge (1980) and Barlas (1996), as well as tests for assessment of dynamic models proposed by Sterman (2000). The process of model testing is graphically illustrated in Figure 15 below. The arrows in blue refer to the process direction which flows from testing the model structure first then the model behaviors as Barlas (1996) suggests, while the dotted arrows in orange refer to the process feedback, which allows modifying the structure of the core model whenever the model does not pass any of the selected tests.

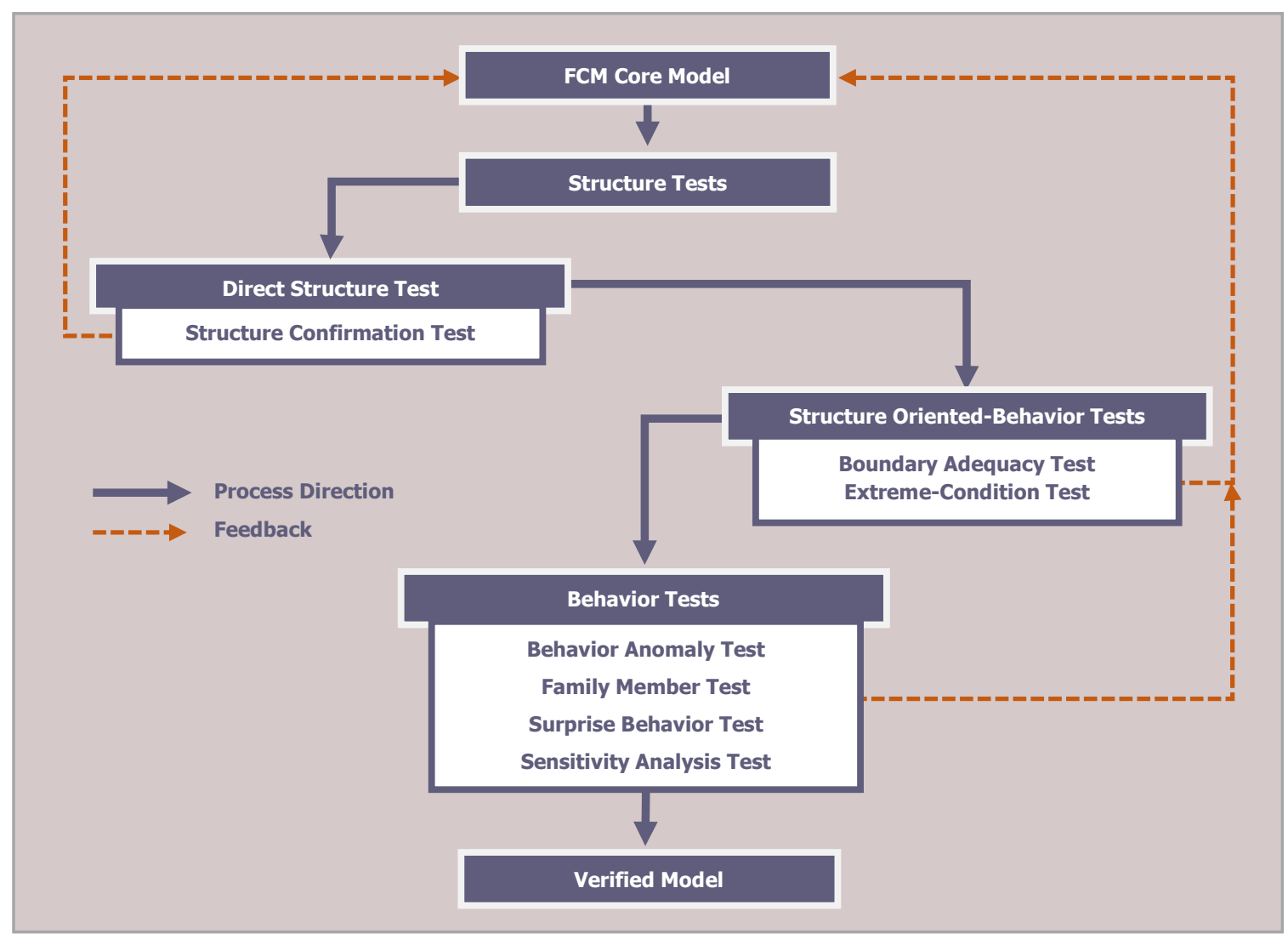

Figure 15: The Process of Model Verification 


\subsubsection{Structure Tests}

Testing the model structure refers to examining whether the model's relationships are an appropriate representation of the real relationships concerning the study's purpose (Barlas, 1996; Sterman, 2000). In this research, the model's concepts and weighted causal relationships that link them and aggregate the model structure are fully documented using various computer aid software tools, namely "Atlas.ti" and research memos. Although the documentation process is essential to ensure the quality of the model structure (Saunders et al., 2015), verifying the model structure requires not only documentation but several techniques using formal verification tests, by which the concepts of the model and the causal relationships among them tested using direct structure tests and structure-oriented behavior tests, which are discussed as follows.

\subsubsection{Direct Structure Tests}

Direct structure tests can be carried out by directly comparing the model's structure with the available knowledge (Barlas, 1996). There are few tests under this category of model structure testing, including the following structure confirmation test applicable to test FCM models' structure.

Structure Confirmation Test: This test inspects the accuracy of the model structure. It focuses on the aggregation model level to examine the structure for discrepancies and faulty assumptions regarding how the model was built. This test is conducted using submodel diagrams (Sterman, 2000), by which a direct inspection is carried out to each of the thematic networks (sub-model) individually and then collectively. This test assesses the aggregation's assumptions by conducting (1) a theoretical comparison with the generalized 
knowledge in the literature and (2) an empirical comparison with the available information regarding the applications of the HROT in various industrial settings.

\subsubsection{Structure-Oriented Behavior Tests}

These tests involve simulations, and they assess the accuracy of the model structure indirectly by applying behavior tests to uncover potential issues in the structure. Various tests were used to examine the structure-oriented behaviors, but according to each test's explanation, few were applied to the FCM modeling technique. In this regard, I am using the boundary adequacy test and extreme condition test, which are discussed as follows.

Boundary Adequacy Test: This test evaluates the appropriateness of the model boundaries for the purpose it was built for. Conducting this test reveals whether the model boundaries should be expanded or disaggregated to achieve the model purposes by comparing the model boundaries with the scope of the phenomena under study. A model boundary chart is prepared to distinguish between the internal elements (endogenous-variable) and external elements (exogenous-constants). The model then simulated to examine whether essential feedback(s) in the model have been omitted. In other words, to determine whether exogenous concepts have been treated as endogenous and vice versa.

Extreme-Condition Test: This test examines the model's responsiveness and robustness to extreme scenarios and shocks. This stress testing involves the assignment of extreme values to specific parameters and compares the output behavior of the model with the observed or predicted behavior of the phenomena under the same settings. This test runs a simulation based on various scenarios and inspects whether the outputs of such scenarios are feasible and reasonable even when the input is at a maximum level or a minimum level. Carrying 
out this test can be through designing various strategies. The model is subjected to a high level of stress; therefore, issues in the model structure reveal its failure to mimic the realsystem structure.

\subsubsection{Behavior Tests}

Testing the model behavior examines whether the model can demonstrate close enough behaviors to the observed real behavior (Barlas, 1996; Sterman, 2000). While examining the model behavior can be meaningfully conducted after examining and verifying the model structure, the model-generated behaviors resulting from testing the structure can establish an initial indication of the behavior's verification. The model behaviors would be tested using different techniques to assess how precisely the model replicates a phenomena's behaviors. The following tests were used to examine the model behaviors.

\subsubsection{Behavior Anomaly Test}

This test examines the model behaviors for any abnormal patterns that may arise when the model's assumptions are changed or deleted. Showing an anomalous behavior when a relationship or a specific causal path is deleted or modified reveals whether this relationship/path is essential or not. This test is conducted using a knockout loop analysis (Sterman, 2000), by which zero out critical effects in the model. The test requires activating a state vector with varying activation levels for a driver concept that directly impacts the examined relationship while setting the relationship between the chosen concepts to zero, or in other words, no connection. The resulted model behaviors show the level of importance of this relationship in the model structure for the purpose it was built for. 
Arbitrary selecting two to three paths to examine based on this test procedure can lead to a general understanding regarding the importance of the model causal links.

\subsubsection{Family Member Test}

This test examines the model's robustness and capabilities to generate behaviors for other cases in the same class that are similar to the system that the model is built to represent. The diversity of the cases that the model can represent reflects the generalization of the theory it embodies (Sterman, 2000). Since the model is built to represent the HROT theory, the model's behaviors should serve different organizational settings with other policies, strategies, and even various industries. This test is conducted using random data that represent different organizational or industrial operational settings. If the model generates output that matches each input's expectations, the model, therefore, passes the test.

\subsubsection{Surprise Behavior Test}

This test uncovers discrepancies between the model behaviors and the modeler's understanding of a phenomenon. When the model generates a specific behavior that has not been observed but can exist in the real world, the model can pass the test.

\subsubsection{Sensitivity Analysis Test}

This test inspects the model sensitivity to the likely changes and variations in the model's parameters' values. Suppose the model does not show radical or severe behavioral changes, the confidence around the model increases. There are three types of sensitivity: (1) numerical sensitivity, which results when a difference in the assumptions changes the numerical value of the parameters, (2) behavior mode sensitivity, which results when a change in the assumptions changes the behavioral patterns of the model, and (3) policy 
sensitivity, which results when a difference in the assumption reverses the impact or the desirability of a proposed policy. Because the model faces additional challenges represented by the self-quantifying-edges, it is essential to carry out a sensitivity through the following two sequential steps.

1. Test the model without changing the assumptions by activating state vectors with varying activation levels for a single concept at a time and then randomly changing a bundle of concepts in the input vector to examine the range of the possible outcomes for all the five HROT principles.

2. Repeat the same process while proposing some changes in the model assumptions and compare the results with the first step.

\subsection{Model Simulation and Scenario Planning}

Model simulation is a process of imitating the modeled system by activating one or more of the model concepts to analyze and understand the immediate change or the dynamics in the system components and or predict its future state. Model simulation can be conducted by planning various scenarios to study the system responses, such as changing the policies or rules and regulations. The following discussions define and explain both model simulations and scenario planning.

\subsubsection{Model Simulation}

With respect to the developed research framework (Figure 1), organizational reliability (OR) or collective mindfulness is a function of change in both mindful anticipation and mindful containment or the change in the five HROT principles. However, organizational 
reliability cannot be achieved by simply maximizing all concepts that lead to desired effects and minimizing all those that do not. It is often unknown which concepts lead to what effect, especially that many concepts cannot be changed directly but only through other concepts. Because concepts are interrelated, changing the desired ones often also impacts undesired ones. It is, therefore, difficult to determine a course of action. This can only be achieved by simulating the model based on various combinations (scenarios) of desired and undesired concepts (i.e., HRO-relevant concepts).

Importantly, the model of interests (i.e., the complete model of HROT) consists of (1) a core model, which describes the concepts that contribute to the five HROT principles (based on the literature) and (2) an outer layer that represents how specific safety interventions impact the concepts on the boundary of the core model. The concepts on the core model's boundary do not change through anything in the core model but, instead, trigger changes in the core model if they are externally increased or decreased. This can be possible by simulating the model to investigate the dynamics of the HROT principles, regardless of interventions (by simulating the core model) and the impact of interventions on HROT principles (by simulating the complete model of HROT). The following two methods of simulation are used in this research.

1. Method A: Simulating the core model regardless of intervention(s), through which traditional FCM simulation technique is used.

2. Method B: Simulating the model of interests based on intervention(s), through which both traditional FCM and EMA simulation techniques are used. 
Method $\boldsymbol{A}$ is used in the process of model testing for verification, while Method $\boldsymbol{B}$ is used in the process of model contextualization. Each method is implemented using three different approaches: A, B, and C, as explained in detail in Table 7 below. Furthermore, before simulating the model, a squashing function must be selected that best fits the model's expected behaviors, modeling objectives, and the modeler's plans to present and visualize the outcomes (Alibage et al., 2018). The selection of the squashing function and Lambda value is decided based on an experiment or a pilot study.

Moreover, various software-based tools can be used to simulate the model. In addition to Microsoft Excel by Jetter et al. (2018), there is an FCM Package-based Python programming language by Aminpour (2018), as well as FCM-package based R programming language by Dikopoulou \& Papageorgiou (2017). 
Table 7: Combinations of Model Simulation Methods and Approaches

\begin{tabular}{|c|c|c|}
\hline N & $\begin{array}{c}\text { Method A } \\
\text { (Simulating the Core Model Without Intervention) } \\
\text { Traditional FCM Simulation }\end{array}$ & $\begin{array}{c}\text { Method B } \\
\text { (Simulating the Model of Interest Based on Intervention) } \\
\text { Traditional FCM Simulation } \mid \text { EMA Simulation }\end{array}$ \\
\hline Approach $A$ & $\begin{array}{l}\text { This approach is used to reflect a particular idea about starting } \\
\text { conditions. It involves selecting a state vector with one or more } \\
\text { activated core model's concepts while the other concepts remain } \\
\text { off. The activation level is anything of choice, and the selection is } \\
\text { guided by a question that can be answered through the simulation. }\end{array}$ & $\begin{array}{l}\text { This approach uses traditional FCM simulation and involves } \\
\text { selecting a state vector to activate one or more interventions that are } \\
\text { causally linked to the core model's concepts. The activation level is } \\
\text { anything of choice (between } 0 \text { and 1) to investigate the impacts of } \\
\text { specific interventions on HROT five principles' performance. }\end{array}$ \\
\hline Approach B & $\begin{array}{l}\text { This approach involves selecting a state vector with one or more } \\
\text { activated core model's concepts while the other concepts remain } \\
\text { off. Because the interest is to analyze the results of different input } \\
\text { vectors and their impacts on simulation results; thus, the process } \\
\text { is repeated for the same vector but with varying activation } \\
\text { degrees. This approach examines the sensitivity of the concepts of } \\
\text { interests to the variations in the inputs. }\end{array}$ & $\begin{array}{l}\text { This approach uses traditional FCM simulation. It involves selecting } \\
\text { a state vector to activate one or more interventions that are causally } \\
\text { linked to the core model's concepts. The process is repeated for the } \\
\text { same vector but with varying activation degrees to investigate the } \\
\text { results of different input vectors and their impact on the aggregated } \\
\text { simulation results to examine the sensitivity of the concepts of } \\
\text { interest variations to the inputs' variations. }\end{array}$ \\
\hline \multirow[t]{2}{*}{ Approach C } & $\begin{array}{l}\text { This approach involves selecting different state vectors and } \\
\text { randomly activating a specific number of the model's concepts } \\
\text { (e.g., five concepts), excluding the concepts of interest. The aim } \\
\text { of using this approach is to analyze the overall pattern of the } \\
\text { modeled system's behaviors. }\end{array}$ & $\begin{array}{l}\text { This approach employs the EMA simulation technique and is used } \\
\text { when there are variations in the signs and the strengths of one or } \\
\text { more causal relationships between the interventions and the core } \\
\text { model's concepts. This technique simulates a vast number of the } \\
\text { core model's structures instead of only one so that it copes with the } \\
\text { variations (uncertainties) in the inputs. }\end{array}$ \\
\hline & Used in the Process of Model Testing for Verification & Used in the Process of Model Contextualization \\
\hline
\end{tabular}




\subsubsection{Traditional FCM Simulation}

FCM models can be simulated traditionally by generating initial state vectors based on the number of the concepts in the model $(\mathrm{N})$ and their initial values, which often can be [0] for deactivated concepts and [1] or [-1] for activated concepts. Therefore, initial state vectors for an FCM with $(\mathrm{N}=50)$ based on the formula $\left(2^{\mathrm{N}}\right)$ can produce $(2.2517998 \mathrm{e}+15)$. However, it is neither wise nor practically possible to simulate the model based on this vast number of initial state vectors, particularly with the meta-rule of FCM, which means that many different inputs can lead to the same outcome. Instead, initial state vectors must be chosen deliberately to produce a state of interest. The chosen initial state vectors can either directly activate one or more of the model concepts or one or more interventions and then run the FCM for initial values. This technique is used when the model is simulated during the process of model testing for verification and the process of model contextualization (see Table 7).

\subsubsection{EMA Simulation}

FCM models can be simulated using the EMA technique to overcome potential uncertainties in the model structure, particularly the variations in the strength of the causal relationships between the interventions and the model's driver concepts. This technique is conducted using the following steps.

1. Test the sensitivity of the concepts of interest to the variations in the edges' weights between the interventions and the driver concepts in the core model' boundary.

2. In cases where the concepts of interest are sensitive to the variations in weights of the examined edges, the model is then simulated using EMA, through which random 
adjacency matrices are generated to include all the possible values between the minimum and maximum edge weights.

This technique is used when the model is simulated during the process of model contextualization (see Table 7).

\subsubsection{Visualizing the Outcomes}

There can be various types of graph representations to visualize the outcomes of the concepts of interest, particularly with the use of programming FCM-packages. As mentioned previously, visualizing the simulation results depends on the choice of the squashing function and Lambda's value. In the interest of this research, three types of graphs will be used: Bar graph, sensitivity analysis graphs, and radar charts.

\subsubsection{Scenario Planning}

A scenario is defined as "a set of hypothetical events set in the future [and are] constructed to clarify a possible chain of causal events as well as their decision points" (Kahn \& Wiener, 1967, p. 6). It is a dynamic explanation that describes the key fundamentals of uncertainties or unforeseen futures of a system under study (Nyaupane \& Buzinde, 2017). Planning scenarios is a widely used technique whereby decision-makers can elaborate on their mental models regarding making better decisions based on plausible futures rather than the possible ones (Amer et al., 2013). Scenario planning can be applied in the context of systems safety as a valuable technique, particularly in predicting unexpected events or the combinations of complex issues or future situations (e.g., implementing fully automated operations, reduction in experts availability) that test the system readiness, resiliency and responsiveness to such cases and potential consequences. In high-hazard 
organizations, scenario planning provides plans for worst-case scenarios so that organizations can develop subsequent preventative actions to mitigate harmful impacts. Amer et al. (2013) discuss and compare various approaches and methodologies to scenario planning. However, they emphasize two major approaches: A qualitative approach that encompasses the use of subject experts to develop and explain scenarios, and a quantitative approach that involves modeling. However, they recommend combining both approaches as a better means to create robust scenarios (Amer et al., 2013). Since this research uses fuzzy cognitive mapping FCM, therefore, I am using a quantitative approach for scenario planning, through which the following steps are required to develop, select and refine scenarios based FCM (Amer et al., 2013): Scenario preparation, Knowledge capture (identify key concepts and drivers), Scenario modeling (develop a causal map with weighted causal links), Scenario development (using a different set of inputs vectors to see the behavior of the FCM model), Scenario selection and refinement, and Strategic decisions.

The number of alternative scenarios varies between two to six, yet, a wide range of scenarios can be developed to accomplish the overall objective of this research, by which organizational reliability is optimized in the face of unexpected events before, during, and after they occur. Moreover, simulating the FCM does not provide absolute values of the performance, but rather, it provides degrees of change in the performance. Because there is no available information regarding which degree the organizational reliability is considered high, a high degree of organizational reliability can be determined by comparing the simulation outcomes of different scenarios. 


\section{The Process of Building the Core FCM Model}

The previous chapter provided an overview of the methodological foundations of this work. This chapter discusses how these methods were combined to answer the first three research questions (see Table 4) and develop a mixed-method approach for modeling HROT principles as FCM, based on published literature. This approach was subsequently implemented for the specific case of HROs in the offshore oil and gas industry during a pilot and an expanded study, which are reported on in subsequent chapters. As shown in Figure 16, my process for building the FCM model consisted of two research phases with multiple steps each. In step 1 of the first phase, I performed a systematic literature review to search for and select published research on HROT based on predefined criteria and review guidelines. In step 2, I extracted and refined the qualitative knowledge by conducting a structured qualitative coding process, followed by step 3, through which I performed a structured theming process, which resulted in creating the research collective cognitive map (CM).

In the second phase, the research transitioned from qualitative to quantitative work: I quantified the causal links of the cognitive map developed in the first phase by employing another structured process that consisted of three steps. Step 1 included retrieving and filtering shared content, step 2 included calculating edges' weight, and step 3 included examining the relevance of the used technique. This allowed me to convert the cognitive map into a fuzzy cognitive map (FCM) entirely based on the literature. The following sections present the process of model building and its implementation. 


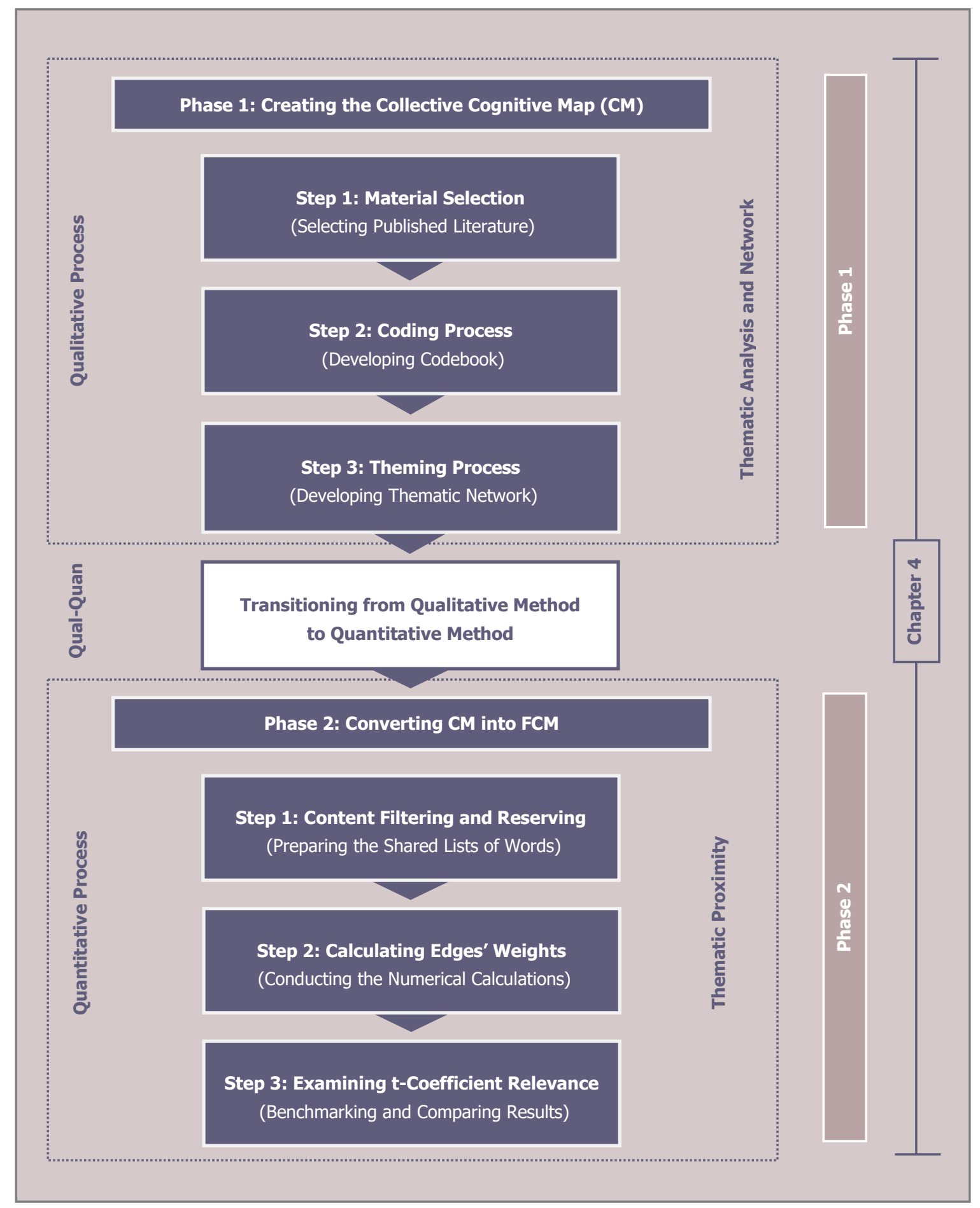

Figure 16: The Process of Building the Core FCM Model Based HROT Principles 


\subsection{Phase 1: Creating the Collective Cognitive Map (CM)}

In phase one of this research, I created a cognitive map representing the concepts and causal links between concepts discussed in the HROT literature. It provides the starting point for the creation of the FCM model in phase 2. To ensure that the cognitive map fully and accurately represents the state of the literature, I used a systematic, transparent, and fully documented process, based on the principles of thematic analysis (Braun \& Clarke, 2006; 2014), and thematic network (Attride-Stirling, 2001), discussed previously. To support this work, I used Atlas.ti, software for qualitative data analysis.

The work took place in three steps. The first step involved defining criteria and selecting research publications on HROT to be included in the analysis. In the second step, the

selected publications were carefully reviewed and coded. Simultaneously, a codebook of the most significant concepts and relationships between them was developed to document and clarify their meaning. In the third step, the codes are "themed" (i.e., combined into broader categories or themes), based on their meanings and relationships, resulting in a thematic network (or cognitive map) of causally related concepts. This network represents the overall cognitive map. The following sub-sections discuss these steps in more detail.

\subsubsection{Step 1: Selection of Published Research}

I used a systematic literature review to select a list of publications that represents the HROT's state-of-the-art. Unlike traditional or narrative literature review, which seeks to critically examine and critique the status and gaps of published literature on a specific topic, a systematic literature review is defined as: 
"a review of a clearly formulated question that uses systematic and explicit methods to identify, select, and critically appraise relevant research, and to collect and analyze data from the studies that are included in the review" (Moher et al., 2009, p. 1006). The objective is to select published research that meets pre-defined criteria to ensure quality and content validity and to cover not only the theoretical principles of HROT but also empirical research and theory applications in various industrial settings.

In this step, I used Preferred Reporting Items for Systematic Reviews and Meta-Analyses PRISMA guidelines by Moher et al. (2009). These guidelines provide a basis for an improved systematic review by which I followed a checklist of multiple items and a fourphase flow diagram. However, the checklist used is not a tool that assesses the systematic review quality (Moher et al., 2009). Since the scope of this review is to select online publications rather than conducting statistical analysis, this review does not cover the part of the so-called meta-analysis. The following discussions explain how I accomplished this step.

\subsubsection{Searching Strategy}

I followed a strategy for searching publications using five online databases: Scopus, ScienceDirect, Academic Search Premier, Web of Science, and Google Scholar during 1987 when the first project on HROT was published until 2018 when this search was conducted. I used various keywords, including "high reliability organizations," "high reliability theory," "HROT," "HROs," "organizational reliability," "organizational mindfulness," and "mindful organizing" to search for documents in the English language 
only. The initial search yielded (562) research items included various document types with multiple contents, objective(s), methods, and findings.

\subsubsection{Selection Strategy}

Following the PRISMA guidelines discussed above, I subjected the initial search outcome of (562) documents to a screening process based on the predefined inclusion/exclusion criteria listed in Table 8.

Table 8: Predefined Criteria for Selecting Publications on HROT

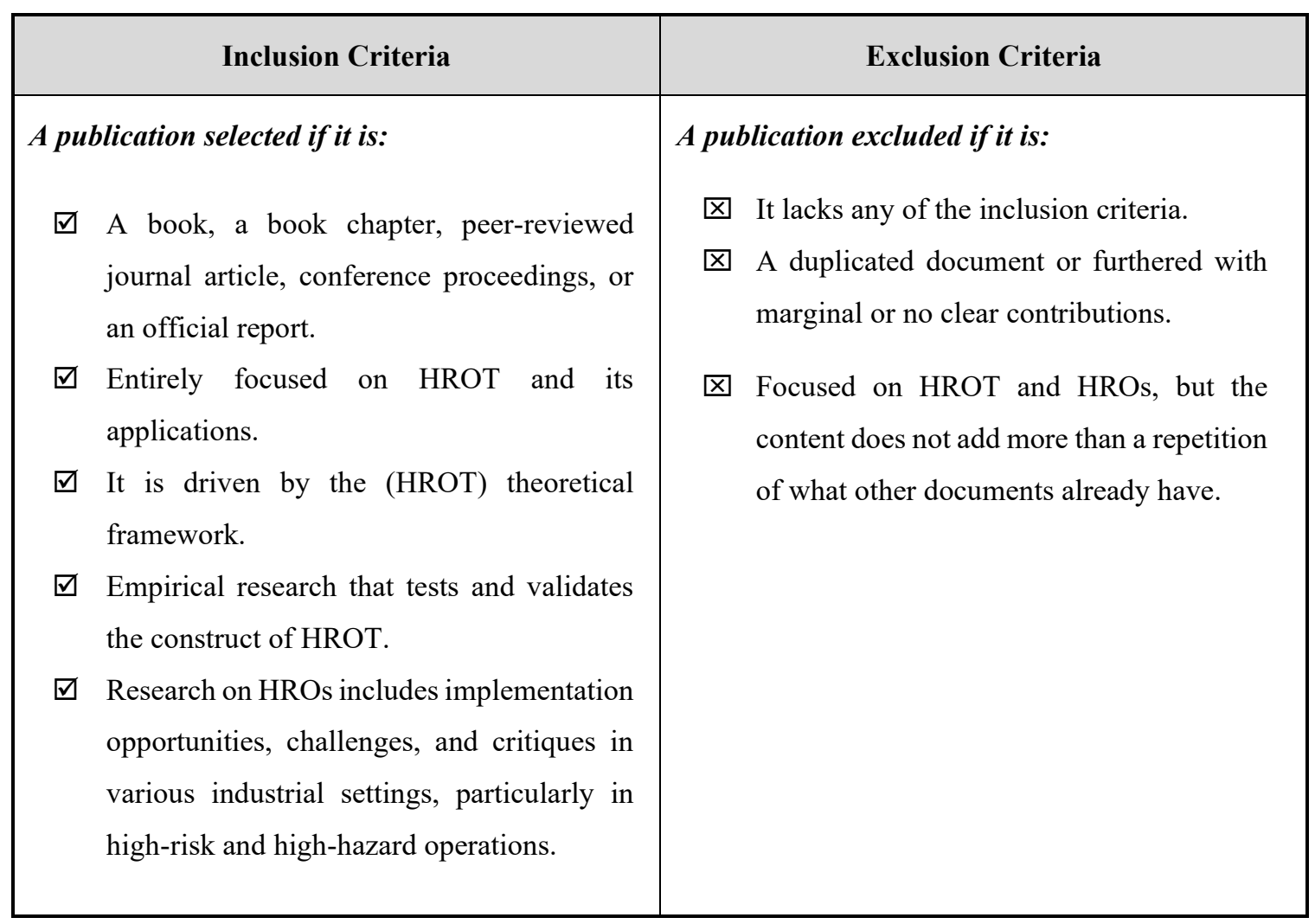

By refining the search into peer-reviewed journal papers, conference proceedings, books, books chapters, and official reports, the search outcome was reduced to (139) documents including (63) peer-reviewed journal articles, (54) conference proceedings, (7) book series, (6) books, and (9) official reports. However, publications that met the inclusion criteria and 
screening process were only (47). Table 23 in Appendix B: Selected Research Publications includes the list of the selected studies to conduct the qualitative phase of this research, and Figure 17 below graphically illustrates this process.

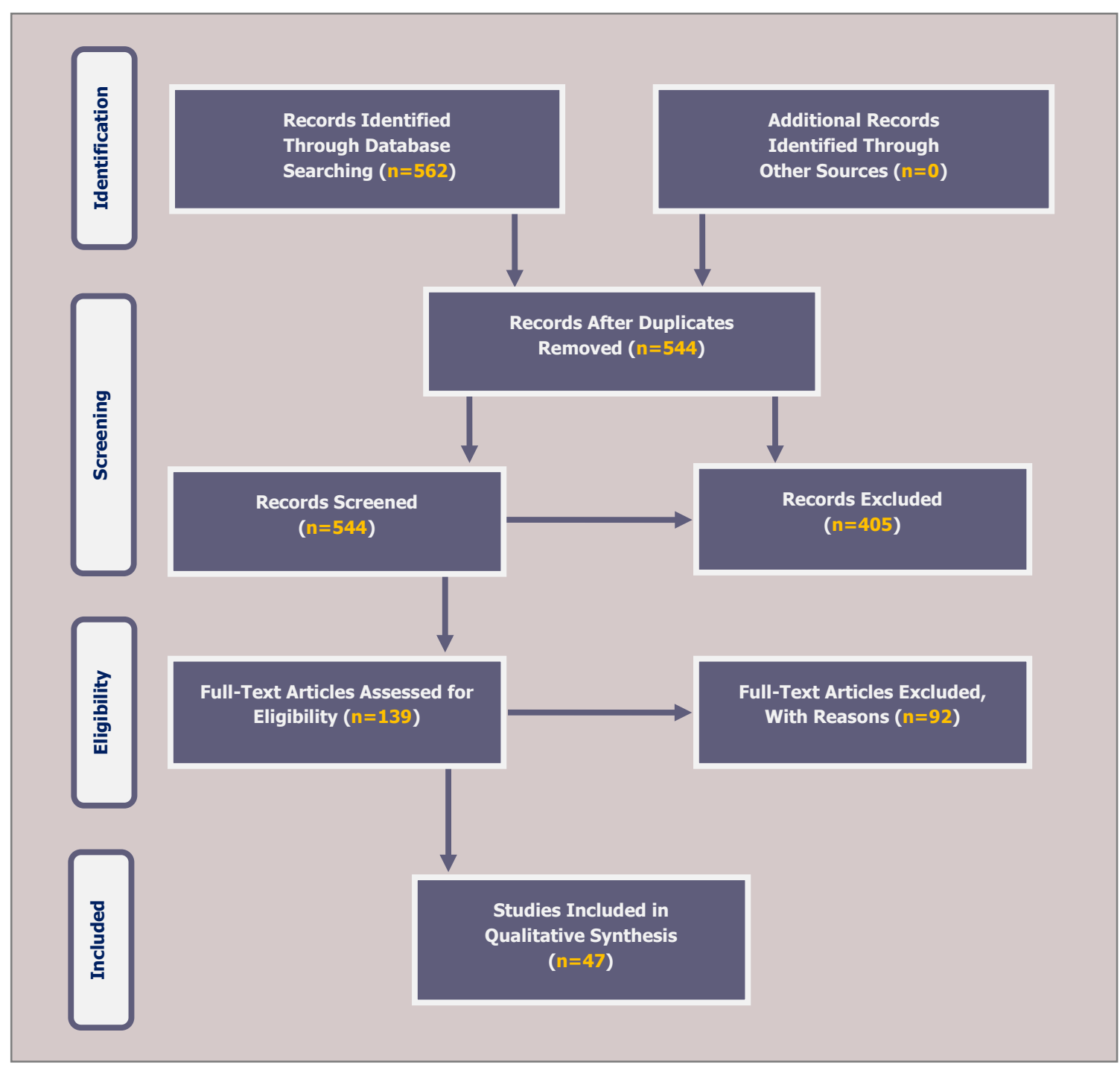

Figure 17: The Process of Selecting Relevant Publications 


\subsubsection{Step 2: Coding Process}

Coding in qualitative analysis is a process of segmenting the data ${ }^{13}$ into various segments and labeling them based on the meanings they embody to understand the relationships among them better. This process goes beyond just segmenting the data into a broader and in-depth analysis. The extracted knowledge is refined iteratively to create ideas that make sense when interpreted based on a structured pathway. Text segments vary in terms of their length as they can be one sentence or a paragraph, and they usually are labeled with a single word or a short phrase (i.e., code) that is either chosen from the segment itself or that is given by the coder. Some segments can have more than one label (code), particularly when conveying various and overlapped meanings.

Since the objective is to create a causally-descriptive cognitive map that represents the complex and dynamic phenomenon of organizational reliability, the process of coding is designed to identify the most significant factors across the whole set of the selected text that contributes in a causal construct to the creation of the five HROT principles. The process also identifies factors that preclude achieving organizational reliability. This step involves the selection of a coding approach and coding strategy.

\subsubsection{Coding Approach}

There are various coding approaches to be used when conducting qualitative research. Yet, the suitability of each approach depends on the research method since the process of coding

\footnotetext{
${ }^{13}$ Qualitive data can be interview transcripts, participant observation, field notes, journals, documents, open ended survey responses, drawings, artifacts, photographs, videos, internet sites, email correspondence, academic and fictional literature, and so on (Saldana, 2016)
} 
is the foundation for any qualitative inquiry. Because this research uses thematic analysis (TA), I selected a codebook approach ${ }^{14}$ for its relevance to this research's scope and objective. This approach ensures reliability and allows flexibility as both approaches are essential for analyzing unstructured text and adopt a dual coding strategy.

\subsubsection{Coding Strategy}

Based on the coding manual for qualitative researchers by Saldaña (2016), I followed two strategies to code the data: Deductive and inductive coding. Deductive coding means applying a predefined list of codes (concepts) that have been identified based on reviewing and understanding the whole set of text. This strategy tests and verifies the significance of each predefined analytical unit in terms of its grounding in the selected literature and relevancy to the general definition of the HROT five principles. However, inductive coding searches for possible and additional significant and relevant concepts that emerge from the data during the implementation of the coding process, including meanings and descriptions of what makes and precludes the phenomenon's development.

Following either strategy or both of them necessitates the following coding heuristics ${ }^{15}$, which I browed from Armborst (2017) as guidelines to conduct the coding process:

\footnotetext{
${ }^{14}$ Three coding approaches suggested to conduct thematic analysis TA (Clarke, 2017): Reliability, reflexive, and codebook. Reliability approach is used when the coding process is highly structured and guided by a coding frame and when the emphasis is on ensuring accuracy. The coding structure of this approach is usually developed prior to the analysis and then applied to the data. The reflexive approach consists of a high level of flexibility since the emphasis is on engagement with the data and the depth of the interpretation. Therefore, coding is free of any structured frames, which means that codes continuously evolve, change, rename, split, and merge until they make sense with respect to the objective of the interpretation. However, the codebook approach is in the middle of the two discussed approaches.

${ }^{15}$ Coding heuristics are defined as "standardized rules that guide the decision of the researcher about when to create a new analytical unit, how to label it, and how to separate codes, categories, and themes from each other" (Armborst, 2017, p. 2).
} 
1. Internal Homogeneity: to maximize cohesive validity, by which all the contents of one unit (code and later theme) are similar.

2. External Heterogeneity: to maximize the discriminant validity, through which the contents of two different units (code and later theme) are about two different things.

3. Code Overlaps: to follow heuristics 1 and 2; codes overlap as low as possible and as often as needed.

\subsubsection{Coding Procedure and Outcomes}

Following the codebook approach and the coding heuristics discussed above, I conducted the process of coding using Atlas.ti software and employing both inductive and deductive strategies across two cycles of coding (Saldaña, 2016). Figure 18 illustrates how the overall coding process was conducted. The following sections discuss the coding process in completing the first and second cycles of coding and its outcomes.

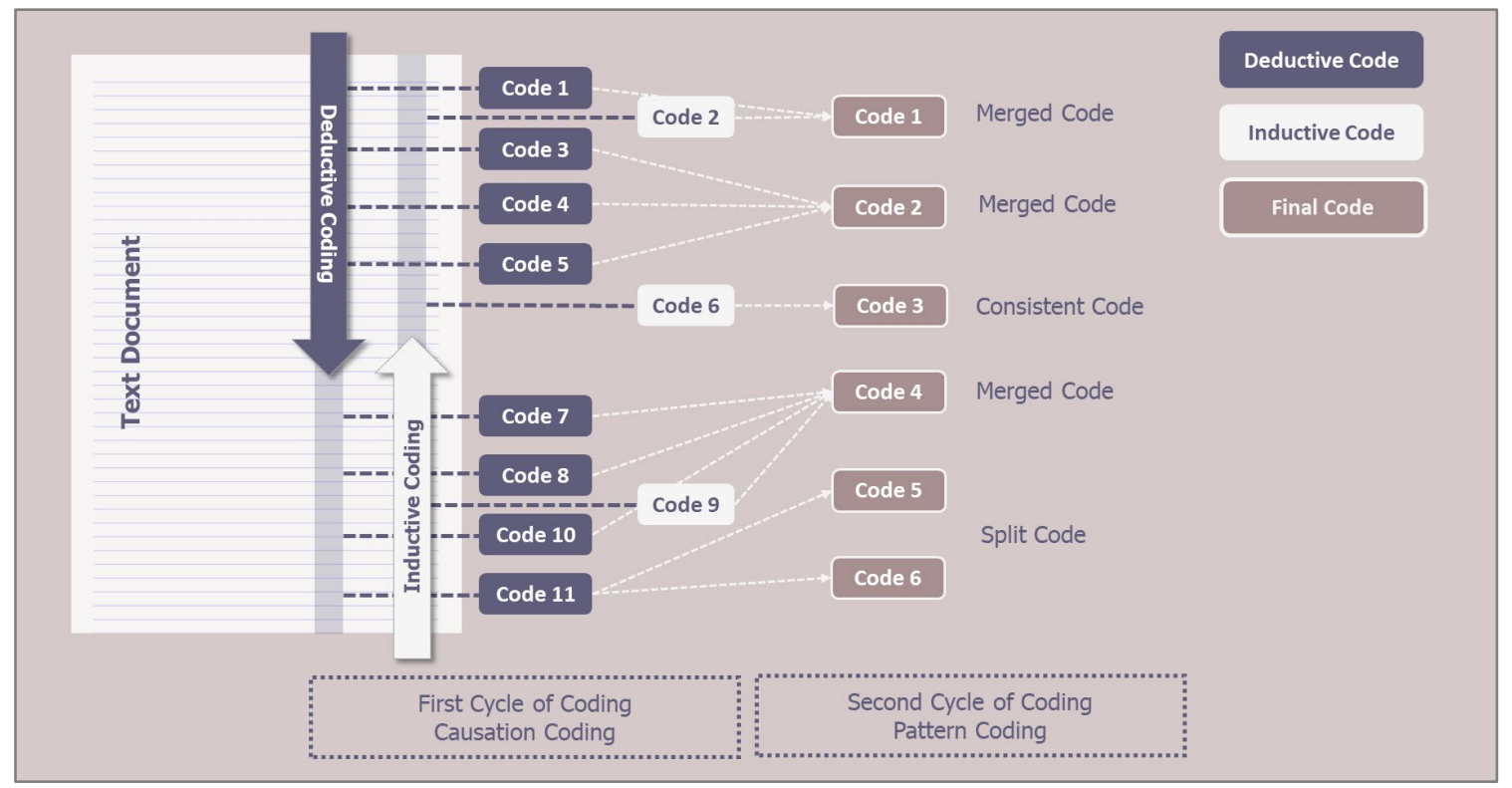

Figure 18: The Process of Coding Textual Data 
First Coding Cycle: Since the objective is to create networks of causally related components; therefore, "causation coding" was used in this coding cycle. This type of coding "recommends procedures for extracting attributions or causal beliefs from participants data about not just how but why particular outcomes came about" (Saldaña, 2016, p. 174). Causation coding can be used to examine causalities among variables, for mediating variables, and for both within and between literature analysis. Therefore, it can be shaped into a causation model. It is classified under the procedural coding method, which comprises a pre-established coding scheme or ways of analyzing qualitative data. This method explains why a situation happens by identifying combinations of specific conditions and the variables that mediate between them.

Figure 19 is a screenshot of Atlas.ti that explains how documents were coded, and causalities were identified between pairs of codes. In this example, few causalities can be elicited from this small portion of text. From this example, it is evident that success breeds "Complacency," which is a state of overconfidence that increases the possibility of undiscovered events to go through the system and accumulate towards accidents. I labeled such events' potentials and all similar concepts as "Cues of Evolving Failure," while I labeled complacency and similar concepts with the same label when coded. Both "complacency" and "cues of evolving failure" were uniquely defined, and their relationship is documented in a separate and numbered research memo ${ }^{16}$ for quality control purposes.

\footnotetext{
${ }^{16}$ A research memo is a note-based review or observation that can be placed anywhere in the project to document an important issue, which in the case of this research was attached to every pair of causally related codes as a technique to ensure quality and validity of the coding structure (Saunders et al., 2015).
} 
The first cycle of coding resulted in a list of 134 uniquely defined and causally related codes with a total of 435 connection (positive and negative) based on five code families that represent the five HROT principles.

D Code Manager Di. D 2: P2-(Weick \& Sutcliffe, 2015) $~ \times$

Success narrows perceptions, changes attitudes, reinforces a single way of doing things, breeds overconfidence in current practices, and reduces acceptance of opposing points of view. The problem is that if people assume that success demonstrates competence, they are more likely to drift into complacency and inattention. What they don't realize is that complacency also increases the likelihood that unexpected events will go undetected longer and accumulate into bigger problems.

\section{1}

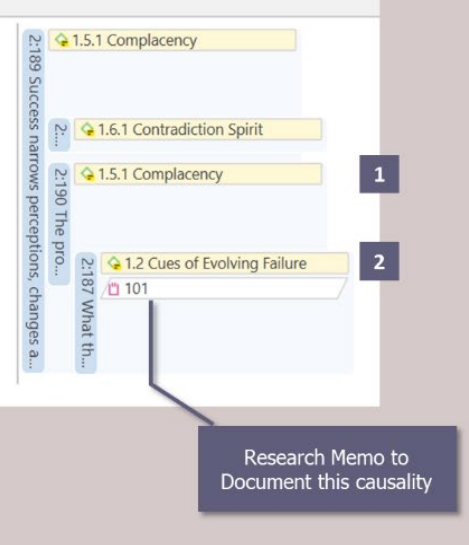

Figure 19: Screenshot of Atlas.ti to Show How Documents Were Coded

Second Coding Cycle: In this coding cycle, I used pattern coding to search for commonalities and similarities among the generated codes during the first coding cycle. This cycle synthesized the developed list of codes by (1) merging codes that implied similar or common meanings and descriptions and had corresponding impacts within their context, and (2) by splitting codes that were comprised of complex meanings and interpretations into two or more codes within their context. The objective was to ensure that all codes (deductive and inductive) were unique, distinctive, and exclusively defined, by which potential duplications and undesired repetitions were eliminated. The final codebook that resulted from this cycle consisted of 112 causally related and uniquely defined codes with 409 positive and negative connection based on five codes families 


\subsubsection{Coding Reliability}

The process reliability ${ }^{17}$ of coding qualitative data refers to the consistency in conducting the coding process by a single or multiple coders overtime. It can be measured by duplicating the research efforts under different conditions, such as different times, and then examining the similarities and differences in readings, interpreting, and analyzing the data under study (Krippendorff, 2004).

According to Braun et al. (2016), agreement and the use of Inter-Rater Reliability (IRR) ${ }^{18}$ within qualitative methods that heavily rely on interpretation, such as thematic analysis, is not advocated. The coding process in TA is flexible, and codes evolve throughout the process to reflect the coders' engagement with the data; therefore, agreement of two or more coders around specific codes does not mean that they are valid (Braun et al., 2016). Furthermore, McDonald et al. (2019) conclude, based on reviewing the literature on qualitative methodologies, that researchers may not seek agreement and IRR when coding is the process rather than the final product as in TA.

The TA's primary goal is not to categorize data but to inductively identify concepts and themes and uncover meanings representing a phenomenon. However, in my work, I used TA inductively to identify thematic networks and determine the weight of the causal relationship between each two causally related themes (see Section 3.5), which constitutes

${ }^{17}$ According to Krippendorff (2004), there are three types of coding reliability:

- Replicability of results across coders (i.e., Inter-Rater Reliability (IRR)), is a statistical measurement to establish an agreement between two or more coders.

- Stability or consistency of a single coder overtime (i.e., Intra-Rater Reliability).

- Accuracy of an established coding scheme compared with others.

${ }^{18}$ Agreement and Inter-Rater Reliability (IRR) stems from realist/positivist assumption and infers that there is a precise reality in the data that possibly captured through the process of coding (Mackey \& Gass, 2005; Cook, 2011). 
an innovative approach to creating FCM models from texts. Therefore, I decided to use Intra-Rater Reliability to ensure stability or consistency in the process of coding. IntraRater Reliability ensures that data is judged the same way when the same researcher codes the same data at different times (e.g., time $\mathrm{X}$ and time $\mathrm{Y}$ ) or even at the beginning and the end of the process (Philp, 2003; Mackey \& Gass, 2005).

I employed a Test-Retest technique and subjected the same selected material to the same coding process, but as a minimum two weeks apart. I then calculated the coding reliability using a Simple Percentage Score that compares the degree of agreement resulting from the two coding attempts. An interval of two weeks is assumed to be sufficient to reveal any disagreements between the two attempts' outcomes (Mackey \& Gass, 2005). Therefore, the coding process was repeated after two weeks, which resulted in a disagreement around (9) codes, while (103) final codes were approved. Based on that, IRR was calculated as (91.96\%). According to Mackey \& Gass (2005) and Riffe et al. (2014), anything above $75 \%$ is considered acceptable, although percentages over $90 \%$ are ideal. Table 9 consists of the codes and connections numbers across all the steps of the coding process.

Table 9: Number of Codes and Connections Over the Steps of the Coding Process

\begin{tabular}{|l|c|c|c|}
\hline $\begin{array}{l}\text { Coding Stage } \\
\text { Connections }\end{array}$ & $\begin{array}{c}\text { First Coding Cycle } \\
\text { (Causation Coding) }\end{array}$ & $\begin{array}{c}\text { Second Coding Cycle } \\
\text { (Pattern Coding) }\end{array}$ & $\begin{array}{c}\text { Post Coding Reliability } \\
\text { (Intra-Rater Reliability) }\end{array}$ \\
\hline No. of Codes & 134 & 112 & 103 \\
\hline No. of Causal Links & 435 & 409 & 368 \\
\hline
\end{tabular}


The list of the generated and approved final codes in the developed codebook, which consists of 103 uniquely labeled and defined codes based on five code families, is provided in Table 24 in Appendix C.

\subsubsection{Step 3: Theming Process}

Qualitative theming is a process of thoroughly examining the data under study to search for patterns (themes) of meanings that are related to the research question(s) at hand. This process simplifies, organizes, and interprets the complex content of the data into more understandable issues based on their interrelationships' structure. Therefore, a theme is an abstract description of a set of interrelated issues (Armborst, 2017), and technically, as illustrated in Figure 9, it is a product of qualitative coding (Saldana, 2016).

Themes are generated by grouping codes based on their causal relationships and causing an outcome. For example, acoustic instruments such as guitars and violins (assumed as codes) are distinct by design, material, use, and even musical tones. Yet, they produce acoustic music (i.e., the theme of music). The following sections discuss the strategy and procedure that I followed in conducting the process of theming in more detail.

\subsubsection{Theming Strategy}

Building on the objective of the coding process, the aim of theming the selected published research is to identify the most significant and causally related factors that enable implementing and continuously sustaining the five HROT principles and the factors that prevent a successful and consistent implementation. Therefore, I employed a theming strategy that uses domain summaries or storytelling themes (Clarke, 2017). Both desired 
and undesired factors and their relationships and joint effects around the central concept (i.e., organizational reliability) are considered.

Since the five interrelated principles characterize HROT as a structured pathway for conducting the coding process, this structure continues to be the foundation for the theming process. The five principles are treated as global themes. Each theme consists of or is caused by a varying number of causally related organizing and basic themes as lower and middle order themes that are generated based on the developed codebook.

\subsubsection{Theming Procedure and Outcomes}

I conducted the theming procedure following the same heuristics discussed in Section 4.1.2.2 as guidelines that ensure internal homogeneity, external heterogeneity, and theme overlap. As previously mentioned, I considered each of the five HROT principles as a global theme, and I followed the thematic arrangement discussed in Section 3.4. Each principle, thus, formed a web-like network (Attride-Stirling, 2001). The following substeps discuss the overall procedure of theming.

First: Examining the Final Codes: I examined the final codes within each coding family (principle) regarding their definitions and causalities as well as their potential in causing a particular outcome. Codes with potential theming attributes were themed as basic themes (lower-orders) or organizing theme (middle-order) and were labeled and defined accordingly. However, final codes that are unique and do not share similar or general concepts or the same outcomes within their families were converted into either a basic or an organizing theme, depending on their impact on the global theme, and were given the exact label and definition. 
Second: Examining the Basic and Organizing Themes: I examined the generated basic themes within each theming family for standard theming criteria. They were grouped to form organizing themes (middle-order). Each organizing theme was uniquely labeled and defined. The same rule was applied to any basic theme that did not have any relationship with other basic themes within its theming family to develop an organizing theme. Such basic themes were considered as an organizing theme that has a direct causal relationship to the global theme.

Third: Arranging the Three-Levels Themes: I followed the arrangement of three levels themes discussed in Section 3.4 as guidance or a causal path for how themes in each theming family were linked, and thus, created. However, causal relationships between themes were identified based on this arrangement and based on their contributions in causing one another within each theme's family, as well as their contributions in creating themes within the other families regardless of their level of arrangement. Table 25 - Table 29 in Appendix D: Codes to Themes Development includes and illustrates the paths of grouping (converting) the final codes into themes within each group of coding-theming families (i.e., each principle).

Since I used Atlas.ti to conduct the coding processes and then the theming process, causalities among themes were automatically updated when causally related codes were themed. For example, in the coding family of sensitivity to operations STO, each of "Contribution, Representation, and Subordination" of the workforce was uniquely labeled and defined as final codes. 
According to the literature, these three codes (activities) represent the level of the workforce participation in remaining sensitive to the operations, and they jointly causing what is called "Heedful Interrelation" (see Table 24 in Appendix C: Final Codes Labels and Definitions for definitions). Therefore, I themed these three activities into a basic theme that I labeled "Level of Employee Participation." As a result, this basic theme is becoming what is causing "Heedful Interrelation" as an organizing theme that leads to the creation of Sensitivity to Operation STO as a global theme. Figure 20 illustrates how this example case of codes was treated using Atlas.ti. Furthermore, text segments or quotations associated with the final codes were merged automatically by Atlas.ti to form one extended segment related to the developed theme. Therefore, and because of reducing overlapping codes, the third heuristic (theme overlap) was achieved. 


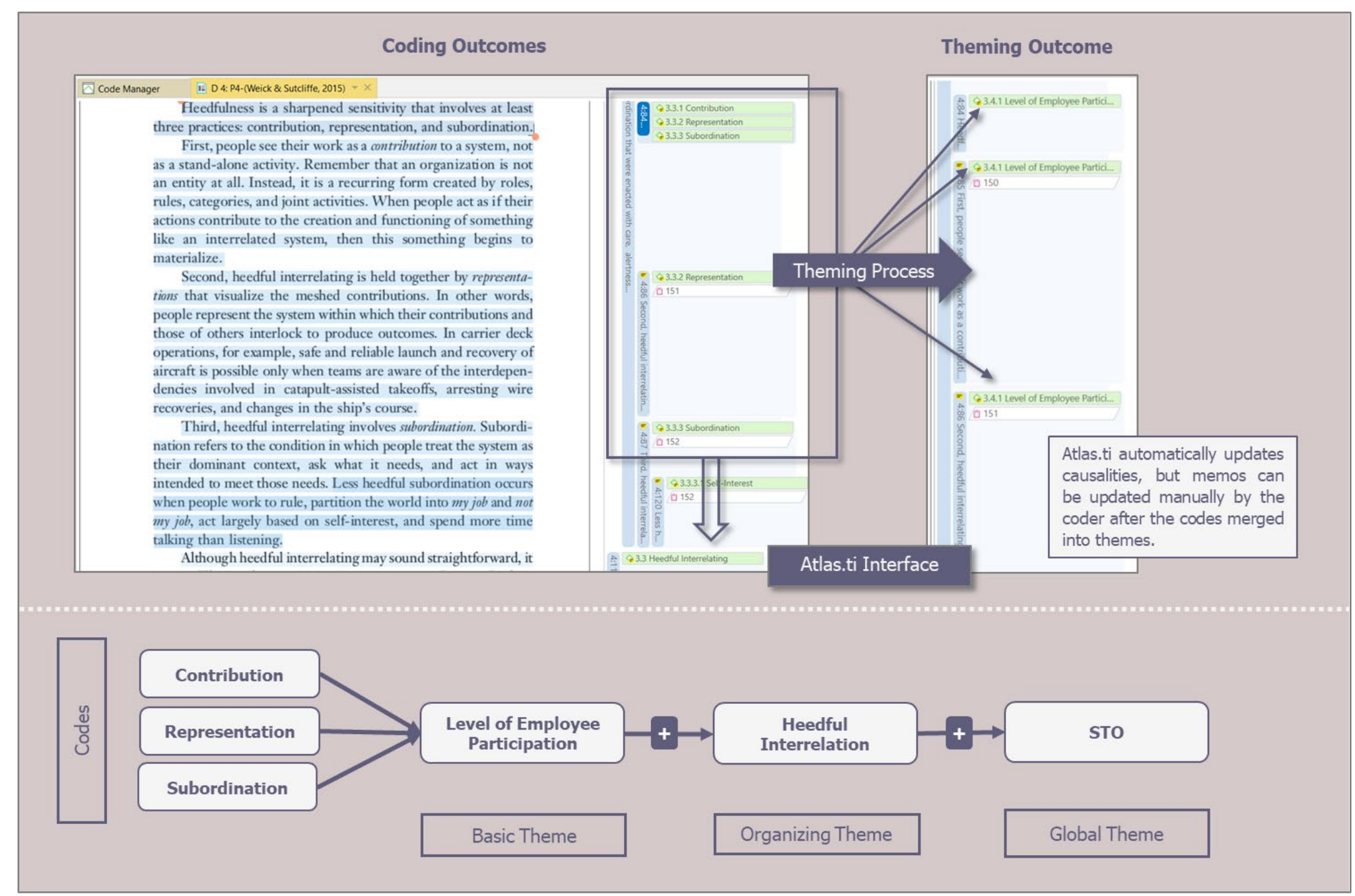

Figure 20: Illustration Example of the Theming Process 
Fourth: Developing the Thematic Network: By successively merging codes into basic themes, basic themes into organizing themes, and organizing themes into global themes, my work resulted in five thematic networks (one for each global theme) that represent the five HROT principles. Each global theme consisted of several basic and organizing themes that were frequently causally connected to basic and organizing themes in the other thematic networks. The five thematic networks were integrated into a single network that represented the HROT cognitive map. At its core was the concept of organizational reliability (OR). Figure 21 conceptualizes how the five global themes were integrated into a single HROT thematic network (or Cognitive Map). This cognitive map consists of three classes of causalities: Intra-Causalities that link themes within each network, InterCausalities that link themes within the five network, and Core-Causalities that link the five global themes to the core objective (i.e., OR). This classification is essential as it helps distinguish between the causal links among the concepts (themes on different levels) in the cognitive map and is used to quantify the causal relations in the single map.

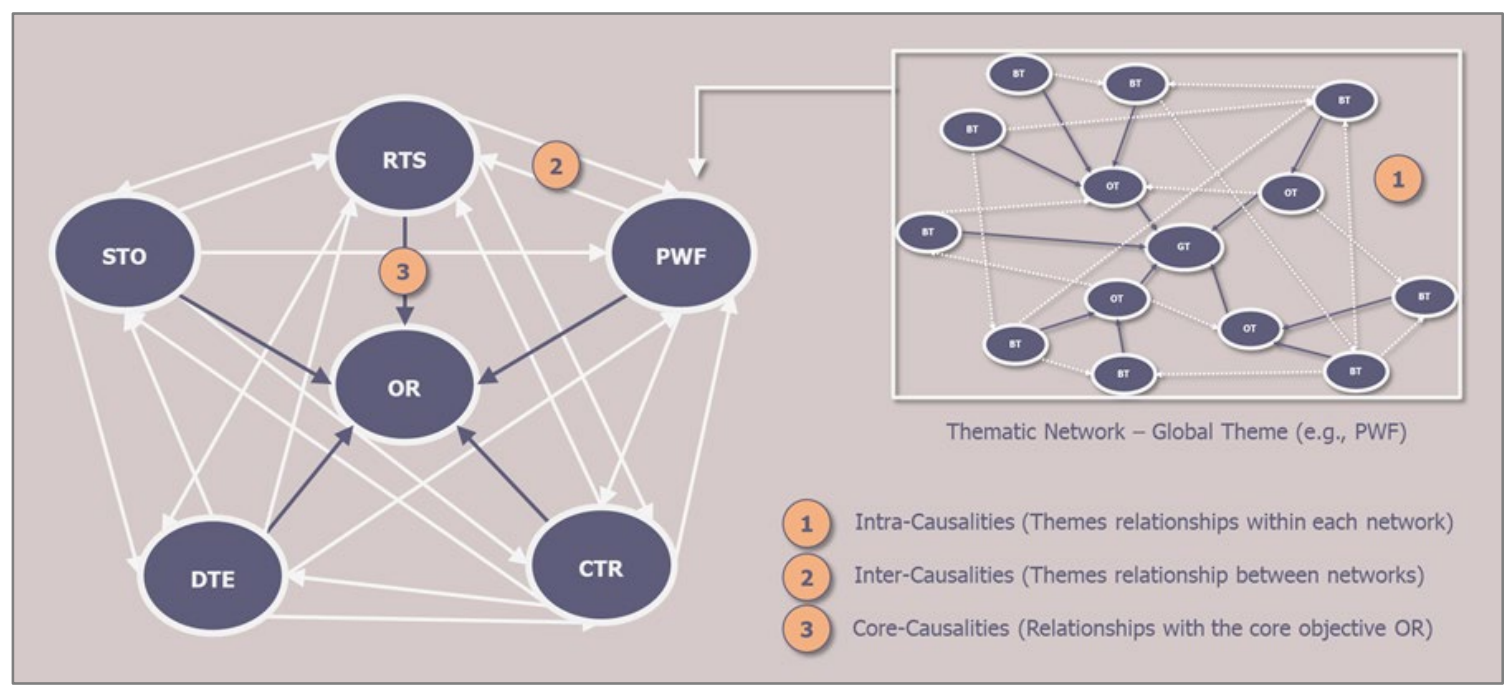

Figure 21: Conceptualization of Developing HROT Cognitive Map 
The detailed thematic structure of the first global theme: Preoccupation with Failure (PWF), is provided in Table 10. The thematic structure of PWF is provided here to demonstrate how each principle of the five HROT principles is structured and how its components (i.e., basic and organizing themes) are defined and arranged. Figure 22 depicts the thematic network of PWF, which is developed with the network tool of Atlas.ti.

The thematic structures of each of RTS, STO, CTR, and DTE are summarized in Table 11, and their detailed thematic structures are provided in Table 30 - Table 33 in Appendix E. The developed thematic networks of these four principles are depicted in Figure 48 - Figure 51, respectively, in Appendix E.

Importantly, in these thematic structures and networks, the global themes are the general objectives that needed to be maximized to maximize organizational reliability. Global themes are caused by all the organizing themes in directed relationships, and these organizing themes are caused by basic themes within the same network as well as basic themes of other networks. Moreover, each basic and organizing theme is marked with either a positive sign to infer a desirable concept in the model that needs to be maximized or a negative sign that infers an undesirable concept that needs to be minimized.

Finally, Table 12 lists the final statistics of each of the five thematic networks (global themes) and the final HROT cognitive map, including the total number of concepts (themes) in each network, basic and organizing themes, and the total number of causal connections. The single thematic network or the cognitive map based HROT five principles is created with Atlas.ti and depicted in Figure 23. 
Table 10: Thematic Structure of Preoccupation with Failure (PWF) ${ }^{19}$

\begin{tabular}{|c|c|c|c|}
\hline \multicolumn{4}{|c|}{ Preoccupation with Failure (PWF) - Global Theme } \\
\hline & Logical Reasoning $(+)$ & $\rightarrow$ & Anticipating and Controlling Latent Signals $(+)$ \\
\hline \multirow[t]{2}{*}{ 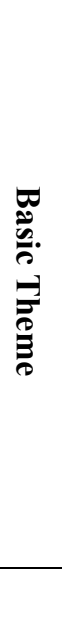 } & $\begin{array}{l}\text { Logical reasoning is thinking in a rational, sensible way. It involves, } \\
\text { in the context of preoccupation with failure, a combination of system } \\
\text { knowledge and expectation domain. This type of reasoning requires } \\
\text { understanding and knowing the physical interconnections and } \\
\text { parameters of the systems and how they work as a whole. } \\
\text { Understanding weaknesses in technologies, faults in the operations, } \\
\text { gaps in the procedure, and the orders by which errors (failures) in } \\
\text { the system are triggering each other is also imperative. This type of } \\
\text { knowledge becomes applicable when combined with the scope of } \\
\text { the system's expectations, processes, and operations. Whenever } \\
\text { something comes from outside the frame (domain), it is considered } \\
\text { an anomaly requiring an action. }\end{array}$ & 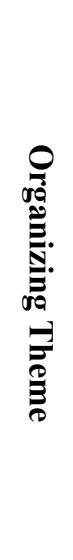 & \multirow[t]{3}{*}{$\begin{array}{l}\text { It is defined as the pattern of practicing a continual search for any } \\
\text { discrepancies and suspicious cues (anomalies), catching and resolving } \\
\text { them within a reasonable time before they grow up into serious } \\
\text { problems. An anomaly indicates something wrong that does not fit into } \\
\text { a series, or in other words, something that is a departure from common } \\
\text { order, form, or rule. An anomaly has been described as a contrast in the } \\
\text { quality of the successive segments of a stream of thoughts and serves as } \\
\text { a motive to examine intentions and expectations. Controlling latent } \\
\text { (unnoticed) signals is as important as anticipating them since those } \\
\text { signals (e.g., small errors, near misses), which accumulate, could create } \\
\text { a failure in the system. It is also known as the conversion of a cue into } \\
\text { a clue. These cues continue to evolve until a failure happens, particularly } \\
\text { when people misjudge or misinterpret their evolution. }\end{array}$} \\
\hline & Failure Knowledge and Attentiveness $(+)$ & $\rightarrow$ & \\
\hline 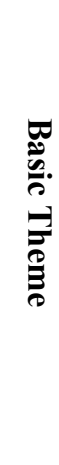 & $\begin{array}{l}\text { Capable systems that anticipate, detect, and control latent signals } \\
\text { understand that failures can evolve into the system or subsystems to } \\
\text { cause the entire system to collapse. Acquiring and sustaining failure } \\
\text { knowledge is critical to understanding the accumulations of the } \\
\text { unnoticed cues and latent signals. For the organizational property to } \\
\text { be supported, specific experience, and other knowledge types, } \\
\text { particularly system knowledge, are required. Therefore, an HRO } \\
\text { develops and maintains repositories of failure as sources for } \\
\text { information inquiry, such as experts and people with domain- } \\
\text { specific knowledge. Due to the accumulation of experience in }\end{array}$ & & \\
\hline
\end{tabular}

${ }^{19}$ The text in the table represents the contemporaneous notes of the researcher during the TA process. 


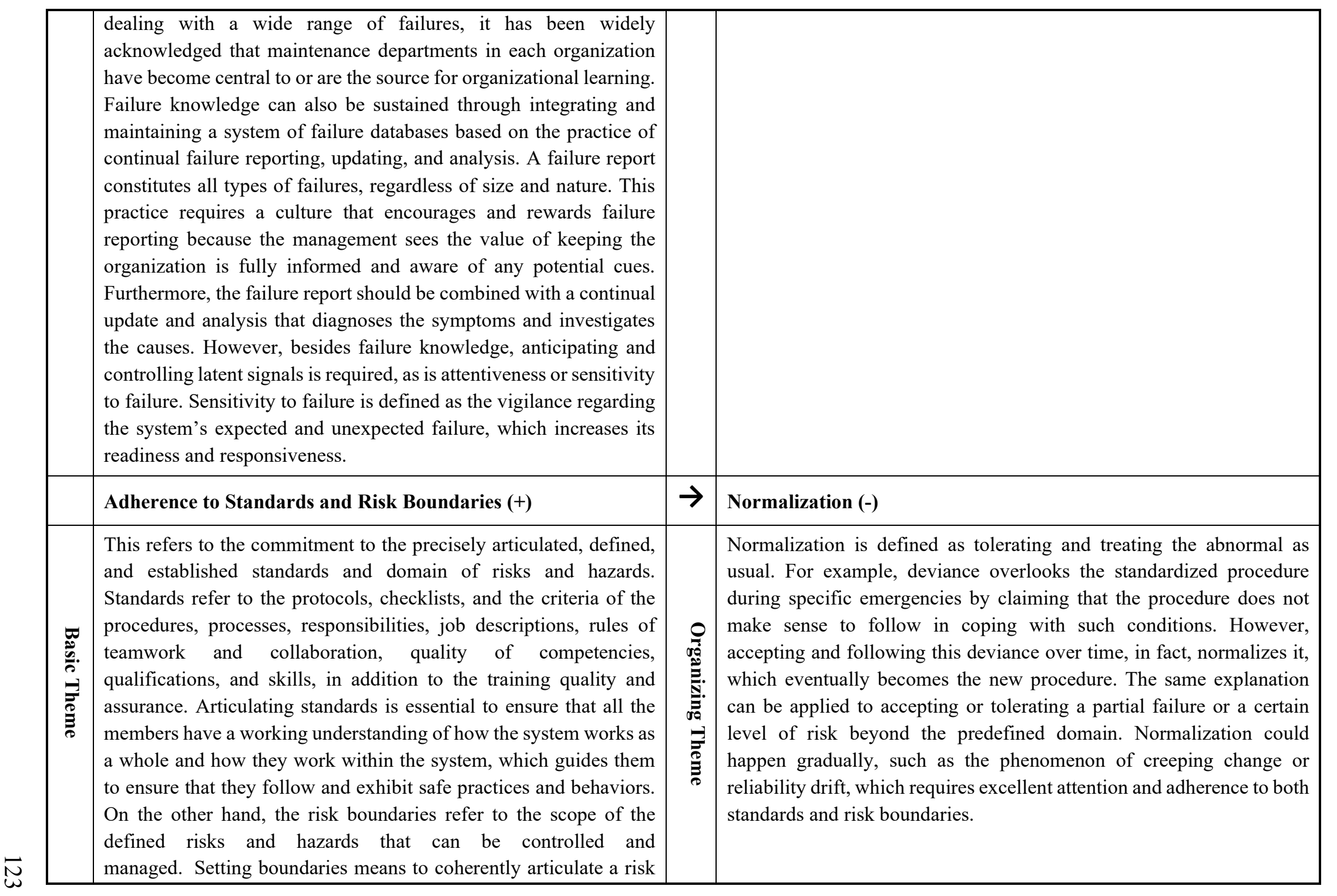




\begin{tabular}{|c|c|c|c|}
\hline & $\begin{array}{l}\text { or a hazard and what is not, why the organization may accept a } \\
\text { certain level of risk, and how it can be reduced and mitigated. } \\
\text { Overlooking the standards or accepting a certain level of risk beyond } \\
\text { the boundaries may lead to the so-called normalizing deviance, or in } \\
\text { this context, the reliability drift. }\end{array}$ & & \\
\hline & Listening to Various Voices and Inputs $(+)$ & $\rightarrow$ & Precaution as a Mind-Set (+) \\
\hline \multirow[t]{2}{*}{ 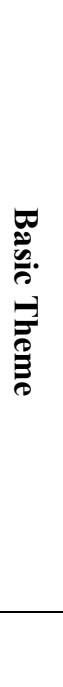 } & $\begin{array}{l}\text { To integrate a precaution mindset, an organization needs to establish } \\
\text { a culture of listening to various voices and inputs. In other words, to } \\
\text { encourage people to raise concerns and to speak up about their fears } \\
\text { regarding potential cues and allowing them to ask questions } \\
\text { regarding what is wrong or what could go wrong. This creates an } \\
\text { awareness of vulnerability, which helps in developing a course of } \\
\text { learning. Voicing concerns should be combined with calibrations } \\
\text { such as continued reporting and tracking, gaining feedback, and } \\
\text { following-up. Furthermore, a precaution as a mindset requires } \\
\text { establishing the freedom to have contradictory points of view so that } \\
\text { members of the organization are urged to criticize each other when } \\
\text { necessary. This spirit has widened the foundation of various inputs } \\
\text { that increase the variety in dealing with the unexpected. }\end{array}$ & 垔 & $\begin{array}{l}\text { Precaution as a mindset is a cultural pattern that HROs develop to } \\
\text { integrate a mindset of both wariness and doubt. Integrating wariness } \\
\text { means to embed feelings that failures can happen at any time. This is } \\
\text { very important because successful organizations become less worried } \\
\text { over time in terms of safety or reliability. Success produces } \\
\text { complacency, which may lead to underestimating reliability in the entire } \\
\text { or subsets of the organization. Precaution also requires the integration } \\
\text { of doubt as a state of mind since dealing with doubts is safer than dealing } \\
\text { with a naïve certainty. HROs adopt a doubting mindset, knowing that } \\
\text { smooth operations do not reflect reliability because dynamic processes } \\
\text { could always bring surprises and unexpected outcomes. This mindset } \\
\text { encourages a spirit of contradiction while discourages the spirit of the } \\
\text { accord. }\end{array}$ \\
\hline & Complacency (-) & $\rightarrow$ & Underestimate Reliability (-) \\
\hline 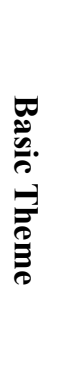 & $\begin{array}{l}\text { Complacency is a state of mind that results from experiencing } \\
\text { continued success over time. This eventually leads the organization } \\
\text { members, particularly managers, to be overly confident and highly } \\
\text { satisfied. In fact, complacency reduces people's awareness of the } \\
\text { unexpected, and it is considered a door for unforeseen events to pass } \\
\text { through and occur. }\end{array}$ & 赵 & $\begin{array}{l}\text { Underestimate reliability is a product of the incorrect certainty that } \\
\text { interim success is a true reflection of long-term reliability. When } \\
\text { success is achieved (i.e., reliability is high), organizations tend to } \\
\text { reallocate their resources or limit them (e.g., due to financial reasons; } \\
\text { reducing costs, etc.), believing there is no longer a need for such } \\
\text { resources to promote a higher level of safety, thereby changing beliefs } \\
\text { regarding reliability. Underestimating reliability minimizes the } \\
\text { preoccupation with failure and shifts the focus toward other goals. }\end{array}$ \\
\hline
\end{tabular}




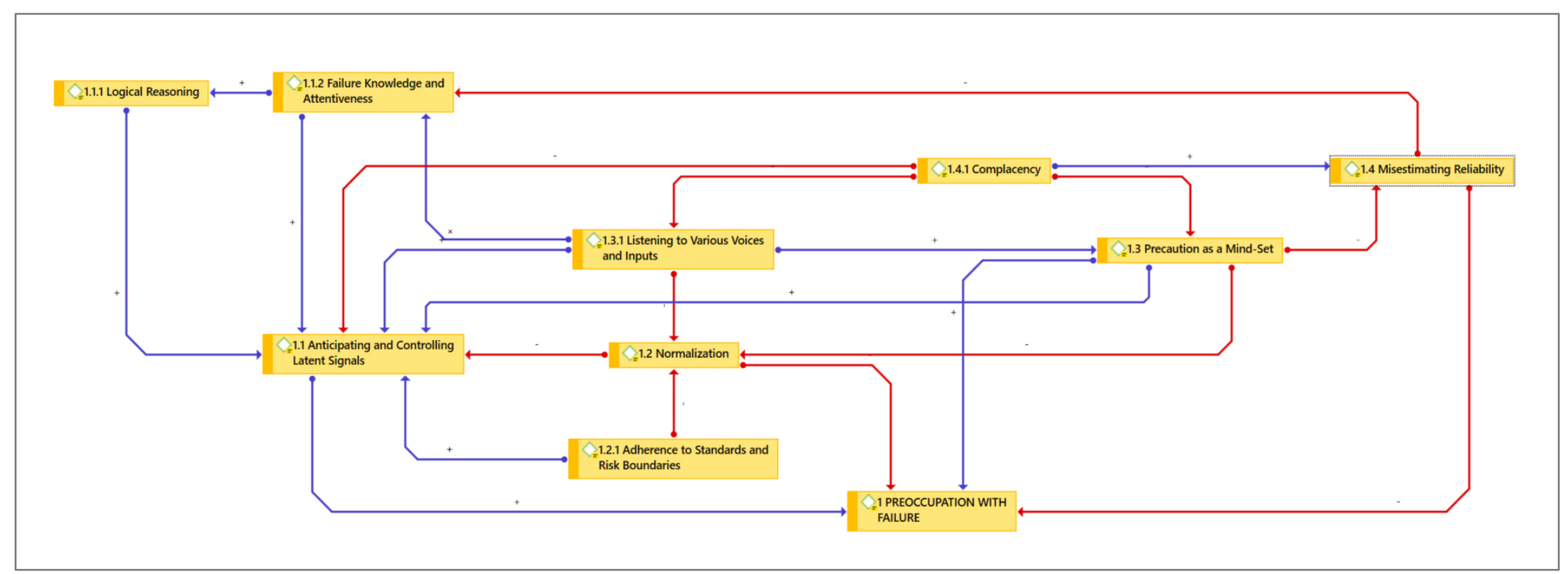

Figure 22: Thematic Network (PWF): Developed with Atlas.ti

Note: Blue lines infers positive causal relationships while red lines imply negative relationships. 
Table 11: Summary of the Thematic Structure of Each of RTS, STO, CTR, and DTE

\begin{tabular}{|c|c|c|c|c|c|c|c|}
\hline \multicolumn{2}{|c|}{ Reluctance to Simplify (RTS) } & \multicolumn{2}{|c|}{ Sensitivity to Operations (STO) } & \multicolumn{2}{|c|}{ Commitment to Resilience (CTR) } & \multicolumn{2}{|c|}{ Deference to Expertise (DTE) } \\
\hline $\begin{array}{l}\text { Basic } \\
\text { Themes }\end{array}$ & $\begin{array}{l}\text { Organizing } \\
\text { Themes }\end{array}$ & $\begin{array}{l}\text { Basic } \\
\text { Themes }\end{array}$ & $\begin{array}{l}\text { Organizing } \\
\text { Themes }\end{array}$ & $\begin{array}{l}\text { Basic } \\
\text { Themes }\end{array}$ & $\begin{array}{l}\text { Organizing } \\
\text { Themes }\end{array}$ & $\begin{array}{l}\text { Basic } \\
\text { Themes }\end{array}$ & $\begin{array}{c}\text { Organizing } \\
\text { Themes }\end{array}$ \\
\hline Distinctions & $\begin{array}{c}\text { Neglecting } \\
\text { Significant } \\
\text { Information (-) }\end{array}$ & $\begin{array}{c}\text { Loss of } \\
\text { Attention (-) }\end{array}$ & $\begin{array}{c}\text { Staying } \\
\text { Focused }(+)\end{array}$ & $\begin{array}{l}\text { Elasticity and } \\
\text { Recovery }(+)\end{array}$ & $\begin{array}{c}\text { System } \\
\text { Flexibility and } \\
\text { Adaptability }(+)\end{array}$ & & $\begin{array}{c}\text { Structure } \\
\text { Flexibility }(+)\end{array}$ \\
\hline $\begin{array}{c}\text { Requisite } \\
\text { Variety and } \\
\text { Complexity } \\
(+)\end{array}$ & $\begin{array}{c}\text { Organizational } \\
\text { Variety }(+)\end{array}$ & $\begin{array}{c}\text { Sustaining } \\
\text { Dynamic } \\
\text { Situational } \\
\text { Awareness }(+)\end{array}$ & \multirow{2}{*}{$\begin{array}{c}\text { Operations as } \\
\text { an Integrated } \\
\text { Map (+) }\end{array}$} & $\begin{array}{c}\text { Simple } \\
\text { Structure }(+)\end{array}$ & \multirow{2}{*}{$\begin{array}{c}\text { Improvisation } \\
\qquad(+)\end{array}$} & $\begin{array}{l}\text { Self-Awareness } \\
\qquad(+)\end{array}$ & $\begin{array}{l}\text { Respectful } \\
\text { Yielding }(+)\end{array}$ \\
\hline $\begin{array}{l}\text { Redundancy } \\
\qquad(+)\end{array}$ & $\begin{array}{l}\text { Organizing for } \\
\text { Sense- } \\
\text { Discrediting (+) }\end{array}$ & $\begin{array}{c}\text { Loss of } \\
\text { Situational } \\
\text { Awareness (-) }\end{array}$ & & $\begin{array}{c}\text { Personal } \\
\text { Competence and } \\
\text { Confidence }(+)\end{array}$ & & & $\begin{array}{c}\text { Available } \\
\text { Expertise }(+)\end{array}$ \\
\hline \multirow[t]{4}{*}{$\begin{array}{l}\text { Interpretation } \\
\text { and Recursive } \\
\text { Interaction }(+)\end{array}$} & $\begin{array}{l}\text { Organizing for } \\
\text { Action-Based } \\
\text { Inquiry }(+)\end{array}$ & & Pressure (-) & $\begin{array}{c}\text { Reconstituting } \\
\text { the Evolving } \\
\text { Present }(+)\end{array}$ & Sensemaking $(+)$ & $\begin{array}{c}\text { Compressed } \\
\text { Experience }(+)\end{array}$ & $\begin{array}{c}\text { Domain-Specific } \\
\text { Experience }(+)\end{array}$ \\
\hline & & $\begin{array}{c}\text { Level of } \\
\text { Employee } \\
\text { Participation (+) }\end{array}$ & \multirow{2}{*}{$\begin{array}{c}\text { Heedful } \\
\text { Interrelating }(+)\end{array}$} & $\begin{array}{l}\text { Learning from } \\
\text { Failure/ } \\
\text { Incident }(+)\end{array}$ & $\begin{array}{c}\text { Responses } \\
\text { (Actions) } \\
\text { Repertoire (+) }\end{array}$ & & $\begin{array}{c}\text { Ignoring } \\
\text { Expertise (-) }\end{array}$ \\
\hline & & Self-Interest (-) & & & & & $\begin{array}{c}\text { Overreliance on } \\
\text { Experts (-) }\end{array}$ \\
\hline & & $\begin{array}{c}\text { Interruptions } \\
\text { and Adjustments } \\
(+)\end{array}$ & $\begin{array}{l}\text { Dysfunctional } \\
\text { Momentum (-) }\end{array}$ & & & & \\
\hline
\end{tabular}


Table 12: Phase 1 Final Findings Statistics

\begin{tabular}{|c|c|c|c|c|c|c|c|}
\hline Network & $\begin{array}{c}\text { No. of Concepts } \\
\text { (Themes) }\end{array}$ & $\begin{array}{c}\text { No. of Basic } \\
\text { Themes }\end{array}$ & $\begin{array}{c}\text { No. of } \\
\text { Organizing } \\
\text { Themes }\end{array}$ & $\begin{array}{c}\text { No. of Global } \\
\text { Themes }\end{array}$ & $\begin{array}{c}\text { No. of } \\
\text { Connections }\end{array}$ & $\begin{array}{l}\text { Thematic } \\
\text { Structure }\end{array}$ & $\begin{array}{c}\text { Graphical } \\
\text { Representation }\end{array}$ \\
\hline PWF & 10 & 5 & 4 & 1 & 22 & Table 10 & Figure 22 \\
\hline RTS & 9 & 4 & 4 & 1 & 17 & Table 30 & Figure 48 \\
\hline STO & 12 & 6 & 5 & 1 & 18 & Table 31 & Figure 49 \\
\hline CTR & 10 & 4 & 5 & 1 & 13 & Table 32 & Figure 50 \\
\hline DTE & 9 & 2 & 6 & 1 & 9 & Table 33 & Figure 51 \\
\hline OR & $51 *$ & 21 & 24 & 5 & $138 * *$ & Collective & Figure 23 \\
\hline
\end{tabular}

*51 concepts (themes), including 50 themes based on the five thematic networks and one theme representing organizational reliability OR as a central concept that integrates the five networks into a single network.

**138 connections, including 79 connections of themes within networks (intra-connection), 54 connections of themes over the five networks (inter-connections), and five core-connections that connect global themes and cause organizational reliability OR as shown in Figure 21. 


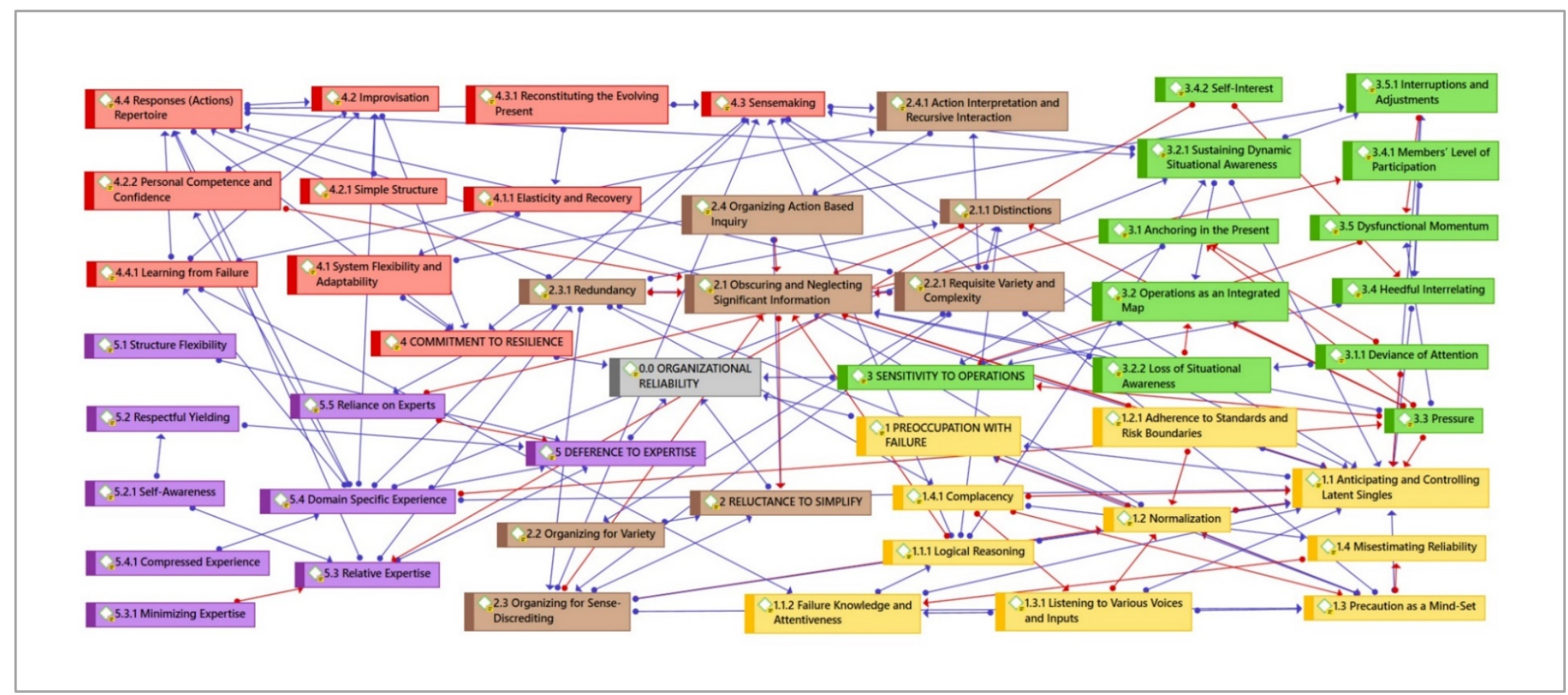

Figure 23: Thematic Network of Organizational Reliability: Developed with Atlas.ti

Note: Each global theme (i.e., principle) represents a sub-network in a different color. Organizational reliability in gray, PWF in yellow, RTS in brown, STO in green, CTR in red, and DTE in purple. 


\subsection{Phase 2: Converting the Cognitive Map (CM) into (FCM)}

In this phase, the research shifted from purely qualitative to quantitative: I quantified the strength of the causal links in the developed cognitive map and thus, converted it into an FCM. In this process, I employed thematic proximity (Armborst, 2017) to determine the causal weight, which provides an alternative to subject matter experts assigning weights based on their judgment. As discussed in Section 3.5, thematic proximity estimates the strength of the relationship between causally related themes regarding the textual content they are sharing. For the reasons discussed in Section 3.5, I used t-coefficient (eq. 11) as the numerical measure to determine causal relationships' strength. The process is described below. In total, 133 weights were determined using t-coefficient. The developed cognitive map consists of 138 causal links: five links that connect the five principles to the central concept of organizational reliability (OR), 79 links that connect themes within networks (intra-connection), and 54 links that connect themes between the five networks (interconnections). No weights were assigned between the five core concepts (i.e., the five HROT principles) and their main outcome (i.e., organizational reliability) because the literature assumes that all five principles have to be present and strongly contribute to achieving organizational reliability.

\subsubsection{Step 1: Content Filtering and Retrieving}

In this step, words within the text segments associated with each two causally related themes (source and target) are retrieved using the word list tool in Atlas.ti. The retrieved words then filtered through a filtration panel provided by Atlas.ti software to remove undesired and floating letters (e.g., a, ab, wi, etc.), numbers that are not related to the 
content (e.g., page and numbers, references, etc.), characters (e.g., $<>, \wedge, \#,+-$, , $\%, \$$, etc.) and words abbreviations that do not stand on their own to provide relevant meanings (e.g., ing, inc, rafe, etc.). The objective is to consider only words that provide concrete meanings and relevancy rather than just augmented content. I repeated this step for all the 133 causal relationships in the map (i.e., intra and inter causalities). Figure 24 is a snapshot of the Atlas.ti interface that explains how this step was conducted.

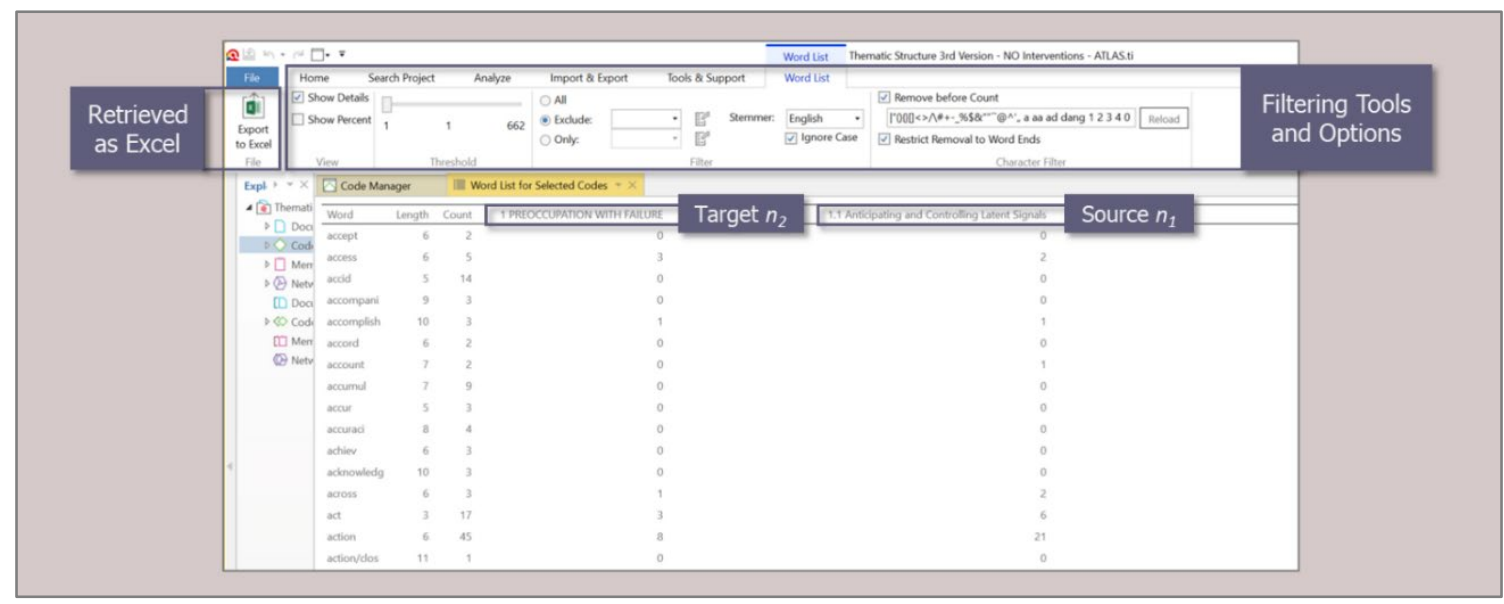

Figure 24: Example of Retrieving and Filtering Words in Textual Documents

The filtered words retrieved in Excel sheets to efficiently conduct simple mathematical calculations that determine the total number of words associated with each source $\left(n_{1}\right)$, and the total number of words associated with each target $\left(n_{2}\right)$, and then counting the shared words (intersected words) between them $\left(n_{12}\right)$.

\subsubsection{Step 2: Calculating the Weight of the Edges}

I calculated the value of the t-coefficient for each of the 133 causal links by plugging the calculated variables during the first step $\left(n_{1}, n_{2}\right.$, and $\left.n_{12}\right)$ into equation (11), which resulted in varying weights that represent the strength of the relationships between each causally related pairs of themes (see Table 34 in Appendix F). 
Importantly, t-coefficient yields values between $[0]$ and $[+1]$; however, the nature of the relationship between each two causally related themes (i.e., whether it is positive or negative) has been identified previously based on the literature during the coding and theming processes (see Figure 19 and Figure 20).

Quantifying all the causal links in the HROT cognitive map has converted it into a fuzzy cognitive map FCM. It was visualized and analyzed with mentalmodeler.com. The developed FCM based on HROT five principles is shown in Figure 25, and its preferred state and metrics are listed in Table 13.

Table 13: FCM Preferred State and Metrics

\begin{tabular}{|l|c|l|c|}
\hline Total Components & 51 & Total Connections & 138 \\
\hline Density & 0.05411 & Connections Per Component & 2.70588 \\
\hline Number of Driver Components & 10 & Number of Receiver Components & 1 \\
\hline Number of Ordinary Components & 40 & Complexity Score & 0.1 \\
\hline
\end{tabular}




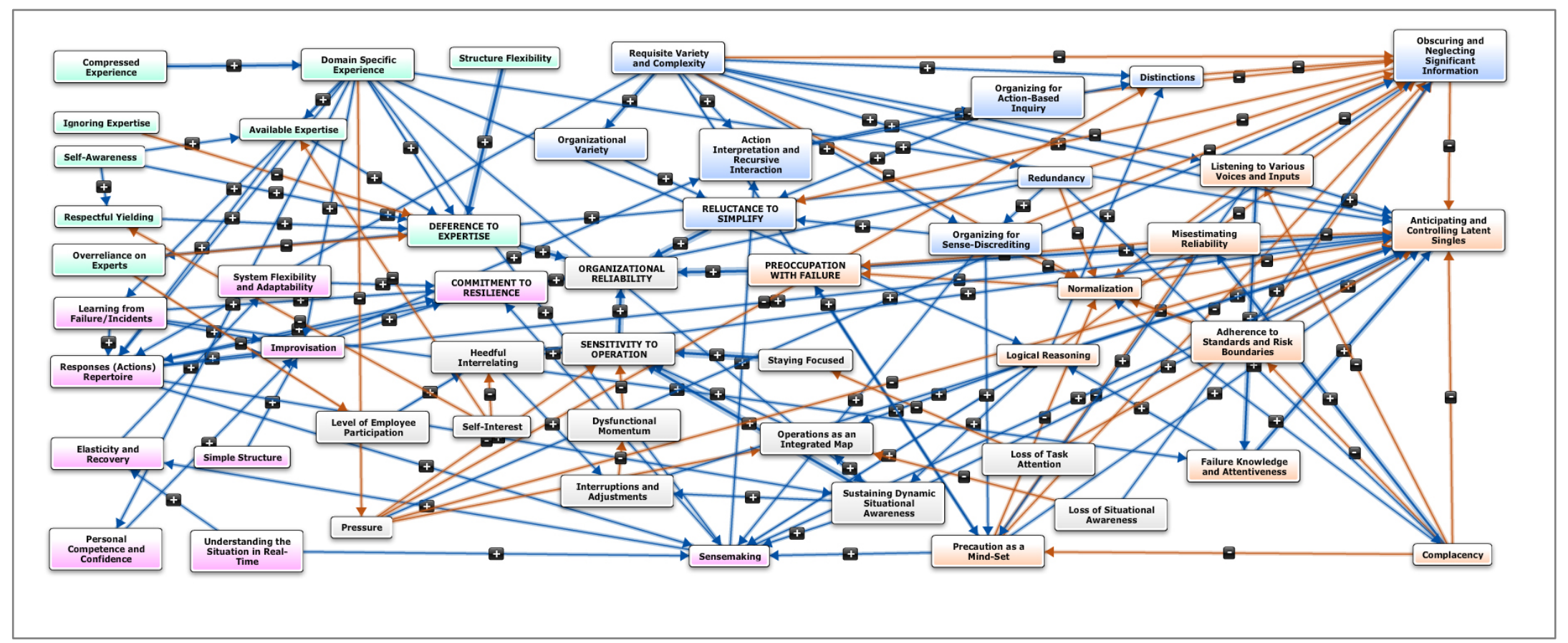

Figure 25: FCM based HROT Five Principles: Developed with Mentalmodeler.com 


\subsubsection{Step 3: Examining the Relevance of t-Coefficient}

As discussed earlier in Section 3.5, two other measures were considered as a benchmark to examine the relevancy of using the recently published t-coefficient in the context of FCM: The Jaccard similarity index or (c-coefficient) and the Cosine similarity index. Since the entire qualitative process was conducted using Atlas.ti, the values of the c-coefficient for all the 133 connections were calculated automatically by generating cross tables of themeco-occurrence Atlas.ti provides. These tables brought together each pair of causally related themes to estimate the strength of the relationship between them based on two factors: (1) the number of times each pair of themes co-occurred within the same text segments, and (2) the number of times each theme of the causally related pair was used (i.e., grounded) in the entire project. Atlas.ti, as the software, has integrated these factors into a built-in algorithm based on the Jaccard index to automatically determine the value of the ccoefficient for each causality. Furthermore, the previously retrieved and filtered lists of words in the first step were used to calculate the Cosine index of similarity using equation (9) for all the 133 causal relationships.

This process yielded two other different weights for each causal link in the map, as listed in Table 34 in Appendix F. The three weights of each edge in the model varied concerning the range between the minimum and maximum values calculated by each measure. Based on the yielded results by the three techniques and given the limitations of the c-coefficient and Cosine index discussed in Section 3.5, the following points explain why t-coefficient is a more reliable and relevant technique to quantify the weights of the edges of the HROT cognitive map, particularly with the use of thematic analysis TA as a qualitative method. 
First: Thematic analysis TA requires more engagement with the data to understand the meanings, content, and stories behind the unstructured text rather than focusing on the frequency of words and themes groundings and themes co-occurrences. Given that, neither the use of c-coefficient (focused on grounding and co-occurrence) nor the use of cosine similarity index (focused on word frequencies) was expected to yield more reliable outcomes.

Second: Changing the frequency of one word in the entire set of the words associated with either the source theme or the target theme significantly changes the calculated outcome when the cosine similarity index is used (the higher the frequency, the less the weight), while it can have a very slight to no effect on the outcome if the t-coefficient was used. This means that duplicated or similar content can dramatically change the edges' weights if the Cosine similarity index was used. However, duplicated content would not change any of the contribution factors (i.e., generated themes) and their relationships. For example, the strength of the negative relationship between "Interruption and Adjustments" as a source theme and "Dysfunctional Momentum" as a target theme was estimated as (0.482) based on the t-coefficient and (0.669) based on the Cosine index (connection no. 38 in Table 34 in Appendix F). However, if the number of one shared word in this particular case (e.g., action) is doubled (i.e., increased from 13 to 26) within the word set of either the source or the target, the strength of the relationship would change slightly to become $(0.478)$ with respect to the t-coefficient, but would be reduced to $(0.501)$ with respect to the Cosine similarity index. 
Third: If a pair of causally related themes co-occurred several times, but one theme was significantly more grounded (used) than the other, the c-coefficient would yield a minimal value regardless of the potential significance of this relationship. For example, "Misestimating Reliability" as a source theme is observed to decrease the practice of "Preoccupation With Failure" as a target, yet, the edge weight of this negative relationship based on the c-coefficient was (-0.009), which is almost zero (no relationship). The source theme was grounded only (7) times while the target was grounded (124) times within the entire selected set of text. On the contrary, the t-coefficient did not consider these factors, but rather, it considered the content that this pair of themes shared. Therefore, the strength of this relationship-based t-coefficient was (-0.364), as shown in connection number (60) in Table 34 in Appendix F.

Fourth: To compare the calculated weights of the model's edges based on the three measures, I categorized them into weak, moderate, and strong with respect to the minimum and maximum values yielded by each measure (see Table 34 in Appendix F). For example, in the t-coefficient, the calculated minimum edge weight was $(0.173)$, and the maximum was (0.945). Therefore, a weak edge weight ranged between (0.173-0.430), a moderate edge weight ranged between (0.431-0.687), and a strong edge weight ranged between (0.688-0.945). This categorization enabled defining a percentage of compatibility between the yielded edges weights by the t-coefficient with respect to the c-coefficient and Cosine index on the one hand, and the compatibility of the c-coefficient and Cosine index on the other hand. By counting the edges' weights that fell into the weak, moderate, and strong categories, the edges' weights calculated by the t-coefficient were compatible by $(80 \%)$ 
with the edges' weights calculated by the c-coefficient and by $(73 \%)$ with respect to the resulted edges' weights calculated by the Cosine similarity index. However, the results by the c-coefficient and those by the Cosine index were compatible with (59\%). Although there are no standards to compare the compatibility percentage between the three used measures, particularly t-coefficient with respect to the other two measures, the calculated compatibility percentage $(80 \%)$ and $(73 \%)$ infer a positivity regarding the relevance and the validity of the t-coefficient.

Furthermore, I plotted the three measures on the same plot shown in Figure 26, where each point on the $\mathrm{x}$-axis represents one causal relationship, and the $\mathrm{y}$-axis represented its strength. The three weights of each causal relationship for most causalities show similar patterns. However, with respect to how the low, moderate, and high weights were defined, the graph shows that the three measures have demonstrated few differences. This also infers positivity regarding the validity of t-coefficient as a reliable measure to quantify the edges of the FCM models and verifies that the appropriateness of each thematic proximity technique depends not only on the main research methodology but also on the context of eliciting the qualitative knowledge. 


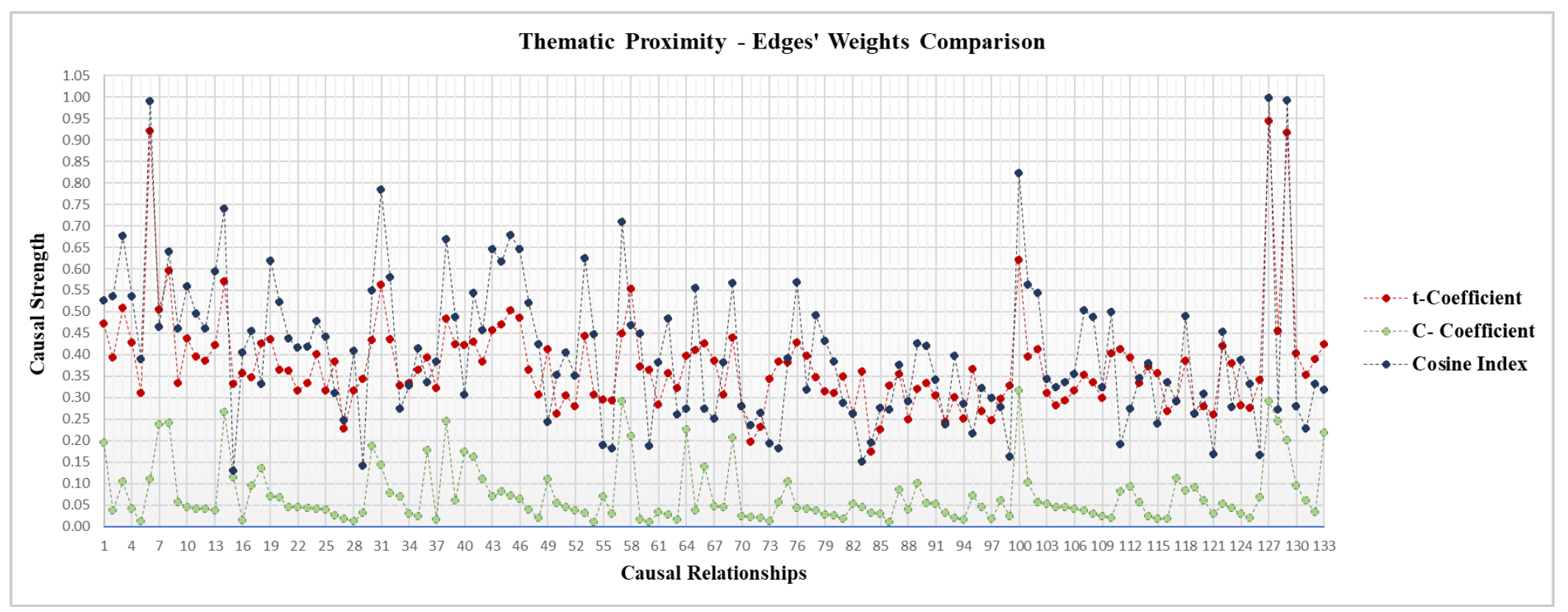

Figure 26: Edges' Weights Comparison Based on Three Techniques 


\section{Model Preliminary Application (Pilot Study)}

This chapter presents how I conducted the third research phase of this dissertation. I examined and evaluated the FCM-HROT model for feasibility and usefulness with respect to the real-world settings of the offshore oil and gas industry across a pilot study. A thorough evaluation to examine and verify the worth of the research artifact (i.e., the developed model) is highly emphasized to ensure its quality and effectiveness (Gregor \& Hevner, 2013).

A pilot study is a small-scale study usually conducted before implementing the full-scale or expanded research, which is reported on in the next chapter (Chapter 6). It guides the development of the research plans, informs, and gives feedback to the expanded research (Yin, 2017); thus, it is critical for a well-organized study design for both qualitative and quantitative research. This is particularly true when the study has a precise set of intentions and objectives to ensure methodological rigor (Ismail et al., 2018).

As shown in Figure 27, I designed this study to be consisting of an iterative process that allows model refinement in each step. In the first step, I initially tested the model for verification using couples of the model testing methods to ensure that both its structure and behaviors are initially verified before conducting the actual pilot testing. In the second step, experts from the offshore oil and gas industry were gathered in a workshop, through which: (1) I contextualized the model based on the participants' inputs, thus, evaluated it's worth based on real-world-data, and (2) I directed the research towards practicable outcomes for the industry. 


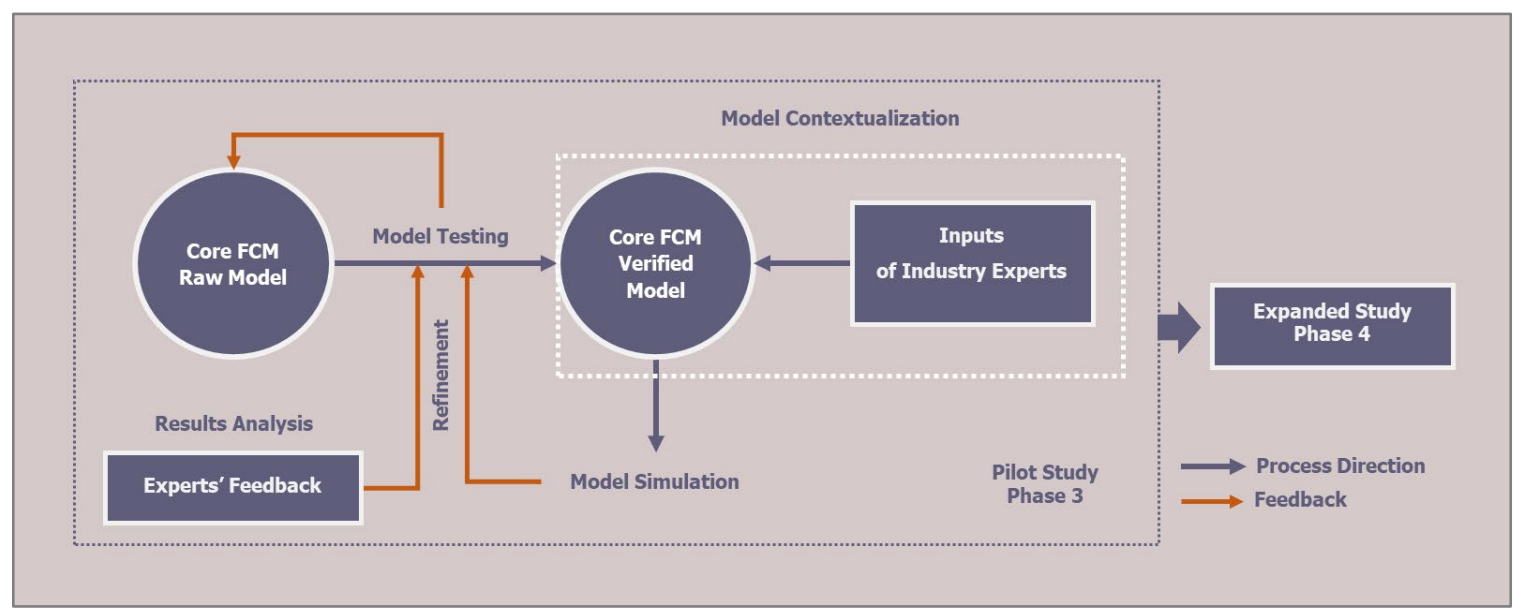

Figure 27: Pilot Study Design

The industry workshop is the core work in this pilot study since the process of evaluating the effectiveness and usefulness of the developed FCM-HROT model depends on the outcomes of the model contextualization procedure. As shown in Figure 27, the task requires the industry practitioners' total involvement to be achieved. This process's outcomes, including the experts' feedback, have been applied to the model, refined based on, and prepared for the expanded study (next chapter). The following sections discuss how I performed this pilot study.

\subsection{Model Initial Testing for Verification}

In this process, I subjected the developed FCM-HROT model to a couple of testing methods to examine its structure and behaviors based on the preliminary research assumptions. In this phase of the pilot study, tests included a structure confirmation test, extreme conditions, and sensitivity analysis. These tests were performed to prepare the model for real-world evaluation. Since extreme conditions and sensitivity analysis tests involve model simulations to be carried out, I followed "Method A" (see Table 7), 
including both "Approach A" and "Approach B." The model's structure was then refined based on the model behaviors resulted from these tests, as in the following discussions.

\subsubsection{Structure Test: Structure Confirmation Test:}

I conducted this test by comparing the structure of each thematic network individually then collectively with respect to the theoretical and empirical studies on HROT. This considers that the labels of the model concepts (themes) were given based on merging codes during the process of theming. Since these codes were labeled based on each contributing factor's meanings and definitions, conducting this test was beyond just comparing the concepts' labels. This test is more of a comprehensive review and a comparison that resulted in a verified structure. The process involved examining the concepts and the relationships among them using the tool of the co-occurrence tables and co-occurrence explorer of Atlas.ti as guidance to review and inspect the model structure. However, this test might have encountered subjectivity, which I coped with by subjecting the model to extreme conditions as a structure-oriented-behavior test and sensitivity analysis as a behavior test. Both tests are discussed as follows.

\subsubsection{Behaviors Tests: Extreme Conditions and Sensitivity Analysis Tests}

Conducting these tests involved simulating the FCM-HROT model based on the simulation approaches in Method A (see Table 7). The simulation process has been conducted using the Python FCM package developed by Aminpour (2018), as follows.

First: I performed these tests following both "Approach $A$ " and "Approach B" by activating a state vector with different activation levels for single concepts at a time and then examined the performance of the HROT five principles based on these varying inputs. 
Since the five HROT principles are well-described in the literature, the system's behaviors were clearly predicted.

In this process, twenty concepts (themes) of the model were individually activated (100\% turned on) and subjected to extreme conditions to analyze the model for sensitivity. In two cases, namely "Ignoring Expertise" and "Self-Awareness," the model showed unexpected behaviors, which indicated that some discrepancies exist in its structure. Therefore, I examined the structure by tracing back every assumption that was made during the entire creation process of the model. This action necessitated examining all the steps in the process, starting with the original text, where all research arguments and suggestions concerning these cases were investigated. Indeed, there were wrong assumptions that required an essential calibration of the model's structure. Thus, I modified it accordingly and re-calculated the t-coefficient for all the concepts in question. After this modification, the model behaved as expected. Figure 28 presents the HROT principles' state before and after modifying the model structure for these two cases.

Second: I tested the model using "Approach C" of "Method A" to determine the behaviors with respect to the variations in the concept values by randomly varying up to five concepts in the input vector at a time to examine the range of the possible outcomes for all five HROT principles. The model did not show any abnormal behaviors and performed as expected. Figure 29 presents two different cases, as shown in Graph A and Graph B, which has given expected behaviors of interest concepts.

In the first case as in Graph A, it is evident that many cases when preoccupation with failure PWF showed a high degree, each of sensitivity to operations STO, commitment to 
resilience CTR, and reluctant to simplify RTS have demonstrated high degrees as well, yet, deference to expertise DTE has been in the middle. However, in the other case, as in Graph B, when many cases have maximized the degree of deference to expertise DTE, the degree of PWF has been degraded. Both cases have shown behaviors like those of real-world behaviors, as the literature on HROT has described, which confirmed that in many cases, various efforts would not lead to achieving a similar degree of change for all the principles at the same time.

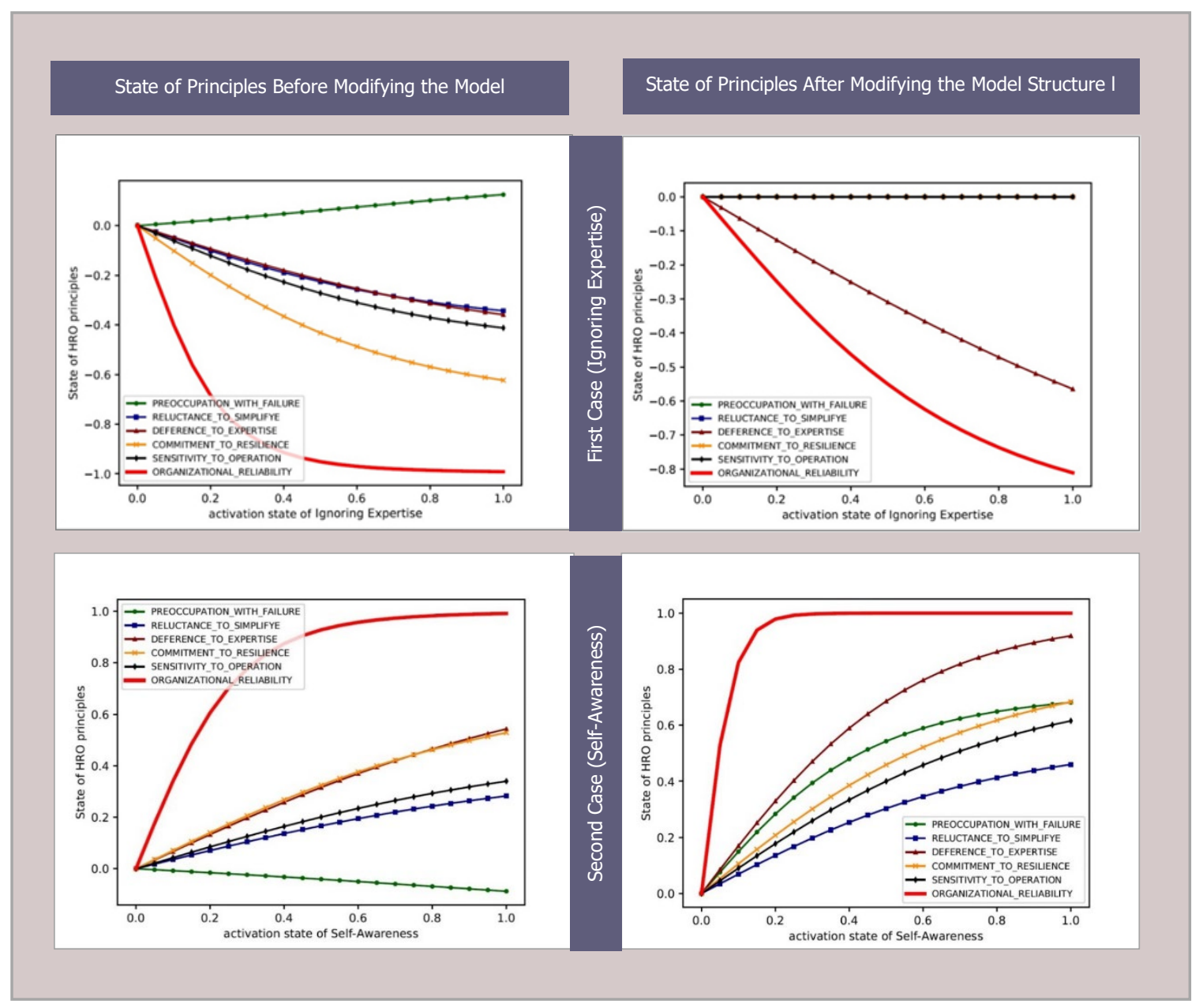

Figure 28: Sensitivity Analysis Test Before and After Modifying the Model Structure 


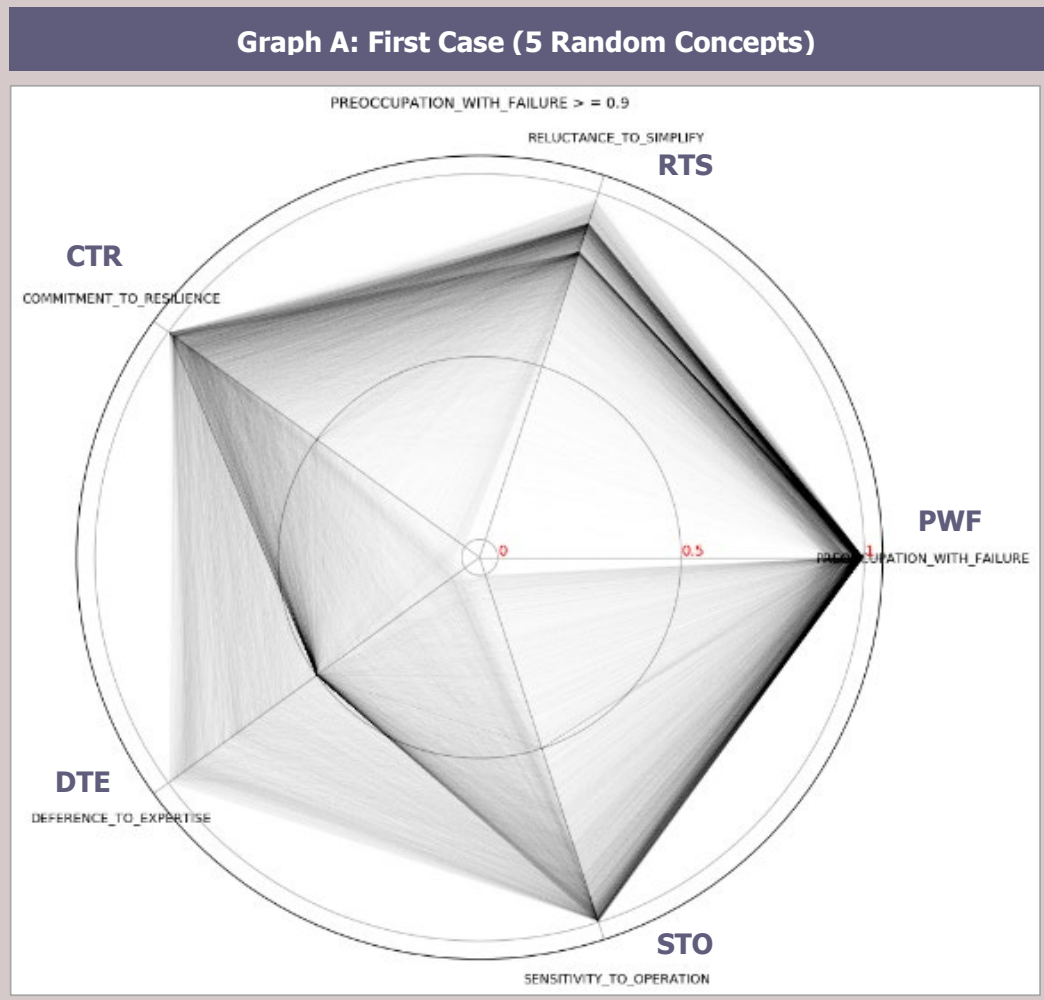

Graph B: Second Case (5 Random Concepts)

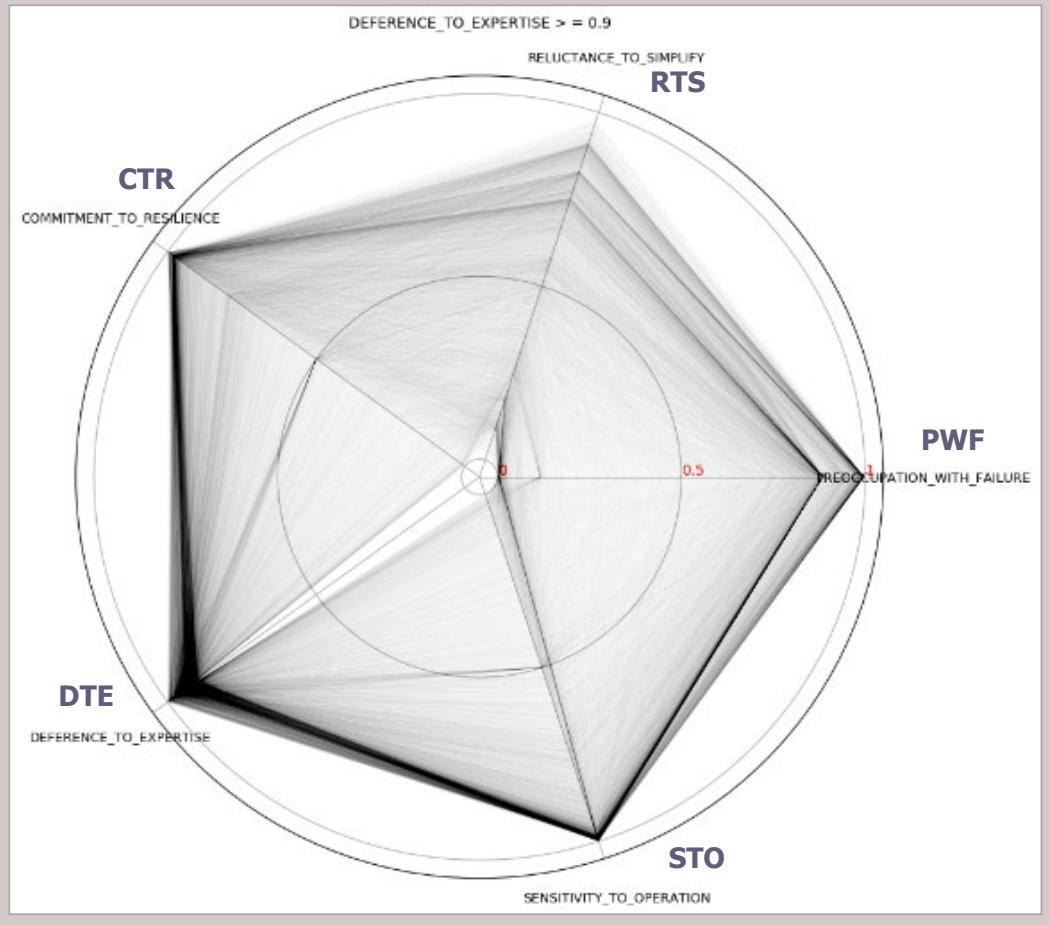

Figure 29: Examples of Model Testing for Verifications (Uncertainty Analysis) 


\subsection{Model Contextualization Procedure}

The FCM model generated and tested in the earlier steps represents a generalized HROT dynamics model, based on research done in many organizational contexts. It is, independent of industry, the core of organizational reliability. However, as discussed above, managerial interventions (e.g., training programs, leadership practices, incentive structures) do not work equally well in each industry context. To assess and plan managerial interventions for offshore oil and gas, the general model needs to be contextualized to the industry's specifics settings. This occurs by identifying the relationship between managerial interventions and elements of the core model. The contextualization permits the simulation of managerial interventions under various scenarios that consider different combinations of model variables to represent internal factors (under the company's control, such as its incident reporting procedures) and external factors (in the business environment, such as new government rules and regulations).

Model simulations can be used to determine the varying degree of effectiveness of different interventions under different conditions. Notably, the simulation considers all model elements concurrently. Thus, it can identify if a planned intervention with positive impacts on some of the five HROT principles has unintended negative consequences for other HROT principles. This information can be used to improve interventions by avoiding or reducing negative impacts and selecting an array of interventions that are likely to enhance organizational reliability. Scenario planning and model simulation techniques can thus be 
used to determine the system's possible future state and uncover gaps and weaknesses in the safety system.

To contextualize the model for offshore oil and gas, I first selected and defined industryrelevant managerial interventions that are either already used in the industry or debated as something the industry should use. I then had to determine how these interventions can be, in principle, represented in the core model and how industry experts could do so in practice. Figure 30 graphically illustrates the model contextualization procedure through which industry experts participate in modeling the selected managerial interventions by establishing causal relationships with the concepts in the outer layers of the model, or other words, HRO-relevant concepts. Experts also specify the nature of the established relationships and assig their strength; then, the model is simulated by selecting initial state vectors to activate each intervention individually or as groups of interventions. The outcomes of this process would be visualized to reflect the responses of the concepts of interest (i.e., the five HROT principles) to the applied interventions. These steps cumulated in the preparation and execution of a workshop with industry practitioners. Each of these steps is described below and is used in both this pilot study and the expanded study in the next chapter. 


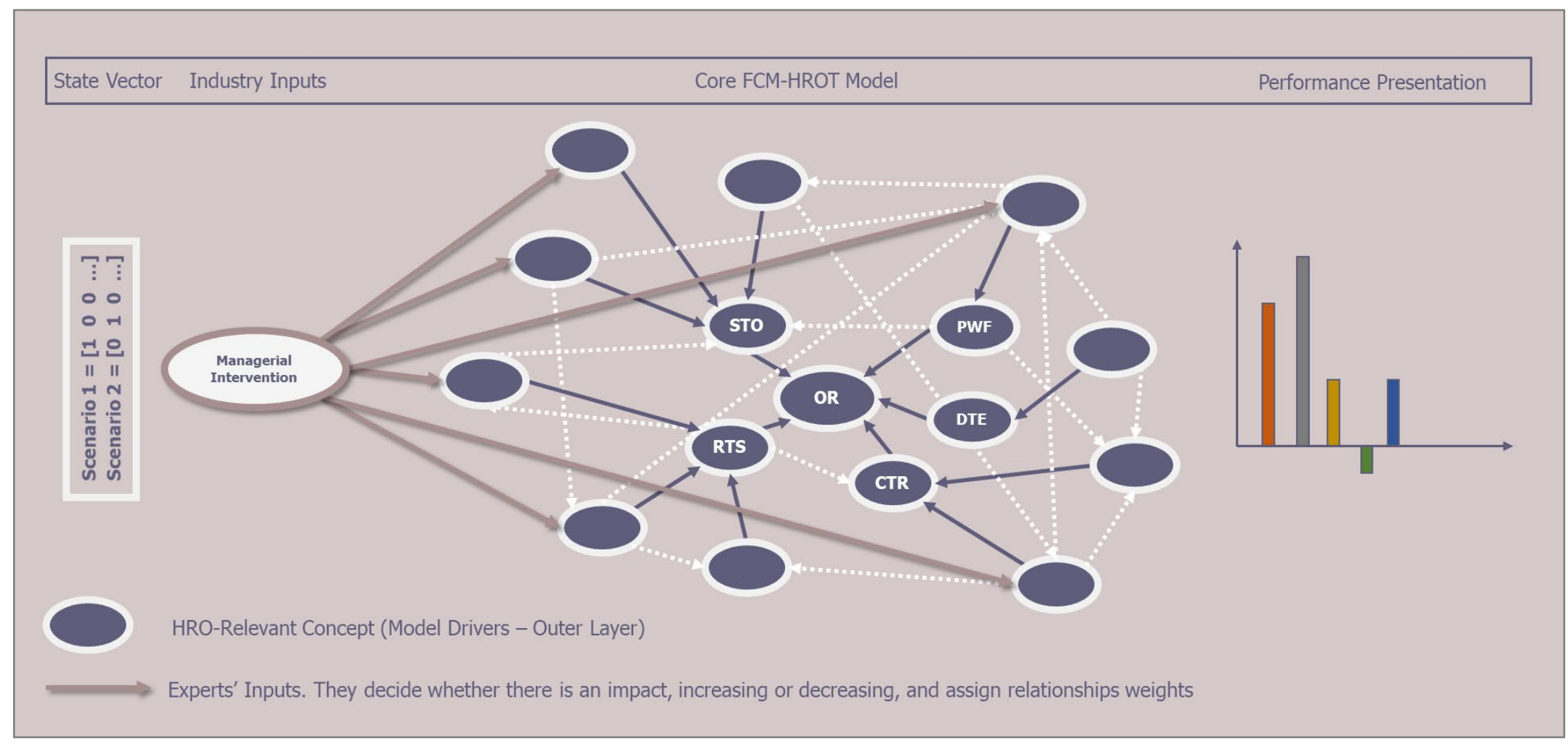

Figure 30: Graphical Representation of the Process of Model Contextualization 


\subsubsection{Selecting Relevant Managerial Interventions}

A managerial intervention is an action taken by the management to implement arranged policies or procedures such as making a change in people's beliefs and behaviors or enhancing their competencies and skills. This is usually necessary, particularly regarding safety-related practices. There are many interventions that organizations can apply to improve their safety-related practices; however, successful implementation of an intervention depends on the industrial context and its operational settings. For example, fostering social ties based on mutual respect may work well for a specific healthcare industry organization. Yet, it may not work at all for organizations in the offshore oil and gas industry.

High reliability organizations usually design and implement interventions that can be critical to the limited use of trial and error. Interventions, particularly those designed to enhance safety performance in high hazard organizations, often are costly, and chances that such interventions can make noticeable changes are not guaranteed; therefore, the selection of interventions can be very challenging.

I selected a list of thirteen managerial interventions that have been discussed within the literature of both high reliability organizations theory and the offshore oil and gas industry. These managerial interventions (see Table 14) are expected to provide adequate inputs, through which the model is simulated to examine the system's behaviors. 
Table 14: Selected Managerial Interventions

\begin{tabular}{|c|c|}
\hline Intervention & Definition and Description \\
\hline Stop-Work-Authority & $\begin{array}{l}\text { Employees and contractors are trained to stop working on an activity if, in their opinion, a continuation of the work poses a } \\
\text { threat to human health, property, or the environment. This extends to stop-work cases that are not covered by government or } \\
\text { company regulations. People are celebrated for stopping work, even if service is disrupted. Encouragement of stop-work } \\
\text { authority is particularly emphasized when rank (line workers, contractors) or conditions (layoffs and economic worries, high } \\
\text { production pressure) make it likely that people fail to stop work. }\end{array}$ \\
\hline $\begin{array}{l}\text { Reporting of Near Misses } \\
\text { and Safety-Critical } \\
\text { Events }\end{array}$ & $\begin{array}{l}\text { Management actively encourages the reporting of near misses and safety-critical events while reducing barriers faced when } \\
\text { reporting. Possible approaches: (1) clear definitions of what constitutes a reportable incident, (2) supervisor sets example } \\
\text { (i.e., supervisors report themselves), (3) reporting as an expectation in performance evaluation, (4) immediate positive } \\
\text { feedback for the reporting employee, pointing out the problem that may have been avoided as a result of him/her taking } \\
\text { action, (5) no blame or fear of punishment for the reporting employee, (6) if the level of reporting is generally low: honorable } \\
\text { mention or other visible (but not financially significant) rewards for units which are top reporters, (7) After correct action is } \\
\text { taken, inform the reporting employee about it, (8) where appropriate, publicize the report and the corrective action beyond } \\
\text { the immediately affected organizational unit. }\end{array}$ \\
\hline $\begin{array}{l}\text { Foster Social Ties and } \\
\text { Mutual Respect }\end{array}$ & $\begin{array}{l}\text { It is creating a work environment that fosters social ties building on mutual respect. Actions can include mentoring new team } \\
\text { members, creating opportunities for social interactions, and setting clear expectations for respectful behavior. This addresses } \\
\text { reliability challenges that occur when people do not know each other well enough, do not interact frequently, have modest } \\
\text { mutual knowledge but move from one area or role to another, or do not collaborate over extended periods. }\end{array}$ \\
\hline $\begin{array}{l}\text { Foster a Sense of } \\
\text { Personal Accountability } \\
\text { for Safety on All Levels }\end{array}$ & $\begin{array}{l}\text { The assessment processes of safety involve the employees on all levels. Everyone takes personal responsibility for the process } \\
\text { and personal safety, as well as environmental oversight. Personal accountability requires self-assessment and self-reflection } \\
\text { and competencies in identifying hazards and improving the work processes. }\end{array}$ \\
\hline
\end{tabular}




\begin{tabular}{|c|c|}
\hline $\begin{array}{l}\text { Implementing Safety } \\
\text { Huddles }\end{array}$ & $\begin{array}{l}\text { Gather employees from different levels for a daily short face-to-face meeting. The meeting is designed to give frontline staff } \\
\text { and others in the system prospects to stay informed, assess events, make and share plans for safeguarding well-organized } \\
\text { operations. This allows them to make sense of any situation, errors, and worries and to review options for resolving issues or } \\
\text { reducing them in the future. Safety huddles can also be done via phone or video conferencing or in-person with varying times } \\
\text { of implementation. Some organizations do multiple huddles a day (e.g., at every shift change or once in the morning and } \\
\text { once in the afternoon). }\end{array}$ \\
\hline $\begin{array}{l}\text { Practicing Tabletop } \\
\text { Exercises }\end{array}$ & $\begin{array}{l}\text { Scenario-based training that brings participants around a table to assess and reflect on different features of preplanned } \\
\text { scenarios. Participants simulate the scenario in their minds, through discussion, without running the exercise in real life. They } \\
\text { discuss the scenario experience face-to-face and share their responses. This allows them to understand roles and } \\
\text { responsibilities and solutions to different situations. }\end{array}$ \\
\hline $\begin{array}{l}\text { Practicing Post-Event } \\
\text { Debriefings }\end{array}$ & $\begin{array}{l}\text { Meetings held after an event (a real-world incident or a simulation/exercise) to discuss, summarize, and learn from the } \\
\text { activities the team has just experienced. Debriefing can be arranged for different events and focused on failures but also } \\
\text { successes. De-briefing can help employees to manage feelings and concerns about the event they went through. }\end{array}$ \\
\hline $\begin{array}{l}\text { Practicing Emergency } \\
\text { Drills }\end{array}$ & $\begin{array}{l}\text { Emergency drills are extensively realistic (often physical) simulations of significant accidents or hazard-scenarios and safety } \\
\text { action plans for responding to such events. Drills are implemented at different levels of the organization and may encompass } \\
\text { the entire emergency organization. Observers are appointed to closely follow the drill and summarize the drill's problems } \\
\text { and accomplishments in an evaluation report. }\end{array}$ \\
\hline $\begin{array}{l}\text { Institutionalizing } \\
\text { Prosocial Motivation }\end{array}$ & $\begin{array}{l}\text { People's desire to expand efforts that benefit others because they empathize with them or think of them as someone they like, } \\
\text { want to be loyal to, or are responsible for. Prosocial motivation can be increased by orienting individuals toward others and, } \\
\text { for example, pointing out how individual choices relating to safety impact others. }\end{array}$ \\
\hline $\begin{array}{l}\text { Institutionalizing } \\
\text { Emotional Ambivalence }\end{array}$ & $\begin{array}{l}\text { It describes a mixed feeling, such as feeling confident and cautious, or optimistic, and alerting what could go wrong. This is } \\
\text { often described as having doubts and hope. Having such mixed feelings makes people more open to alternative perspectives, } \\
\text { improving their ability to anticipate failures and effectively respond to the unexpected. Managers can exploit this by providing }\end{array}$ \\
\hline
\end{tabular}




\begin{tabular}{|l|l|}
\hline & $\begin{array}{l}\text { the missing view or considering it in task assignments (e.g., given meaningful and challenging tasks that need to be done } \\
\text { carefully and without overconfidence to less experienced employees). }\end{array}$ \\
\hline $\begin{array}{l}\text { Managing Reliability } \\
\text { Drift }\end{array}$ & $\begin{array}{l}\text { People and organizations form habits that often cause processes that operate according to standards to degrade over time and } \\
\text { drift towards lower reliability levels. This can result in a slow and steady uncoupling of practices from the formally written } \\
\text { procedures. This can be managed through continuous monitoring, control, and acting against the drift. }\end{array}$ \\
\hline Mracticing Individual & $\begin{array}{l}\text { Mindfulness is a mental state accomplished by concentrating one's perception of the present moment while calmly } \\
\text { acknowledging and accepting one's feelings and thoughts. It can be trained and practiced, e.g., by kicking off work with a } \\
\text { short meditation/breathing exercise, taking conscious short breaks between tasks, and inviting short (self-) reflections. }\end{array}$ \\
\hline $\begin{array}{l}\text { Implementing } \\
\text { Just-In-Time Learning }\end{array}$ & $\begin{array}{l}\text { They are creating learning opportunities that employees can access at the point and time of need when they encounter a } \\
\text { critical situation. This can be a new task for them, a technical problem, or a safety incident. In these situations, learners are } \\
\text { highly motivated to learn and to implement their new knowledge immediately. Access is facilitated 24/7 through } \\
\text { communication technologies (e.g., phone apps). Learning is facilitated through prepared content (e.g., manuals, training } \\
\text { videos) and access to colleagues and experts. The learner can submit content about incidents, causes, and solutions, thus } \\
\text { contributing to the knowledge base. }\end{array}$ \\
\hline
\end{tabular}




\subsubsection{Applying Interventions to the Core Model}

According to the definition of the selected interventions and how they potentially influence specific HRO-relevant concepts, I developed an approach to apply these interventions to the core model through the several driver concepts on its boundary (i.e., core model exogenous concepts). The established relationships between each intervention and the concepts are either increasing relationships with a strength of [+1] or decreasing relationships with a strength of [-1]. These relationships and their nature (i.e., increasing or decreasing) have been extracted from the literature, while their weights were suggested to be [+1] for positive and [-1] for negative as default strength. However, industry practitioners decide whether there is a relationship, its nature, and weight in the real-world contextualization process. Table 15 is an example of how these relationships are established and provided to the industry practitioners during the contextualization procedures. The other twelve interventions and the list of the corresponding HRO-relevant concepts are provided in Table 35 in Appendix G.

Table 15: Example of How Scenarios Based Interventions Established

\begin{tabular}{|l|c|l|}
\hline Intervention (Source) & $\rightarrow$ & HRO-Relevant Model Concepts (Target) \\
\hline \multirow{5}{*}{ Stop-Work-Authority } & + & Adherence to Standards and Risk Boundaries \\
\cline { 2 - 4 } & - & Dysfunctional Momentum \\
\cline { 2 - 3 } & - & Pressure \\
\cline { 2 - 3 } & + & Level of Employee Participation \\
\cline { 2 - 3 } & + & Personal Competence and Confidence \\
\cline { 2 - 3 } & + & Simple Structure \\
\cline { 2 - 3 } & + & Structural Flexibility \\
\cline { 2 - 3 } & + & System Flexibility and Adaptability \\
\hline
\end{tabular}




\subsection{Industry Workshop}

The underlying research and the process of building the FCM-HROT were presented in April 2018 at an industry workshop, which has been hosted by the Center for Offshore Safety (COS) in Houston, TX. This workshop was a part of a funded project to strengthen the offshore oil and gas industry's safety culture and has been facilitated by a team of three senior researchers and three doctoral students, including myself. This workshop was the core task of conducting the pilot study. I preliminary evaluated the feasibility and usefulness of the created model with respect to industry experts' opinions and based on real-world context.

The workshop has been attended by twenty-two participants with various operational and safety-related responsibilities, including safety managers, safety auditors, safety engineers, HSE managers, and safety trainers, and influencers. Besides COS representatives, participants attended from pioneering and leading oil and gas companies such as BP, Shell, ExxonMobil, Chevron, Rowan Companies, Subsea7, and BHP.

The workshop lasted for three hours and a half. It included one hour for project presentation and exercise descriptions, 45 minutes to conduct exercises by participants, 30 minutes break, 45 minutes reporting practices, and 30 minutes for general discussions. The following section discusses the preparations for the workshop and data collection.

\subsubsection{Preparing the Workshop}

This workshop required a certain level of arrangements, included communications with COS to host the workshop, and decisions regarding the time, date, and place to where it 
has been held. With the assistantship of COS, participants from the mentioned major oil and gas companies have been invited to attend the workshop at the COS location. I prepared the required material, including brochures and handouts that have been distributed to the participants, comprising the definitions of each managerial intervention and the suggested list of the corresponding HRO-relevant concepts and instructions on how to conduct the exercise. The research team assisted with examining these materials for readability, clarity, and information coherence, as well as the preparation regarding the agenda, plans, task assignments, and activities to collect the data during the workshop.

\subsubsection{Data Collection}

As mentioned earlier, this workshop meant to collect data from industry experts based on their field experience so that the model is contextualized with respect to the research context. The workshop participants were grouped into three breakout teams and provided with previously prepared handouts (see Appendix H) that included a set of defined interventions and lists of corresponding defined HRO-relevant concepts (the first three interventions in Table 14). Each team was assigned with one intervention and was asked to do the following:

1. Review the provided definitions of the intervention and the corresponding list of the HRO-relevant concepts.

2. Deliberate to decide whether the examined intervention increases, decreases, or has no influence on any of the concepts in the provided list (i.e., no established relationship).

3. Assign a weight to each established relationship based on a scale of three points: Slight $(0.33)$, moderate $(0.66)$, and extreme (0.99). 
Each team spent around 45 minutes to conduct this exercise and 30 minutes to report their decisions to the other teams. Teams' inputs, comments, and suggestions were collected and documented by facilitators from the researchers' team.

\subsubsection{Model Simulation and Behaviors}

During the workshop, and to show the participants how their decision-making process has influenced the five HROT principles' behaviors, we applied the collected inputs to the model, as illustrated in Figure 30. Using mentalmodeler.org, adjacency matrices were generated and then entered a Python FCM package to simulate this model. Since the strength of all the relationships provided by the practitioners were exact values (no variation as they reached agreements), therefore, the model has been simulated following “Approach A \& Approach B" of “Method B” (see Table 7), through which, we selected a state vector to activate each intervention individually, and then collectively. Using hyperbolic tangent (eq. No. 8) as a transferring function with a Lambda value of (2), we simulated the model to show sensitivity analysis and bar graphs that presented the simulation outcomes, which illustrated the effects of their opinions on the concepts of interest and for all the concepts in the core model.

The participants found that the generated results were remarkable, particularly with respect to the cases where their inputs did not match the research expectations regarding the nature and the scale of the interventions' impacts. However, simulating the model based on the three examined interventions has presented behaviors similar to the industry system's behaviors as expected. The following cases demonstrate significant observations based on this pilot study. 
Case 1: Practitioners make decisions based on real-world operations to avoid conflicts with their strategies and interventions. This was observed in some of the decisions made by Team 1 and Team 3. In the exercise, both teams found that the suggested positive impacts of the interventions on a few model concepts contradicted their strategies. These two cases are marked in orange color in Table 36 and Table 38 in Appendix H. However, these cases resulted in different outcomes depending on the centrality of the affected concepts, feedback loops, and the connection strength with other core model concepts.

The change made by Team 1 led to a slightly negative influence on the performance of the directly linked principle (CTR), as shown in Figure 52 in Appendix H. In contrast, the change made by Team 3 led to a highly negative impact on the directly linked principle (RTS). Even higher adverse effects on the indirectly linked principle (PWF), as shown in Figure 54 in Appendix H . Both teams justified their decisions based on their real-world strategies. For example, Team 3 urged that Intervention 3 "fostering social ties based on mutual respect" found to be contradicting with a mentoring program that they recently implemented in one of the organizations since this intervention increases "action-based inquiry," which increases the dependability of some employees to enquire information from experts rather than their assigned mentors.

Case 2: Designing and implementing an intervention does not necessarily improve or negatively influence the five principles all together at the same time. This outcome was observed by applying Intervention 2, which showed no influence on the fifth principle (DTE), as shown in Figure 53 in Appendix H. In real-world operations, the five principles 
cannot be maximized simultaneously, but managers can make trade-offs to enhance the overall performance.

Case 3: For every intervention, there can be some undesired side effects, which can be reduced by designing and implementing other interventions. Integrating both Intervention 2 and intervention 3 at the same time has led to acceptable performance of the five principles by mitigating the side effects of Intervention 3 discussed in Case 1 above. Figure 55 in Appendix H presents these observations.

\subsection{Summary of the Results}

The resulted outcomes of simulating the developed FCM-HROT model with respect to real-world data verify its structure, behavior, and feasibility of the purposes it was built for. Based on these results, it is possible to model a complex phenomenon such as organizational reliability based on published research, especially when the study is designed and implemented rigorously. The combined utilization of thematic analysis, thematic networking, and thematic proximity methods resulted in the development of an FCM model with self-quantified edges as a unique and novel approach to access knowledge purely from literature. When standardized, this approach can contribute to broader research as a new methodology for building and utilizing FCMs and other modeling techniques.

This study proves the importance of selecting the inference function and Lambda, which involves trade-offs for both the sigmoid and the hyperbolic tangent functions. If the results were mapped into the more easily interpreted range of $[0,1]$ or $[-1,1]$, there would be a steep rise in the functions' slope resulting in an increase in the tipping point, which may be difficult for industry experts to understand. Based on a simple comparison test I made as 
shown in Figure 56, I selected Hyperbolic Tangent as an inference function (eq. No. 8) for simulating the model in the expanded with Lambda value of 2 for the reasons discussed above.

An important lesson learned from this pilot study is that providing the participants with suggested causal relationships between the examined interventions and HRO-relevant concepts is not a practical approach, particularly when the nature of the suggested relationships (i.e., increasing or decreasing) is recommended. This approach seemed to have pushed the participants in directions that they might not want to go, based on their experience to the contrary and/or how their organizations are operating. Accordingly, the suggested relationships, their natures, and strengths would be provided to the participants of the expanded study (next chapter) as lists of options to freely choose from based on their experiences and what best fits the organizational and operational settings of their organizations. 


\section{Model Application (Expanded Study)}

In this chapter, I implemented the fourth phase of this dissertation. I performed an expanded study to apply and contextualize the developed model based on larger-scale expert-provided data. Figure 31 illustrates this study's design, which is an iterative process that consisted of several steps. In the first step, I subjected the core model to additional verification tests to further examine it for unforeseen and potential discrepancies. In the second step, I used the same contextualization procedure that I developed and used during the pilot study workshop (see Section 5.2 and Figure 30). However, in this study, I collected the data through an online survey to receive a larger number of responses, including from experts that were not available for an in-person workshop. The survey asked experts to determine the causal connections between the interventions (see Table 14) and HROrelevant concepts in the core model. As I had expected, there were disagreements among the participants about some causal relationships and weights. I handled the resulting uncertainties using EMA. The following sections discuss the steps for conducting this study and the interpretations and analysis of the results.

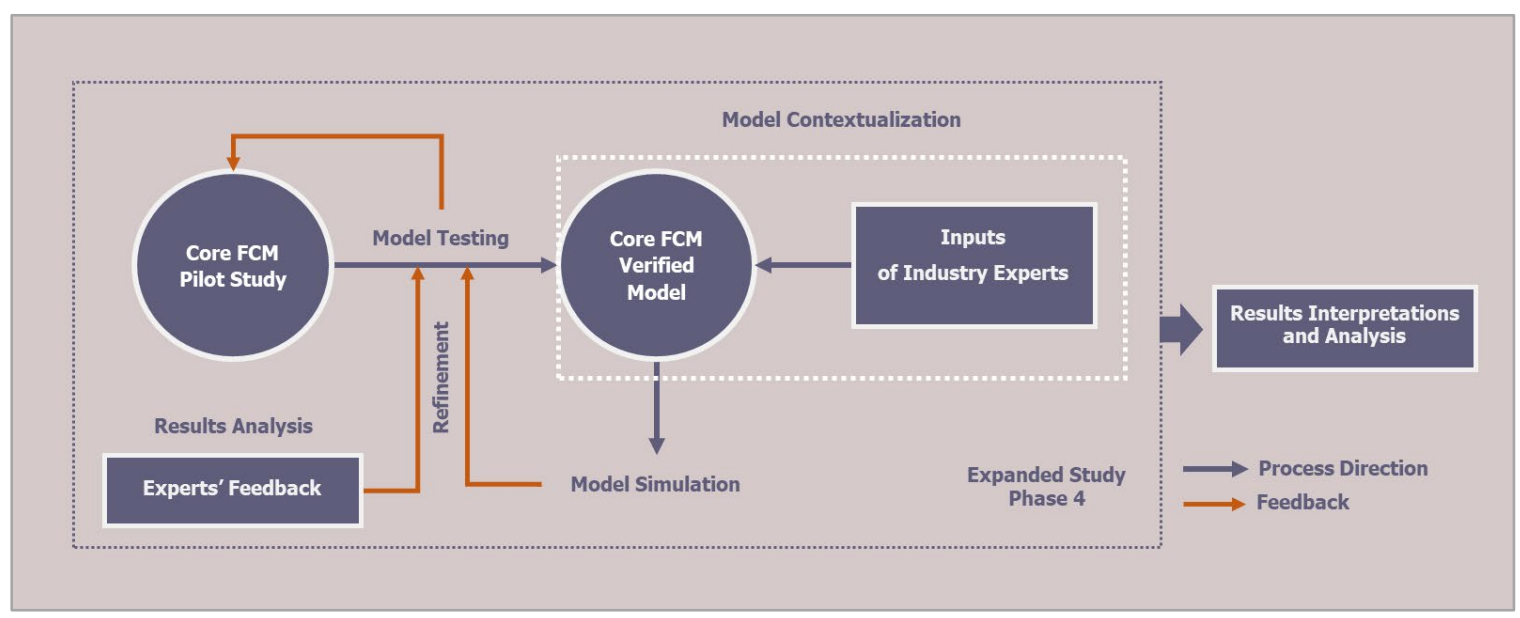

Figure 31: Expanded Study Design 


\subsection{Model Testing for Verification}

I further examined the model's structure and behaviors for any latent issues that the previous tests which I carried out before conducting the pilot study might not capture. Therefore, this series of model testing (discussed in Section 3.7) is another layer of examining the model and prepare it for this expanded study. In these tests, I used qualitative knowledge as a foundation for comparison and verification. The tests I carried out in this study include the model boundary adequacy test as another examination of the model's structure-oriented behavior, behavior anomaly test, family member test, and surprise behavior test. These tests verify the model structure and behaviors and prepare it for research contexts beyond the offshore oil and gas in future research. The following sections discuss how I carried out each one of these tests.

\subsubsection{Boundary Adequacy Test}

To evaluate the relevance of the core model boundaries, I developed the model boundary chart (Sterman, 2000), as shown in Table 16. This chart is developed based on the literature that I used to create the core model, and through which I distinguished between the endogenous $^{20}$, the exogenous ${ }^{21}$, and the excluded ${ }^{22}$ concepts. As mentioned before, the developed core model consists of the concepts that contribute to the five HROT principles and forms the main structure of the complete model of HROT, which includes an outer

\footnotetext{
20 Endogenous elements are the model-driven concepts through which the exogenous elements provide enforced changes. These elements include the concepts of interest (i.e., the five HROT principles).

${ }^{21}$ Exogenous elements are the model driver concepts that form the outer layer of the model. The endogenous elements receive enforced changes through, for example, activating any of the driver concepts or enforcing external changes such as implementing interventions, policies, etc.

22 Excluded elements are concepts that can affect organizational reliability but are not contributing to any of the five principles.
} 
layer that represents safety interventions and how they impact the concepts on the core model's boundary. For example, the concept "Loss of Situational Awareness" is on the core model's boundary; thus, it is exogenous (see Table 16) because it does not change through anything in the core model but, instead, triggers a change in the core model if it is externally increased or decreased. From the perspective of the complete model of HROT, that also includes the outer layer of interventions; it is, however, endogenous.

With respect to the core model, endogenous concepts such as "Failure Knowledge and Attentiveness" (see Table 16) are driven concepts that do not change through anything in the core model unless they are increased or decreased by the core model's exogenous concepts. Because this test is meant to examine the boundary of the core model thus, only endogenous and exogenous concepts listed in Table 16 were examined. By following Approach A of Method A (see Table 7), I simulated the model by activating each exogenous concept individually to assess the effects on the endogenous concepts. This process has been repeated 22 times (the number of exogenous elements) and resulted in verified model behaviors that confirm none of the essential feedback was omitted, which means none of the exogenous concepts have been treated as endogenous and vice versa. Table 16: Core Model Boundary Chart

\begin{tabular}{|l|l|l|}
\hline Endogenous Concepts & Exogenous Concepts & Excluded Concepts \\
\hline Logical Reasoning & Compressed Experience & Managerial Interventions \\
\hline $\begin{array}{l}\text { Anticipating and Controlling } \\
\text { Latent Singles }\end{array}$ & Ignoring True Expertise & $\begin{array}{l}\text { Government Policies, Rules, and } \\
\text { Regulations }\end{array}$ \\
\hline $\begin{array}{l}\text { Failure Knowledge and } \\
\text { Attentiveness }\end{array}$ & Self-Awareness & Industry Regulations \\
\hline Normalization & $\begin{array}{l}\text { Understanding the Situation in } \\
\text { Real-Time }\end{array}$ & Organization Size \\
\hline
\end{tabular}




\begin{tabular}{|c|c|c|}
\hline Precaution as a Mind-Set & Requisite Variety & Production/Service Capacity \\
\hline $\begin{array}{l}\text { Listening to Various Voices and } \\
\text { Inputs }\end{array}$ & Structure Flexibility & Product/Service Demand \\
\hline Misestimating Reliability & Loss of Situational Awareness & Product/Service Price \\
\hline Distinctions & Simple Structure & Labor Market \\
\hline $\begin{array}{l}\text { Obscuring and Neglecting } \\
\text { Significant Information }\end{array}$ & Self-Interest & New Technologies \\
\hline $\begin{array}{l}\text { Organizing for Sense- } \\
\text { Discrediting }\end{array}$ & Loss of Task Attention & $\begin{array}{l}\text { Environment/ Operations Site } \\
\text { Conditions }\end{array}$ \\
\hline Organizational Variety & $\begin{array}{l}\text { Adherence to Standards and } \\
\text { Risk Boundaries }\end{array}$ & \\
\hline $\begin{array}{l}\text { Action Interpretation and } \\
\text { Recursive Interaction }\end{array}$ & Complacency & \\
\hline Elasticity and Recovery & $\begin{array}{l}\text { Organizing for Action-Based } \\
\text { Inquiry }\end{array}$ & \\
\hline Improvisation & Redundancy & \\
\hline Sensemaking & $\begin{array}{l}\text { System Flexibility and } \\
\text { Adaptability }\end{array}$ & \\
\hline Responses (Actions) Repertoire & $\begin{array}{l}\text { Personal Competence and } \\
\text { Confidence }\end{array}$ & \\
\hline Respectful Yielding & Learning from Failure/Incidents & \\
\hline Available Expertise & Domain-Specific Experience & \\
\hline $\begin{array}{l}\text { Sustaining Dynamic Situational } \\
\text { Awareness }\end{array}$ & Overreliance on Experts & \\
\hline Operations as Integrated Map & Pressure & \\
\hline Heedful Interrelating & Level of Employee Participation & \\
\hline Interruptions and Adjustments & Dysfunctional Momentum & \\
\hline \multicolumn{3}{|l|}{ Organizational Reliability } \\
\hline \multicolumn{3}{|l|}{ Preoccupation with Failure } \\
\hline \multicolumn{3}{|l|}{ Reluctance to Simplify } \\
\hline \multicolumn{3}{|l|}{ Sensitivity to Operations } \\
\hline \multicolumn{3}{|l|}{ Commitment to Resilience } \\
\hline Difference to Expertise & & \\
\hline
\end{tabular}




\subsubsection{Behavior Anomaly Test}

This test examines the model for unusual behaviors that result from changing or removing specific causal relationships in the model, or in other words, changing the model structure. Showing any anomalous behaviors when a causal path is changed or deleted infers that this causal path is a part of the model structure. When omitted, the model would not imitate the anticipated behaviors of the phenomena it represents. I conducted this test through the following two steps.

1. I randomly selected three causal paths at the heart of the core model as listed in Table 17, which consists of the model driver concepts in column (A) that indirectly derive changes in the concepts of interest (i.e., the five HROT principles) in column (C), through the intermediate model concepts listed in column (B).

2. I simulated the model following Approach $\boldsymbol{A}$ - Method $\boldsymbol{A}$ (see Table 7) for each case individually before and after deleting the mediating concepts in column (B).

The results of simulating the model for each case are graphically shown in Figure 32, which compares the five HROT principles' behaviors in each case before and after deleting the intermediate concepts in column (B).

Depending on how each intermediate concept is defined, and since these intermediate concepts have direct relationships with the five HROT principles, their influences on the principles' behaviors were predicated. For example, in the first case, deleting "Complacency" has increased the degree of change in each of the four affected principles, and notably, that PWF resulted in an increasing outcome. This is, in fact, an abnormal (anomalous) behavior because complacency is a cultural trait that exists in every 
organization, and its adverse effects cannot be removed simply by eliminating it rather than coping with it during the ordinary course of operations. Thus, complacency is an essential concept in the model to mediate the relationship between redundancy and the five principles to consistently warn the decision-makers that redundancy can result in adverse effects.

Table 17: Selected Cases for Behavior Anomaly Test

\begin{tabular}{|c|c|c|c|c|c|}
\hline Case & $\begin{array}{c}\text { (A) } \\
\text { Driver Concept }\end{array}$ & & $\begin{array}{c}\text { (B) } \\
\text { Mediated Concept } \\
\text { (To Be Removed) }\end{array}$ & & $\begin{array}{c}\text { (C) } \\
\text { Concepts of Interest }\end{array}$ \\
\hline 1 & Redundancy & $-\rightarrow$ & Complacency & $-\rightarrow$ & Five Principles \\
\hline 2 & Precaution as a Mind-set & $+\rightarrow$ & Sensemaking & $+\rightarrow$ & Five Principles \\
\hline 3 & $\begin{array}{l}\text { Interruption and } \\
\text { Adjustment }\end{array}$ & $+\rightarrow$ & $\begin{array}{c}\text { Anticipating and } \\
\text { Controlling Latent Singles }\end{array}$ & $+\rightarrow$ & Five Principles \\
\hline
\end{tabular}




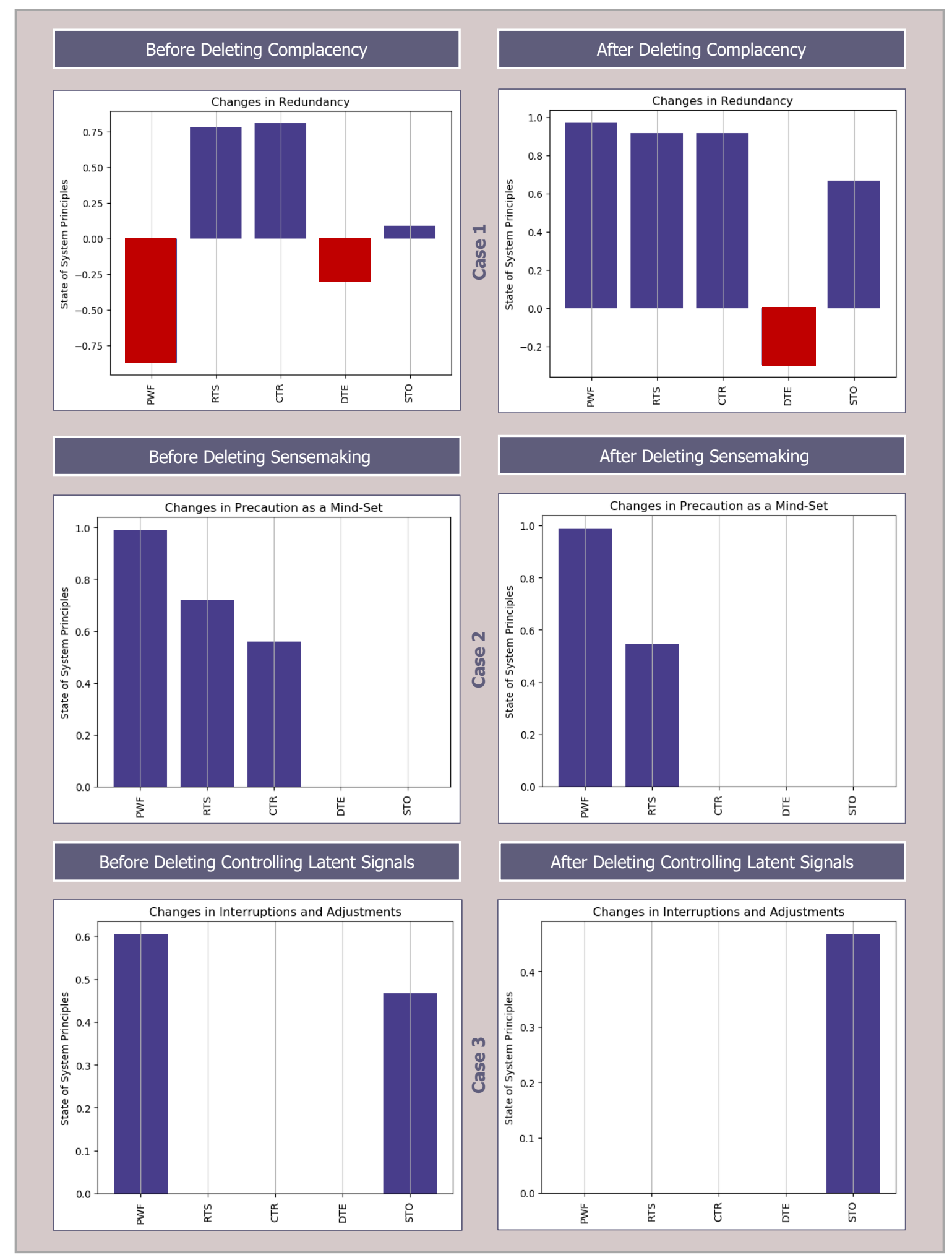

Figure 32: Changes in the Five HROT Principles Based on Behavior Anomaly Test 


\subsubsection{Family Member Test}

This test required simulating the model based on at least two different contexts (industries or organizations). Each simulation is expected to show behaviors that imitate the behaviors of the context it represents, as described in the literature. I selected the healthcare industry besides the offshore oil and gas to make this comparison since there is a rich literature on healthcare concerning practicing HROs since 2001.

Frankel et al. (2006) urge that several US healthcare organizations took initiatives to enhance safety, including the introduction of the so-called "red rules," which, if not followed, can result in potential dismissal (Jones \& O'Connor, 2016). Such rules infer that a culture of raising concerns regarding patients' care is not fostered or is deficient. However, raising concerns in offshore oil and gas claimed to be encouraged and rewarded in some organizations (Lekka \& Sugden, 2011; National Academies, 2016). This specific behavioral aspect (i.e., raising concern) is a part of the model structure that defines and evaluates the HRO-relevant concept "Listening to Various Voices and Inputs."

I, therefore, simulated the model following Approach $\boldsymbol{A}-\operatorname{Method} \boldsymbol{A}$ (see Table 7) to measure the degree of change in the five HROT principles based on changing the level of activating this concept, considering a low degree in healthcare while an adequate degree in the offshore oil and gas. Simulating the model based on that has resulted in behaviors that are verifying what has been described in the literature regarding the two selected industries, which infer that the model has passed the test as shown in Figure 33. It is evident that RTS, STO, and CTR have shown less degrees in healthcare since people in this industry are less 
allowed to improvise when needed, less to communicate issues, and they are mostly regulated to treat cases based on similar contexts.

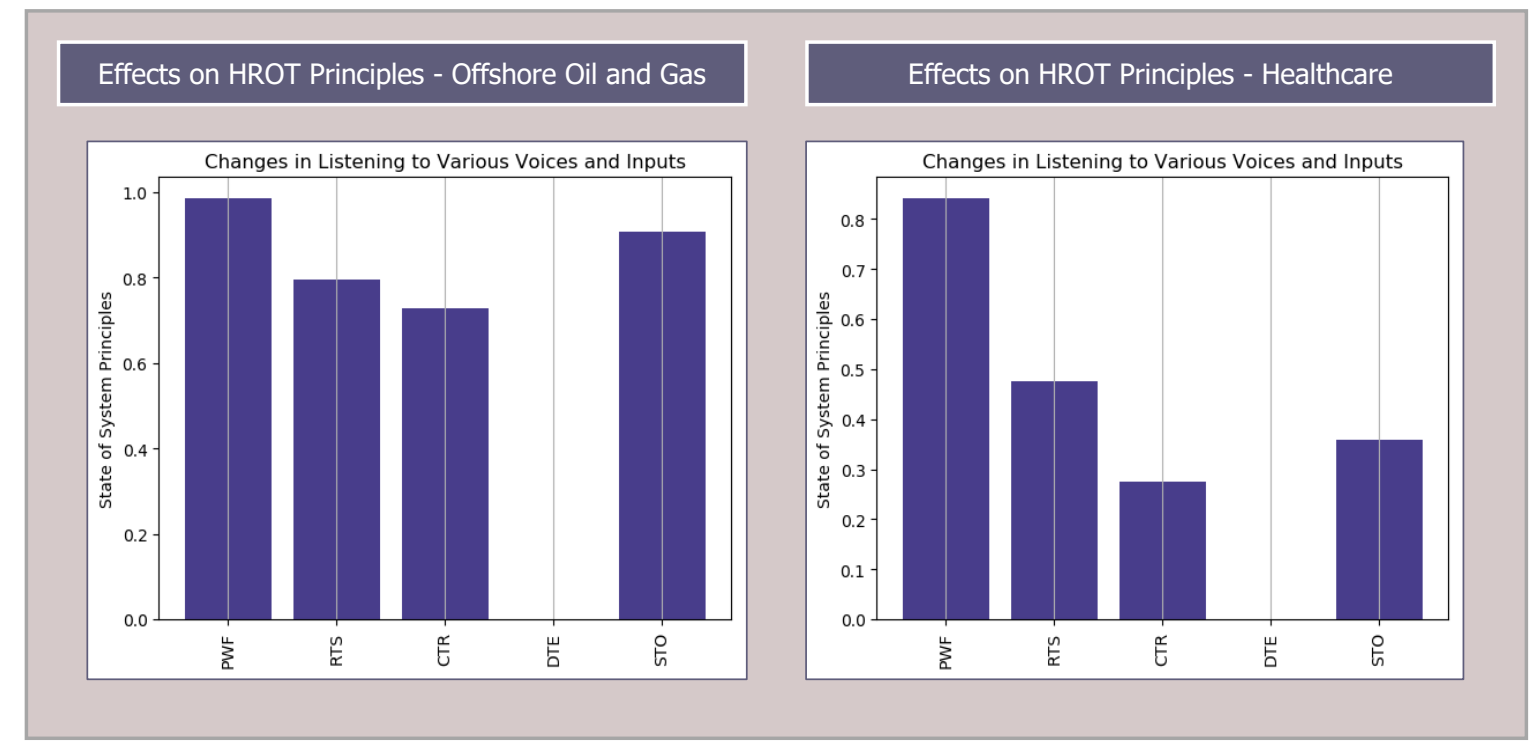

Figure 33: Simulation Results of the Family Member Test

\subsubsection{Surprise Behavior Test}

Testing the model based on a surprise behavior test required subjecting it to an external force (i.e., managerial intervention) through a list of HRO-relevant concepts. I simulated the model following Approach A - Method B (see Table 7) and graphically illustrated in Figure 30. This test required experts' opinions to establish and weigh the relationship between the applied intervention and model concepts so that the model can mimic the realworld system's behaviors. Therefore, I used the experts' feedback provided at the end of the pilot study workshop.

However, in real-world operations, practical implementation of some managerial interventions can generate conflicts with other interventions, programs, and initiatives that organizations have already applied. Consequently, in real life, a theoretically increasing 
relationship between the HRO-relevant concept and the examined intervention is decreasing, so managers can manage any possible conflicts. Indeed, I observed this case when I linked the model to one of the interventions, namely "Fostering Social Ties Based on Mutual Respect" (see Table 14 for definition). One of the HRO-relevant concepts that this intervention assumed to increase is "Organizing for Action Based Inquiry" (See Table 30 for definition). However, experts perceived this relationship decreasing during the workshop since this intervention was found to create conflict with another intervention.

Simulating the model based on the experts' opinions has generated a specific behavior that can exist in the real-world operation but is not theoretically anticipated, which indicated that the model had passed the test. Figure 34 compares the model behavior based on the literature (left) and based on the industry experts' opinions (right) regarding this case.

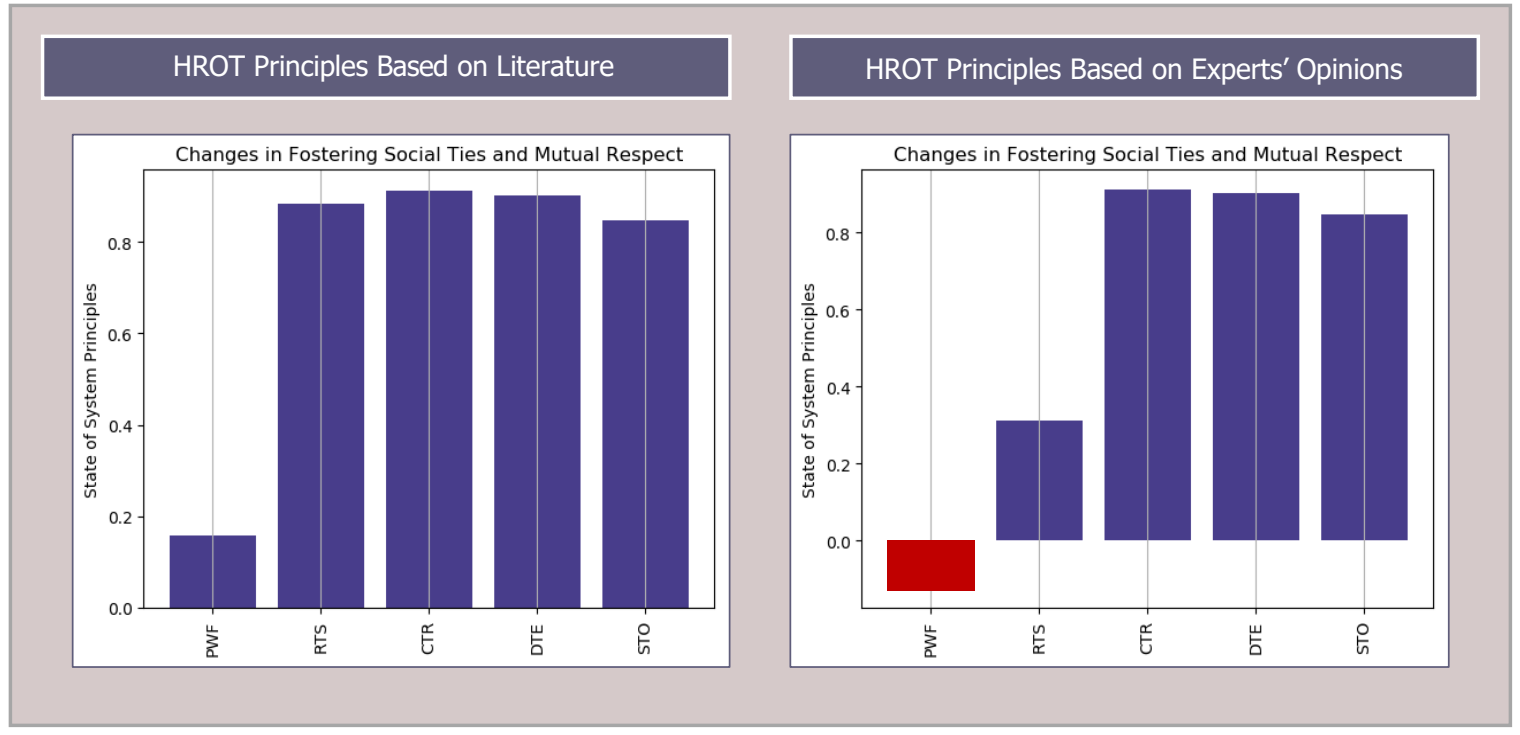

Figure 34: Model Behavior Based on Literature VS. Experts' Opinions 


\subsection{Data Collection}

I collected the required data for this expanded study through an online survey that I designed based on the model contextualization procedure to gather information from the offshore oil and gas industry experts. The following sections discuss the participants and the recruitment process, and the survey design and administration.

\subsubsection{Participants and Recruitment}

Like the pilot study, industry participants in this expanded study play an essential role in contextualizing the model based on their organizations' organizational and operational settings. Therefore, the participants' experiences and their role in safety-related practices have been a significant concern, making the process of recruiting them critical and challenging. Practitioners from the offshore oil and gas are difficult to reach out to, and when possible, they mostly apologize to take part due to the sensitivity of their duties and the fear that such studies may risk their jobs. However, there have been three channels to recruit participants: (1) Business cards and personal information that I collected from the attendees of the offshore oil and gas 2017 and 2018 annual forum by Center for Offshore Safety COS in Houston, Texas, (2) a list of email addresses provided by the COS, and (3) LinkedIn accounts as I searched participants with different expertise and safety positions in the offshore oil and gas.

Participants' roles that I recruited to participate in the survey included: HSE or HSSE managers, IDAC members and consultants, Offshore rig administrators, safety managers, safety auditors, safety directors, safety officers, safety specialists, safety coordinators, safety advisors, safety influencers, safety trainers, safety consultants, compliance 
managers, compliance coordinators, industrial psychologists, risk managers, operations controllers, operators. The final list consisted of 150 experts with different positions and responsibilities who presently or previously worked in leading and pioneering offshore oil and gas organizations such as Chevron, ExxonMobil, PB, Schlumberger, Halliburton, Baker Hughes, Rowan Companies, Subsea7, and others.

\subsubsection{Survey Design and Administration}

I designed and created an online survey with Qualtrics to collect the required data for this study. The survey is designed to provide a one by one defined intervention and a one by one question regarding whether increasing the intervention's implementation would increase, decrease, or make no change on a defined HRO-relevant concept. This survey examined thirteen interventions (see Table 14), and each intervention has been questioned several times based on the number of potential HRO-relevant concepts (see Table 35 in Appendix G). Participants responded to the questions by selecting one of the given options to indicate a relationship, the relationship's nature (i.e., increasing or decreasing), and its strengths, as shown in Figure 35.

This design aims to examine the positive and negative effects of the thirteen selected managerial interventions on the five HROT principles. This process facilitated studying the cultural behaviors and the structural design and patterns of the research context in realworld operations. It revealed the strengths and weaknesses of safety-related practices, reflecting the industry's capabilities of either becoming or continuously practicing HROs. Because surveying participants regarding thirteen interventions is a time-consuming 
process and somehow overwhelming, therefore, the survey is designed to automatically randomize and limit surveying each participant about only two interventions.

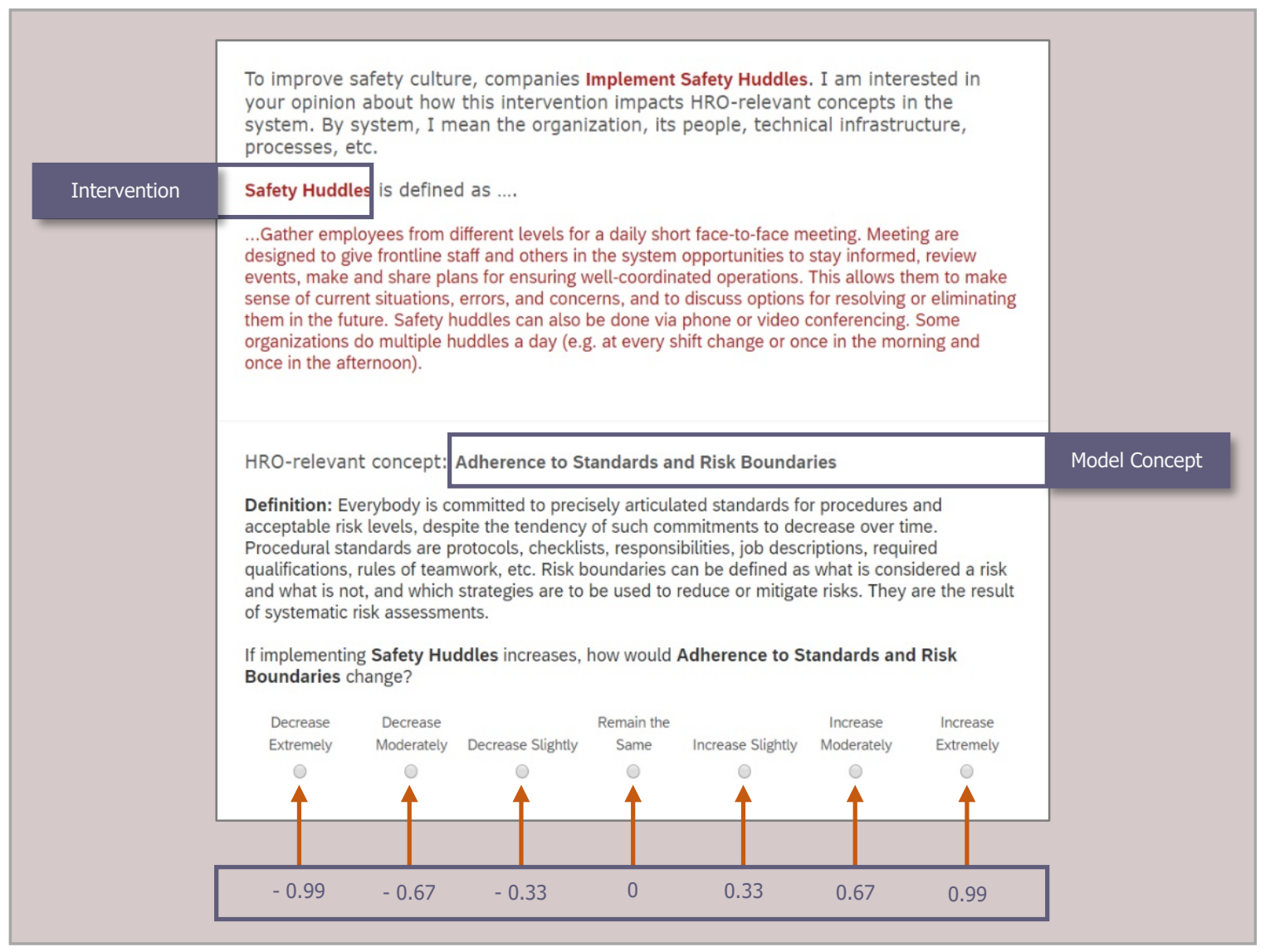

Figure 35: Screen Shot of the Expanded Study Online Survey with Qualtrics

I tested this survey for its flow, clarity, and effectiveness using the Qualtrics software's testing data. Then I distributed it through multiple channels to reach out to the previously identified participants from the offshore oil and gas industry.

The survey was distributed to 150 potential experts via their email addresses and directly through their LinkedIn accounts; however, only 31 complete responses and 17 partial responses with a percentage of completion between $50-85 \%$ have been recorded. I considered responses with $70 \%$ partial completion and above in the final use of the data; 
therefore, the data points' final sample consisted of 40 usable responses. Furthermore, I analyzed the data using Qualtrics for collecting the responses per each causal relationship between the examined interventions and every HRO-relevant concept-the analyzed data presented in the following sections of the model simulation and behaviors.

\subsection{Model Simulation and Behaviors}

In this section, the discussions comprise simulating the model based on the selected managerial interventions. The simulation process included using two techniques: Consolidative FCM as a traditional simulation method, and explorative FCM as an Exploratory Modelling and Analysis (EMA) method. The purpose of this lengthy process is threefold:

1. Subjecting the model to more managerial interventions and a more comprehensive range of expert-provided data to examine in depth its appropriateness and usefulness.

2. Evaluating the capacity and readiness of the offshore oil and gas operations for practicing HROs based on experts' opinions.

3. Managing uncertainties resulted from experts' disagreements around specific causal relationships, thus, understanding the effects of different management styles and decisions on safety-related practices and behaviors.

This section presents the process and results of simulating the model based on the collected data; however, the detailed analysis and interpretations of these results are provided in section 6.4 . 


\subsubsection{Traditional FCM Simulation}

In the traditional model simulation method, I applied the selected managerial interventions to the core model through lists of the HRO-relevant concepts based on the previously discussed contextualization procedure (see Table 35 in Appendix G). The nature and weight of each intervention's causal relationship with the corresponding HRO-relevant concepts have been calculated using the median of the collected data. The median provides the middle value or the central tendency of the data set so that disagreements among participants concerning a specific causal relationship can be resolved. For instance, if three participants disagreed around a specific relationship: the first selected slightly increasing $(+0.33)$, the second chose moderately increasing $(+0.67)$, and the third selected moderately decreasing $(-0.33)$, the final determination based on the calculated median is slightly increasing relationship (+0.33). Table 39 in Appendix I) consists of the resulted edges' weight of all the relationships based on the median of the experts' opinions.

I simulated the model using Approach $\boldsymbol{A}$ - Method B (see Table 7). I used hyperbolic tangent (tanh (x), Eq. No. 8) as an inference function, and I selected lambda's value to be 2. The selected thirteen interventions have been activated individually and then collectively (i.e., as groups) by clustering them based on each intervention's expected outcomes. The following sections discuss the simulation process based on individual (one by one intervention) and then groups of interventions.

\subsubsection{Simulation-Based on Individual Interventions}

I simulated the model based on individual interventions by fully activating ( $100 \%$ turning out) one intervention at a time and let the system reaches the study state. I visualized each 
intervention's effects on the five HROT principles (concepts of interest) using bar graphs. The degree of change in each principle presented graphically, as shown in Figure 36 and Figure 37. Simultaneously, the numerical outcomes based on these simulation processes are provided in Table 18, including the five HROT principles and Organizational Reliability (OR).

Importantly, I calculated the degree of OR using the five principles' sum-product, considering that each principle is equally participating in making the degree of organizational reliability OR (i.e., each principle represents $20 \%$ of the OR's degree). For examples, if: $\mathrm{PWF}=0.75$,

$\mathrm{RTS}=0.68$,

$\mathrm{STO}=0.00$,

$\mathrm{CTR}=0.85$,

$\mathrm{DTE}=0.25$, therefore:

$\mathrm{OR}=(0.75 * 20 \%+0.68 * 20 \%+0.00 * 20 \%+0.85 * 20 \%+0.25 * 20 \%)=0.51$.

Figure 36 and Figure 37, as well as Table 18, show that only seven interventions out of the thirteen selected and applied to the model have positively influenced all the five HROT principles simultaneously as they presented increasing degrees of change. These interventions include Post-event debriefings, tabletop exercises, emergency drills, personal accountability, social ties and mutual respect, prosocial motivation, and emotional ambivalence. These managerial interventions should be given further attention by the industry to develop positive and higher organizational reliability levels. Furthermore, communication as a means to stay informed by huddling and reporting issues has positive 
impacts on only four principles, which is expected since communication does not directly affect the fifth principle (i.e., DTE) similar to what, for example, training interventions have. However, communication in real-time as a method for learning has yielded counterproductive effects. In contrast, the stop-work authority has shown adverse effects, particularly on PWF, considering that interventions resulting in decreasing or negative degrees of change are undesired and wasteful management efforts.

Notably, there have been several cases where the model simulation based on individual interventions has shown that maximizing the activation level or implementing an intervention does not always result in desired outcomes. For example, the full activation level (100\% turning on) of "Fostering Social Ties and Mutual Respect" has positively influenced PWF with a change of (0.453) while activating the same intervention with a half activation level (50\% turning on) has resulted in a higher degree of PWF (0.575) as shown in Figure 38 below. More details on results interpretation and analysis are provided in Section 6.4. 


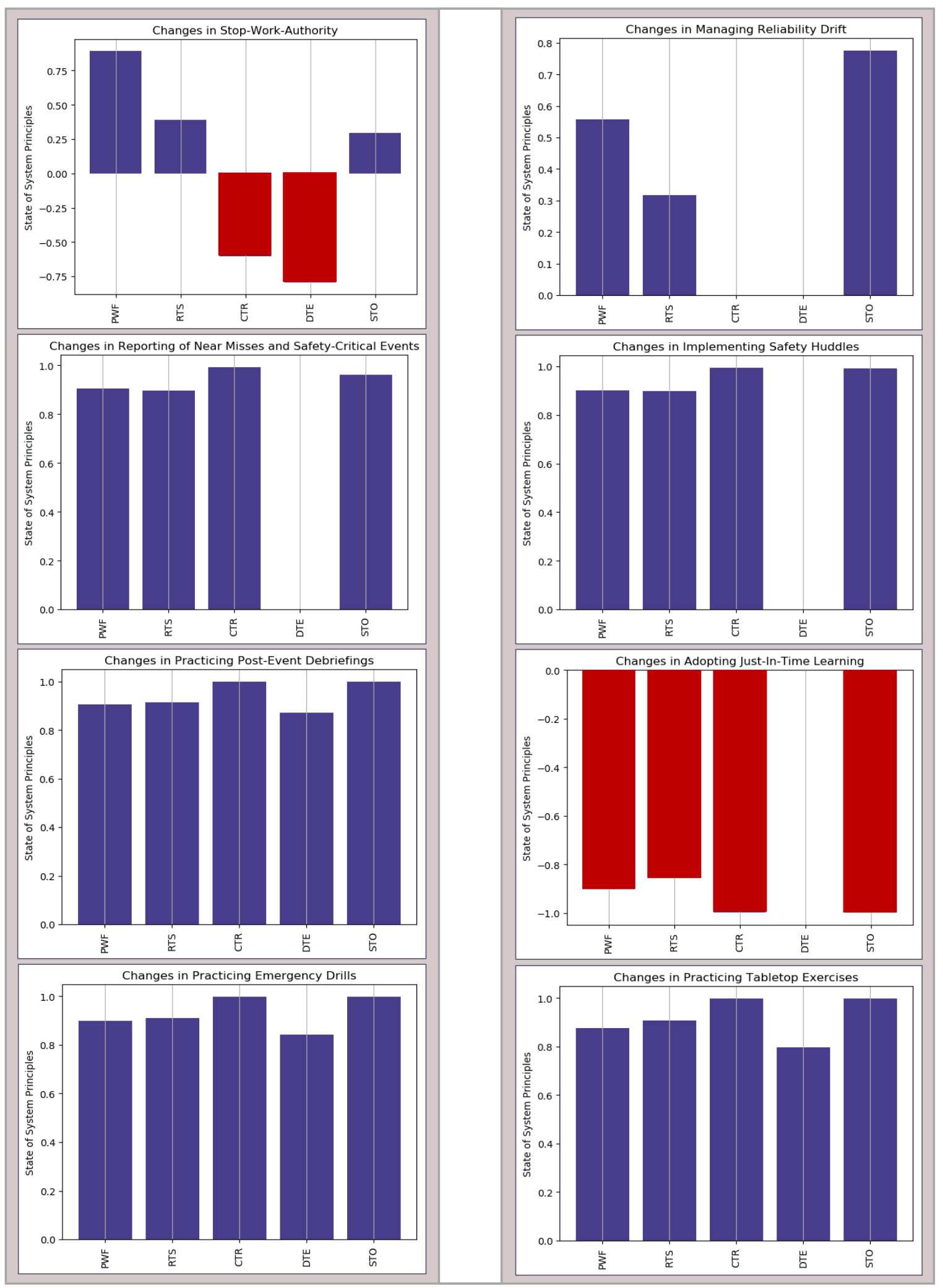

Figure 36: Model Simulation Based on Individual Interventions (1) 


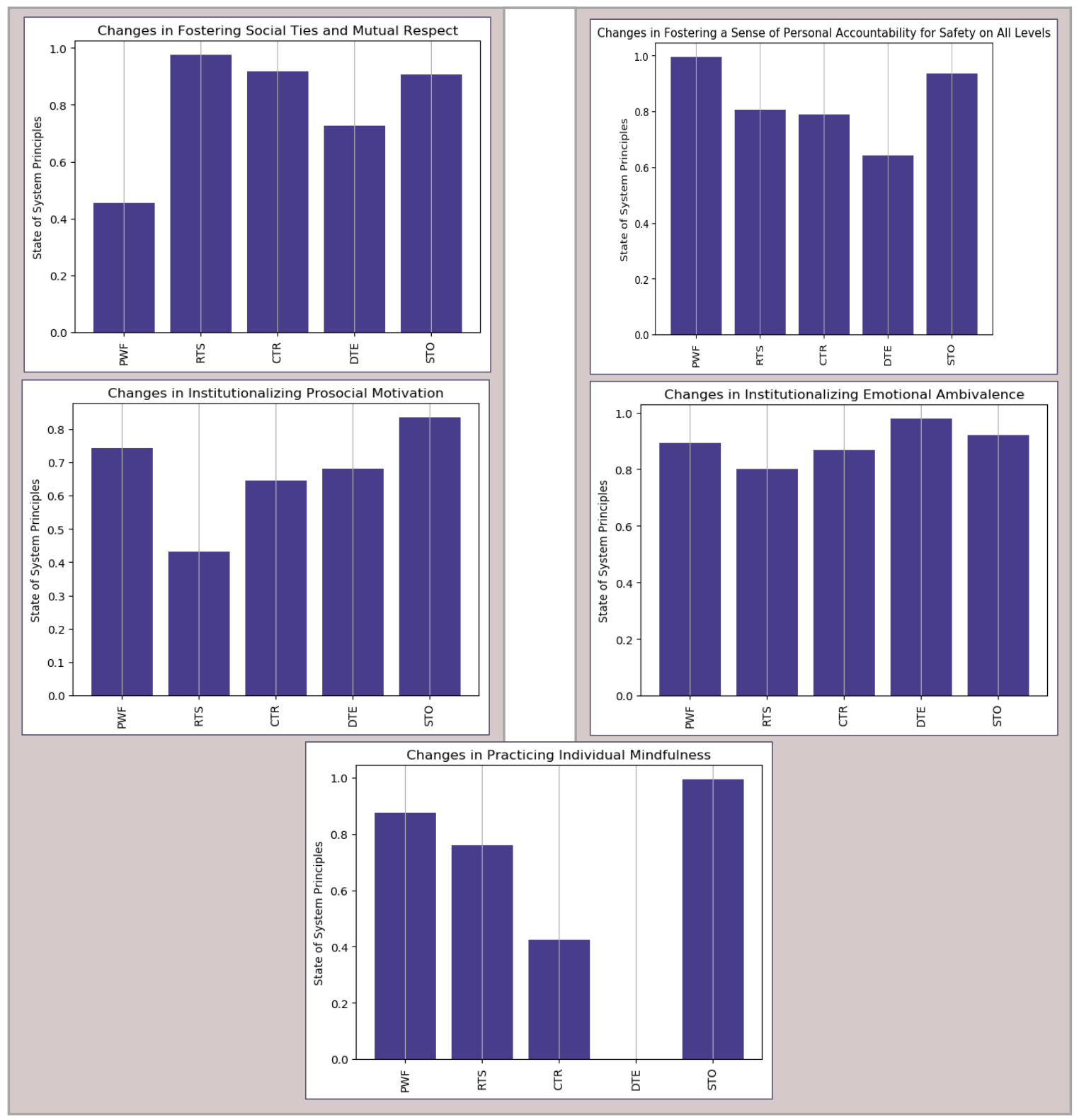

Figure 37: Model Simulation Based on Individual Interventions (2) 
Table 18: Numerical Outcomes of the Five HROT Principles and Organizational Reliability (Based Individual Interventions)

\begin{tabular}{|c|c|c|c|c|c|c|}
\hline Interventions & $P W F$ & RTS & CTR & DTE & STO & OR \\
\hline Stop-Work Authority & 0.891 & 0.391 & -0.602 & -0.796 & 0.296 & 0.036 \\
\hline Reporting of Near Misses and Safety-Critical Events & 0.906 & 0.896 & 0.993 & 0.000 & 0.962 & 0.751 \\
\hline Fostering Social Ties and Mutual Respect & 0.453 & 0.977 & 0.917 & 0.728 & 0.908 & 0.797 \\
\hline Fostering a Sense of Personal Accountability for Safety on All Levels & 0.995 & 0.805 & 0.780 & 0.641 & 0.936 & 0.831 \\
\hline Implementing Safety Huddles & 0.901 & 0.897 & 0.994 & 0.000 & 0.992 & 0.757 \\
\hline Practicing Tabletop Exercises & 0.876 & 0.906 & 0.997 & 0.796 & 0.998 & 0.915 \\
\hline Practicing Post-Event Debriefings & 0.906 & 0.914 & 0.998 & 0.872 & 0.999 & 0.938 \\
\hline Practicing Emergency Drills & 0.901 & 0.910 & 0.998 & 0.842 & 0.998 & 0.930 \\
\hline Institutionalizing Prosocial Motivation & 0.742 & 0.432 & 0.644 & 0.680 & 0.835 & 0.667 \\
\hline Institutionalizing Emotional Ambivalence & 0.893 & 0.802 & 0.867 & 0.979 & 0.922 & 0.893 \\
\hline Managing Reliability Drift & 0.557 & 0.317 & 0.000 & 0.000 & 0.775 & 0.330 \\
\hline Practicing Individual Mindfulness & 0.875 & 0.759 & 0.424 & 0.000 & 0.994 & 0.610 \\
\hline Adopting Just-In-Time Learning & -0.900 & -0.854 & -0.995 & 0.000 & -0.998 & -0.750 \\
\hline
\end{tabular}




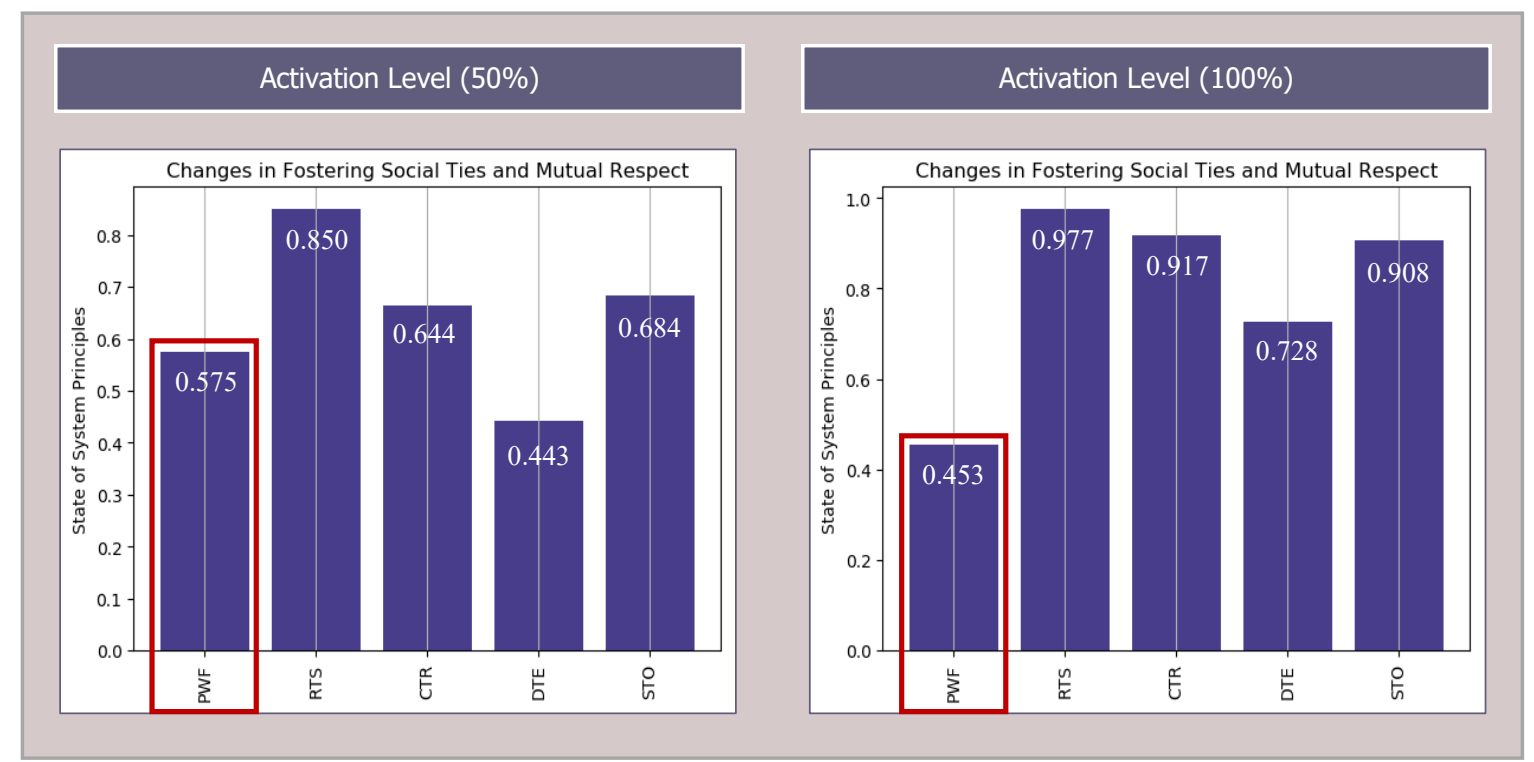

Figure 38: Comparison of Model Simulation Based on Different Activation Levels

\subsubsection{Scenarios Based on Grouped Interventions}

In their efforts to improve safety-related practices, managers do not just do one thing. Still, in selecting interventions, they tend to focus on areas such as workforce training, work design, communications, and so forth. Therefore, I developed scenarios based on grouping the selected managerial interventions into five groups considering the anticipated improvements that these interventions are designed to achieve, as listed in Table 19. Simulating the model based on these scenarios required activating each group and letting the system reach the study state. The effects of each group on the five HROT principles are visualized using a bar graph, by which the degree of change in each principle is presented, as shown in graphs A, B, C, D, and E in Figure 39. Moreover, the numerical outcomes of this simulation process are provided in Table 20, which includes the degrees of change in the performance of the five HROT principles as well as organizational reliability (OR). 
From the results of simulating the model based on individual interventions (see Figure 36 and Figure 37), some interventions have positively influenced the five HROT better than others within the same cluster or group of interventions (see Table 18 and Table 19). I grouped these interventions with the best influences under a category of best performance interventions. Then I followed all the required steps to simulate the model as a consolidative FCM based on this group. The outcomes of this process are presented in graph (F) in Figure 39, and the numerical values are provided in Table 20.

It is apparent from the simulation results that the group of reframing work training has achieved a very ambitious level of OR, which is clearly demonstrated as very high degrees of change in the performance of all the five HROT principles that range between (0.88) to (0.99). However, like simulating the model based on individual interventions, this experience has shown that not every group of interventions could influence all the five principles concurrently, such as reframing work communication. Grouping interventions do not always result in the same or similar positive degrees of change in all the five principles, such as simulating the model based on reframing work design. Moreover, grouping best performance interventions yielded an ideal level of OR; however, these ideal results pose two questions: (1) how costly or feasible is to design and implement a group of interventions to reach such an outcome, and (2) how possible in practice to implement all these interventions at the same time without developing any counterproductive effects? Such questions worth investigation in future research. More details on the results analysis and interpretations are provided in Section 6.4. 
Table 19: Scenario Based on Grouped Interventions

\begin{tabular}{|c|c|c|}
\hline Scenario/Cluster Name & Interventions & Description \\
\hline Reframing Work Design & $\begin{array}{l}\text { - Stop-Work Authority } \\
\text { - Managing Reliability Drift } \\
\text { - Institutionalizing Emotional } \\
\text { Ambivalence } \square\end{array}$ & $\begin{array}{l}\text { These interventions are meant to reframe how the work is designed to manage } \\
\text { emergencies, resolve unforeseen mishaps, and deviations from procedures. By } \\
\text { doing so, management authorizes front-line workers to stop work when needed. } \\
\text { This design motivates workers to trust their competence yet continue to consult } \\
\text { experts when required. }\end{array}$ \\
\hline $\begin{array}{l}\text { Reframing Work } \\
\text { Communication }\end{array}$ & $\begin{array}{l}\text { - Implementing Safety Huddle } \\
\text { - Reporting Near-Misses/ Incidents } \square\end{array}$ & $\begin{array}{l}\text { These two interventions practiced leveraging the level and quality of } \\
\text { communications, raising concerns, receiving and passing instructions, and } \\
\text { getting informed about mishaps solutions and recommendations on how to avoid } \\
\text { them. }\end{array}$ \\
\hline $\begin{array}{l}\text { Reframing Work } \\
\text { Training }\end{array}$ & $\begin{array}{l}\text { - Emergency Drills } \\
\text { - Tabletop Exercise } \\
\text { - Just-In-Time-Learning } \\
\text { - Post-Events Debriefings } \square\end{array}$ & $\begin{array}{l}\text { These interventions do not provide essential training but rather train workers and } \\
\text { prepare them for pre and post emergencies as well as keep workers informed and } \\
\text { trained in real-time while they perform their jobs. }\end{array}$ \\
\hline $\begin{array}{l}\text { Reframing How to Relate } \\
\text { Colleagues Peers }\end{array}$ & $\begin{array}{l}\text { - Institutionalize Precocial Motivation } \\
\text { - Fostering Social Ties and Mutual } \\
\text { Respect } \square\end{array}$ & $\begin{array}{l}\text { Management intervenes to develop and promote work environments that } \\
\text { encourage social activities that strengthen how colleagues' peers are relating to } \\
\text { each other. These activities also motivate workers to follow the standards and } \\
\text { rules that ensure the safety of the self and others' safety yet maintain a high level } \\
\text { of respect. }\end{array}$ \\
\hline $\begin{array}{l}\text { Reframing How the } \\
\text { Work Should Be Done }\end{array}$ & $\begin{array}{l}\text { - Practicing Individual Mindfulness } \\
\text { - Fostering Sense of Accountability and } \\
\text { Safety on All Levels } \square\end{array}$ & $\begin{array}{l}\text { Management intervenes to affirm that everyone is accountable for his/her job } \\
\text { duties, particularly concerning safety-related practices. The workforce is } \\
\text { practicing individual mindfulness, which is some self-actions to clear out their } \\
\text { thoughts and strengthen their abilities to stay focused on their work } \\
\text { responsibilities. }\end{array}$ \\
\hline $\begin{array}{l}\text { Best Performance } \\
\text { Interventions }\end{array}$ & $\begin{array}{l}\text { - Five Best Performance Interventions } \\
\text { Marked with } \square \text { Sign }\end{array}$ & $\begin{array}{l}\text { The interventions in this scenario represent the interventions that showed the best } \\
\text { outcomes for the five HROT principles. }\end{array}$ \\
\hline
\end{tabular}




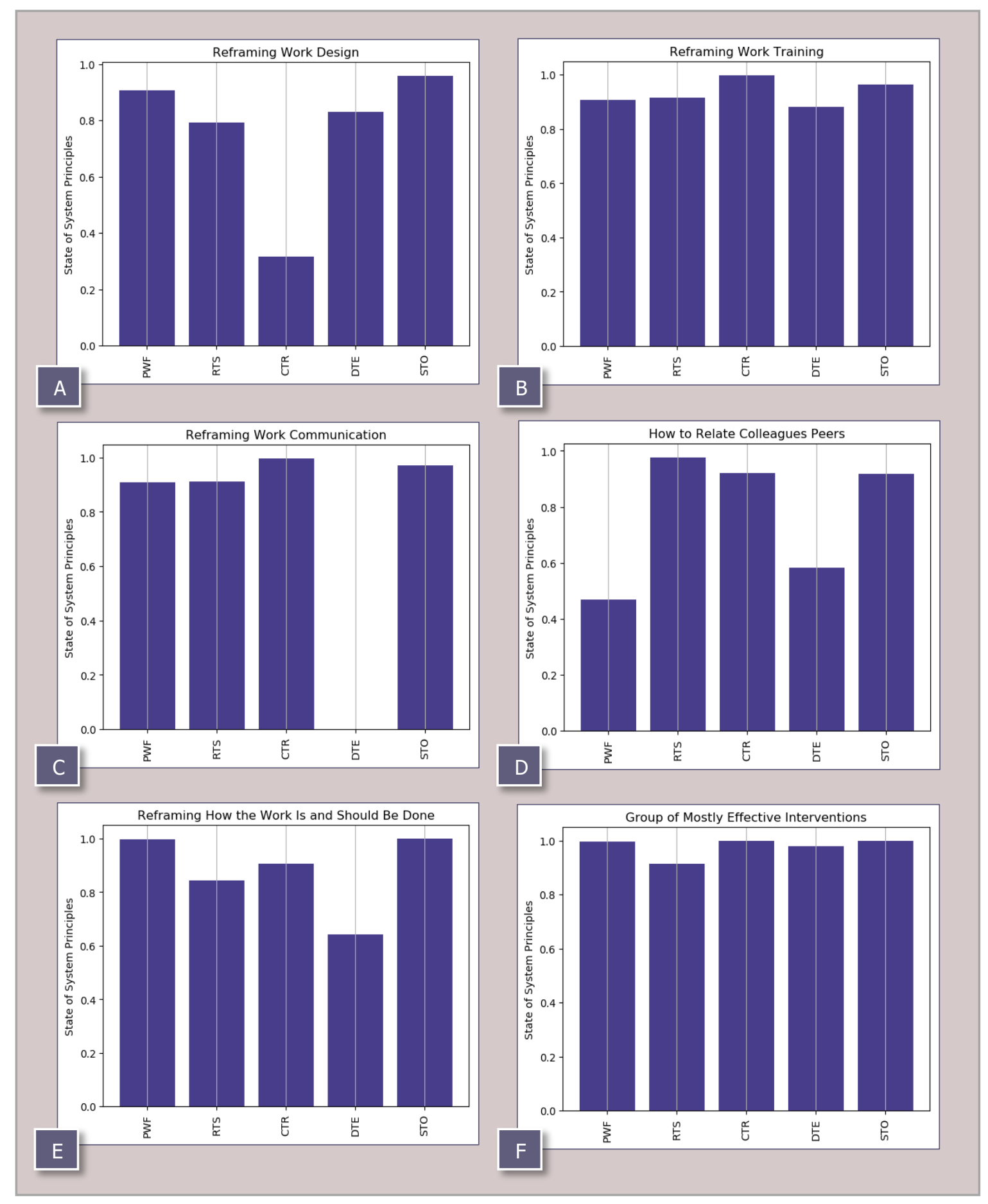

Figure 39: Model Simulation Based on Grouped Interventions 
Table 20: Numerical Outcomes of the Five HROT Principles and Organizational Reliability Based on Grouped Interventions

\begin{tabular}{|c|c|c|c|c|c|c|}
\hline Interventions & $P W F$ & $R T S$ & $C T R$ & $D T E$ & STO & OR \\
\hline Work Design & 0.906 & 0.790 & 0.316 & 0.830 & 0.957 & 0.759 \\
\hline Work Communication & 0.907 & 0.910 & 0.996 & 0.000 & 0.970 & 0.756 \\
\hline Work Training & 0.907 & 0.914 & 0.997 & 0.881 & 0.964 & 0.932 \\
\hline Relating Colleagues Peers & 0.468 & 0.976 & 0.921 & 0.582 & 0.919 & 0.773 \\
\hline How the Work Should Be Done & 0.995 & 0.845 & 0.906 & 0.641 & 0.999 & 0.877 \\
\hline Groupe of Best Performance Interventions & 0.992 & 0.932 & 0.993 & 0.941 & 0.993 & 0.999 \\
\hline
\end{tabular}




\subsubsection{Explorative FCM Simulation Using EMA Technique}

The participants of the industry survey did not agree on the weights for all connections between interventions and the core model concepts, resulting in uncertainty about what model structure best represents expert knowledge. As planned, I conducted EMA to address this uncertainty. However, EMA is computationally demanding and timeconsuming, which is why I first subjected the model to a series of sensitivity analysis tests to identify causal relationships between the interventions and HRO-relevant concepts for which the choice of weights has an impact on HROT principles.

The survey allows participants to (1) decide whether or not there is a relationship between the intervention and each HRO-relevant concept, and (2) choose a sign and a strength at the same time when they decide that there is a relationship, so that, uncertainty or disagreement ranges among participants includes both the strength of the relationship and its sign. For example, if three participants disagree around a relationship: one decided that there is no impact, or no relationship (selected 0 ), the second decided that there is a slightly decreasing impact (selected -0.33 ), and the third decided that there is a moderately increasing impact ( selected +0.66 ); therefore, the uncertainty range is $[-0.33,+0.66]$. The survey results show that 52 out of 70 causal relationships, where participants disagreed on the sign and strength of the relationships (i.e., provided different inputs. The extreme range of uncertainty based on the results is $[-0.66,+1]$, and a typical range is $[0,+0.33]^{23}$. The subsequent EMA discussions focused on the relationships with uncertainty ranges only.

23 I consider this range typical since the uncertainty interval is relatively small, which means that the generated random numbers (selected to be 100,000) would yield similar and more concentrated results. 


\subsubsection{Sensitivity Analysis Test}

As discussed in Sections 3.8.1 and graphically illustrated in Figure 30, the model has been tested for sensitivity following Approach B - Method B (see Table 7) for model simulation. Each intervention was individually activated to examine only one HRO-relevant concept at a time. Individual examination of each concept required ranging the edge's weight from $[-1]$ to $[+1]$ while fixing the other edges' weights to the default values. The default value means [+1] for all the increasing relationship and [-1] for all decreasing ones. In this process, I selected hyperbolic tangent $(\tanh (\mathrm{x}))$ as an inference function, and I set the lambda value to 2 .

I repeated this test 52 times (the number of the critical relationships) to examine each causal relationship with uncertainty range, yet only 20 causal relationships where one or more of the five principles have shown sensitivity to the variations in the edges' weight. These 20 cases are modeled and simulated using the EMA technique, which is discussed in detail in the next section.

Figure 40 below compares some examples of sensitive and insensitive behaviors of the five HROT principles resulted from this step, and Table 21 lists the 20 cases where the HROT principles were found to be sensitive, including the corresponding ranges of uncertainties based on the collected data via the online survey. 

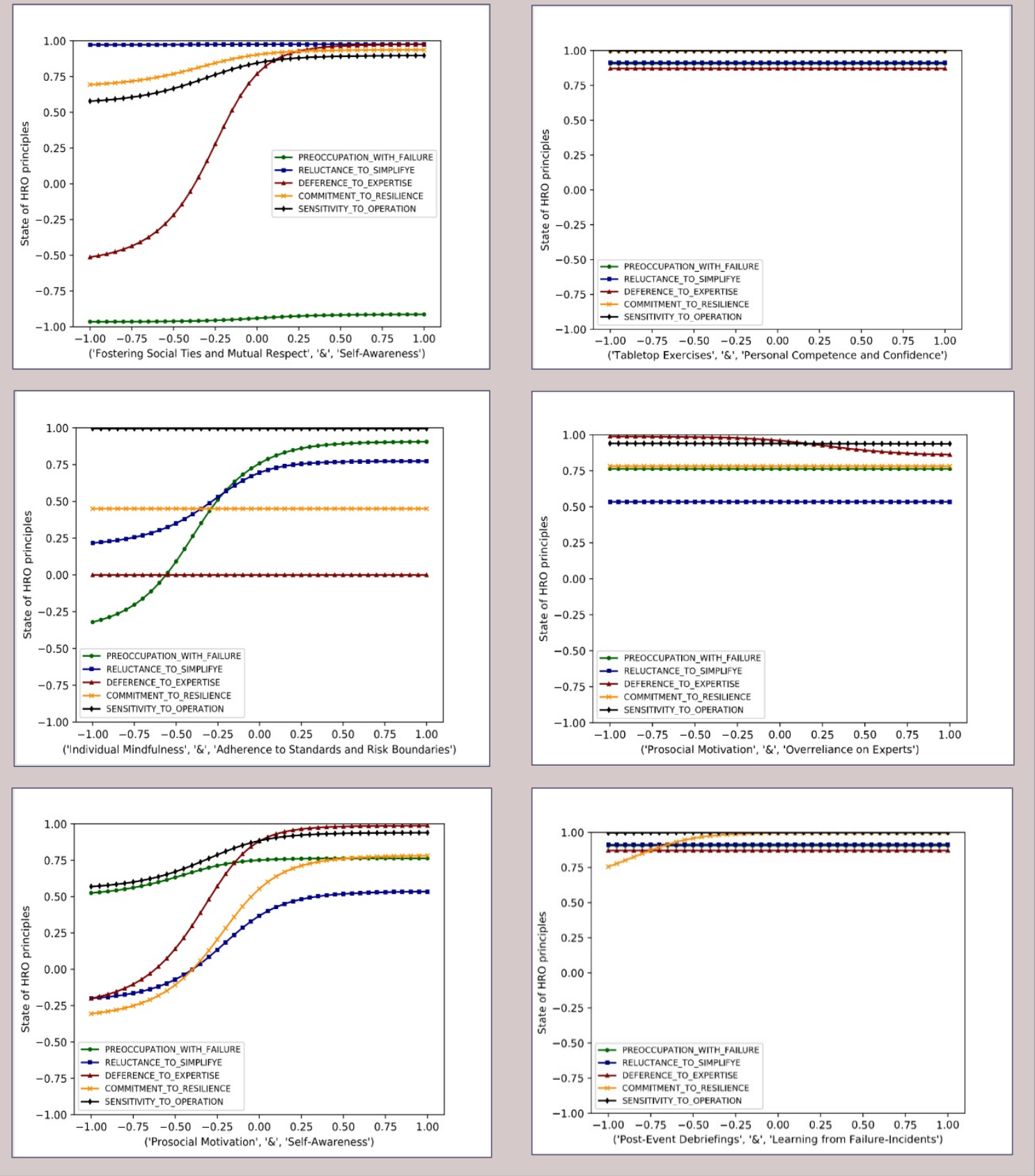

Figure 40: Sensitivity Analysis Based on Edges' Weight Variations 
Table 21: Cases Where the HROT Principles Found to Be Sensitive to Edges' Weight Variations

\begin{tabular}{|c|c|c|c|}
\hline Intervention & $\begin{array}{c}\text { HRO-Relevant Concepts That Cause } \\
\text { Sensitivity }\end{array}$ & $\begin{array}{l}\text { Sensitive } \\
\text { Principles }\end{array}$ & $\begin{array}{c}\text { Uncertainty } \\
\text { Ranges } \\
\text { [From, To] }\end{array}$ \\
\hline \multirow{6}{*}{ Stop-Work Authority } & System Flexibility and Adaptability & CTR & {$[-0.33,-0.67]$} \\
\hline & Simple Structure & CTR, RTS & {$[-0.67,0]$} \\
\hline & Structure Flexibility & CTR, RTS & {$[-0.67,0.67]$} \\
\hline & Pressure & DTE & {$[-0.33,0.67]$} \\
\hline & Level of Employee Participation & STO & {$[-0.33,0.67]$} \\
\hline & Dysfunctional Momentum & STO, CTR & {$[-0.67,0.67]$} \\
\hline \multirow{2}{*}{$\begin{array}{l}\text { Reporting of Near Misses } \\
\text { and Safety-Critical Events }\end{array}$} & $\begin{array}{l}\text { Adherence to Standard and Risk } \\
\text { Boundaries }\end{array}$ & RTS, PWF & {$[0.67,1]$} \\
\hline & Dysfunctional Momentum & STO & {$[0.67,1]$} \\
\hline \multirow{6}{*}{$\begin{array}{l}\text { Fostering Social Ties and } \\
\text { Mutual Respect }\end{array}$} & Organizational Variety & RTS & {$[-0.33,0.67]$} \\
\hline & Redundancy & PWF, CTR & {$[-0.33,0.67]$} \\
\hline & Self-Awareness & DTE, PWF & {$[0.67,1]$} \\
\hline & Ignoring Expertise & DTE & {$[-1,0.67]$} \\
\hline & Level of Employee Participation & STO & {$[0.67,1]$} \\
\hline & Self-Interest & STO, DTE & {$[-0.33,1]$} \\
\hline $\begin{array}{l}\text { Fostering a Sense of } \\
\text { Personal Accountability } \\
\text { for Safety on All Levels }\end{array}$ & Self-Interest & DTE, CTR & {$[-0.33,1]$} \\
\hline \multirow{2}{*}{$\begin{array}{l}\text { Institutionalizing } \\
\text { Prosocial Motivation }\end{array}$} & Self-Awareness & All Principles & {$[0,0.67]$} \\
\hline & Self-Interest & All Principles & {$[-0.33,0.67]$} \\
\hline \multirow{3}{*}{$\begin{array}{l}\text { Practicing Individual } \\
\text { Mindfulness }\end{array}$} & $\begin{array}{l}\text { Adherence to Standard and Risk } \\
\text { Boundaries }\end{array}$ & PWF, RTS & {$[-0.67,1]$} \\
\hline & Personal Competence and Confidence & RTS, CTR & {$[0.33,1]$} \\
\hline & Staying Focused & STO & {$[0,1]$} \\
\hline
\end{tabular}




\subsubsection{Simulation-Based on Individual Interventions}

The use of the EMA technique to simulate the model based on interventions required assigning uncertainty ranges for the weights of the previously identified sensitive relationships between each intervention and the HRO-relevant concepts. As listed in Table 21, an uncertainty range is a numerical interval representing participants' disagreements spans around a specific causal relationship and arranged from minimum to maximum in the form of [from, to] as previously explained.

Following the discussions in Section 3.6, I simulated the model following the procedure described in Approach $\boldsymbol{C}$ - Method B (see Table 7) to activate each of the interventions listed in Table 21 individually. In this process, I used hyperbolic tangent $(\tanh (\mathrm{x}))$ as an inference function, and the lambda value was set to 2 . I placed the weights of the identified critically causal relationships to the corresponding explorative ranges. The effects of each intervention on the concepts of interest are presented using a radar chart. This chart exhibits the outcomes of both the explorative and the median value so that a comparison can easily be made. Because this process generates random numbers within the explorative ranges depending on the number of the process's iterations, I conducted several experiments using the "stop-work authority" intervention, which I selected arbitrarily to determine the best random number that the process should generate to produce clearly readable outcomes. As shown in Figures 41, 42, and 43, the chart remained difficult to read until 100000 iterations were used, and obviously, more iterations beyond 100000 did not provide significant changes rather than consuming more time to produce the chart. Thus, I selected 100000 as the number of iterations in the process of simulating the model-based EMA. 


\section{Iterations}

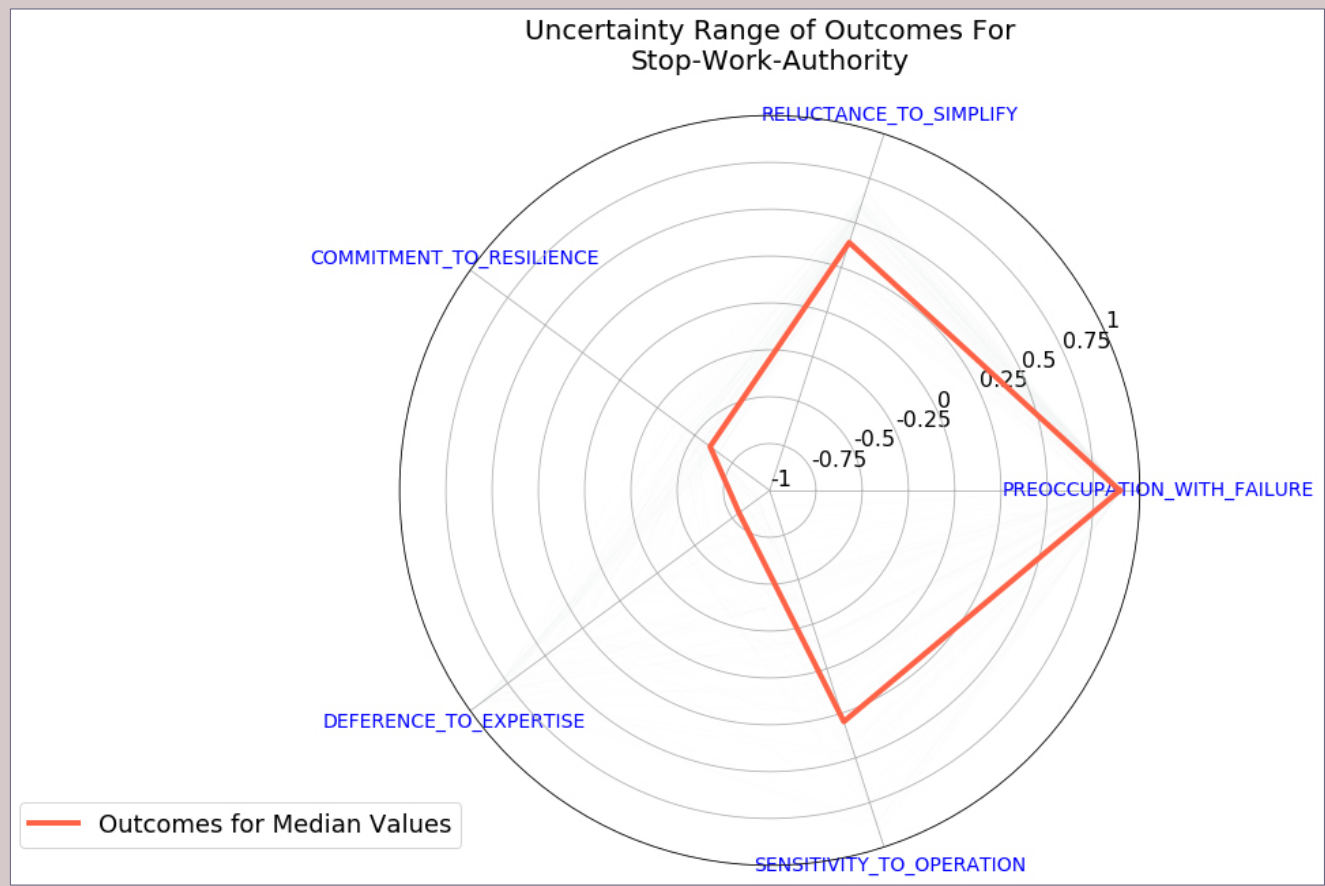

5000 Iterations

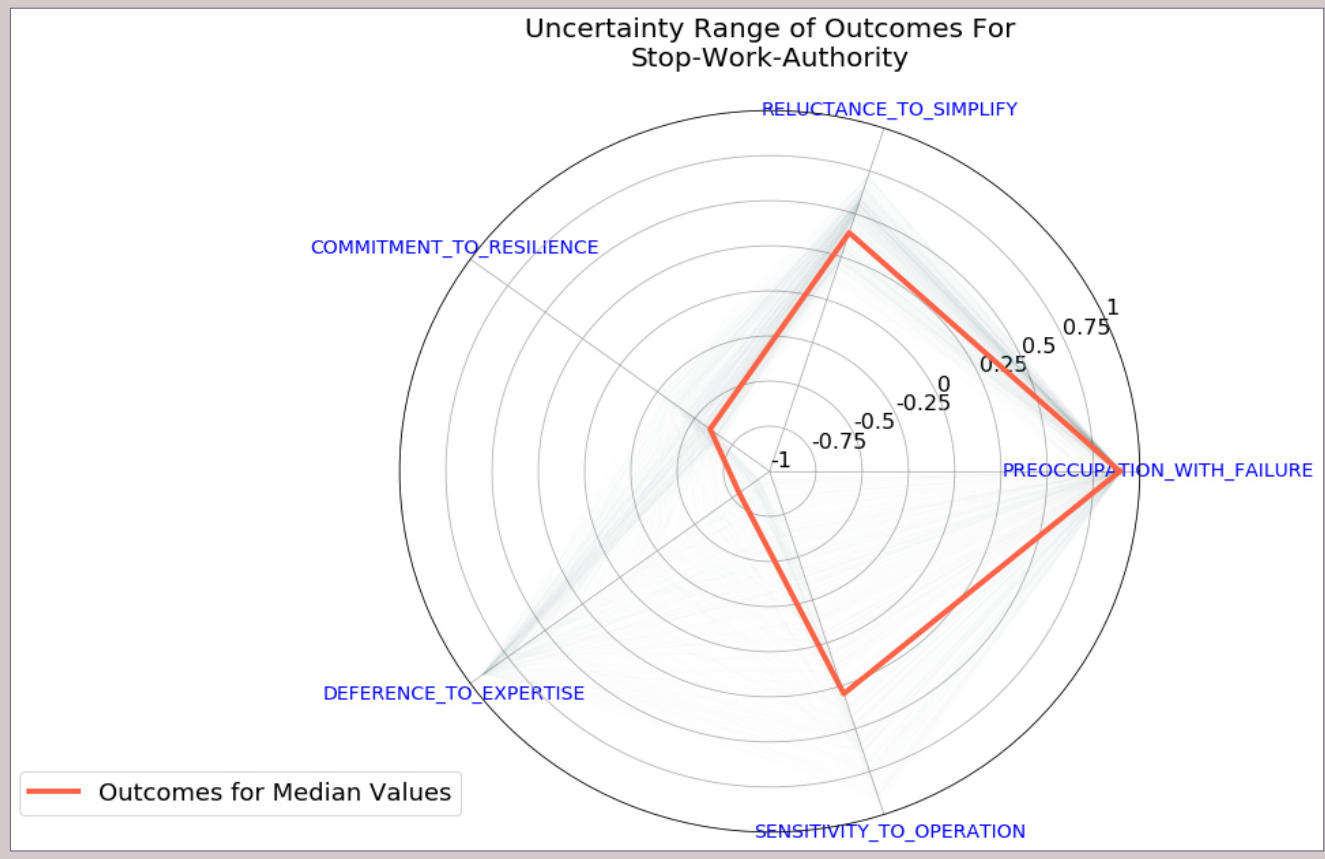

Figure 41: EMA Outcomes Based on 1000 and 5000 Iterations 


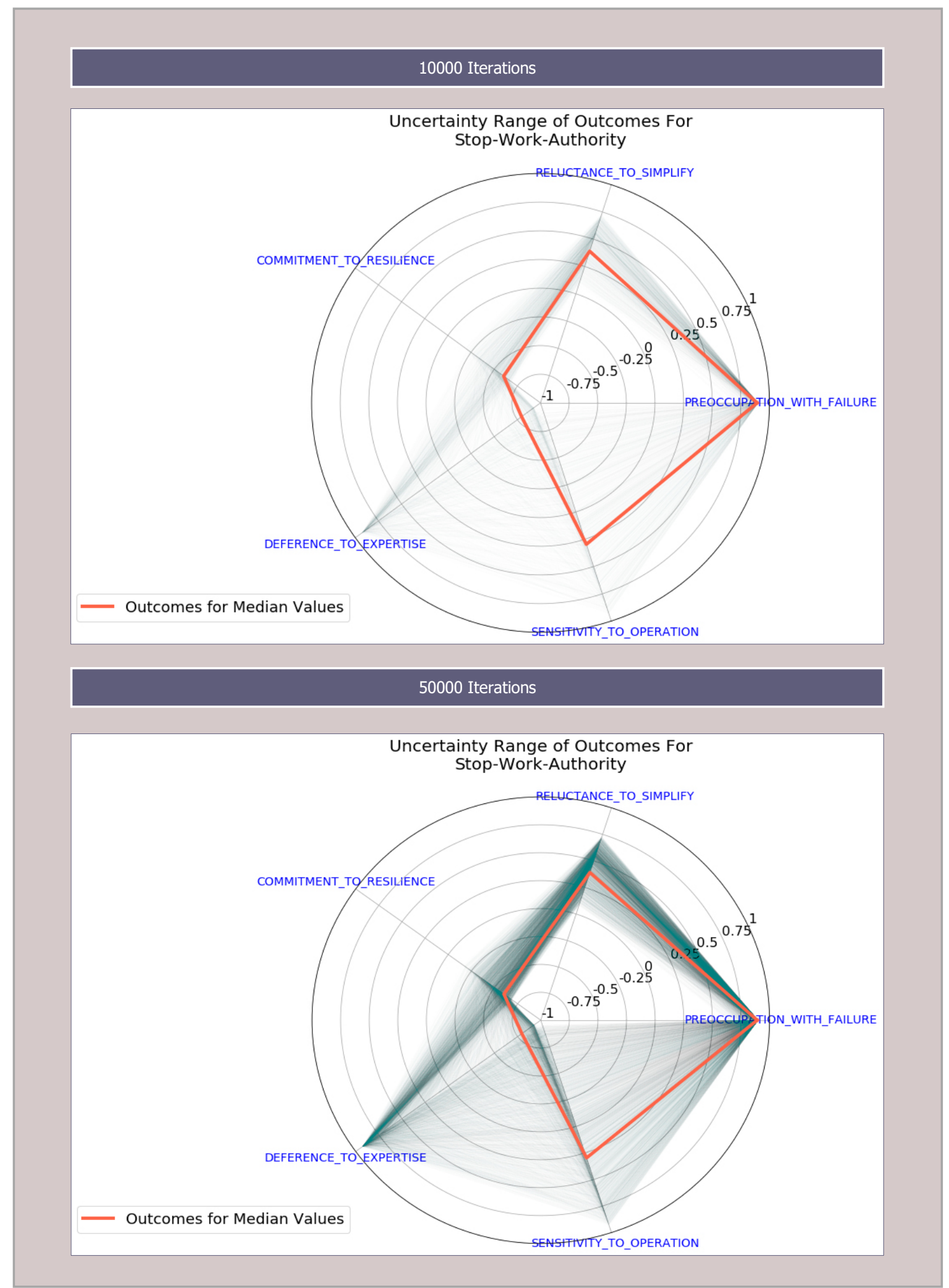

Figure 42: EMA Outcomes Based on 10000 and 50000 Iterations 


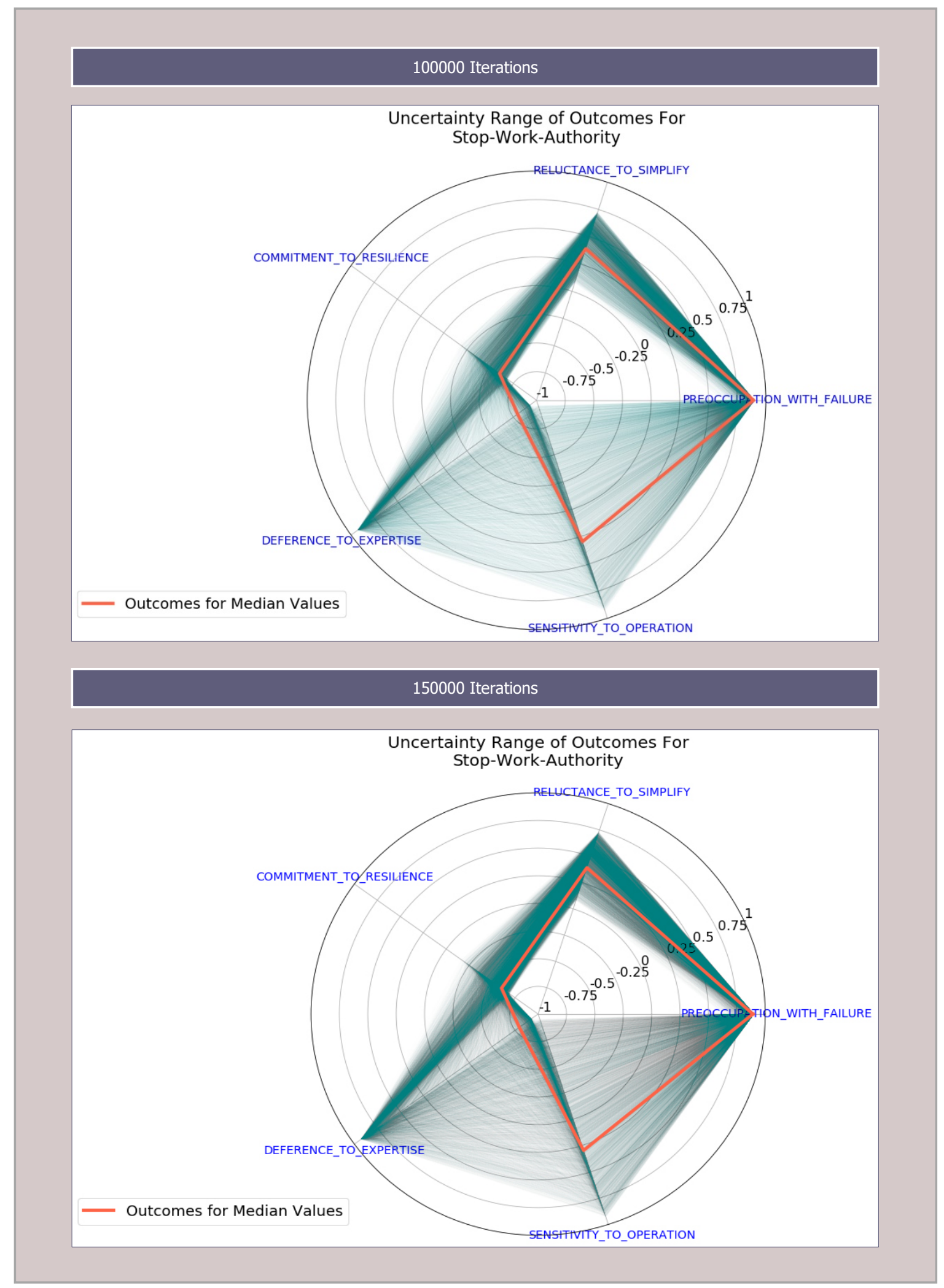

Figure 43: EMA Outcomes Based on 100000 and 150000 Iterations 
Following the entire procedure discussed above to simulate the model-based individual interventions resulted in the outcomes shown in Figure 44, Figure 45, and Figure 46. From these figures, disagreements in the participants' opinions regarding the potential influence of an intervention on the corresponding HRO-relevant concepts is not a negative management outcome. The reflection of uncertainties resulted from such disagreements can also be seen in a positive enhancement in the degree of changes in the five HROT principles. HROs always encourage contradiction spirits among the decision-makers to treat each case by its specific context. For example, in graph A - Figure 44, disagreement has expanded the possibilities of DTE to reach out with positive degrees of change and provided better outcomes of both STO and RTS. The same observations can be seen with respect to DTE and PWF in graph A - Figure 45, and DTE in graph B of the same Figure. However, disagreement impact on PWF in graph B - Figure 46 shows that PWF can be negative concerning how the decision-makers perceive the effects of practicing individual mindfulness on the workforce's focus during the normal course of operations. Negative influence can also be seen in the potentials to reach negative degrees of change in each of the five principles based on Institutionalized Precocial Motivation, as in graph A - Figure 46. More details on results analysis and interpretations are provided in Section 6.4. 


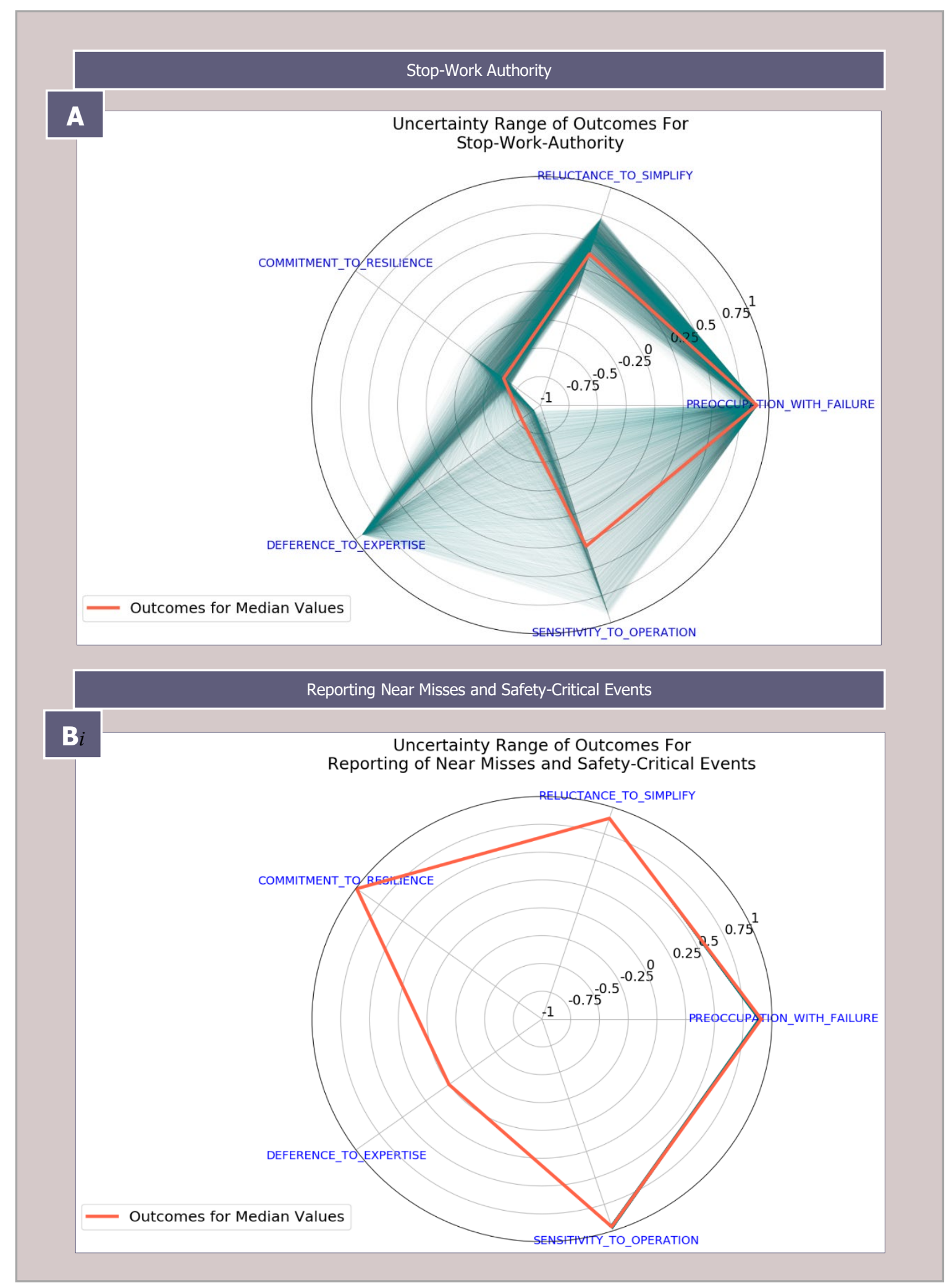

Figure 44: Effects of Individual Interventions on HROT Principles Based EMA (1) 


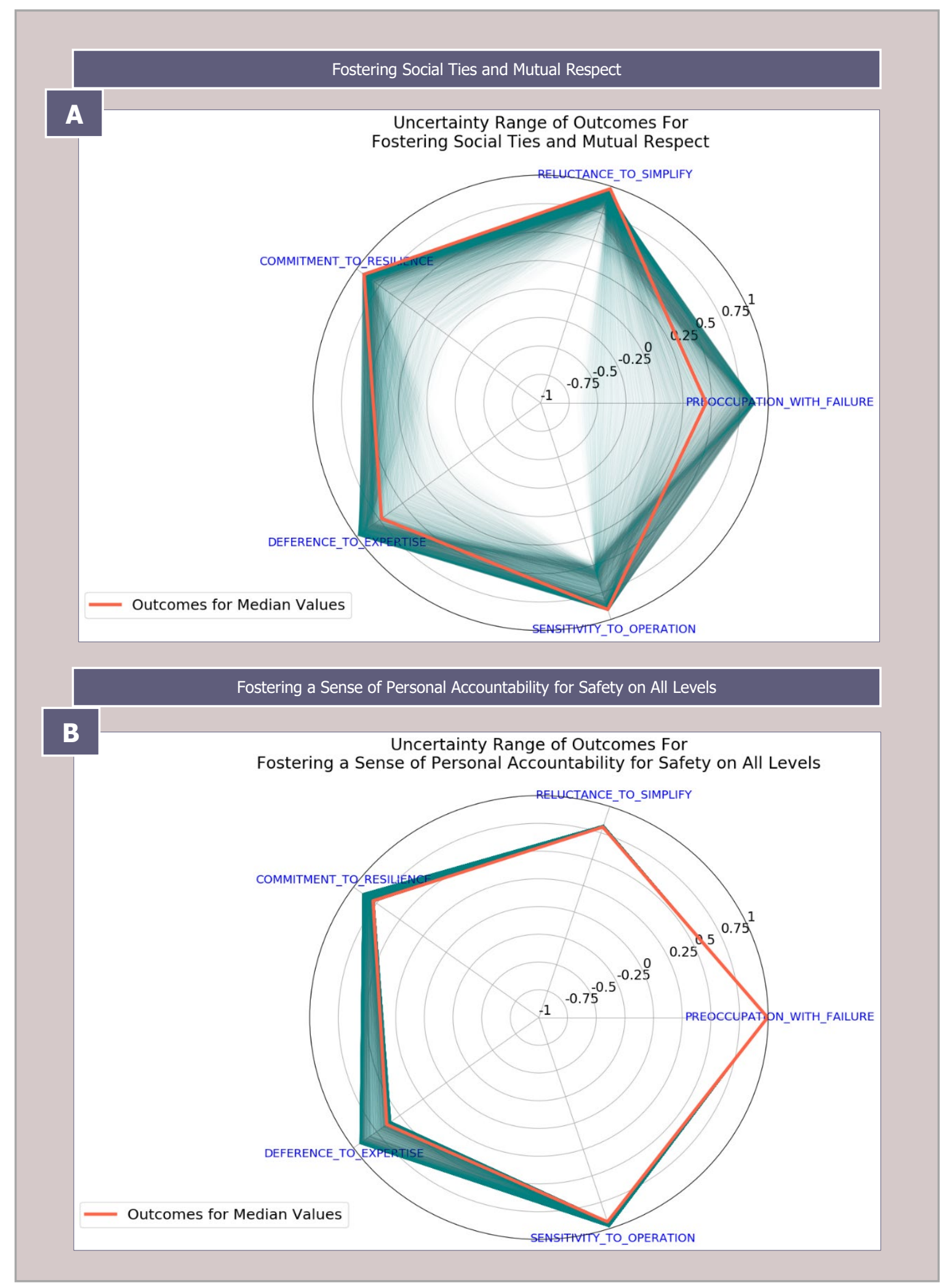

Figure 45: Effects of Individual Interventions on HROT Principles Based EMA (2) 


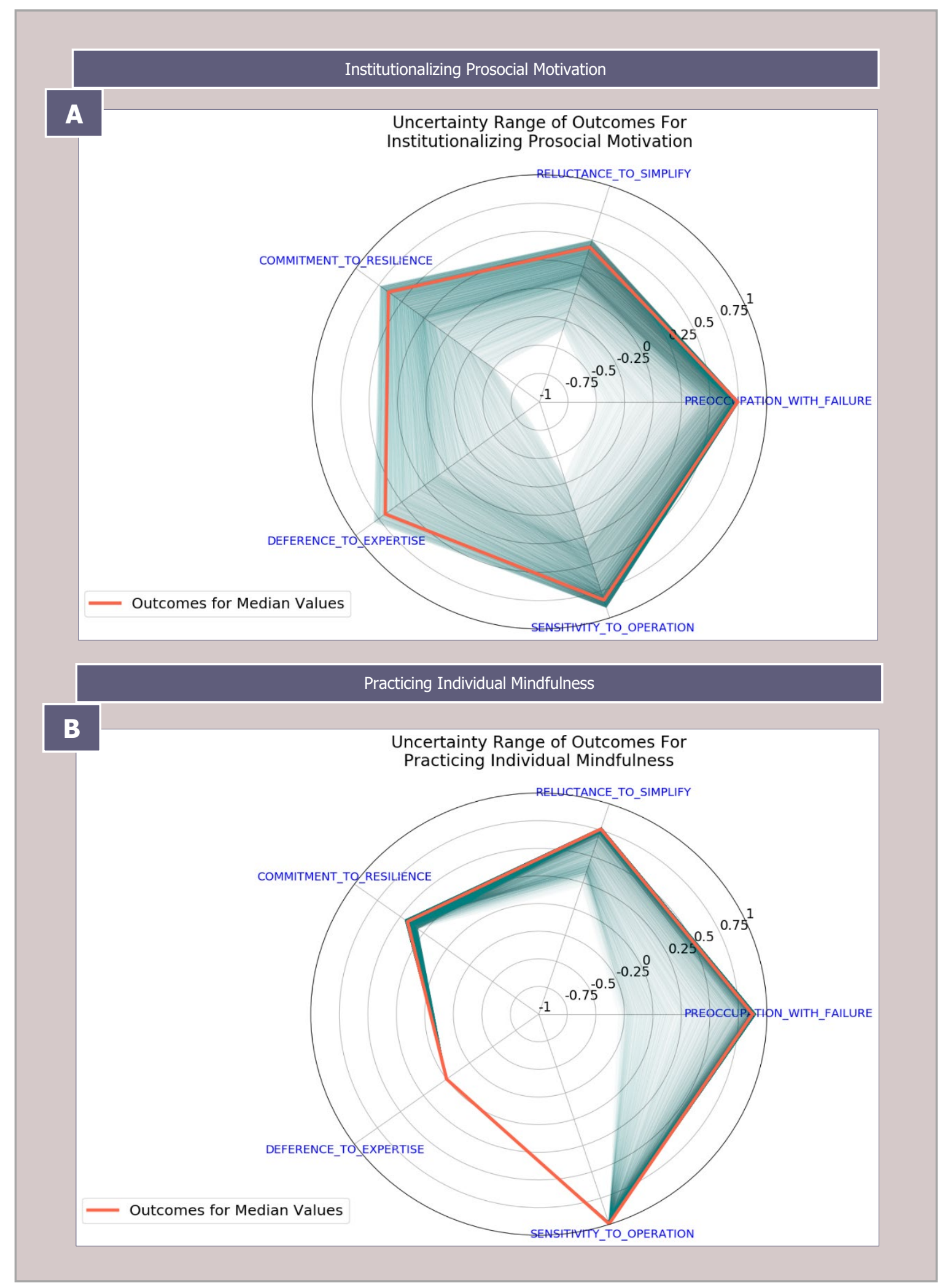

Figure 46: Effects of Individual Interventions on HROT Principles Based EMA (3) 


\subsubsection{Scenario-Based on Grouped Interventions}

I simulated the model using the EMA technique based on grouped interventions. However, only two of the five groups listed in Table 19 were used in this process: Reframing How to Relate Colleagues Peers and Reframing How the Work Should Be Done. The other groups either have no explorative ranges (see Table 21) as in the group of Reframing Work Training or have only one intervention with an explorative range as in the groups of Reframing Work Design and Reframing Work Communication. Moreover, EMA simulation does not apply to a group of best performance interventions since this type of simulation does not yield outcomes that show exact degrees of changes, but instead, it yields ranges of possibilities, which makes the differentiation between interventions based on best performance is impractical. Figure 47 shows the outcomes of simulating the model based on the two identified groups of interventions using the EMA technique. In graph A, it is evident that grouping interventions did not reduce the ranges of uncertainties. Still, it shows that Reframing How to Relate Colleagues Peers has yielded tradeoffs between the degree of CTR and the degree of STO, or in other words, the more the CTR, the less the STO. What is interesting is that the boundaries of the darker area towards the center of the graph show that PWF can go extremely negative, but this can be when RTS and STO go towards very high degrees, which is expected because redundancy comes into play when social ties are beyond the allowable limits. Moreover, graph B, Reframing How the Work Should Be Done, reflected that grouping intervention under uncertainty (i.e., diversify opinions in the right directions) could enhance what conformity (median weights) could not. More details on results analysis and interpretations are provided in Section 6.4. 


\section{Reframing How to Relate Colleague Peers}

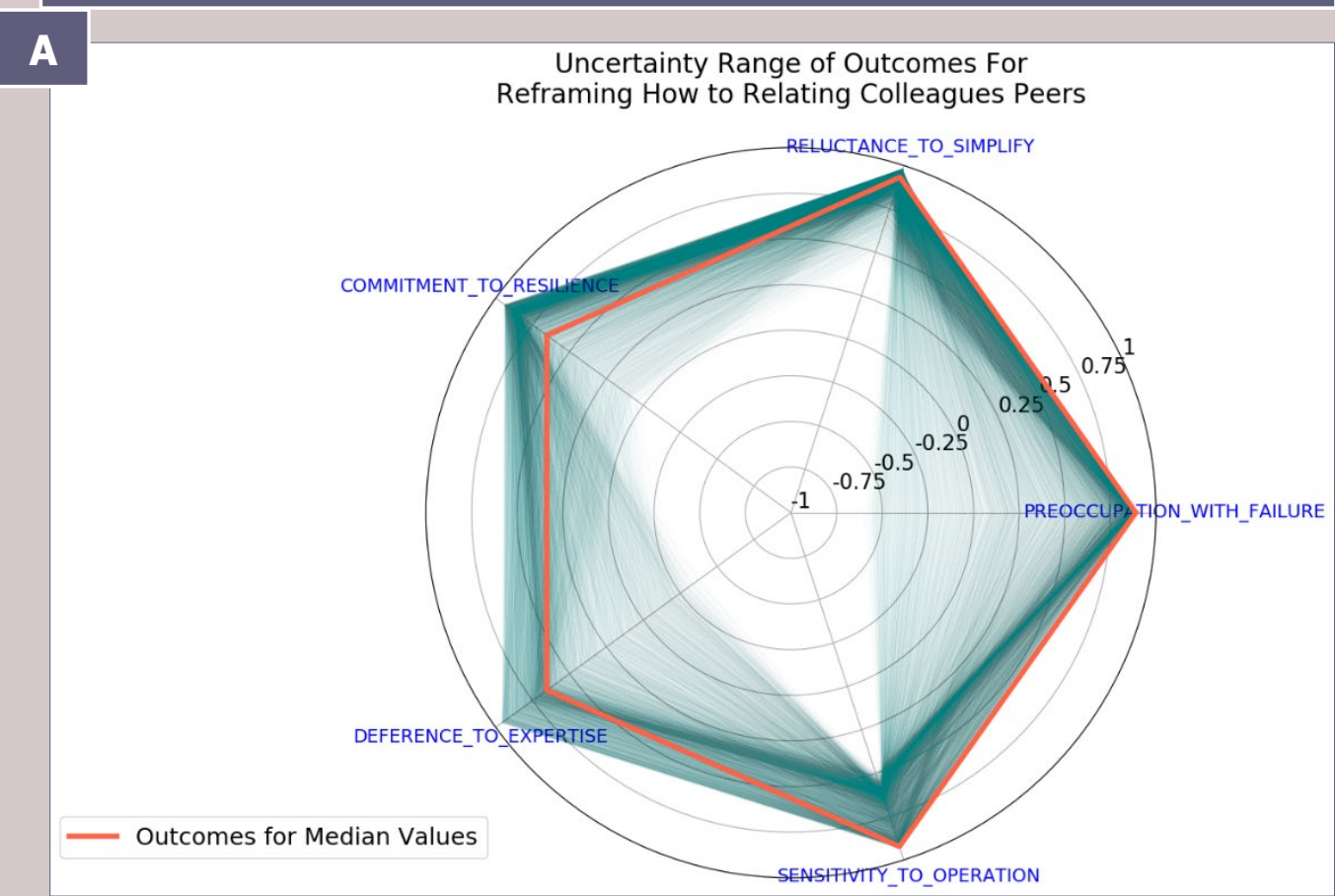

Reframing How the Work Should Be Done

\section{B}

Uncertainty Range of Outcomes For How the Work Is and Should Be Done

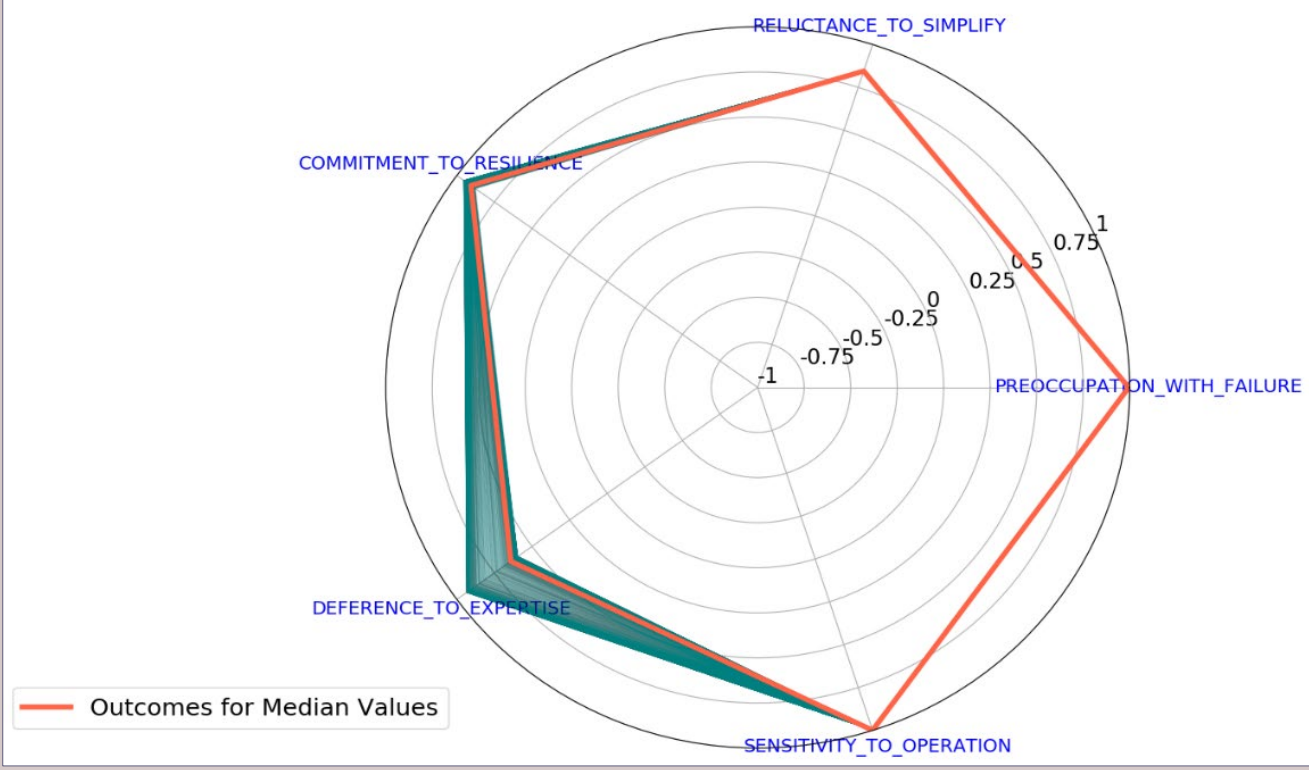

Figure 47: EMA Simulation Based on Grouped Interventions 


\subsection{Results Interpretation and Analysis}

This section provides extensive details on the interpretation and analysis of the simulation results presented in Section 6.3. At first, I provide the interpretation and analysis of simulating the model using the traditional FCM method based on the median of the collected data. Then, I discuss the interpretation and analysis of the simulation results using the EMA technique, considering participants' disagreement or the uncertainty ranges. In the second part, the analysis includes a comparison between the results of the two techniques.

\subsubsection{Results of Model Simulation Using Traditional FCM}

Based on the simulation outcomes presented in Figure 36, Figure 37, and Figure 39, as well as the numerical results provided in Table 18 and Table 20, it is evident that neither every managerial intervention (or group of interventions) impacts all the five principles at the same time nor it influences them with the same level of impact. Furthermore, all the simulation processes have been conducted by fully activating (i.e., 100\% turning on) each intervention or group of interventions; therefore, the obtained results, in most cases, present the maximum possible degrees of change in the five HROT principles as well as the overall performance represented in organizational reliability OR. However, as mentioned earlier, the maximum activation level does not always yield maximum or even desired degree of change, as shown in Figure 38, where more activation level has caused one of the principles, namely PWF, to decrease. The following sections discuss the traditional simulation process-based intervention case by case. 


\subsubsection{Stop-Work Authority}

Model simulation based on "Stop-Work-Authority" (see Table 14 for definition) shows that PWF, RTS, and STO increase due to implementing this intervention; however, CTR and DTE decrease, as shown in Figure 36. Overall, OR increases only very minimally by 0.036 (see Table 18). These outcomes occurred because the study participants assigned a decreasing relationship between this intervention and one of the HRO-relevant concepts labeled "Structure Flexibility" with direct causal connections with CTR and DTE. In particular, and according to what they have mentioned, Stop-Work-Authority as an intervention minimizes the flexibility of the system structure, which, in turn, reduces the ability to migrate the decision-making process to the frontlines in times of emergency and limits frontline worker's ability to improvise when needed. Participants perceive this intervention to stop the operations sometimes for irrational reasons instead of continuing it while coping with trying conditions as in HROs. One of the experts has commented on this intervention by the following:

"This intervention did not have a mandated directive from top management, and for that reason, there were issues with users of the facility thinking they had domain over the lower level technicians. Stop work authority can empower malicious behavior if workers feel as if they are not treated justly or are, in general disgruntled. Under ideal circumstances, stop work authority works very well."

Another participant perceived the problem of this intervention falls in two main categories:

"The issues around disruption to the business (time, cost, etc.). While these are the ones that "get the headlines," this is essentially a leadership issue more than a team issue. 
Training the right people in the right leadership skills is key to solving this type of issue, more so than any stop-work intervention. The right leaders will drive this behavior regardless of the existence of a formal "stop work authority" or not."

In general, granting Stop-Work-Authority does not improve organizational reliability in the offshore oil and gas industry and might be mismatched with prevailing work culture and management norms.

\subsubsection{Reporting of Near Misses and Safety-Critical Events}

Simulating the model based on this intervention (see Table 14 for definition) resulted in highly increasing effects on each of PWF, RTS, STO, and CTR; however, it did not affect DTE (see Figure 36). Although organizational reliability OR increased by a little over 0.750 (see Table 18), it is evident that this intervention cannot make a concurrent influence on all the five HROT principles. DTE has not changed because this intervention has no direct or indirect link to any concept that impacts the organization's structure, which is expected.

Participants based on their inputs perceive this intervention as a practical means that yields increasing impacts on HROT principles; however, they commented otherwise to explain that there is a hesitancy to report specific incidents due to fear of blame or punishment:“ There is a reluctance to report certain incidents due to fear of retribution." Another feedback summarizes the situation as: "The goal was to instill HRO principles, but it required a great deal of work to win the trust and for all employees to know they could offer input without reprisal." 
This intervention can leverage the organizations' capabilities in the offshore oil and gas industry to practice HROs positively. However, this industry needs a better strategy to promote a culture that encourages reporting all types of errors since not all organizations have such a culture.

\subsubsection{Fostering Social Ties and Mutual Respect}

Fostering Social Ties and Mutual Respect (see Table 14 for definition) has positively influenced all the five principles with varying degrees of changes between a minimum of 0.453 for PWF and a maximum of 0.977 for RTS (see Figure 37), and the overall OR has increased, by 0.797 (see Table 18). The reason behind the relatively low degree of PWF when compared to the degrees of the other four principles is that more social ties among employees can increase the overreliance on one another, which in turn breeds undesired complacency. One of the participants provided a comment claiming based on his/her experience in implementing this intervention the following:

"Social ties between employees can create ways of blackmailing subordinates through the usage of personal knowledge and pressuring. There is also the problem of being "too familiar" and no longer identifying the shortcomings of coworkers in everyday operations. This loss of critical evaluation can cause lax behavior between workers and prevent them from being significant enough to avoid short cuts".

In general, practicing this intervention resulted in promising outcomes; however, organizations must be careful because fostering social ties should not exceed the levels that can adversely affect personal accountability for safety. 


\subsubsection{Fostering a Sense of Personal Accountability for Safety on All Levels}

Simulating the model based on this intervention (see Table 14 for definition) increased the degree of all five HROT principles simultaneously, as shown in Figure 37. The degree of OR has been positively increased, by 0.831 as in Table 18 . In this particular case, the participants have shown a high level of consensus around the impacts of this intervention on the corresponding list of HRO-relevant concepts. The outcomes have presented behaviors similar to the expected behaviors, as reported in the literature on HROT.

\subsubsection{Implementing Safety Huddles}

Safety Huddle (see Table 14 for definition) provides a base for each day's communications and shifts discussions among the workforce, particularly those in frontlines. Implementing this intervention has increased four of the five HROT principles: PWF, RTS, STO, and CTR, as shown in Figure 36. The increase in the degrees of the mentioned four principles has led to increasing OR's degree by 0.757 (see Table 18). However, this intervention did not change DTE's degree, which is also expected since it has no direct or indirect link to any concepts within DTE's network. Therefore, implementing a safety huddle requires implementing other interventions that can substitute the missed effect on DTE.

\subsubsection{Practicing Tabletop Exercise, Emergency Drills, and Post-Event Debriefing}

The individual simulation of the model based on each of these training interventions (see Table 14 for definitions) increased the degrees of all five HROT principles, as shown in Figure 36. As a result, it has increased the degree of OR by 0.915 for Tabletop Exercises, 0.938 for Post-Event Debriefings, and by 0.930 for Emergency Drills (see Table 18). The positive behaviors of the principles resulting from simulating the model based on these 
interventions conclude that training the workforce is highly effective in practicing and achieving HROs. One of the participants has commented on these interventions explaining the significance of training:

"A great deal of experience in practicing emergency drills, U.S. Navy, and O\&G platforms. Tabletops do help the training but do not replace the actual exercises where you learn the most, actually going through the motions."

Another participant confirms that the offshore oil and gas industry practice emergency drills often and recommends such training for those newly employed:

"The $O \& G$ Industry drills frequently. The more experience a person has, the better they are at performing in the ways desired in a drill. For example, new arrivals aboard an $O \& G$ facility, construction site, must be oriented about the site, emergency response expectations, sounds of various types of emergencies, leadership expectations for safe work performance, etc. When a drill does occur, the expectations are familiar and may mean the difference in life or death. So, after several (or many) drills, the actions depend less on the available subject matter expert or supervisor."

There is no doubt; training is one of the most significant investments that organizations make to build their workforce's skills and competencies to practice HROs. However, the methods of training are crucial factors in how practical the training would be. As in the first comment above, virtual training is useful but cannot replace the actual training where the workforce learns most—considering that the DTE's positive degree resulted from building workforce competencies based on intensive training and practices. 


\subsubsection{Institutionalizing Prosocial Motivation}

The results of simulating the model based on this intervention (see Table 14 for definition) show increasing impacts on all five HROT principles, particularly PWF and STO, as in Figure 37, which caused the OR to present an increasing degree 0.677 as in Table 18 . The reason why STO showed the most growing degree of change when compared with the other principles is that all the participants who exercised this intervention have assigned an extremely increasing relationship between the intervention and the model concept labeled "Level of Employee Participation" that has a direct connection to STO. Participants perceive this intervention as an approach to develop confidence and trust in the corporate vision as one of the participants has pointed:

"The company has offered soft training for staff leadership, like your Pro-Social Motivation. The immediate impact was increased understanding and trust in the corporate vision."

Practicing this intervention is highly recommended because it is very well aligned with the trust that HROs rigorously build. Although this intervention has no direct impact on RTS, however, RTS showed an increasing degree, which resulted as a side effect (desired) caused by the causal relationship with concepts in the other principles networks that have direct links with the intervention.

\subsubsection{Institutionalizing Emotional Ambivalence}

It is evident through Figure 37 that simulating the model based on this intervention (see Table 14 for definition) has resulted in increasing degrees for all the five HROT principles. As a result, the degree of OR increased by 0.893 (see Table 18). This outcome is predicted 
since participants' inputs have met the expectations regarding each causal relationship between this intervention and the list of the corresponding HRO-relevant concepts.

Indeed, emotional ambivalence promotes a culture that motivates the workforce to believe in their competencies and skills as they operate safely; however, they remain skeptical that issues can occur anytime during the normal course of operations, and despite their competencies, they do not mind to enquire information from more skilled people in the organization.

\subsubsection{Managing Reliability Drift}

This intervention (see Table 14 for definition) is majorly focused on enhancing the practice of STO, which has been achieved (see Figure 36), with an increasing degree of 0.775 (see Table 18). While both PWF and RTS also resulted in increasing degrees, this intervention had no effects on both CTR and DTE and, therefore, the overall OR is 0.330. However, one of the predictions is that this intervention would increase the relationship with DTE since when the operations procedure drifts, the decision-making process shifts to those who know the context of the problem better to fix it without turning the operations off. The participants decided that there is no relationship with DTE with no comment on why they chose so. Perhaps and according to the following statement by one participant, they might have considered drift can occur in human behaviors, not in the technical procedure: "Managing Reliability Drift, as described, is managing human behavior by coaxing humans to "be better." I've never viewed this type of management as sustainable. Managing Reliability Drift that focuses on enabling humans to make the reliably safe and correct work decisions easier than making the decisions that cause, enable drift." 
With this comment, it is evident that this intervention is essential but requires a clear definition so that people can understand the meaning behind it. This might be investigated in future research.

\subsubsection{Practicing Individual Mindfulness}

Practicing individual mindfulness (see Table 14 for definition) is designed to help people in the front line, particularly operators, prepare themselves mentally before starting their

daily tasks. They can stay focused on work activities. Thus, they maintain a high level of STO. The simulation results show that STO's performance is increasing (see Figure 37); however, the results also show no impact on DTE and low degrees of CTR, PWF, and RTS when compared with STO. In general, the simulation process yielded an increasing degree of OR by 0.61 (see Table 18). One of the participants has commented on the following: "Practicing Individual Mindfulness is a great deal like being a violinist in a symphony. It requires daily practice and also sharing the practice with the people in your sphere of influence (the symphony members, one's family, and one's church and civic organizations. $A S$ the practice becomes a habit, the people around you each day are positively influenced."

With respect to the definition of this intervention and how the practice has been proved to be an effective means to help frontline workers stay focused on their duties, this intervention does not increase all the five HROT principles simultaneously.

\subsubsection{Adopting Just-In-Time Learning}

From the results of simulating the model based on this intervention (see Table 14 for definition), it is evident that the decreasing effects on four of the five HROT principles: 
PWF, RTS, CTR, and STO (see Figure 36) conclude the undesirability of applying this intervention in real-world operations. These four principles' decreasing degrees resulted in an overall degree of OR below zero by 0.750 (see Table 18). Industry experts perceive justin-time learning as a distracting means instead of a training or communication method to inform people in real-time about issues happening in the process. According to one of the experts, this intervention can cause a problem with workers' skills:

"The oil and gas industry is very training intensive and the offshore roles, in particular, demanding. A system of just in time learning would create challenges in the delivery, but timing and location, as well as issues with a competency to be deployed."

An intervention is typically designed and implemented to positively influence an organization's total performance, particularly in terms of safety; however, when an intervention is tested, and the results are mostly counterproductive, the intervention should not be practiced, especially in this industry.

\subsubsection{Grouped Interventions}

Simulating the model based on groups of interventions (see Table 19), rather than individual interventions, showed that each group has achieved improvements in most or all of the five HROT principles but affected the principles differently, as shown in Figure 39. In one instance (i.e., interventions targeted at reframing work communication - graph C), the model simulation shows that this group of interventions led to a substantial increase in four of the five principles (PWF, RTS, CTR, and STO); however, this group did not change DTE. Moreover, in three instances, namely the group of interventions for reframing work design (graph A), reframing how to relate colleagues peers (graph D), and reframing how 
the work is and should be done (graph E), simulations identified one or two HROT principles that showed a much smaller increase than the other principles. This indicates that focus on single groups of interventions (e.g., an initiative solely focused on communication or relationships between employees) will leave some essential HROT principles relatively or entirely unaffected. However, one exception is the group of interventions designed to train employees (graph B) based on both actual work training and training by simulation. These measures are found to have a strong positive influence on all five HROT.

Apart from training, none of the groups of interventions is capable of producing strong improvements across all HROT principles, which limits the possibility of achieving organizational reliability, particularly if OR turns out to be determined by the weakest contributing principle. Accordingly, focused company initiatives for improving HROT principles should be mindful of where they will fall short and consider adding interventions specifically designed to support the HROT principle that the initiative fails to sufficiently improve.

From a modeling and practical perspective, this raises the question if it might be possible to design a program for organizational reliability that selects the best intervention from each group of interventions and what magnitude of improvement it would generate. Simulation results (presented in graph F) show that this strategy would result in a very pronounced improvement of all HROT principles - the best intervention from each group thus appear to be synergistic enough to deliver results that outperform those of any single 
group of interventions. A focus on these interventions may allow companies to cover all areas with relatively limited effort.

\subsubsection{Results of Model Simulation Using Explorative FCM (EMA Technique)}

This section covers the analysis and interpretations of the EMA simulation results based on individual and grouped interventions presented in Figure 44, Figure 45, Figure 46, and Figure 47. As provided in Table 21, the EMA simulation was done for only six interventions. All the simulation processes using EMA have been conducted by fully activating (i.e., $100 \%$ turning on) each intervention or group of interventions so that the obtained results, in most cases, present the maximum possible degrees of change in the five HROT principles as well as the overall organizational reliability OR. However, as mentioned earlier in traditional simulation, which is also observed in the EMA simulation, the full activation level did not always improve performance. In some cases, it has yielded counterproductive effects.

As discussed earlier in Section 6.3.2.2, each time the model is simulated based on EMA, the simulation process was iterated 100000 times, and each time the process was iterated, a random number is generated within the uncertainty range as an edge weight that results in an impact on one or more of the five HROT principles. The effect is plotted as a thin line on the radar chart to represent the behaviors of the principal(s) under the impact. When the same or a close random number is generated as another edge weight (the subsequent iteration), its influence is plotted over the first line or closely next to it. Therefore, a thick line or a dark shaded area on the radar charts infers that a large portion of the generated random numbers (i.e., edges' weights) was auto-selected to be either the same or very close 
to one another within the uncertainty range. These thick lines and shaded areas on the chart are given considerable attention when the simulation results are analyzed and interpreted.

\subsubsection{Practicing Stop-Work-Authority}

Based on graph A in Figure 44, although the ranges of possibilities have been expanded to include better degrees of change in each of STO, DTE, and RTS as well as a relatively small increase in CTR, however, CTR still presented a negative degree of change, which can be seen in the range between -0.75 and -0.25 . These results validate the participants' notion regarding the contradiction that this intervention can create by decreasing the extent of the structure's flexibility and workforce capabilities to make the required improvisation when is needed since this intervention stops the operations rather than continuing them while coping with issues when occur.

\subsubsection{Reporting of Near Misses and Safety-Critical Events}

Simulating the model based on this intervention has shown identical results to the median (see graph B in Figure 44). The explanation for this situation is that the uncertainty ranges of the edges' weights between this intervention and the two HRO-relevant concepts are the same $[0.67,1]$, as listed in Table 21 . These ranges are relatively small and within the same sign direction (i.e., positive). This infers that all the generated random numbers were within and around the median value of the participant's inputs. However, even with these minor uncertainty ranges, maximizing the implementation of this intervention cannot ensure the synergetic practice of HROs, since the degree of DTE will always be zero (i.e., no effects on DTE). This means that other interventions must be implemented together with this intervention to ensure that all five principles are influenced concurrently. 


\subsubsection{Fostering Social Ties and Mutual Respect}

Uncertainty existed concerning the weight of connections between this intervention and six HRO-relevant concepts. Graph A in Figure 45 shows that this uncertainty has little effect with respect to the outcomes of RTS, CTR, and STO. EMA simulation shows that these concepts increase for all possible model structures and increase close to the broadest possible increase for a large majority of model structures. Thus, the results largely mirror those of simulating the model based on the median edge weight (indicated by the orange line). However, for STO, a large number of models lead to a lower increase than the conventional FCM model. Nevertheless, given the uncertainty, the intervention is a robust means to improve these three principles.

With regard to PWF and DTE, most models show an increase over the increase achieved in the conventional FCM model (orange line), meaning that the uncertainty of edge weights leaves room for the possibility that the intervention might actually be more effective than what the conventional model assumes. However, there is one important caveat: In some models, PWF decreases as a result of the intervention. From a practical point of view, this means that managers cannot be entirely sure that "fostering social ties" does not backfire and have unintended negative consequences. This speaks to the need to understand better the dynamics that could cause such an effect and resolve uncertainty, carefully monitor PWF, and design interventions focused on social ties while also emphasizing PWF.

\subsubsection{Fostering a Sense of Personal Accountability for Safety on All Levels}

There exists considerable uncertainty with regard to the weight of the relationship between the intervention of "Fostering a Sense of Personal Accountability" and the causally 
connected concept "Self Interest," With a range of [-0.33, 1], the participant could not even agree on the sign. However, the results of simulating the model using EMA are similar to those from the simulation process using traditional FCM with an edge weight based on the median (see graph B in Figure 45). Indeed, the degree of change for PWF, RTS, and STO is indistinguishable from a simulation-based on median edge weights, which is expected since only DTE and CTR have shown sensitivity to the variation in the weight of this critical relationship. For DTE and CTR, the outcomes in response to different edge weights show that the intervention achieves improvements under all tested edge weights, and even for the worst case, the improvement is still 0.65 of the best case. Accordingly, the intervention is a robust strategy that is advisable even under existing uncertainty.

\subsubsection{Institutionalizing Prosocial Motivation}

This intervention encountered uncertainty around the relationships with two HRO-relevant concepts: Self-awareness and self-interest (see Table 21). The range of uncertainty around the relationship with self-interest is relatively broad [-0.33, 0.67], which explains that the participants disagreed even on the sign of the relationship and because this concept has indirect relationships with many concepts in the other principles networks, thus, simulating the model based on this intervention has resulted in a wide range of possibilities in the degrees of change in all the five principles. The ranges of degree possibilities vary from negative to a little beyond the positive median line. Based on what graph A in Figure 46 shows, there can be four possible interpretations for the simulation results:

1. The achieved degrees are the same as or little over the median of the edges' weights, which means between a little over 0.40 and 0.90 . 
2. The achieved degrees are represented in the darker line between 0.00 and 0.25 , although they are low to slightly increasing degrees within a positive range.

3. The achieved degrees are within the shaded area between the median and second thicker lines, which also provides positive yet low degrees.

4. Degrees within the decreasing (negative) area.

This concludes that although disagreement (contradiction spirit) of decision-makers in HROs are practically encouraged and welcomed, it is in some cases can lead to a wide range of uncertainty when it spans over a broad range of disagreements such as the one mentioned above. When there is another range of uncertainty (i.e., another critical edge weight), the outcomes can be a long list of possibilities.

\subsubsection{Practicing Individual Mindfulness}

Three critical causal relationships between this intervention and HRO-relevant concepts encountered disagreement in participants' opinions (see Table 21). Despite that the uncertainty range around one of the relationships is very large $[-0.67,1]$, simulating the model based on this intervention resulted in outcomes that consist of degree possibilities mostly around PWF, by a broad range $[-0.50,0.90]$ and RTS, by slight $[0.35,0.75]$. However, most, if not all, the iterations considered numbers within the median range of the edges' weight are clearly presented in graph B Figure 46 . By viewing the possibilities that match the median line as the maximum attainable degrees, this intervention remains has no effects on DTE since there are no direct and or indirect relationships with any concepts within the DTE network. Furthermore, even when the implementation of this intervention is maximized, which already has been, the degree of CTR cannot be increased beyond 0.50 
because there is no direct relationship between the intervention and any HRO-relevant concepts within the CTR network.

\subsubsection{Grouped Interventions}

As mentioned earlier, the process of simulating the model as EMA based on groups of interventions included the use of only two of the five groups listed in Table 19: Reframing how to relate colleagues peers and the group of reframing how the work is and should be done. These two groups consist of interventions that encountered uncertainties, or in other words, participants' disagreements. The following discussion analyzes and interprets the results of the simulation processes of these two groups.

Concerning the first group (reframing how to relate colleagues peers) as in Graph A in Figure 47, EMA simulation based on this group resulted in ranges of possibilities:

1. It mirrored the degrees of change based on the median of the data for each of RTS, CTR, and STO.

2. It achieved higher (preferable) degrees beyond the data median for both PWF and DTE.

3. The graph shows that when the degree of PWF is within the maximum range of possibilities (i.e., between 0.75 - nearly 0.85 ), the degree of STO can be below the median line (i.e., between 0.50 - nearly 0.75 ), which at the same time increased the degree of DTE.

This also concludes that simulating the model based on groups of interventions using EMA enables practitioners to understand how their decision-making, including their disagreements, can yield tradeoffs. In the best decision scenarios, practicing HROs can 
never deliver equal outcomes for all the principles. However, this group of interventions has positively influenced all the five principles at the same time.

Furthermore, simulating the model based on the group of reframing how the work is and should be done yielded degrees for all the principles that paralleled the degrees resulted from simulating the model based on the data median (see graph B in Figure 47). The graph shows that the simulation yielded an increasing range of degrees for DTE beyond the median line. There is one possible interpretation of why the simulation did not result in ranges of the degrees for PWF, RTS, CTR, and STO, like DTE, is that each of PWF, STO, and CTR already reached maximum degrees based on the median. No range was left beyond the median, and because RTS has no direct effects on the grouped interventions, the simulation did not generate a range of degrees beyond the median. However, it is sensitive to the variations in the relationship between these interventions and the HRO concepts (see Table 19). Because the second intervention directly affects DTE with the most extreme range of uncertainty $[-0.33,1]$, the simulation resulted in a range of degrees for DTE. 


\section{Summary and Discussion of Results}

In this chapter, I step back from the detailed results described above to draw conclusions. In the following Section 7.1, I will compare and contrast the results of my different analyses to distill the gained insights with regard to two topics - what I learned about HROT principles as a system and what I learned about practical needs and means to improve safety-related practices in the offshore oil and gas industry. In Section 7.2, I will review the research questions underlying this work in light of these findings.

\subsection{Summary of Results}

\subsubsection{HROT Principles as a System}

High Reliability Organizations Theory (HROT) has been developed by studying real-world systems of interdependent and causally related cultural elements (concepts). My work, for the first time, makes this system perspective explicit by modeling the principles tenants of this theory with FCM. The resulting model suggests, not surprisingly, that all the five HROT principles are contributing to developing organizational reliability (OR) and that they are interrelated because they affect, and are affected by, many of the same concepts. Works on HROT usually consider the principles to be synergistic ("a package") and recommend improving all of them to the furthest possible extent. However, this research shows through FCM modeling and simulation that few managerial interventions can simultaneously improve all five principles (i.e., achieving the package) and that improvements in some principals can impair others' implementation. A case in point is simulating the model for "Stop-Work Authority" (see graph A-Figure 44) and "Fostering Social Ties and Mutual Respects" (see graph A-Figure 45), which resulted in worsening 
of some principles. Moreover, there have been several cases where model simulations show that increasing the activation of a concept (e.g., from 50\% to 100\%) does not yield any further improvements in desired outcomes but may actually worsen them, as shown in Figure 38 .

The common recommendation to work towards improving all HROT principles is thus, most undoubtedly wrong. At best, it is inefficient because it causes organizations to invest in interventions even after there are no marginal returns anymore. In many cases, it may even be detrimental to the desired results. Instead, organizations need to carefully select a suite of interventions that are designed to work together. My investigation of grouped interventions shows that selecting a few effective interventions (one from each major type, such as work design, communication, human relations - see graph F in Figure 39) yields very high improvements, whereas focusing on interventions in just one area frequently leads to limited or no improvements of some principles (see few cases in Figure 36 and Figure 37). In other words, not surprisingly, there is no silver bullet either in the form of a single intervention or a focus area for interventions that improve the system regarding all the five principles. The only exception is management actions for training the workforce (see Figure 36, and graph B in Figure 39), which have led to achieving the package. Training not only leveraged the workforce competencies to cope with the unexpected but also strengthened their culture to practice what prevents the unexpected from occurring. The package also has been achieved when management intervened to develop a culture that both motivates the workforce and emphasizes accountability for safety at the same time (i.e., the case of "Emotional Ambivalence" see Figure 37). 
Perhaps even more important, FCM modeling and simulation demonstrated what - maybe counterintuitively - does not work as a means for improving HROs practices. Figure 36 shows the simulation results based on two interventions where communication is the main practice: "Safety Huddles" and "Reporting near-misses and safety-critical events." They resulted in very similar performances; however, no effects on DTE. Even when grouping these two interventions into one group, the results were similar (graph C in Figure 39). Though good communication is regularly mentioned by practitioners (see comment in Section 6.4.1.2) as a means for improving safety (and sometimes treated as a silver bullet), communication-focused interventions do not result in improvements across all the five principles.

Similarly, there currently is some enthusiasm for (but also criticism of) just-in-time learning, live communication and reporting, and other in-time updates because it seems easier to motivate employees to learn and retain information when there is an actual need for information. The results of the simulation process show such methods to be counterproductive. This is in line with some of the expert' opinions provided in the survey, namely that just-in-time learning through computer screens or other gadgets can be distracting rather than informative and that employees, particularly operators, must focus on their task, stay sensitive to the operations, and always be cautious and alert.

\subsubsection{Model for Decision Support and Training}

The discussion above shows that decision-makers in companies need to carefully select a combination of interventions and evaluate their indirect and potentially unintended consequences, which requires some form of decision support. The innovative approach 
designed and implemented in this research delivers just that, namely a carefully designed and tested decision support system (DSS) that aids organizational decision-making regarding safety-related practices. Management in any industry, including the offshore oil and gas, can use this simulation model (i.e., FCM based HROT) to (1) Understanding the effects of the management actions on safety-related practices and (2) testing the feasibility and effectiveness of designing interventions, strategies, and policies before practically implement it. As decision support, this model enables the decision-makers to simulate scenarios through which they understand and predict how their assumptions and current and planned structural designs, strategies, and behaviors impact HRO principles. Scenarios can be any internal change (i.e., within the core model) or external change (i.e., new strategies or interventions that affect the core model's concepts).

The FCM model is likely also suitable as a training platform, similar to "microworlds" or "management flight simulators" developed in business (Papageorgiou et al., 2008), where decision-makers get to experience the impact of their actions through simulation. This is particularly promising when coupling the model with learning approaches for safety cultural training. Simulation-based training, such as tabletop exercises, are actually quite common in the industry (see comment by the practitioner in Section 6.4.1.6). I modeled three training interventions (emergency drills, tabletop exercises, and post-event debriefings). They have strong positive effects on all the five HROT principles, ranging from 0.79 to a maximum of 0.99 . This shows that: (1) Training is highly influential and continuously essential for safer operations, and (2) practitioners from the offshore oil and 
gas share a similar mindset regarding training, making an FCM-based training platform, based on the model developed in this dissertation worth pursuing.

\subsubsection{Diversity of Perspectives}

Safety culture research investigates the complex, challenging to observe systems with limited data and long timeframes. It inevitably must rely on the expertise and opinion of the people involved in the system, such as operators and safety engineers. This work makes an essential contribution to this practice: it has shown that EMA is a practical means to overcome uncertainties resulting from disagreements and variations in the experts' opinions. The experts' opinions collected through the online survey comprised wide ranges of disagreements or uncertainties regarding specific relationships between the applied interventions and HRO-relevant concepts. These disagreements are normal outcomes for diversity in personal opinions, experiences, duties, and educational backgrounds. Disagreements are, in fact, aligned with the organizational variety and contradiction spirit that the HROs built as a groundwork for redundant and extensive deliberation when issues occurred. To overcome high levels of conformity that leads to the undesired simplification and normalization, HROs encourage contradiction with the goal to enhance the performance of the five principles, particularly RTS. Rather than forcing people to agree on a single perspective and model, EMA preserves these disagreements and plots a range of possibilities. Instead of only one structure (e.g., one crisp weight of the causal relationship), EMA models thousands of alternative structures, which are visualized. This has several benefits. First, for EMA to be practical, I had to identify those edges that mattered for the model outcomes overall versus those that did not impact the system's 
dynamic. This helps decision-makers to focus their attention only on disagreements and uncertainties that matter. Second, in many cases, EMA shows a range of models that predict better performance with regard to HROT principles than the "crisp," traditional FCM model, such as in graph A in Figure 44, graph A in Figure 45, and graph A in Figure 46. This can encourage action in a situation in which many teams do not act, namely when they disagree and when things are uncertain. (Of course, the opposite, namely a large number of EMA models that show worse results than the traditional FCM model, are also possible, though this case did not occur in my study). And third, EMA helps decision-makers understand that even a relatively small difference in an edge weight, if occurring at critical edges, could result in a large change on the system level. This not only sensitizes them to appreciate the dynamics of the system but also helps them determine which uncertainties or disagreements are worth resolving through further discussion or research and where they need to pay attention during the implementation of decisions.

\subsubsection{Industry Insights}

The participants from the offshore oil and gas who took part in this research through the workshop and the survey have explained the logical reasoning behind their assignment of causal connections and causal weights (see Section 5.3.3). They also made comments that help interpret the simulation results (see Section 6.4.1). These comments reflected on the existing situation regarding safety performance and culture in this industry and, together with the simulations, provide industry insights: 
1. Stop Work Authority: According to the study's participants, when organizations authorize people to stop-work ${ }^{24}$ whenever and wherever it is needed, people in upper levels may overestimate their granted authorization. Accordingly, they may excessively dominate the workforce in the lower levels, and as a result, they could negatively affect the level of their participation and commitment to resilience. This evidently has been observed in the results of simulating the model based on this intervention with respect to the experts' opinions as it has negatively affected the performances of CTR, DTE, and STO, which seamlessly explains this cultural shift.

2. Reporting Events: Fearing punishment for reporting near-misses and safety-critical events prevent safety culture from emerging. The people involved in the operations lack information that is critical for them to become aware of what is happening and how to resolve issues. On the flip side, encouraging the reporting of near-misses and safetycritical events can lead to a significant improvement in the performance of each of PWF, RTS, CTR, and STO, as shown in the simulation results in Figure 36. However, it is not a silver bullet for achieving safety culture because it did not improve all five principles simultaneously (i.e., achieving the package).

3. Fostering Social Ties: It has positive impacts on the performance of HROT principles. However, excessive social ties among colleagues can result in a cultural tendency of

\footnotetext{
${ }^{24}$ Based on a conversation with an expert from the offshore oil and gas industry regarding the use of this intervention, stop work authority has become a double-edged sword. While the use of this intervention has been emphasized since 2013 when BSEE has reinforced not only authorizing any offshore personnel who witness an imminent risk or dangerous activity to stop the work but also to make them responsible for using it. However, this intervention has not been used as anticipated. For example, some people in frontlines still reluctant to use it due to fearing punishment, while others have used it on occasions that it did not require to stop the operations. The use of this intervention in the offshore industry requires more guidance and training.
} 
complacency and dependence on one another, particularly when searching for errors and detecting and correcting anomalies on time. Model simulations show this: stronger social ties among employees result in a reduced preoccupation with failure (PWF). A remedy for this negative cultural tendency is to concurrently foster a sense of personal accountability, so that leadership and employees self-assess and self-correct their participation in maintaining safer operations. Accordingly, personal accountability resulted in high-performance levels in most of the five HROT principles, particularly PWF.

4. Pro-Social motivation and Emotional Ambivalence: Based on the responses of the industry's participants who took part in this study, some organizations in the offshore oil and gas industry are notably practicing these two interventions. While the expected outcomes from these two interventions are different because each one is designed to achieve specific purposes, they complement each other. As prosocial motivation builds confidence and trust around the corporate vision and the self, which increases the employee's level of commitment and participation, emotional ambivalence maintains caution and alertness that things can go wrong. The model simulation results based on each intervention individually agree with the industry practitioners' feedback because these practices lead to the comprehensive and relatively high-performance practice of HROs.

\subsection{Review of Research Questions and Results}

This section summarizes my findings related to the research questions that I posed in Section 2.8.4. 


\subsubsection{Addressing the lack of System Perspectives}

In Section 2.8.1, I identified the need to approach and represent HROT five principles from a system perspective. I stated two objectives, namely (1) to develop a novel, mixedmethods approach to modeling the principles of HROT as a Fuzzy Cognitive Map (FCM), based on published, peer-reviewed research, and (2) To develop a comprehensive FCM system model of HROT, using the novel approach. Achieving these objectives required answering a series of methodological questions relating to analyzing the literature and the FCM modeling process, which I will discuss one-by-one.

Research Question 1: How can research publications on HROT be analyzed to identify and represent relevant concepts and causal relationships?

I answered RQ 1 by first conducting a systematic literature review to identify relevant sources and then analyze them by combining thematic analysis and thematic network techniques - two separate but related qualitative inquiry methods. I chose these methods (and used Atlas. ti, a software for qualitative research) since my objective was to analyze the published literature on HROT for relevant concepts and causal relationships and build a network of these causally related concepts transparently and rigorously. My work demonstrates (for the first time, to my knowledge), that thematic analysis and thematic network technique are well suited as methodological foundations for FCM modeling.

Research Question 2: How can the weight of causal relationships be inferred from qualitative data in research publications? 
This question deals with a reoccurring problem in all FCM studies, namely the proper assignment of causal weights, which often occurs based on expert judgment. Because my work builds on published literature, this was not an option. Accordingly, I had to develop a novel way of inferring causal weights from qualitative data and using a measure called “t-coefficient," based on a recently published paper by Armborst (2017). This approach leverages my qualitative analysis and uses a statistical measure for thematic proximity, which provides information about the strength of causal connections between themes because I coded the research texts with a focus on causality. Because my method is novel regarding the approach and with regard to its application to FCM models, I had to ensure its validity. I achieved this by comparing results to other quantitative measures used to infer weights of causal relationships from qualitative data, as well as by carefully testing the resulting FCM model.

\section{Research Question 3: How can information about concepts, causal relationships, and relationship weights be used to develop a general FCM model of HROT?}

RQ3 was focused on applying the results for RQ 1 and 2 to create a generally applicable model of HROT and, in the process, develop a structured, mixed-method approach for creating and testing FCM models based on published literature. The result was an FCM model that represented the five HROT principles and the concepts that contribute to them, as well as the interdependencies between contributing concepts, principles, and the overall objective of organizational reliability. The developed FCM-HROT is a standardized model that does not represent a specific industry since it characterizes the phenomenon of 
organizational reliability (OR) in any system. Therefore, this model is like a core engine for any industry that aspires to contextualize and use it to improve its organizational reliability.

Testing and running simulations based on the model reveal several things about the model and - by extension - HROT theory, which is discussed in more depth in Section 5.1 and Section 6.1. Importantly, the model is policy sensitive, meaning that it can identify concepts (or combination of concepts) that impact the five HROT principles differently and lead to different levels of organizational reliability. Moreover, the model clearly shows the systemic nature of organizational reliability because concepts frequently impact multiple HROT principles through direct and indirect effects, and these impacts can lead to unintended consequences. In particular, as I had hypothesized when beginning this research, it is difficult to simultaneously improve all HROT principles because improvements in one of the principles can reduce other principles. Accordingly, a system model for understanding HRO dynamics is essential from a theoretical perspective, as well as a practical planning tool.

\section{Research Question 4: How can uncertainty about the appropriate model structure be} managed in the modeling process?

One of the challenges of modeling organizational reliability is the lack of directly measurable concepts and reliable data on real-world systems: companies simply do not track all the elements that are understood to contribute to organizational reliability. This is the main reason why HROT research strongly relies on qualitative research. Accordingly, there is uncertainty about the appropriate model structure that best reflects and replicates 
the real-world system's behavior, resulting in RQ 4 above. In my research, I have addressed this in two ways:

First, I subjected the model to a series of verification tests that I borrowed from the literature on system dynamics and adapted to my case. I examined the model structure and behavior to identify discrepancies between what the model shows and what behavior one would expect according to the literature. Although two cases (see Section 5.1.2) have shown abnormal behaviors that required thorough examination and calibration of the model structure, the model has passed all the tests during both the pilot and expanded studies. Second, I identified causal relationships that were uncertain because study participants assigned different weights and coped with this type of uncertainty by implementing the procedures of the EMA technique, which results in an ensemble of scenarios, each reflecting different model configurations and input vectors. I used these insights to identify managerial interventions that were robust in the sense that they delivered desirable and largely similar results, regardless of how the model structure varied in the EMA process. Managers can choose these robust strategies and be confident that they will deliver the desired results. I also identified situations in which uncertainty about the model structure did not permit a confident selection of the best intervention. This information alerts decision-makers to cases where they might want to invest more time and effort into data collection, closely monitor the outcomes of chose interventions to understand how they impact objectives and accompany interventions with additional actions to prevent possible undesired results. Thus, my work developed practical pathways for managing uncertainty 
about model structure in many FCM modeling projects that are applicable beyond this research scope.

\subsubsection{Addressing the Lack of Contextualization for Offshore Oil and Gas}

In Section 2.8.2, I identified the need to contextualize HROT principles for the specific conditions in offshore oil and gas and stated the objective to do so with the help of experts:

\section{Research Question 5: How can the FCM model of HROT practices be contextualized to the specific settings of the offshore oil and gas operations?}

This step is necessary so that the model reflects real-world behaviors and predicts the outcomes of interventions. Contextualizing the model requires expanding the core model of general HROT dynamics by adding an outer model layer that describes managerial interventions and how they relate to the core model's exogenous driver variables. I identified the interventions by reviewing the academic and practitioner literature and established their linkages to the core model with industry practitioners' help. The latter occurred in two phases: (1) a Pilot study, by which I collected the required data to contextualize the model from industry experts during an in-person workshop, and (2) an expanded study, where I collected a more comprehensive range of data via an online survey. In both studies, I provided a list of interventions and the corresponding lists of HRO-relevant concepts (i.e., exogenous variables in the core model). I asked them to provide their opinions on whether a specific intervention increases, decreases, or does not affect the concepts (to determine relationship directions and signs) and to what extent (to determine relationship weights). The result is a new model that reflects HROT principles 
and interventions, based on experts' knowledge of their industry. Simulating interventions in the model shows how they change the five HROT principles and contribute to safety culture.

\subsubsection{Addressing the Lack of Practical Planning Approaches}

In Section 2.8.3, I have identified the need for a comprehensive approach that is supporting HROs planning since the industry decision-makers lack support in selecting interventions that: (1) can be expected to improve organizational reliability, (2) are synergistic with other planned interventions to improve reliability, and (3) are robust under changing conditions. Thus, I stated my objective to evaluate the FCM model as a tool for planning HROs practices and achieve it. It requires answering the following research question, which I discuss before.

Research Question 6: How can the contextualized FCM model of HROT practices be used to support safety decision-makers in the offshore oil and gas industry?

I answered RQ 6 by introducing and implementing a structured process that constitutes planning interventions to leverage organizational reliability by enforcing positive cultural changes in the workforce's behaviors and attitudes. This process is completing the process of contextualizing the core model. In addition to the selection of individual or groups of interventions based on the outcomes that they are anticipated to make, this process also consists of simulating the model based on the selected interventions using traditional and EMA simulation techniques and then analyzing and interpreting the resulted performance 
of the five HROT principles. Through several simulations, the process has shown that the resulting performances have significantly reflected the relevance and the desirability of the applied intervention or group of interventions to the offshore oil and gas operations with respect to the opinions of the industry's safety decision-makers.

This process has shown that the contextualized model of HROT can support the safety decision-makers by focusing their efforts in planning and implementing various safety programs, interventions, strategies, and policies and selecting the plans that yield desired cultural changes and that are feasible and synergetic with the other plans that the organizations have already implemented. This is particularly essential since selecting the right plan often requires actual experiments (sometimes by trial and error) as well as the allocation of various resources, outstanding efforts, and long times. Moreover, the use of the EMA technique with FCM has, in fact, leveraged the implications of this tool in attaining ranges of outcomes instead of only one for each case, which expands the basis of the decision-making process and enables safety decision-makers to comprehensively deliberate and decide which plans they should consider and which ones they should not. 


\section{Research Limitations and Contributions}

This chapter discusses the limitations of this research and the theoretical, methodological, and practical contributions it has made. Section 8.1 addresses all the limitations that this research has encountered and coped with, while Section 8.2 states in detail all the contributions.

\subsection{Research Limitations}

This research has several limitations, including context data limitations (to contextualize the model) and the limitations inherent in the literature and the utilized method. These limitations are discussed in the following sections.

\subsubsection{Context Data Limitations}

Attaining data from the research context (i.e., the offshore oil and gas industry) to contextualize the model was difficult. Industry practitioners told me that some people working in this industry avoid participating in such studies because they fear repercussions for their jobs or companies, even if they are not identifiable in research reports (as was the case for the workshop) or if their identity is unknown to the researchers (as was the case in the survey). This is particularly true for operators on the offshore rigs, who are also difficult to reach. Accordingly, the people in my study are more involved in safety consultation and management on corporate levels as well as safety and health regulations, auditing, and compliance (e.g., safety manager, safety influencers, HSE, compliance managers, risk managers, operations remote controllers, etc.). While they are undoubtedly knowledgeable 
about safety interventions and effects, their perspectives are limited by their roles and the companies they work for.

Future studies may want to include more employees in different roles, including those on the front-lines and a more diverse set of companies, including smaller operations.

\subsubsection{Literature Limitations}

Despite the rich and broad literature on high reliability organizations theory HROT in describing how organizations can theoretically achieve highly reliable performance, not every aspect has been explicitly discussed, particularly based on a cause and effect reasoning. Therefore, interpreting the theoretical arguments concerning HRO-relevant concepts and how they cause one another might encounter certain subjectivity levels, especially that drawing an underlying path for these augments based on the causes and effects is critical in developing a causal-descriptive model. That is why I used a particular coding strategy and procedure and followed certain types of qualitative coding (causation coding) that put the entire process on the path of causality. This design has effectively reduced the level of subjectivity and retained research quality.

Moreover, the literature does not provide explicit information about which concepts contribute more versus less to organizational reliability. Still, some concepts are more extensively discussed and more deeply investigated in the literature than others. This is reflected in the coding process in two ways: (1) concept grounding, which means the number of times the concepts are mentioned in the selected documents, and (2) concept centrality in the FCM-HROT model that results from the coding process. 
Another literature limitation is that some factors, such as those I considered excluded from the core model boundaries (e.g., labor market - see Table 16), can potentially have significant effects on the performance of the five principles through one or more of the corresponding HRO-relevant concepts. However, the literature on HROT discusses these excluded factors only marginally. It focuses on the factors that I already extracted and included in the developed core model as exogenous and endogenous elements. This poses a question that worth future investigation to examine the relevance of some excluded factors to whether or not they should be considered exogenous or endogenous elements of the core model.

\subsubsection{Methodological Limitations}

This research uses the fuzzy cognitive map (FCM) as the main methodology, which has several inherent limitations that apply to this research. I have created the FCM model across two phases: Developing a cognitive map and assigning weights to each causal link in the map. In this research, the cognitive map has been developed by following a logical design and semi-automated techniques. Combining the used methods is, to my knowledge, new to FCM methodology and has yielded a relatively large cognitive map (51 concepts and 138 causal links), which makes assigning weights to this large number of connections using expert opinions is very difficult. Thematic proximity was used to overcome this issue, which is also new to FCM. Although it was tested and found to be a reliable combination, the use of these techniques is new and yet to be standardized.

Furthermore, FCM does not consider time lags in the computational process, which may not reflect the actual reality but otherwise predict it. For example, in real-world operations, 
implementing an intervention such as practicing emergencies can increase both awareness and experience of the workforce; however, while knowledge can be increased at the same time when the practice is held, experience takes years to be developed. Nevertheless, modeling these activities using FCM can yield increases in both awareness and experience. The literature has suggested using the so-called "dummy concepts" to break up the longterm causal link into several causal relationships (Jetter, 2006). But this approach can add more complications to the developed FCM because it increases the number of concepts and causal relationships, mainly when time lags present in the model.

Another limitation is that the simulation results of the FCM-HROT model can provide degrees of change in the performance of the five HROT principles rather than absolute values, which makes the interpretation of the results somewhat subjective.

\subsection{Research Contributions}

Based on the outcomes from this research, the following sections discuss the theoretical, methodological, and practical contributions.

\subsubsection{Theoretical Contribution}

This research has made the following theoretical contributions, perhaps for the first time in the literature on high reliability organizations theory (HROT).

1. Moved the Academic Research on High Reliability Theory (HROT) from its current state of a more qualitative and theoretical context to a more quantitative and practical space that can assess the potential capabilities regarding HRO-relevant practices in complex and high hazard systems. Despite more than thirty years of extensive academic 
research on HROT, the theory for various reasons has remained in the conceptual construct with few attempts to move it into practice. This dissertation has attempted to move the research on HROT to the real-world space by empirically testing organizational reliability based on expert-provided data and practically achieved its theoretical construct of the five principles (i.e., the package) in few cases, through which all the five principles have been influenced concurrently, such as the outcomes of simulating the model based on training interventions.

2. Developed a Comprehensive System Model Based HROT that, perhaps for the first time, empirically verifies why implementing the HROT five principles could lead to accomplishing reliable performance and also explains why HROT can achieve what alternative approaches could not achieve for complex systems. This model serves as a platform for testing theoretical assumptions regarding safety culture and safety-relevant practices and as a reference for expanding the boundaries of high reliability theory by testing new hypotheses.

3. Created a Reliable Assessment Technique by which organizations can assess their readiness or the level of the ongoing progress to becoming HROs. This technique enables organizations to quantitatively identify the extent to which they implement the HROT five cognitive processes with respect to their policies and strategies regarding safety-related practices. It can inform organizations whether a specific theoretical approach is attainable due to their practical and organizational settings.

\subsubsection{Methodological Contribution}

The following are the methodological contributions that this research has made. 
1. Developed a Novel Approach to Modeling Complex Systems by integrating various methods and techniques, including fuzzy cognitive maps FCMs, thematic analysis, and thematic network for the first time as an innovative, structured, tested, and fully documented process that represents a groundbreaking approach for modeling complex phenomenon based on purely published literature. This approach, in fact, has expanded the practice of FCM methodology to build models from peer-reviewed published research through the use of thematic analysis and thematic network for the first time by identifying latent patterns and causally related themes, and as a means to elicit the required qualitative knowledge for building cognitive maps.

2. First Time Self-Quantifying the edges of a Cognitive Map through the use of thematic proximity technique to convert the content of the published qualitative and textual data of the causally related arguments by the theorists and the academic experts into numerical values that represent the strength of these relationships. For the first time, this method alternates the use of subject experts in building and quantifying the edges of FCM models.

\section{Combined EMA with FCM to Overcome Uncertainties and Interpret Disagreements}

that often result from the variations and differences in the experts' opinions regarding the signs and the weights of causal relationships in the FCM models. Combining these methods allows simulating a vast number of the FCM model structures instead of only one, as in the traditional simulation of consolidative FCM models. Therefore, the simulation process yields a range of outcomes that represent various degrees of change in the performance of the concepts of interest. This enables a broader span for 
interpreting the outcomes of each case. Accordingly, disagreements can result in a wide range of tradeoffs, which provides safety decision-makers with more possibilities to make the best decisions and chose the plans that align better with the organizational and operational conditions of their organizations.

\subsubsection{Practical Contribution}

As this research has empirically tested and evaluated the developed FCM-HROT model with respect to the experts-provided data, the following summarizes its practical contributions.

1. Created a Practical Tool for informing safety decision-makers and guiding their decisions around safety-relevant approaches and practices. This tool will help their understanding of how to best achieve higher levels of safety. Practitioners can use this tool to test the feasibility and effectiveness of designing and implementing managerial interventions, safety programs, and initiatives with respect to their organizational strategies before allocating resources to implement these programs. They can also test their strategies and safety policies and scenarios by simulating the model to anticipate the system's current and future state based on these specific strategies.

2. Established a Platform for Training where the model (as a flight simulator) will help practitioners, particularly operators and frontline workers, experiment with simulated emergencies. These simulations test and enhance their commitment to resilience through learning how to anticipate, cope with, and learn from failures, as well as learning how to prevent and/or mitigate the consequences of large-scale accidents. 


\section{References}

Aase, K. \& Tjensvoll, T., 2003. Learning In High Reliability Organizations (HROs):

Trial Without Error. Barcelona, Spain, Warwick Business School.

Aboagye-Nimo, E., Raiden, A., King, A. \& Tietze, S., 2013. A safety culture shaped by common sense. Reading, UK, Association of Researchers in Construction Management.

Abraham, A., 2005. Artificial Neural Networks. In: P. H. Sydenham \& R. Thorn, eds. Handbook of Measuring System Design. Hoboken, NJ: John Wiley \& Sons, Ltd, pp. 901-908.

Acree, B., Hansen, E., Jansa, J. \& Shoub, K., 2016. Comparing and Evaluating Cosine Similarity Scores, Weighted Cosine Similarity Scores, \& Substring Matching. [Online]

Available at: http://hansen.web.unc.edu/files/2014/12/AHJS Weighted Cosine.pdf [Accessed 2018].

Agusdinata, D. B., 2008. Exploratory modeling and analysis:: a promising method to deal with deep uncertainty. Delft, Netherlands: Technology, Policy and Management, TU Delft.

Agwu, M., 2012. Impact of Employees Safety Culture on Organizational Performance in Shell Bonny Terminal Integrated Project (BTIP). European Journal of Business and Social Sciences, 1(5), pp. 70-82.

Alibage, A. et al., 2018 . Exploratory Participatory Modelling with FCM to Overcome Uncertainty: Improving Safety Culture in Oil and Gas Operations. Fort Collins, Colorado, Brigham Young University: https://scholarsarchive.byu.edu/iemssconference/2018/Stream-C/90/.

Alizadeh, Y., 2018. Achieving Organizational Ambidexterity: An Exploratory Model, Using Fuzzy Cognitive Maps. PhD Dissertation ed. Portland, OR: Department of Engineering \& Technology Management, Portland State University.

Alizadeh, Y. \& Jetter, A., 2017. Content Analysis Using Fuzzy Cognitive Map (FCM): A Guide to Capturing Causal Relationships from Secondary Sources of Data. Portland, 
OR, Portland International Conference on Management of Engineering and Technology.

Amalberti, R., 2013. Navigating Safety: Necessary Compromises and Trade-Offs -

Theory and Practice. New York : Springer Dordrecht Heidelberg.

Amer, M., Daim, T. U. \& Jetter, A., 2013. A review of scenario planning. Futures, Feb, 2013, Vol.46, p.23(18), Volume 46, pp. 23-40.

Aminpour, P., 2018. PyFCM: python for fuzzy cognitive mapping. s.1.:Github.com.

Anderson, C. L., 2010. IT is risky business: Three essays on ensuring reliability, security, and privacy in technology mediated settings.. College Park, MD: Doctoral Dissertation, University of Maryland.

Antonsen, S., 2009. Safety Culture: theory, Method and Improvement. Farnham: Ashgate Publishing Limited.

Armborst, A., 2017. Thematic Proximity in Content Analysis. SAGE , 7(2), p. 1-11. Attride-Stirling, J., 2001. Thematic networks: an analytic tool for qualitative research. Qualitative Research, 1(3), pp. 385-405.

Aven, T. \& Krohn, B. S., 2014. A New Perspective on How to Understand, Assess and Manage Risk and The Unforeseen. Reliability Engineering and System Safety, Volume 121, pp. 1-10.

Axelrod, R., 1976. Structure of Decision: The Cognitive Maps of Political Elites. Princeton, NJ: Princeton University Press.

B\&W Pantex, 2008. Hight Reliability Ooperations: A practical Guide to Avoid System Accident. Amarillo, TX: B\&W Pantex .

Baker, D. P., Day, R. \& Salas, E., 2006. Teamwork as an Essential Component of HighReliability Organizations. Health Services Research, 41(4), pp. 1576-1598.

Bankes, S., 1993. Exploratory Modeling for Policy Analysis. Operations Research, 41(3), pp. 435-449.

Bankes, S. C., 1992. Exploratory Modeling and the Use of Simulation for Policy Analysis, Santa Monica, CA: RAND Coporation.

Barlas, Y., 1996. Formal aspects of model validity and validation in system dynamics. System Dynamics Review, 12(3), pp. 183-210. 
Barrett, M. S., Novak, J. M., Venette, S. J. \& Shumate, M., 2006. Validating the high reliability organization perception scale. Communication Research Reports, 23(2), pp. 111-118.

Barton, M. A. \& Sutcliffe, K. M., 2009. Overcoming Dysfunctional Momentum:

Organizational Safety as A Social Achievement. Human Relations, 62(9), pp. 13271356.

Beatrice, O. O., 2011. Influencing safety culture in the UK Offshore Oil and Gas Industry: The importance of employee involvement. Aberdeen: OPITO - Skills for Oil and Gas.

Berardi, P. J., 2010. Measuring High Reliability Characteristics of the Organization, Emmitsburg, MD: National Fire Academy.

Bevilacqua, M., Ciarapica, F., Mazzuto, G. \& Paciarotti, C., 2013. Application of fuzzy cognitive maps to drug administration risk management. FAC Proceedings Volumes, 46(7), pp. 438-443.

Bigley, G. A. \& Roberts, K. H., 2001. The Incident Command System: High-Reliability Organizaing for Complex and Volatile Task Environment.(Statistical Data Included). Academy of Management Journal, 44(6), pp. 1281-1300 .

Black, A. E. \& McBride, B. B., 2013. Assessing high reliability practices in wildland fire management : an exploration and benchmarking of organizational culture, Fort Collins, CO: United States Department of Agriculture.

Bogue, B., 2009. How Principles of High Reliability Organizations Relate to Corrections. Federal Probation, Dec, 2009, Vol.73(3), p., 73(3), pp. 22-27.

Boin, A. \& Schulman, P., 2008. Assessing NASA's Safety Culture: The Limits and Possibilities of High-Reliability Theory. Public Administration Review, 68(6), pp. 1050-1062.

Borges, M. d. L. \& Gonçalo, C. R., 2010. Learning Process Promoted by Sensemaking And Trust: A Study Related to Unexpected Event. Cadernos EBAPE.BR, 8(2), pp. 260-277.

Borys, D., Else, D. \& Leggett, S., 2009. The fifth age of safety: the adaptive age. Journal of Health \& Safety Research \& Practice, 1(1), pp. 19-27. 
Boyatzis, R. E., 1998. Transforming Qualitative Information: Thematic Analysis and Code Development. 1st ed. Thousand Oaks, CA: SAGE Publications, Inc.

Bradley, E. H., Curry, L. A. \& Dever, K. J., 2007. Qualitative Data Analysis for Health Services Research: Developing Taxonomy, Themes, and Theory. Health Services Research, 42(4), p. 1758-1772.

Braun, V.\& Clarke, V., 2006. Using thematic analysis in psychology. Qualitative Research in Psychology, 3(2), pp. 77-101.

Braun, V.\& Clarke, V., 2014. What can thematic analysis offer health and wellbeing researchers?. International Journal of Qualitative Studies on Health and Well-being, 9(1).

Braun, V., Clarke, V. \& Weate, P., 2016. Using thematic analysis in sport and exercise research. In: B. Smith \& A. C. Sparkes, eds. Routledge Handbook of Qualitative Research in Sport and Exercise. London, UK : Routledge, pp. 191-205.

BSEE, 2013. BSEE Final Safety Culture Policy Statement, Washington, DC: The Bureau of Safety and Environmental.

BSEE, 2014. Annual Report, Washington, DC: The Bureau of Safety and Environmental Enforcement.

BSEE, 2015. Annual Report, Washington, DC: The Bureau of Safety and Environmental Enforcement.

BSEE, 2016. Annual Report, Washington, DC: The Bureau of Safety and Environmental Enforcement.

BSEE, 2017. Annual Report, Washington, DC: The Bureau of Safety and Environmental Enforcement.

BSEE, 2018. Offshore Incident Statistics, Washington, DC: Bureau of Safety and Environmental Enforcement.

BSEE, 2019. Annual Summary of Production for Entire Region-GULF OF MEXICO REGION, Washnigton DC: United State Department of the Interior - The Bureau of Safety and Environmental Enforcement .

Busby, J. \& Iszatt-White, M., 2014. The Relational Aspect to High Reliability Organization. Journal of Contingencies and Crisis Management, 22(2), pp. 69-80. 
Busby, J. S., 2006. Failure to Mobilize in Reliability-Seeking Organizations: Two Cases from the UK Railway. Journal of Management Studies, 43(6), pp. 1375-1393.

Carley, K. M., 1997. Extracting team mental models through textual analysis. Journal of Organizational Behavior, Volume 18, pp. 533-558.

Carlo, J. L., Lyytinen, K. \& Boland, R. J. ,. J., 2012. Dialectics of collective minding: contradictory appropriations of information technology in a high-risk project. MIS Quarterly, 36(4), pp. 1081-1108.

Carnino, A., 2000. Management of Safety, Safety Culture and Self Assessment. Bled, Slovenia, International Atomic Energy Agency.

Carvalho, J., Wise, L., Murta, A. \& Mesquita, M., 2008. Issues on Dynamic Cognitive Map Modelling of Purse-seine Fishing Skippers Behavior. Hong Kong, China, IEEE International Conference on Fuzzy Systems, pp. 1503-1510.

Casler, J., 2014. Revisiting nasa as a high reliability organization. Public Organization Review, 14(2), pp. 229-244.

Christian, M. S., Wallace, J. C., Bradley, J. C. \& Burke, M. J., 2009. Workplace Safety: A Meta-Analysis of the Roles of Person and Situation Factors. Journal of Applied Psychology, 94(5), pp. 1103-1128.

Christianson, M. K. \& Sutcliffe, K. M., 2008. Sensemaking, High-reliability Organizing, and Resilience. In: P. Croskerry, K. S. Cosby, S. M. Schenkel \& R. L. Wears, eds. In Patient Safety in Emergency Medicine. Philadelphia, PA: Lippincott Williams \& Wilkins, pp. 27-33.

Clarke, S., 1999. Perceptions of organizational safety: implications for the development of safety culture. Journal of Organizational Behavior, 20(2), pp. 185-198.

Clarke, V., 2017. Thematic analysis: What is it, when is it useful, and what does 'best practice' look like?, Auckland, New Zealand: The University of Auckland.

Clarke, V. \& Braun, V., 2014. Thematic Analysis. In: A. C. Michalos, ed. Encyclopedia of Quality of Life and Well-Being Research. Netherlands: Springer , pp. 6626-6628.

Cook, K. E., 2011. Reliability assessments in qualitative health promotion research. Health Promotion International, 27(1), pp. 90-101. 
Cooper, D., 2013. Strategic Safety Culture Roadmap. Franklin, IN: B-Safe Management Solution Inc. .

Cooper, M. D., 1998. Improving Safety Culture: A Practical Guide. Chichester: J. Wiley Cooren, F., 2004. The Communicative Achievement of Collective Minding: Analysis of Board Meeting Excerpts. Management Communication Quarterly, 17(4), pp. 517551.

Cox, S. \& Cheyne, A., 2000. Assessing Safety Culture in Offshore Environments. Safety Science, 34(1), pp. 111-129.

Cox, S. \& Cox, T., 1991. The Structure of Employee Attitudes to Safety. Work \& Stress, 5(2), pp. 93-106.

Cox, S., Jones, B. \& Collinson, D., 2006. Trust relations in high-reliability organizations. Risk Analysis, 25(5), pp. 1123-1138.

Davis, J. P., Eisenhardt, K. M. \& Bingham, C. B., 2007. Developing theory through simulation methods. The Academy of Management Review, 32(2), pp. 480-499.

Deal, T. E. \& Kennedy, A. A., 1982. Corporate Cultures: The Rites and Rituals of Corporate Life. Reading, MA: Addison-Wesley Publishing Company.

Dekker, S. W. \& Woods, D. D., 2010. The High Reliability Organization Perspective. In: E. Salas, F. Jentsch \& D. Maurino, eds. Human Factors in Aviation. Cambridge, MA: Academic Press, p. 123-143.

Dikopoulou, Z. \& Papageorgiou, E., 2017. Inference of Fuzzy Cognitive Maps (FCMs). s.l.:cran.r-project.org.

Edwards, J. R., Davey, J. \& Armstrong, K., 2013. Returning to the roots of culture: A review and re-conceptualisation of safety culture. Safety Science, Volume 55, pp. 7080.

Fleming, M., 2001. Safety culture maturity model, Norwich: Health and Safety Executive.

Fleming, M. \& Scott, N., 2012. Cultural Disasters: Learning From Yesterday's Failures To Be Safe Tomorrow. Oil and Gas Facilities Society of Petroleum Engineers. Houston, Texas, 1(3), pp. 1-8. 
Forrester, J. \& Senge, P., 1980. Tests for building confidence in system dynamics models. TIMS Studies in the Management Sciences, 12(1), p. 209 - 228.

Forrester, J. W., 2009. Some Basic Concepts in System Dynamics, Cambridge, MA: Sloan School of Management, Massachusetts Institute of Technology.

Frankel, A. S., Leonard, M. W. \& Denham, C. R., 2006. Fair and Just Culture, Team Behavior, and Leadership Engagement: The Tools to Achieve High Reliability. Health Services Research, 41(42), pp. 1690-170.

Friese, S., 2018. ATLAS.ti 8 Windows User Manual. Berlin.: ATLAS.ti Scientific Software Development GmbH.

Gebauer, A., 2013. Mindful Organizing as a Paradigm to Develop Managers. Journal of Management Education, 37(2), pp. 203-228.

Glendon, A. \& Stanton, N., 2000. Perspectives on safety culture. Safety Science , 34(1), pp. 193-214.

Goff, R. J., Wilday, J. \& Holroyd, J., 2015. Creeping Changes. Edinburgh, Institution of Chemical Engineers (IChemE).

Goldenhar, L. M., Brady, P. W., Sutcliffe, K. M. \& Muething, S. E., 2013. Huddling for High Reliability and Situation Awareness. BMJ Quality \& Safety, 22(11), pp. 899906.

Götvall, J., 2014. A Framework for Assessing Safety Culture: Master's thesis in Chemical Engineering. Gothenburg, Sweden: Chalmers University of Technology.

Grabowski, M. \& Roberts, K. H., 2016. Reliability seeking virtual organizations: Challenges for high reliability organizations and resilience engineering. Safety Science.

Gray, S., Chan, A., Clark, D. \& Jordan, R., 2012 . Modeling the integration of stakeholder knowledge in social-ecological decision-making: Benefits and limitations to knowledge diversity. Ecological Modelling, Volume 229, pp. 88-96.

Gray, S., Zanre, E. \& Gray, S., 2014. Fuzzy Cognitive Maps as Representations of Mental Models and Group Beliefs. In: I. Elpiniki, ed. Fuzzy Cognitive Maps for Applied Sciences and Engineering. Heidelberg: Springer, p. 29-48. 
Gregor, S. \& Hevner, A. R., 2013. Positioning and presenting design science research for maximum impact.(Essay). MIS Quarterly, 37(21), pp. .337-355.

Gross, C. B., 2013. Spontaneous Images in the Mind: A Thematic Analysis of Psychoanalytic Literature on Psychotherapists' Unbidden Visualizations. Auckland, New Zealand: School of Public Health and Psychosocial Studies, Auckland University of Technology.

Guest, G., MacQueen, K. M. \& Namey, E. E., 2014. Applied Thematic Analysis. Thousand Oaks, CA: SAGE Publications, Inc.

Guldenmund, F., 2000. The nature of safety culture: a review of theory and research. Safety Science, Volume 34, pp. 215-257.

Guldenmund, F., 2010. Understanding and Exploring Safety Culture. Oisterwijk: Uitgeverij Boxpress.

Hajek, P., Prochazka, O. \& Pachura, P., 2017. Fuzzy cognitive maps based on text analysis for supporting strategic planning. Journal of Theoretical and Applied Information Technology, 95(23), pp. 6536-6545.

Hale, A. R. \& Hovden, J., 1998. Management and culture: the third age of safety. A review of approaches to organizational aspects of safety, health and environment. In: Occupational injury: Risk prevention and intervention. London: Taylor and Francis, pp. 129-167.

Hales, D. N. \& Chakravorty, S. S., 2016. Creating High Reliability Organizations Using Mindfulness. Journal of Business Research, 69(8), pp. 2873-2881.

Hales, D. N., Kroes, J., Chen, Y. \& Kang, K. W. (., 2012. The cost of mindfulness: A case study. Journal of Business Research, 56(4), pp. 570-578.

Harvey, E. J., Waterson, P. \& Dainty, A. R., 2016 . Applying HRO and resilience engineering to construction: Barriers and opportunities. Safety Science, pp. 1-11.

Hesamamiri, R., Mazdeh, M. \& Jafari, M., 2013. Measuring the reliability of knowledge management Instrument development and validation. Aslib Proceedings, 65(5), pp. 484-502. 
Hines, S. et al., 2008. Becoming a High Reliability Organization: Operational Advice for Hospital Leaders, Rockville, MD: Agency for Healthcare Research and Quality U.S. Department of Health and Human Services.

Hodges, J. S. \& Dewar, J. A., 1992. Is it You or Your Model Talking? A Framework for Model, Santa Monica, CA: RAND Coporation.

Hofstede, G., 1986. Cultural differences in teaching and learning. International Journal of Intercultural Relations, 10(3), pp. 301-320.

Hollnage, E., 2014. Safety-I and safety-II : the past and future of safety management. Farnham, England : Ashgate Publishing Limited.

Hopkins, A., 1999. The limits of normal accident theory. Safety Science, 1999 Jul-Aug, Vol.32(2-3), pp, 32(2-3), pp. 93-102.

Hopkins, A., 2006. Studying organisational cultures and their effects on safety. Safety Science, 40(10), p. 875-889.

Hopkins, A., 2007a. The Problem of Defining High Reliability Organisations, Canberra, Australia: National Research Center for OHS Regulation - The Australian National University.

Hopkins, A., 2007b. Lessons from Gretley: Mindful Leadership and the Law. 1 ed. Sydney: CCH Australia.

Høyland, S. A., Skotnes, R. Ø. \& Holte, K. A., 2018. An empirical exploration of the presence of HRO safety principles across the health care sector and construction industry in Norway. Safety Science, August 2018, Vol.107, pp., Volume 107, pp. 161172.

Hoy, W. K., Gage, C. Q. I. \& Tarter, C. J., 2006. School Mindfulness and Faculty Trust: Necessary Conditions for Each Other?. Educational Administration Quarterly, 42(2), pp. 236-255.

Hudson, P., 2007. Implementing a safety culture in a major multi-national. Safety Science, 45(6), pp. 697-722.

IAEA, 2002. Safety culture in nuclear installations: Guidance for use in the enhancement of safety culture, Vienna, Austria: International Atomic Energy Agency. 
ICAO, 2013. Safety Management Manual (SMM), Montréal, Quebec, Canada: International Civil Aviation Organization.

INSAG, 1991. Safety Culture, Viena: The International Atomic Energy Agency. Ismail, N., Kinchin, G. \& Edwards, J.-A., 2018. Pilot Study Does It Really Matter? Learning Lessons from Conducting a Pilot Study for a Qualitative PhD Thesis. International Journal of Social Science Research , 6(1), pp. 1-17.

Jahn, J. L. S., 2016. Adapting Safety Rules in a High Reliability Context: How Wildland Firefighting Workgroups Ventriloquize Safety Rules to Understand Hazards. Management Communication Quarterly, 30(3), p. 362-389.

Jahn, J. L. S. \& Black, A. E., 2017. A Model of Communicative and Hierarchical Foundations of High Reliability Organizing in Wildland Firefighting Teams. Management Communication Quarterly, 31(3), pp. 356-379.

Jette, A., Gray, S. \& Papageorgiou, E., 2018. Using Fuzzy Cognitive Map Modeling to promote system learning, public participation, and problem solving in socioenvironmental systems. Waikola, HI, Hawaii International Conference on System Sciences (HICSS-51).

Jetter, A. J., 2006. Fuzzy Cognitive Maps for Engineering and Technology Management: What Works in Practice?. Istanbul, Turkey, s.n.

Jetter, A. J. \& Kok, K., 2014. Fuzzy Cognitive Maps for futures studies-A methodological assessment of concepts and methods. Futures, Volume 61, pp. 45-57.

Jetter, A. J. \& Sperry, R. C., 2013 . Fuzzy Cognitive Maps for Product Planning: Using Stakeholder Knowledge toAchieve Corporate Responsibility. Maui, HI USA, s.n.

Jones, L. K. \& O’Connor, S. J., 2016. The Use of Red Rules in Patient Safety Culture. Universal Journal of Management, 4(3), pp. 130-139.

Kahn, H. \& Wiener, A. J., 1967. The Year 2000: A Framework for Speculation on the Next Thirty-Three Years. 1 ed. New York: MacMillan Publishing Company.

Kangari, R. \& Boyer, L. T., 1989. Basic concepts of the theory of fuzzy sets. Project Management Journal, 20(1), p. 44-46. 
Klein, K. J. et al., 2006. Dynamic Delegation: Shared, Hierarchical, and Deindividualized Leadership in Extreme Action Teams. Administrative Science Quarterly, 51(4), pp. 590-621.

Klein, R. L., Bigley, G. A. \& Roberts, K. H., 1995. Organizational Culture in High Reliability Organizations: An Extension. Human Relations, 48(7), pp. 771-793.

Kosko, B., 1986. Fuzzy cognitive maps. International Journal of Man-Machine Studies, 24(1), p. $65-75$.

Kosko, B., 1988. Hidden patterns in combined and adaptive knowledge networks. International Journal of Approximate Reasoning, 2(4), pp. 377-393.

Koso, B., 1991. Neural Networks and Fuzzy Systems A Dynamical Systems Approach to Machine Intelligence. 1st ed. Upper Saddle River, NJ: Prentice Hall.

Krippendorff, K., 2004. Content Analysis: An Introduction to Its Methodology. 1st ed. London, UK: Sage Publications Ltd.

Kwakkel, J. H. \& Pruyt, E., 2013. Exploratory Modeling and Analysis, an approach for model-based foresight under deep uncertainty. echnological Forecasting \& Social Change, 80(3), pp. 419-431.

Kwakkel, J., Walker, W. \& Marchau, V., 2010. From Predictive Modeling to Exploratory Modeling: How to use Non- Predictive Models for Decisionmaking under Deep Uncertainty. Coimbra, Portugal, 25th Mini-EURO Conference on Uncertainty and Robustness in Planning and Decision Making (URPDM 2010).

La Porte, T. \& Consolini, P., 1998. Theoretical and Operational Challenges of "HighReliability Organizations": Air-Traffic Control and Aircraft Carriers. International Journal of Public Administration, 21(6-8), pp. 847-852.

La Porte, T. R., 1996. High Reliability Organizations: Unlikely, Demanding and At Risk. Journal of Contingencies and Crisis Management, 42(2), pp. 60-71.

LaPorte, T. R. \& Consolini, P. M., 1991. Working in Practice but Not in Theory: Theoretical Challenges of High-Reliability Organizations. Public Administration Research and Theory, 1(1), pp. 19-48. 
LaPorte, T. R., Roberts, K. \& Rochlin, G. I., 1988. Aircraft Carrier Operations At Sea:The Challenges of High Reliability Performance, Berkeley, CA: University of California, Berkeley.

Lekka, C., 2011. High Reliability Organizations: A Review of The Literature, Harpur Hill, UK: Health and Safety Laboratory - HSE.

Lekka, C. \& Sugden, C., 2011. The Successes and Challenges of Implementing High Reliability Principles: A Case Study Of A UK Oil Refinery. Process Safety and Environmental Protection, 89(6), pp. 443-451.

Leveson, N., Dulac, N., Marais, K. \& Carroll, J., 2009. Moving Beyond Normal Accidents and High Reliability Organizations: A Systems Approach to Safety in Complex Systems. Organization Studies, 30(2-3), pp. 227-249.

Mackey, A. \& Gass, S. M., 2005. Second Language Research : Methodology and Design. 1st ed. London, UK: Routledge.

Madsen, P., Desai, V., Roberts, K. \& Wong, D., 2006. Mitigating Hazards Through Continuing Design: The Birth and Evolution of a Pediatric Intensive Care Unit. Organization Science, 17(2), pp. 239-248.

Marais, K., Dulac, N. \& Leveson, N., 2004. Beyond Normal Accidents and High Reliability Organizations: The Need for an Alternative Approach to Safety in Complex Systems. Cambridge, MA, Semantic Scholar.

Martınez-Corcoles, M., 2017. High Reliability Leadership: A Conceptual Framework. Journal of Contingencies and Crisis Management, pp. 1-10.

Martin, J., 2001. Organizational Culture: Mapping the Terrain. Thousand Oaks, CA: SAGE Publications, Inc.

Max, M. D., Johnson, A. H. \& Dillon, W. P., 2009. Economic Geology of Natural Gas Hydrate. Dordrecht: Springer.

McDonald, N., Schoenebeck, S. \& Forte, A., 2019. Reliability and Inter-rater Reliability in Qualitative Research: Norms and Guidelines for CSCW and HCI Practice. New York, NY, Association for Computing Machinery.

Mellor, N., Wilday, J., Lunt, J. \& Holroyd, J., 2015. High Reliability Organizations and Mindful Leadership. Edinburgh, Institution of Chemical Engineers (IChemE). 
Mitropoulos, P. \& Cupido, G., 2009. Safety as an emergent property: investigation into the work practices of high-reliability framing crews. Journal of Construction Engineering and Management, 135(5), pp. 407-516.

Moher, D., Liberati, A., Tetzlaff, J. \& Altman, D. G., 2009. Preferred reporting items for systematic reviews and meta-analyses: the PRISMA statement. Journal of Clinical Epidemiology, 2009, 62(10), pp. 1006-1012.

Morse, J. M. et al., 2002. Verification Strategies for Establishing Reliability and Validity in Qualitative Research. International Journal of Qualitative Methods, 1(2), pp. 1322.

Morton, Q., 2016. History of Oil: Beyond Sight of Land, London: GeoExPro.

Muhren, W. J., Van Den Eede, G. \& de Walle, B. V., 2007. Organizational Learning for the Incident Management Process: Lessons from High Reliability Organizations. Gallen, Switzerland, AIS eLibrary.

Nadkarni, S. \& Shenoy, P. P., 2004. A causal mapping approach to constructing Bayesian networks. Decision Support Systems, 38(2), pp. 259-281.

Nápoles, G., Papageorgiou, E., Bello, R. \& Vanhoof, K., 2016. On the convergence of sigmoid Fuzzy Cognitive Maps. Information Sciences, Volume 349-350, pp. 154-171. National Academies , 2016. Strengthening the Safety Culture of the Offshore Oil and Gas Industry, Washington, D.C.: The National Academies of Sciences, Engineering and Medicine.

National Commission, 2011. Deep Water: The Gulf Oil Disaster and the Future of Offshore Drilling, Washington D.C.: Report to the President. United States Goverment Printig Office.

National Energy Board, 2014. Advancing Safety In the Oil and Gas Industry: Statment On Safety Culture, Calgary, Alberta: National Energy Board (Canada).

Nyaupane, G. \& Buzinde, C., 2017. Scenario Planning: A Planning Tool for an Uncertain Future. Québec, Canada, 2017 ttra International Conference (Travel and Tourism Research Association). 
Ogliastri, E. \& Zúñiga, R., 2016. An introduction to mindfulness and sensemaking by highly reliable organizations in Latin America. Journal of Business Research, 69(10), pp. 4429-4434.

Oleinik, A., 2011. Mixing quantitative and qualitative content analysis: triangulation at work. Quality \& Quantity, 45(4), pp. 859-873.

Olsen, E., 2009. Safety climate and safety culture in health care and the petroleum industry: Psychometric quality, longitudinal change, and structural models.

Stavanger: University of Stavanger.

Özesmi, U. \& Özesmi, S. L., 2004. Ecological models based on people’s knowledge: a multi-step fuzzy cognitive mapping approach. Ecological Modelling, 176(1), pp. 4364.

Papageorgiou, E. I. \& Salmeron, J. L., 2013. A Review of Fuzzy Cognitive Maps Research During the Last Decade. IEEE Transactions on Fuzzy Systems, 21(1), pp. 66-79.

Papageorgiou, E. I. \& Salmeron, J. L., 2014. Methods and Algorithms for Fuzzy Cognitive Map-based Modeling. In: E. I. Papageorgiou, ed. Fuzzy Cognitive Maps for Applied Sciences and Engineering: From Fundamentals to Extensions and Learning Algorithms. Berlin: Springer, pp. 1-29.

Papageorgiou, G., Hadjis, A. \& Abrosimova, K., 2008. Management Flight Simulators; a New Approach to the Development of Decision Support Systems. WSEAS Transactions on Systems, 7(5), pp. 415-424.

Paranhos, E., Kozak, T. G. \& Boyd, W., 2017. Highly Reliable Organizations in the Onshore Natural Gas Sector: An Assessment of Current Practices, Regulatory Frameworks, and Select Case Studies, Golden, CO: National Renewable Energy Laboratory (NREL).

Parker, D., Lawrie, M. \& Hudson, P., 2006. A framework for understanding the development of organisational safety culture. Safety Science, 44(6), pp. 551-562.

PB National Commission , 2011. Deep Water: The Gulf Oil Disaster and the Future of Offshore Drilling, Washington D.C.: Report to the President. United States Goverment Printig Office. 
Perrow, C., 1984. Normal accidents : living with high-risk technologies. New York: Basic Books.

Pettersen, K. A. \& Schulman, P. R., 2016. Drift, Adaptation, Resilience and Reliability: Toward An Empirical Clarification. Safety Science.

Philp, J., 2003. Constraints on "noticingthe gap": Nonnative speakers' noticing of recasts in NS-NNS interaction. Studies in Second Language Acquisition, 25(1), pp. 99-126.

Pidgeon, N., 2010. Systems thinking, culture of reliability and safety. Civil Engineering and Environmental Systems, 27(3), pp. 211-217.

Pidgeon, N. \& O'Leary, M., 2000. Man-made disasters: why technology and organizations (sometimes) fail. Safety Science, 34(1), pp. 15-30.

Piers, M., Montijn, C. \& Balk, A., 2009. Safety culture framework for the ECAST SMS$W G$, s.1.: European Strategic Safety Initiative (ESSI).

Pourreza, P. et al., 2018. Health, Safety, Environment and Ergonomic Improvement in Energy Sector Using an Integrated Fuzzy Cognitive Map-Bayesian Network Model. International Journal of Fuzzy Systems, 20(4), pp. 1346-1356.

Priest, T., 2008. Technology and Strategy of Petroleum Exploration in Costal and Offshore Gulf of Mexico. In: History of the Offshore Oil and Gas Industry in Southern Louisiana. New Orleans(Louisiana): U.S. Department of the Interior, Minerals Management Service, Gulf of Mexico OCS Region, pp. 11-32.

Priest, T., 2008. Wake-Up Call: Accidents and Safty Provision in the Gulf of Mexico Offshore Industry. In: History of the Offshore Oil and Gas Industry in Southern Louisiana. New Orleans(Louisiana): U.S. Department of the Interior, Minerals Management Service, Gulf of Mexico OCS Region, p. 139-155.

Ray, J. L., Baker, L. T. \& Plowman, D. A., 2011. Organizational Mindfulness in Business Schools. Academy of Management Learning \& Education, 10(2), p. 188203.

Reason, J., 1997. Managing the Risks of Organisational Accidents. aldershot: Ashgate Publishing Limited.

Reason, J., 1998. Achieving a safe culture: theory and practice. Work and Stress, 12(3), pp. 293-306. 
Reason, J., 2000. Safety Paradoxes and Safety Culture. Injury Control \& Safety Promotion, 7(1), pp. 3-14.

Rerup, C., 2009. Attentional Triangulation: Learning from Unexpected Rare Crises. Organization Science, 20(5), pp. 876-893.

Richter, A., 2003. New ways of managing prevention-A cultural and participative approach. Safety Scince Monitor , 7(1).

Riffe, D., Lacy, S. \& Fico, F., 2014. Analyzing Media Messages Using Quantitative Content Analysis in Research. 3rd ed. NYC, NY: Routledge.

Roberts, K. H., 1990a. Managing high reliability organizations. California Management Review, 32(4), p. 101-114.

Roberts, K. H., 1990b. Some characteristics of one type of high reliability organization. Organization Science, 1(2), pp. 160-176.

Roberts, K. H., 2003. HRO Has Prominent History. Anesthesia Patient Safety Foundation Newsletter, 18(1).

Roberts, K. H. \& Libuser, C., 1993. From bhopal to banking: Organizational design can mitigate risk. Organizational Dynamics, 21(4), pp. 15-26.

Roberts, K. H. \& Martelli, P. F., 2016. A Variegated Approach to Occupational Safety. In: S. Clarke, R. J. Burke \& C. L. Cooper, eds. Occupational Health and Safety . New York : Routledge, pp. 325-343.

Roberts, K. H. \& Rousseau, D. M., 1989. Research in nearly failure-free, high-reliability organizations: having the bubble. IEEE Transactions on Engineering Management, 36(2), pp. 132-139.

Roberts, K. H., Rousseau, D. M. \& La Porte, T. R., 1994. The culture of high reliability: quantitative and qualitative assessment aboard nuclear-powered aircraft carriers. Journal of High Technology Management Research, 5(1), pp. 141-161.

Robson, L. S. et al., 2007. The effectiveness of occupational health and safety management system interventions: A systematic review. Safety Science, 45(3), pp. 329-353. 
Rochlin, G. I., 1993. Defining "high reliability" organisations in practice: a taxonomic prologue. In: K. H. Roberts, ed. New Challenges to Understanding Organisations. New York: Macmillan Coll Div, pp. 11-32.

Rochlin, G. I., 1996. Reliable Organizations: Present Research and Future Directions. Journal of Contingencies and Crisis Management, 4(2), pp. 55-60.

Rochlin, G. I., La Porte, T. R. \& Roberts, K. H., 1987. The Self-Designing HighReliability Organization: Aircraft Carrier Flight Operations at Sea. Naval War College Review, XL(4), pp. 76-90.

Rosness, R., Haavik, T. \& Tinmannsvik, R. K., 2016. Learning from What Do You Do When You Build Safety? Practitioners' Guide to Learning from Successful Operations, Trondheim, Norway: SINTEF.

Roth, E. M., Multer, J. \& Raslear, T., 2006. Shared situation awareness as a contributor to high reliability performance. Organization Studies, 27(7), pp. 967-988 .

Sagan, S. D., 1995. The Limits of Safety: Organizations, Accidents and Nuclear

Weapons. 2 ed. Princeton, NJ: Princeton University Press.

Saldaňa, J., 2016. The Coding Manaual for Qualitative Rsearchers. 3rd ed. Thousand Oaks, CA: SAGE Publications Inc.

Salleh, I., Ali, N., Yusof, K. \& Jamaluddin, H., 2017. Analysing Qualitative Data Systematically using Thematic Analysis for Deodoriser Troubleshooting in Palm Oil Refining. Chemical Engineering Transactions, Volume 56, pp. 1315-1320.

Salton, G., 1983. Introduction to Modern Information Retrieval. NewYork: McGrawHill Book Co..

Samuels, G. J., 2010. The Application of High-Reliability Theory to Promote Pain Management. The Journal of Nursing Administration , 40(11), pp. 471-476.

Sandelowski, M. \& Leeman, J., 2012. Writing Usable Qualitative Health Research Findings. Qualitative Health Research, 22(10), pp. 1404-1413.

Saunders, F. C., 2015. Toward High Reliability Project Organizing in Safety-Critical Projects. Project Management Journal, 46(3), pp. 25-35.

Saunders, M. N., Lewis, P. \& Thornhill, A., 2015. Research Methods for Business Students. 7th ed. London, UK: Pearson. 
Schein, E. H., 2004. Organizational Culture and Leadership. 3rd ed. San Francisco, CA: Jossey-Bass.

Schein, E. H., 2010. Organizational Culture and Leadership. 4th ed. San Francisco, CA: Jossey-Bass.

Schöbel, M., 2009. Trust in high-reliability organizations. Social Science Information, 48(2), pp. 315-333.

Shrivastava, P., 1987. Bhopal: Anatomy of Crisis. 1 ed. Cambridge, Mass: Ballinger Pub. Co..

Silbey, S. S., 2009. Taming Prometheus: Talk About Safety and Culture. The Annual Review of Sociology, Volume 35, p. 341-369.

Spencer-Oatey, H., 2000. Culturally Speaking: Managing Rapport Through Talk Across Cultures. New York, NY: Continuum.

Sterman, J. D., 2000. Truth and Beauty: Validation and Model Testing . In: Business Dynamics: Systems Thinking and Modeling for Complex World. NYC, NY: The McGraw-Hill Companies, pp. 845-891.

Sterman, J. D., 2002. All models are wrong: reflections on becoming a systems scientist. System Dynamics Review, 18(4), p. 501-531.

Sutcliffe, K. M., 2011. High Reliability Organizations (HROs). Best Practice and Research: Clinical Anaesthesiology, 25(2), pp. 133-144.

Sutcliffe, K. M., Paine, L. \& Pronovost, P. J., 2017. Re-Examining High Reliability: Actively Organizing for Safety. BMJ Quality \& Safety, 26(3), pp. 248-251.

Sutcliffe, K. M., Vogus, T. J. \& Dane, E., 2016. Mindfulness in Organizations: A CrossLevel Review. Annual Review of Organizational Psychology and Organizational Behavior, 3(1), pp. 55-81.

The Health Foundation, 2011. Evidence Scan: Measuring Safety Culture, London, Uk: The Health Foundation,.

Turner, B., 1995. Safety Culture Management: Safety Culture and its Context. Vienna, Austria, International Topical Meeting on Safety Culture in Nuclear Installations, American Nuclear Society, pp. 321-331. 
Turner, B. A., 1978. Man-Made Disasters. London : Wykeham Publications (London) Ltd. .

Turner, B. A., Pidgeon, N., Blockley, D. \& Toft, B., 1989. Safety Culture: Its Importance in Future Risk Management. Karlstad, Sweden, s.n.

Turner, B. A. \& Pidgeon, N. F., 1997. Man-made disasters. 2 ed. Boston, MA: Butterworth-Heinemann.

U.S.NRC, 2011. The Safety Culture Policy Statement, Rockville, MD: U.S. Nuclear Regulatory Commission.

Uttal, B., 1983. The Corporate Culture Vultures. Fortune, 108(8), pp. 66-72.

Valentine, S., Godkin, L. \& Varca, P., 2010. Role Conflict, Mindfulness, and Organizational Ethics in an Education-Based Healthcare Institution. Journal of Business Ethics, Volume 94, p. 455-469.

Valorinta, M., 2009. Information technology and mindfulness in organizations. Industrial and Corporate Change, 18(5), pp. 963-997.

van Vliet, M., Kok, K. \& Veldkamp, T., 2010. Linking stakeholders and modellers in scenario studies: The use of Fuzzy Cognitive Maps as a communication and learning tool. Futures, 42(1), pp. 1-14.

Vaughan, D., 1996. The Challenger Launch Decision: Risky Technology, Culture and Deviance at NASA. 1 ed. Chicago: University of Chicago Press.

Vogus, J. T. \& Sutcliffe, M. K., 2007a. The Safety Organizing Scale: Development and Validation of a Behavioral Measure of Safety Culture in Hospital Nursing Units. Medical Care, 45(1), pp. 46-54.

Vogus, J. T. \& Sutcliffe, M. K., 2007b. The Impact of Safety Organizing, Trusted Leadership, and Care Pathways on Reported Medication Errors in Hospital Nursing Units. Medical Care, 45(10), pp. 997-1002.

Vogus, T. J. \& Hilligoss, B., 2016. The Underappreciated Role of Habit in Highly Reliable Healthcare. BMJ Quality \& Safety, 25(3), pp. 141-146.

Vogus, T. J., Rothman, N. B., Sutcliffe, K. M. \& Weick, K. E., 2014. The Affective Foundations of High-Reliability Organizing. Journal of Organizational Behavior, 35(4), pp. 592-596. 
Vogus, T. J. \& Sutcliffe, K. M., 2012. Organizational Mindfulness and Mindful Organizing: A Reconciliation and Path Forward. Academy of Management Learning \& Education, 11(4), p. 722-735.

Vogus, T. J., Sutcliffe, K. M. \& Weick, K. E., 2010. Doing No Harm: Enabling, Enacting, and Elaborating a Culture of Safety in Health Care. Academy of Management Perspectives, 24(4), pp. 60-77.

Vogus, T. J. \& Welbourne, T. M., 2003. Structuring for high reliability: HR practices and mindful processes in reliability-seeking organizations. Journal of Organizational Behavior, Volume 24, pp. 877-903.

Vu, T. \& De Cieri, H., 2014. Safety culture and safety climate definitions suitable for a regulator A systematic literature review, Caulfield East: Australian Centre for Research in Employment and Work, Monash University.

Weick, K. E., 1987. Organizational culture as a source of high reliability. California Management Review , 29(2), pp. 112-127.

Weick, K. E., 2011. Organizing for Transient Reliability: The Production of Dynamic Non-Events. Journal of Contingencies and Crisis Management, 19(1), pp. 21-27.

Weick, K. E. \& Roberts, K. H., 1993. Collective mind in organizations: heedful interrelating on flight decks. Administrative Science Quarterly, 38(3), pp. 357-381.

Weick, K. E. \& Sutcliffe, K. M., 2001. Managing the Unexpected: Assuring High Performance in an Age of Complexity. 1 ed. San Francisco, CA: Jossey-Bass.

Weick, K. E. \& Sutcliffe, K. M., 2015. Managing the Unexpected: Sustained Performance in a Complex World. 3 ed. San Francisco, CA: Jossey-Bass.

Weick, K. E., Sutcliffe, K. M. \& Obstfeld, D., 1999. Organizing for High Reliability: Processes of Collective Mindfulness. Research in Organizational Behavior, Volume 1 (Stanford: Jai Press), p. 81-123.

Westrum, R., 1993. Cultures with Requisite Imagination. In: J. A. Wise, V. Hopkin \& P. Stager, eds. Verification and Validation of Complex Systems: Human Factors Issues. Berlin: Springer-Verlag, pp. 401-416.

Westrum, R., 2004. A typology of organisational cultures. Quality \& safety in health care, 13(Suppl 2), pp. 22-27. 
Wright, C., 1986. Routine deaths: fatal accidents in the oil industry. Sociological Review, 34(2), pp. 265-289.

Yin, R. K., 2017. Case Study Research and Applications: Design and Methods. 6 ed. Thousand Oaks, CA: SAGE Publications, Inc.

Youngberg, B. J., 2004. Assessing Your Organization's Potential to Become a High Reliability Organization. Journal of Healthcare Risk Management, 24(3), pp. 13-20.

Zadeh, L. A., 1965. Fuzzy sets. Information and Control, 8(3), pp. 338-353.

Zimmerman, E. H., 2012. What is Risk in Offshore Oil and Gas. Sea Technologies , 53(4), pp. 7-7.

Zimmerman, H.-J., 2001. Fuzzy Set Theory—and Its Applications. 4th ed. Dordrecht ; Boston: Kluwer Academic Publishers. 


\section{Appendix A: Empirical Studies on HROs}

Table 22: Empirical Research Studies on Organizational Reliability and Mindfulness

\begin{tabular}{|c|c|c|c|}
\hline Authors, Year & Research Context & Research Method & Research Outcomes \\
\hline (Hales, et al., 2012) & Health Care & Action Research & $\begin{array}{l}\text { This study shows that the five mindful principles can be counterproductive at } \\
\text { the beginning of implementation, but they become part of daily activities to } \\
\text { improve reliability. The research shows an improvement in the number of } \\
\text { adverse incidents between a nurse and the patient's family. }\end{array}$ \\
\hline (Carlo, et al., 2012) & $\begin{array}{l}\text { Architecture } \\
\text { Construction }\end{array}$ & Case Study & $\begin{array}{l}\text { The analysis reveals that collective mindfulness emerges from struggling with } \\
\text { inconsistencies in the five mindful elements. Implications for further study of } \\
\text { collective mindfulness and the assumption of IT capabilities were discussed as } \\
\text { well. }\end{array}$ \\
\hline (Bigley \& Roberts, 2001) & Fire Department & Case Study & $\begin{array}{l}\text { The study reveals that there is a possibility of new, highly bureaucratic, and } \\
\text { temporary organizational forms that can reach reliability under a broad range } \\
\text { of working conditions with high uncertainty and instability. }\end{array}$ \\
\hline (Madsen, et al., 2006) & Health Care & Case Study & $\begin{array}{l}\text { The study shows that the design of Pediatric Intensive Care Units was a } \\
\text { continuing effort, and its most stable component was a vision of distributed } \\
\text { knowledge and decentralized intensive care. }\end{array}$ \\
\hline (Rerup, 2009) & $\begin{array}{l}\text { Pharmaceutical } \\
\text { Industry }\end{array}$ & Case Study & $\begin{array}{l}\text { The link between the three studied dimensions (stability, vividness, and } \\
\text { coherence) suggest that attention is not just a structural phenomenon that can } \\
\text { be enacted and controlled by top management, but rather, it is a discrete and } \\
\text { evolving process that can help to understand the dynamism of how } \\
\text { organizations detect weak cues and learn from rare events when examined } \\
\text { carefully. }\end{array}$ \\
\hline (Valorinta, 2009) & Retail Organizations & Case Study & $\begin{array}{l}\text { The study concluded that information technology decreases mindfulness and } \\
\text { obstruct organizational adoption by promoting cognitive inertia and making the } \\
\text { enactment of change more challenging. }\end{array}$ \\
\hline
\end{tabular}




\begin{tabular}{|c|c|c|c|}
\hline (Roth, et al., 2006) & Railroad Operations & $\begin{array}{l}\text { Interview-Based } \\
\text { Study }\end{array}$ & $\begin{array}{l}\text { The research found various informal tactics that workers have developed across } \\
\text { multiple railroad skills as proactive communications that leverage shared } \\
\text { situation awareness across the distributed organization, facilitate work, and } \\
\text { contribute to the overall efficiency, safety, and resilience to errors. }\end{array}$ \\
\hline (Cooren, 2004) & Drug Rehabilitation & $\begin{array}{l}\text { Observational } \\
\text { Study }\end{array}$ & $\begin{array}{l}\text { The findings show that a form of collective intelligence can be found more } \\
\text { generally in patterns of conversational behaviors (representation, contribution, } \\
\text { and subordination). The managers are shown to be constructing, amending, and } \\
\text { adding a series of textual blocks that ultimately represent the group's } \\
\text { heedfulness. }\end{array}$ \\
\hline (Klein, et al., 2006) & Health Care & $\begin{array}{l}\text { Observational } \\
\text { Study }\end{array}$ & $\begin{array}{l}\text { The study found that "dynamic delegation" advances action teams' high ability } \\
\text { to perform reliably while also building their newcomer team members' skills. } \\
\text { Further, we suggest that extreme action teams and other "improvisational" } \\
\text { organizational units may achieve swift coordination and reliable performance } \\
\text { by melding hierarchical and bureaucratic role-based structures with flexibility- } \\
\text { enhancing processes. }\end{array}$ \\
\hline (Weick \& Roberts, 1993) & Aircraft Carrier & $\begin{array}{l}\text { Observational } \\
\text { Study }\end{array}$ & $\begin{array}{l}\text { Reliable performance may require a well-developed collective mind in the form } \\
\text { of a complex, attentive system tied together by trust, which seems simple; } \\
\text { however, traditional understanding appears to favor a different configuration: a } \\
\text { simple, automatic system tied together by suspicion redundancy. }\end{array}$ \\
\hline (Casler, 2014) & Aerospace & Case Study & $\begin{array}{l}\text { Based on evaluating NASA concerning a ten dimensions framework, the study } \\
\text { concluded that NASA is consistent with high reliability regarding effectiveness. } \\
\text { Yet, efficiency and representativeness are counter to the developed model- } \\
\text { based HROs. NASA can be seen to show features of an HRO only concerning } \\
\text { the degree of risk involved in operations and the complexity of operational } \\
\text { processes. }\end{array}$ \\
\hline $\begin{array}{l}\text { (Busby \& Iszatt-White, } \\
\text { 2014) }\end{array}$ & Construction & Case Study & $\begin{array}{l}\text { The study identified four main types of understanding construction workers } \\
\text { have about reliability, namely, (1) confirmative; (2) performative; (3) adaptive; } \\
\text { and (4) informative understanding. }\end{array}$ \\
\hline
\end{tabular}




\begin{tabular}{|c|c|c|c|}
\hline (Goldenhar, et al., 2013) & Health Care & $\begin{array}{l}\text { Interview-Based } \\
\text { Study }\end{array}$ & $\begin{array}{l}\text { The study based on the five themes model proposes that huddle leads to } \\
\text { enhanced competencies and quality of information sharing, improved levels o } \\
\text { responsibility, empowerment, and a sense of community. Together, these } \\
\text { cultural attributes develop a culture of collaboration and trust that improves the } \\
\text { workforce quality of collective perception and improved capability for reducing } \\
\text { patient harm. }\end{array}$ \\
\hline (Høyland, et al., 2018) & $\begin{array}{l}\text { Health Care } \\
\text { and } \\
\text { Construction }\end{array}$ & $\begin{array}{l}\text { Interview-Based } \\
\text { Study }\end{array}$ & $\begin{array}{l}\text { HROT principles were found in both sectors, as they appear strongly tied to } \\
\text { informal safety mindsets and practices. Safety principles may be subsidiary to } \\
\text { other day-to-day operations and disruptions, which may adversely reduce the } \\
\text { rate of damaging events and accidents across these sectors. }\end{array}$ \\
\hline (Cox, et al., 2006) & Nuclear/Oil and Gas & Case Study & $\begin{array}{l}\text { The study shows that reducing accidents and near misses, as workers fearec } \\
\text { losing their jobs and apportionment of blame from direct supervisors and } \\
\text { middle managers. }\end{array}$ \\
\hline (Samuels, 2010) & Health Care & Case Study & $\begin{array}{l}\text { The study found that although extensive efforts to improve care, outcome } \\
\text { remain low, and barriers hard to defeat. Change management strategies tha } \\
\text { embrace organizational and individual accountabilities are warranted } \\
\text { The author discusses using a high-reliability framework as } \\
\text { change management strategy. }\end{array}$ \\
\hline $\begin{array}{l}\text { (Mitropoulos \& Cupido, } \\
\text { 2009) }\end{array}$ & Construction & Case Study & $\begin{array}{l}\text { The study recommends that operators focused on error prevention as a crucia } \\
\text { aspect to enhance production and reduce the likelihood of accidents }\end{array}$ \\
\hline $\begin{array}{l}\text { (Vogus \& Sutcliffe, } \\
\text { 2007a) }\end{array}$ & Health Care & $\begin{array}{l}\text { Multisite cross- } \\
\text { sectional Survey- } \\
\text { Based Study }\end{array}$ & $\begin{array}{l}\text { The developed Safety Organizing Scale (SOS), a 9-item unidimensiona } \\
\text { measure of self-reported behaviors, empowered safety culture and wa } \\
\text { observed to have high core reliability and reflect theoretically derived anc } \\
\text { empirically observed content domains. }\end{array}$ \\
\hline $\begin{array}{l}\text { (Vogus \& Sutcliffe, } \\
2007 b \text { ) }\end{array}$ & Health Care & $\begin{array}{l}\text { Survey-Based } \\
\text { Study }\end{array}$ & $\begin{array}{l}\text { The study revealed an indication that the benefits of safety organizing or } \\
\text { reported errors were improved when combined with high levels of trust in the } \\
\text { manager or the use of care pathways. }\end{array}$ \\
\hline
\end{tabular}




\begin{tabular}{|c|c|c|c|}
\hline (Valentine, et al., 2010) & $\begin{array}{l}\text { Education-Based } \\
\text { Healthcare }\end{array}$ & $\begin{array}{l}\text { Survey-Based } \\
\text { Study }\end{array}$ & $\begin{array}{l}\text { The results showed that mindfulness was related to decreased role conflict and } \\
\text { that perceived ethical values and a shared ethics code were related to reduced } \\
\text { role conflict and increased mindfulness. Therefore, companies might better } \\
\text { manage role conflict through the development of mindfulness and } \\
\text { organizational ethics. }\end{array}$ \\
\hline (Busby, 2006) & Railway & Case Study & $\begin{array}{l}\text { Based on the study, future work in this area (i.e., organizational mindfulness in } \\
\text { the railway industry) could profit from further analyses of organizations whose } \\
\text { reliability is marginal rather than ultimately good enough or scarce. What } \\
\text { hinders reliability-seeking organizations is likely to be most apparent in such } \\
\text { settings. }\end{array}$ \\
\hline (Barrett, et al., 2006) & Fire Department & $\begin{array}{l}\text { Survey-Based } \\
\text { Study }\end{array}$ & $\begin{array}{l}\text { Although analysis neither aggregates the measure nor validate it, some } \\
\text { employee showed some supports regarding two factors: self-efficacy and } \\
\text { organizational risk responsiveness. }\end{array}$ \\
\hline (Hoy, et al., 2006) & Middle Education & $\begin{array}{l}\text { Survey-Based } \\
\text { Study }\end{array}$ & $\begin{array}{l}\text { The study concluded that the five elements of mindfulness support } \\
\text { administrators pursue to improve teaching and learning. Innovative educators } \\
\text { who challenge others to seek latent failures in the system will achieve higher } \\
\text { levels of mindfulness. }\end{array}$ \\
\hline $\begin{array}{l}\text { (Vogus \& Welbourne, } \\
2003 \text { ) }\end{array}$ & IPO software & $\begin{array}{l}\text { Survey-Based } \\
\text { Study }\end{array}$ & $\begin{array}{l}\text { The study indicates that three examined HR practices (the use of skilled } \\
\text { temporary employees, positive employee relations, and an emphasis on } \\
\text { training) help a subset of reliability-seeking organizations (IPO software firms) } \\
\text { to achieve innovation. Future research should use both HR practices and the } \\
\text { collective mindfulness framework to study an even wider variety of settings to } \\
\text { assess better the relationships between structures and processes and the scope } \\
\text { and boundary conditions of mindfulness. }\end{array}$ \\
\hline (Ray, et al., 2011) & Higher Education & $\begin{array}{l}\text { Survey-Based } \\
\text { Study }\end{array}$ & $\begin{array}{l}\text { The study examined organizational mindfulness in U.S. business schools and } \\
\text { provided evidence of the five dimensions of organizational mindfulness } \\
\text { articulated by Weick and Sutcliffe (2001). It also considers the impact of role } \\
\text { on how decision-makers view organizational mindfulness and discuss the } \\
\text { implications of our findings for both researchers and managers. }\end{array}$ \\
\hline
\end{tabular}




\begin{tabular}{|c|c|c|c|}
\hline (Anderson, 2010) & $\begin{array}{l}\text { Information } \\
\text { Technology }\end{array}$ & $\begin{array}{l}\text { Survey-Based } \\
\text { Study }\end{array}$ & $\begin{array}{l}\text { The results are consistent with the assertion that it is essential to examine the } \\
\text { effects of collective mindfulness at its dimensions grouped as processes rather } \\
\text { than as an omnibus measure. }\end{array}$ \\
\hline $\begin{array}{l}\text { (Ogliastri \& Zúñiga, } \\
\text { 2016) }\end{array}$ & Higher Education & Case Study & $\begin{array}{l}\text { The leading creator of cases among the top graduate schools of management in } \\
\text { Latin America shows between two and five attributes of extremely mindful } \\
\text { organizations. In Spite Of not being mentioned among academics in Latin } \\
\text { America, Weick's concepts of mindfulness and high reliability organizations } \\
\text { (HROs) are pertinent in an area bound by ambiguity. }\end{array}$ \\
\hline $\begin{array}{l}\text { (Hales \& Chakravorty, } \\
\text { 2016) }\end{array}$ & Health Care & $\begin{array}{l}\text { soft research } \\
\text { methods }\end{array}$ & $\begin{array}{l}\text { This research found that mindfulness used with Soft Systems Methods provides } \\
\text { a useful framework to create HROs. In doing so, this study also discovers a } \\
\text { sixth aspect of HROs. }\end{array}$ \\
\hline (Black \& McBride, 2013) & Wildland Fire & Case Study & $\begin{array}{l}\text { This research raised fundamental questions regarding how HROs key principles } \\
\text { are related to each other structurally and whether the relationships are constant } \\
\text { across the entire system or among different hierarchical or functional sub- } \\
\text { systems. Future research is essential to the linkages between these structures, } \\
\text { their sub-components, and their performance. }\end{array}$ \\
\hline (Muhren, et al., 2007) & Financial Services & $\begin{array}{l}\text { Observational } \\
\text { Study }\end{array}$ & $\begin{array}{l}\text { The findings show that the principles that make an HRO distinct from other } \\
\text { organizations are, to some extent existing in the IT Incident Management } \\
\text { process. The study concluded that substantial opportunities remain uncaptured } \\
\text { to leverage the HRO qualities to a higher level. }\end{array}$ \\
\hline (Jahn \& Black, 2017) & $\begin{array}{l}\text { Wildland } \\
\text { Firefighters }\end{array}$ & $\begin{array}{l}\text { Survey-Based } \\
\text { Study }\end{array}$ & $\begin{array}{l}\text { Results suggest how people can overcome the restriction of the hierarchy } \\
\text { constraints through leaders' communication to nurture an operation's } \\
\text { awareness and how supervisors, members, and groups might boost interactional } \\
\text { cultures with respectful effect. }\end{array}$ \\
\hline $\begin{array}{l}\text { (Borges \& Gonçalo, } \\
2010)\end{array}$ & Electrical System & $\begin{array}{l}\text { Interview-Based } \\
\text { Study }\end{array}$ & $\begin{array}{l}\text { As empirical evidence, there is an interrelationship between micro-processes of } \\
\text { sensemaking, considered as ambiguity, experience, interactions, and the micro- } \\
\text { processes of trust, considered as complexity, familiarity, and social behavior. }\end{array}$ \\
\hline
\end{tabular}




\begin{tabular}{|l|l|l|l|}
\hline (Berardi, 2010) & Fire Department & $\begin{array}{l}\text { Survey-Based } \\
\text { Study }\end{array}$ & $\begin{array}{l}\text { The study revealed a high tendency in the fire department to become an HRO; } \\
\text { however, not all the principles showed promising results, which requires } \\
\text { considering weaknesses and tradeoffs to make the organization fits as a whole. }\end{array}$ \\
\hline (Jahn, 2016) & Wildfire Fighting & $\begin{array}{l}\text { Observational } \\
\text { Study }\end{array}$ & $\begin{array}{l}\text { The findings contribute as an explanatory model for how members adopt safety } \\
\text { rules into action according to workgroup norms, complementary relationships, } \\
\text { and practices. This extends the understanding of adaptive action and learning } \\
\text { in hazardous work organizations. }\end{array}$ \\
\hline $\begin{array}{l}\text { (Hesamamiri, et al., } \\
\text { 2013) }\end{array}$ & $\begin{array}{l}\text { Knowledge } \\
\text { Management }\end{array}$ & $\begin{array}{l}\text { Survey-Based } \\
\text { Study }\end{array}$ & $\begin{array}{l}\text { The reliability evaluation instrument for KM suggested in this study was } \\
\text { constructed with four dimensions only, except reluctant to simplify, which did } \\
\text { not validate reliable factors. The related measurement items were also } \\
\text { identified. }\end{array}$ \\
\hline (Lekka \& Sugden, 2011) & Oil Refinery & Case Study & $\begin{array}{l}\text { The findings illustrate a successful application of reliability-enhancing } \\
\text { practices in several fields, including hazard identification, emergency } \\
\text { preparedness, and analysis of incidents and near misses. Management } \\
\text { commitment to safety emerged as an essential factor behind the successful } \\
\text { implementation. However, fostering a reporting culture and maintaining high } \\
\text { management visibility levels maybe some of the challenges encountered by } \\
\text { organizations. }\end{array}$ \\
\hline (Paranhos, et al., 2017) & Onshore Oil and Gas & Case Study & $\begin{array}{l}\text { The study reveals some characteristics of HRO theory and application among } \\
\text { industry leaders. The studied companies have implemented various HROs } \\
\text { practices focused on workforce protection and total quality management to } \\
\text { strengthen a company's safety culture, improve process safety, and minimize } \\
\text { the risk of catastrophic failures. }\end{array}$ \\
\hline
\end{tabular}




\section{Appendix B: Selected Research Publications}

Table 23: Selected Studies to Conduct the Qualitative Phase of The Research

\begin{tabular}{|c|c|c|c|}
\hline Ref. & Title & $\begin{array}{c}\text { Type of } \\
\text { Publication }\end{array}$ & Author(s) \\
\hline 1 & Organizing for High Reliability: Processes of Collective Mindfulness & Book Chapter & (Weick, et al., 1999) \\
\hline 2 & Managing the Unexpected: Sustained Performance in a Complex World & Book & (Weick \& Sutcliffe, 2015) \\
\hline 3 & High Reliability Organizations (HROs) & Journal Article & (Sutcliffe, 2011) \\
\hline 4 & High Reliability Organizations: A Review of The Literature & Study Report & (Lekka, 2011) \\
\hline 5 & $\begin{array}{l}\text { The Successes and Challenges of Implementing High Reliability Principles: A Case Study of } \\
\text { A UK Oil Refinery }\end{array}$ & Journal Article & (Lekka \& Sugden, 2011) \\
\hline 6 & Doing No Harm: Enabling, Enacting, and Elaborating a Culture of Safety in Health Care & Journal Article & (Vogus, et al., 2010) \\
\hline 7 & Measuring High Reliability Characteristics of the Organization & Study Report & (Berardi, 2010) \\
\hline 8 & A New Perspective on How to Understand, Assess and Manage Risk and The Unforeseen & Journal Article & (Aven \& Krohn, 2014) \\
\hline 9 & Drift, Adaptation, Resilience and Reliability: Toward an Empirical Clarification & Journal Article & (Pettersen \& Schulman, 2016) \\
\hline 10 & Re-Examining High Reliability: Actively Organizing for Safety & Journal Article & (Sutcliffe, et al., 2017) \\
\hline 11 & The Affective Foundations of High-Reliability Organizing & Journal Article & (Vogus, et al., 2014) \\
\hline 12 & Trust in High-Reliability Organizations & Journal Article & (Schöbel, 2009) \\
\hline 13 & High Reliability Organizations and Mindful Leadership & $\begin{array}{l}\text { Conference } \\
\text { Proceedings }\end{array}$ & (Mellor, et al., 2015) \\
\hline 14 & Organizational Culture in High Reliability Organizations: An Extension & Journal Article & (Klein, et al., 1995) \\
\hline 15 & High Reliability Organizations: Unlikely, Demanding and At Risk & Journal Article & (La Porte, 1996) \\
\hline
\end{tabular}




\begin{tabular}{|c|c|c|c|}
\hline 16 & Working in Practice but Not in Theory: Theoretical Challenges of HROs & Journal Article & (LaPorte \& Consolini, 1991) \\
\hline 17 & $\begin{array}{l}\text { Learning from What Do You Do When You Build Safety? Practitioners' Guide to Learning } \\
\text { from Successful Operations }\end{array}$ & Study Report & (Rosness, et al., 2016) \\
\hline 18 & Learning in High Reliability Organizations (HROs): Trial Without Error & $\begin{array}{l}\text { Conference } \\
\text { Proceedings }\end{array}$ & (Aase \& Tjensvoll, 2003) \\
\hline 19 & Learning Process Promoted by Sensemaking and Trust: A Study Related to Unexpected & Journal Article & (Borges \& Gonçalo, 2010) \\
\hline 20 & The Underappreciated Role of Habit in Highly Reliable Healthcare & Journal Article & (Vogus \& Hilligoss, 2016) \\
\hline 21 & Mindfulness in Organizations: A Cross-Level Review & Journal Article & (Sutcliffe, et al., 2016) \\
\hline 22 & Teamwork as an Essential Component of High-Reliability Organizations & Journal Article & (Baker, et al., 2006) \\
\hline 23 & The High Reliability Organization Perspective & Book Chapter & (Dekker \& Woods, 2010) \\
\hline 24 & Huddling for High Reliability and Situation Awareness & Journal Article & (Goldenhar, et al., 2013) \\
\hline 25 & $\begin{array}{l}\text { Theoretical and Operational Challenges of "High-Reliability Organizations": Air-Traffic } \\
\text { Control and Aircraft Carriers }\end{array}$ & Journal Article & (La Porte \& Consolini, 1998) \\
\hline 26 & Toward High Reliability Project Organizing in Safety-Critical Projects & Journal Article & (Saunders, 2015) \\
\hline 27 & Becoming a High Reliability Organization: Operational Advice for Hospital Leaders & Study Report & (Hines, et al., 2008) \\
\hline 28 & $\begin{array}{l}\text { A Model of Communicative and Hierarchical Foundations of High Reliability Organizing in } \\
\text { Wildland Firefighting Teams }\end{array}$ & Journal Article & (Jahn \& Black, 2017) \\
\hline 29 & $\begin{array}{l}\text { Fair and Just Culture, Team Behavior, and Leadership Engagement: The Tools to Achieve } \\
\text { High Reliability }\end{array}$ & Journal Article & (Frankel, et al., 2006) \\
\hline 30 & $\begin{array}{l}\text { Mitigating Hazards Through Continuing Design: The Birth and Evolution of a Pediatric } \\
\text { Intensive Care Unit }\end{array}$ & Journal Article & (Madsen, et al., 2006) \\
\hline 31 & Collective Mind in Organizations: Heedful Interrelating on Flight Decks & Journal Article & (Weick \& Roberts, 1993) \\
\hline 32 & Creating High Reliability Organizations Using Mindfulness & Journal Article & (Hales \& Chakravorty, 2016) \\
\hline
\end{tabular}




\begin{tabular}{|c|c|c|c|}
\hline 33 & Organizing for Transient Reliability: The Production of Dynamic Non-Events & Journal Article & (Weick, 2011) \\
\hline 34 & High Reliability Leadership: A Conceptual Framework & Journal Article & (Martınez-Corcoles, 2017) \\
\hline 35 & Assessing Your Organization's Potential to Become a High Reliability Organization & Journal Article & (Youngberg, 2004) \\
\hline 36 & Overcoming Dysfunctional Momentum: Organizational Safety as A Social Achievement & Journal Article & (Barton \& Sutcliffe, 2009) \\
\hline 37 & $\begin{array}{l}\text { An empirical exploration of the presence of HRO safety principles across the health care sector } \\
\text { and construction industry in Norway }\end{array}$ & Journal Article & (Høyland, et al., 2018) \\
\hline 38 & $\begin{array}{l}\text { Structuring for high reliability: HR practices and mindful processes in reliability-seeking } \\
\text { organizations }\end{array}$ & Journal Article & (Vogus \& Welbourne, 2003) \\
\hline 39 & Organizational Mindfulness in Business Schools & Journal Article & (Ray, et al., 2011) \\
\hline 40 & $\begin{array}{l}\text { IT is risky business: Three essays on ensuring reliability, security, and privacy in technology } \\
\text { mediated settings. }\end{array}$ & PhD Dissertation & (Anderson, 2010) \\
\hline 41 & $\begin{array}{l}\text { Assessing high reliability practices in wildland fire management: an exploration and } \\
\text { benchmarking of organizational culture }\end{array}$ & Study Report & (Black \& McBride, 2013) \\
\hline 42 & Measuring the reliability of knowledge management Instrument development and validation & $\begin{array}{l}\text { Conference } \\
\text { Proceedings }\end{array}$ & (Hesamamiri, et al., 2013) \\
\hline 43 & $\begin{array}{l}\text { Highly Reliable Organizations in the Onshore Natural Gas Sector: An Assessment of Current } \\
\text { Practices, Regulatory Frameworks, and Select Case Studies }\end{array}$ & Study Report & (Paranhos, et al., 2017) \\
\hline 44 & $\begin{array}{l}\text { Role Conflict, Mindfulness, and Organizational Ethics in an Education-Based Healthcare } \\
\text { Institution }\end{array}$ & Journal Article & (Valentine, et al., 2010) \\
\hline 45 & $\begin{array}{l}\text { The Safety Organizing Scale: Development and Validation of a Behavioral Measure of Safety } \\
\text { Culture in Hospital Nursing Units }\end{array}$ & Journal Article & (Vogus \& Sutcliffe, 2007a) \\
\hline 46 & $\begin{array}{l}\text { The Impact of Safety Organizing, Trusted Leadership, and Care Pathways on Reported } \\
\text { Medication Errors in Hospital Nursing Units }\end{array}$ & Journal Article & (Vogus \& Sutcliffe, 2007b) \\
\hline 47 & Aircraft Carrier Operations at Sea: The Challenges of High Reliability Performance & Study Report & (LaPorte, et al., 1988) \\
\hline
\end{tabular}




\section{Appendix C: Labels and Definitions of Final Codes}

Table 24: Final List of Codes Across Five Codes Families

\begin{tabular}{|c|c|c|c|}
\hline $\begin{array}{l}\text { Coding } \\
\text { Family }\end{array}$ & & Code Label & Code Definition \\
\hline & \multirow{4}{*}{ 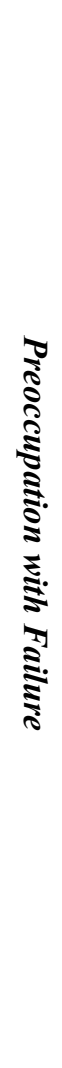 } & $\begin{array}{l}\text { Detecting and } \\
\text { Resolving } \\
\text { Anomalies }\end{array}$ & $\begin{array}{l}\text { It refers to the practice of continual searching for any discrepancies and suspicious cues, catching and resolving } \\
\text { them within a plausible time before they grew into serious problems. An anomaly is an indication of something } \\
\text { wrong that does not fit into a series. Something that is a departure from common order, form, or rule. It has been } \\
\text { described as contrasting in the quality of the successive segments of a stream of thoughts. An anomaly serves as a } \\
\text { motive to examine intentions and expectations. }\end{array}$ \\
\hline & & System Knowledge & $\begin{array}{l}\text { It refers to the knowledge regarding the physical interconnections and parameters of the systems, and how it works } \\
\text { as a whole through knowing; (1) weaknesses in the technologies, (2) slops in the operations, (3) gaps in the } \\
\text { procedure, and (4) the orders by which errors in the system are triggering each other. However, the literature does } \\
\text { not directly connect system knowledge to detecting and resolving anomalies, but the context explains that one can } \\
\text { better detect anomalies when having better system knowledge. }\end{array}$ \\
\hline & & $\begin{array}{l}\text { Expectations } \\
\text { Domain }\end{array}$ & $\begin{array}{l}\text { It refers to the scope of the anticipations surrounding the system, its processes, and operations. Whenever } \\
\text { something comes from outside the frame (domain), it would be considered as an anomaly that requires an action. } \\
\text { The expectation domain is regarded as a tool for sensemaking. }\end{array}$ \\
\hline & & $\begin{array}{l}\text { Cues of Evolving } \\
\text { Failure }\end{array}$ & $\begin{array}{l}\text { It refers to the unnoticed signals (e.g., small errors, near misses), which, when they accumulate, a failure could } \\
\text { evolve. It is also known as the conversion of a cue into a clue. These cues continue to develop until a failure } \\
\text { happens, particularly when people misjudge or misinterpret their evolution. These events are perceived as very } \\
\text { harmful that they may disable the entire system, radically limit its capacity to pursue the goals, and lead to its } \\
\text { destruction. To impede these types of events, the organization should increase failure knowledge and sensitivity to } \\
\text { failure. }\end{array}$ \\
\hline
\end{tabular}




\begin{tabular}{|c|c|c|c|}
\hline & & Failure Knowledge & $\begin{array}{l}\text { It refers to the knowledge regarding how a failure evolves, causing the system or a part of the system to collapse. } \\
\text { Acquiring and sustaining failure knowledge is a critical competency to preclude the noticed cues and latent signals' } \\
\text { accumulations. For this organizational property to be maintained and increased, specific experience and other } \\
\text { knowledge types, particularly system knowledge, are required. Therefore, an HRO develops and sustains } \\
\text { repositories of failure as sources of information such as experts and experienced people and building failure } \\
\text { databases based on failure report and failure update and analysis. }\end{array}$ \\
\hline \multirow{5}{*}{ ț } & \multirow{5}{*}{ 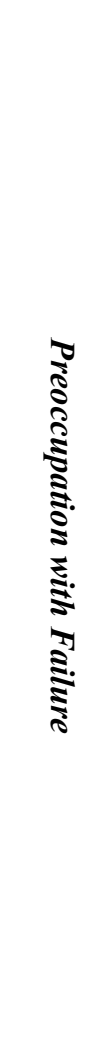 } & Failure Repositories & $\begin{array}{l}\text { It refers to the sources of acquiring failure knowledge through expertise. It has been widely acknowledged that } \\
\text { maintenance departments in each organization play a central or the source of organizational learning. The } \\
\text { accumulation of experience resulted from dealing with a wide range of failures. }\end{array}$ \\
\hline & & Failure Report & $\begin{array}{l}\text { It is denoting to communicate all types of failures by everyone involved regardless of the nature and the size of the } \\
\text { failure; even small and straightforward failures still count as a deviation from the norm. This practice requires a } \\
\text { culture that encourages and rewards, which is derived from the value of keeping the organization fully informed } \\
\text { and aware of any potential cues through increasing failure knowledge. }\end{array}$ \\
\hline & & $\begin{array}{l}\text { Failure Update and } \\
\text { Analysis }\end{array}$ & $\begin{array}{l}\text { It refers to integrating and expanding the failure database through the continual update with any emergent } \\
\text { information and whenever new failures are reported. Updating failure is requiring a continual analysis that diagnose } \\
\text { the symptoms and investigate the causes. This practice increases the failure of knowledge needed to avoid the same } \\
\text { scenarios in the future. }\end{array}$ \\
\hline & & Sensitivity to Failure & $\begin{array}{l}\text { It is defined as the subtlety regarding the system's expected and unexpected failure, which increases the readiness } \\
\text { and responsiveness of the system. The importance of sensitivity is derived from the fact that it is mitigating fixation, } \\
\text { and therefore, it increases the system's capability to reduce the accumulation of any latent cues. }\end{array}$ \\
\hline & & Normalization & $\begin{array}{l}\text { It is defined as tolerating and treating the supposed to be abnormal as normal. For example, accepting or handling } \\
\text { a partial failure or a certain level of risk beyond the standards or the predefined risk is, in fact, normalizing them. } \\
\text { Normalization could happen gradually, such as the phenomenon of creeping change or reliability drift, which } \\
\text { requires excellent attention and adherence to both standards and risk boundaries. }\end{array}$ \\
\hline
\end{tabular}




\begin{tabular}{|c|c|c|c|}
\hline \multirow{5}{*}{ ఫָ } & \multirow{5}{*}{ 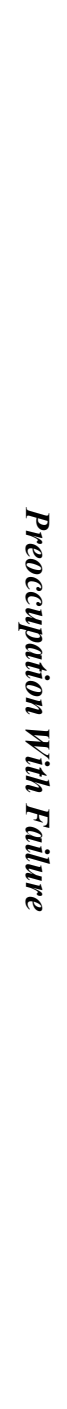 } & $\begin{array}{l}\text { Adherence } \\
\text { Standards }\end{array}$ & $\begin{array}{l}\text { It is defined as the commitments to the precise articulation and definition of the standardized protocols and } \\
\text { checklists and the criteria of the procedures, processes, responsibilities, job descriptions, rules of teamwork and } \\
\text { collaboration, quality of competences, qualifications, and skills, in addition to the training quality and assurance. } \\
\text { The precise and comprehendible articulation of the standards is essential to ensure that all the organization members } \\
\text { have a better understanding of how the system works as a whole and how do they operate within, which guides } \\
\text { them to ensure that they are following and exhibiting safe practices and behaviors. }\end{array}$ \\
\hline & & $\begin{array}{l}\text { Adherence to Risk } \\
\text { Boundaries }\end{array}$ & $\begin{array}{l}\text { It is defined as the commitment to the domain and levels of the established and precisely defined risks and hazards } \\
\text { that can be controlled and managed. However, setting boundaries means to coherently articulate what risk and } \\
\text { hazard are and what is not, why the organization may accept a certain level of risk, and how it can be reduced and } \\
\text { mitigated. Considering that in some situations, accepting a certain level of risk requires more attention since, in } \\
\text { such a situation, the accepted level of risk might be gradually extended to a higher level, particularly when the } \\
\text { operations suffer creeping change. }\end{array}$ \\
\hline & & $\begin{array}{l}\text { Institutionalizing } \\
\text { Wariness }\end{array}$ & $\begin{array}{l}\text { It is defined as integrating a mindset of being continuously worried that failures can happen at any time. This is } \\
\text { very important, especially that successful organizations (in terms of safety or reliability) become less worried, or } \\
\text { in other words, "complacent," which may lead to misestimating reliability in the entire or particular parts of the } \\
\text { organization. Therefore, lingering wariness should be adapted, and skepticism should be encouraged. }\end{array}$ \\
\hline & & $\begin{array}{l}\text { Concern and } \\
\text { Calibrate }\end{array}$ & $\begin{array}{l}\text { It refers to a culture of raising apprehension, or in other words, fearing potential cues that require asking questions } \\
\text { about what is wrong or what could go wrong. This is creating an awareness of vulnerability, which helps in } \\
\text { developing a course of learning. While calibrating is to track, report, compare, feedback, and follow-up. }\end{array}$ \\
\hline & & $\begin{array}{l}\text { Misestimating } \\
\text { Reliability }\end{array}$ & $\begin{array}{l}\text { It refers to the incorrect certainty that interim success is a reflection of long-term reliability. When success is } \\
\text { achieved (i.e., reliability is high), organizations tend to reallocate its resources or limit them (e.g., due to financial } \\
\text { reason; reducing costs), thinking that there are no more needs for such resources to promote a higher level of safety, } \\
\text { which in other words, changes the beliefs regarding reliability. This, in fact, reduces the preoccupation with failure } \\
\text { and drifts to be preoccupied with other goals. }\end{array}$ \\
\hline
\end{tabular}




\begin{tabular}{|c|c|c|c|}
\hline \multirow{3}{*}{$\stackrel{7}{7}$} & \multirow{3}{*}{ 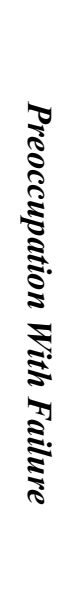 } & Complacency & $\begin{array}{l}\text { It results from ongoing success over time, which eventually leads the organization members, particularly managers, } \\
\text { to be overconfident as they relate success to themselves and demonstrate success as a high competence, ultimately } \\
\text { narrowing their perception towards the unexpected. Complacency is a door for unexpected events to pass through } \\
\text { and occur. }\end{array}$ \\
\hline & & $\begin{array}{l}\text { Institutionalized } \\
\text { Doubt }\end{array}$ & $\begin{array}{l}\text { It is defined as integrating doubt as a mindset considering that dealing with doubts is safer than living with a naïve } \\
\text { certainty. HROs adopt a mindset of doubting that smooth operations do not reflect reliability because dynamic } \\
\text { processes could always bring surprise and unexpected outcomes. This mindset encourages a spirit of contradiction } \\
\text { while discourages the spirit of the accord. }\end{array}$ \\
\hline & & Contradiction Spirit & $\begin{array}{l}\text { It is referring to establishing freedom of posing confronted points of view so that members of the organization urge } \\
\text { and criticize each other when necessary. This spirit has widened the foundation of various inputs that increase the } \\
\text { variety in dealing with the unexpected. }\end{array}$ \\
\hline \multirow{2}{*}{$\vec{v}$} & \multirow{2}{*}{ 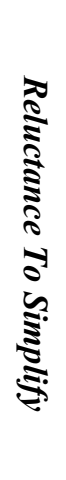 } & Simplification & $\begin{array}{l}\text { It is defined as the tendency to simplify the context that people are working within to understand it or better deal } \\
\text { with it better. Therefore, simplification causes people to miss, neglect, or obscure unwanted, unanticipated, } \\
\text { unexplainable essential details, which increases the probability of producing an unreliable performance. Also, } \\
\text { simplification reduces people the cognitive capacity to comprehend complexity, which puts them in challenging } \\
\text { situations when unexpected happens. }\end{array}$ \\
\hline & & Generalization & $\begin{array}{l}\text { It is defined as the inclination to interpret and treat signals, issues, information, reports, actions, rules, regulations, } \\
\text { etc., based on the general description. Generalization happens when people categorize their world. Thus they } \\
\text { interpret what they notice or perceive based on what they already know or have known. These types of } \\
\text { categorization smoothen over fine-grained distinctions that may predict unexpected. }\end{array}$ \\
\hline 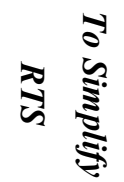 & 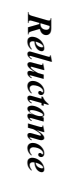 & Misidentification & $\begin{array}{l}\text { It is a product of mistaken identification of an issue based on the identification factors or symptoms of other issues } \\
\text { that have already been identified. This happens when people compare issues superficially to seek similarities for } \\
\text { the sake of simplification, while they neglect the intrinsic differences. Misidentifications could lead to generating } \\
\text { more issues by augmenting the misidentified ones. }\end{array}$ \\
\hline
\end{tabular}




\begin{tabular}{|c|c|c|}
\hline & Distinction & $\begin{array}{l}\text { It refers to differentiating things based on the specific context that they are falling in and the details that they are } \\
\text { providing. Distinctions are highly significant in reducing simplifications, generalizations, and misidentification by } \\
\text { identifying more differences and developing a richer and more diverse image of possible outcomes, suggesting a } \\
\text { richer and more varied set of precautions and early warning signs. }\end{array}$ \\
\hline & $\begin{array}{l}\text { Organizing for } \\
\text { Variety }\end{array}$ & $\begin{array}{l}\text { It is defining the organizational design to increase the process base of sensing, analyzing, and responding to various } \\
\text { inputs. In a complex system, complexity is the only way to cope with the complex environment and sustain a } \\
\text { healthy condition. It has been acknowledged that diverse groups have a wide range of information than those in } \\
\text { homogeneous groups. Organizing for variety can be achieved and sustained through designing requisite variety } \\
\text { and organizational complexity. }\end{array}$ \\
\hline & Requisite Variety & $\begin{array}{l}\text { It is also called requisite complexity. It refers to diversifying the organization members' perspectives over the } \\
\text { traditional means to achieve reliability in a complex environment. For example, experiments showed that a task } \\
\text { managed by people from varied functional, educational, experiential backgrounds is most likely to be successful } \\
\text { than if people of similar backgrounds managed it. }\end{array}$ \\
\hline & $\begin{array}{l}\text { Organizational } \\
\text { Complexity }\end{array}$ & $\begin{array}{l}\text { It refers to the design and the nature of the system structure and the dynamic interactions among its elements when } \\
\text { it functions as a whole. Since HROs design requisite variety to ensure varied inputs, they fabricate complexity to } \\
\text { facilitate that by allowing disagreements and conflicts as normal interactions in HROs that widen the range of } \\
\text { comprehending complexity inherent in such organizations. However, conflicts must be negotiated, managed, and } \\
\text { controlled. These efforts take such forms as diverse checks and balances embedded in the circulation of committees } \\
\text { and meetings, frequent adversarial reviews, selecting new employees with non-typical prior experience, regular } \\
\text { job rotation, and re-training. }\end{array}$ \\
\hline \multirow{2}{*}{ 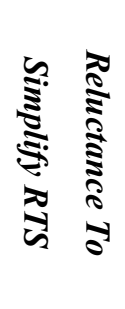 } & Complicated System & $\begin{array}{l}\text { It refers to the system design that is featured by its constituents. A complicated system constitutes negativity since } \\
\text { it encourages simplification resulted from the need to develop a common operational image among individuals. }\end{array}$ \\
\hline & Complex System & $\begin{array}{l}\text { It refers to the system design that is distinct from the interactions among the constituents. A complex system is } \\
\text { desired in HROs since it adapts to requisite complexity. }\end{array}$ \\
\hline
\end{tabular}




\begin{tabular}{|c|c|c|c|}
\hline & & Compilation System & $\begin{array}{l}\text { It refers to the best way to design and organize for a variety that is required to ensure requisite complexity since it } \\
\text { creates emergent complexity and the use of the existing one. For example, when two members interact and make } \\
\text { sense of the nonobvious in ways that neither of them alone could have done. }\end{array}$ \\
\hline & & Ad hoc Networks & $\begin{array}{l}\text { They are defined as informal latent and complex networks created and activated to encounter certain situations that } \\
\text { take the normal operations to where they get outside the boundaries and the anticipation's scope. Such networks } \\
\text { are created as a supplement to the normal patterns of formal hierarchy and compliance with strict roles to solve the } \\
\text { issue and dissolved after the problem is solved. }\end{array}$ \\
\hline & & $\begin{array}{l}\text { Organizing for } \\
\text { Sense-Discrediting }\end{array}$ & $\begin{array}{l}\text { It refers to embedding uncertainties regarding the existing concepts in confronting dynamic, complex } \\
\text { environments, which means organizing for more openness. In other words, the system is open to new information } \\
\text { and new experiences, by which the system is capable of coping with such environments. Systems confronted with } \\
\text { the dynamic complex environment need to be able to develop brilliant ideas about that environment (sense-making) } \\
\text { and criticize their ideas (sense-discrediting). Therefore, organizing for Sense-Discrediting enables the organization } \\
\text { to slow simplifications and allow for simultaneous change and stability. That is why sense-discrediting functions } \\
\text { as a balancing force. }\end{array}$ \\
\hline \multirow{3}{*}{$\vec{\omega}$} & \multirow{3}{*}{ 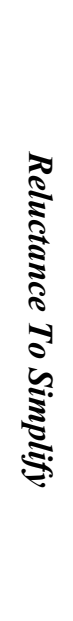 } & Redundancy & $\begin{array}{l}\text { It is defined as a designed form of duplications and backups in the system. It is a backup for technological, } \\
\text { individuals, and organizational elements. Redundancy takes a form of skepticism, which may frustrate any type of } \\
\text { complacency, and involves cross-checks, doubts that precautions are sufficient, and wariness about claimed levels } \\
\text { of competence. Redundancy can make the system more complex to get oversimplifications and misidentifications } \\
\text { by making the system denser and varied. }\end{array}$ \\
\hline & & $\begin{array}{l}\text { Actions } \\
\text { Interpretation }\end{array}$ & $\begin{array}{l}\text { It refers to translating actions into more understandable information extracted from the actions of those who solve } \\
\text { problems and interpret that for learning. }\end{array}$ \\
\hline & & $\begin{array}{l}\text { Continual } \\
\text { Reassessment of } \\
\text { Diagnoses }\end{array}$ & $\begin{array}{l}\text { It refers to the frequent examination and evaluation of the credibility of the available diagnoses. This process } \\
\text { continues until these diagnoses make sense or keep looking for more options. This process reduces the vulnerability } \\
\text { of producing misjudgments. }\end{array}$ \\
\hline
\end{tabular}




\begin{tabular}{|c|c|c|c|}
\hline & & $\begin{array}{l}\text { Cultivation of } \\
\text { Alternatives } \\
\text { Diagnoses }\end{array}$ & $\begin{array}{l}\text { It refers to collecting more options by expanding the boundaries to consider a broader set of alternative diagnoses, } \\
\text { enhancing the anticipation and flexibility needed for mindful organizing. }\end{array}$ \\
\hline$\frac{5}{0}$ & 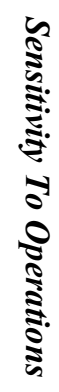 & $\begin{array}{l}\text { Anchoring in the } \\
\text { Present }\end{array}$ & $\begin{array}{l}\text { It is defined as the continual concentration and the full mind's use on the operations within the present moment } \\
\text { that the operations are ongoing. That is because operations are described being acting in real-time, where the } \\
\text { momentary context, complete with its content and feelings, will affect people's behaviors (actions). Anchoring in } \\
\text { the present increases attentiveness and readiness for quick responses. However, deviance of the attention or } \\
\text { attention wavers would lead to lapses that eventually cause operators to lose control and preclude their capacity to } \\
\text { detect and resolve anomalies and cues of evolving failures. Anchoring in the present requires a clear mind, which } \\
\text { is described as the mind that takes the form of thinking while acting, thinking by acting, and thinking through } \\
\text { acting. }\end{array}$ \\
\hline & \multirow{4}{*}{ 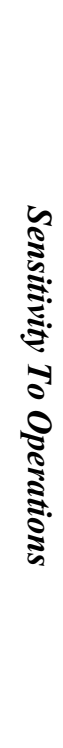 } & Ignorance & $\begin{array}{l}\text { It refers to neglect and apathy, both intentionally or unintentionally, to advice or a safe direction. This could be a } \\
\text { result of self-complacency or personal culture. }\end{array}$ \\
\hline & & Casualness & $\begin{array}{l}\text { It refers to people's tendency to ease and simplify situations and issues around the context they work within. } \\
\text { Casualness leads to superficial interpretations when it is essential to interpret the signals and actions required to } \\
\text { diagnose what is happening and act thoughtfully. }\end{array}$ \\
\hline & & Distraction & $\begin{array}{l}\text { It refers to whatever reduces mind concentration and takes it elsewhere than the work in the present moment. } \\
\text { Anchoring in the present requires full attention and be with all mind, which is described as the mind takes the form } \\
\text { of thinking while acting, thinking by acting, and thinking through acting. }\end{array}$ \\
\hline & & $\begin{array}{lr}\text { Constructing and } \\
\text { Sustaining the } \\
\text { Cognitive Map }\end{array}$ & $\begin{array}{l}\text { It refers to constructing and maintaining the cognitive map that allows people to integrate diverse inputs, } \\
\text { information sensors and remote observation, and real-time status and performance. This is called "having the } \\
\text { bubble" in US Navy ships. This is very important, mainly to cope with the surprise of the automated operations. }\end{array}$ \\
\hline
\end{tabular}




\begin{tabular}{|c|c|c|c|}
\hline & & $\begin{array}{l}\text { Building Plausible } \\
\text { Stories }\end{array}$ & $\begin{array}{l}\text { It is referring to the assembly of complex inputs, references, explanations, and definitions of the total situation. For } \\
\text { example, operators build stories to see "whether actions indicated in the procedure steps make sense in the context } \\
\text { of a particular event." Building stories are sustaining the cognitive maps required to stay sensitive to the operations. }\end{array}$ \\
\hline & \multirow{6}{*}{ 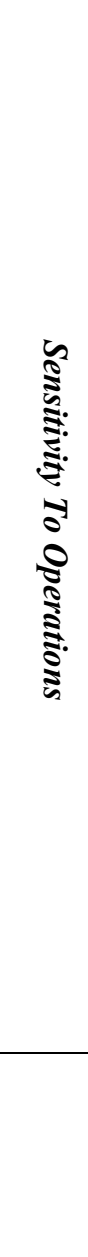 } & $\begin{array}{l}\text { Maintaining Real- } \\
\text { time Situational } \\
\text { Awareness }\end{array}$ & $\begin{array}{l}\text { Situational awareness is defined as the awareness of the environment's components within a time and space, the } \\
\text { understanding of their significance, and the forecast of their status shortly. Therefore, this pattern is denoting to } \\
\text { upholding the situational awareness during the real-time operations, which means to reduce or mitigate any laps } \\
\text { between perception and ongoing operations. }\end{array}$ \\
\hline & & $\begin{array}{l}\text { Assessment and } \\
\text { Continual Updates }\end{array}$ & $\begin{array}{l}\text { It refers to the constant appraisal of the operators' cognition representation with any new emergent inputs, } \\
\text { references, interpretations, and definitions to assess their situational awareness. }\end{array}$ \\
\hline & & $\begin{array}{l}\text { Procedure } \\
\text { Knowledge }\end{array}$ & $\begin{array}{l}\text { It is referring to the logic of the series of actions conducted in a specific order or manner to execute the operations. } \\
\text { Operators use knowledge of the assumptions and logic that underlie preplanned procedures to deal with situations } \\
\text { not fully covered by the procedure. }\end{array}$ \\
\hline & & $\begin{array}{l}\text { Cognitive } \\
\text { Limitations }\end{array}$ & $\begin{array}{l}\text { It refers to individuals' cognition resources to process and integrate a map and build stories of real-time awareness } \\
\text { and assessment. Individuals' cognitive capacity is naturally limited and possibly reduced even more when } \\
\text { individuals experience stress, fatigue, distraction, and other job-related effects. When ongoing operations do not } \\
\text { fully occupy cognitive capacity, maintaining situational awareness in real-time becomes an issue, and laps often } \\
\text { occur, which dangers the reliable outcome. }\end{array}$ \\
\hline & & $\begin{array}{l}\text { Situational } \\
\text { Awareness Drifts }\end{array}$ & $\begin{array}{l}\text { It refers to the loss of situational awareness during real-time operations, which, when happens, operators fail to } \\
\text { notice the error and detect anomalies. At the same time, continue to be guided by inaccurate simplifications, sunken } \\
\text { in intentions and plans, rely on an established routine, and follow authorities blindly. }\end{array}$ \\
\hline & & $\begin{array}{l}\text { Heedful } \\
\text { Interrelating }\end{array}$ & $\begin{array}{l}\text { Heed is defined as attending to how something is achieved while doing it. Heedful interrelating then refers to a } \\
\text { collective mind or social process through which individual action participates in a larger shared action pattern and } \\
\text { understands how their efforts fit into that more extensive pattern. This level of heedfulness explains how the } \\
\text { operations are tied together by patterns shaping the members' participation in the organization's total outcome, }\end{array}$ \\
\hline
\end{tabular}




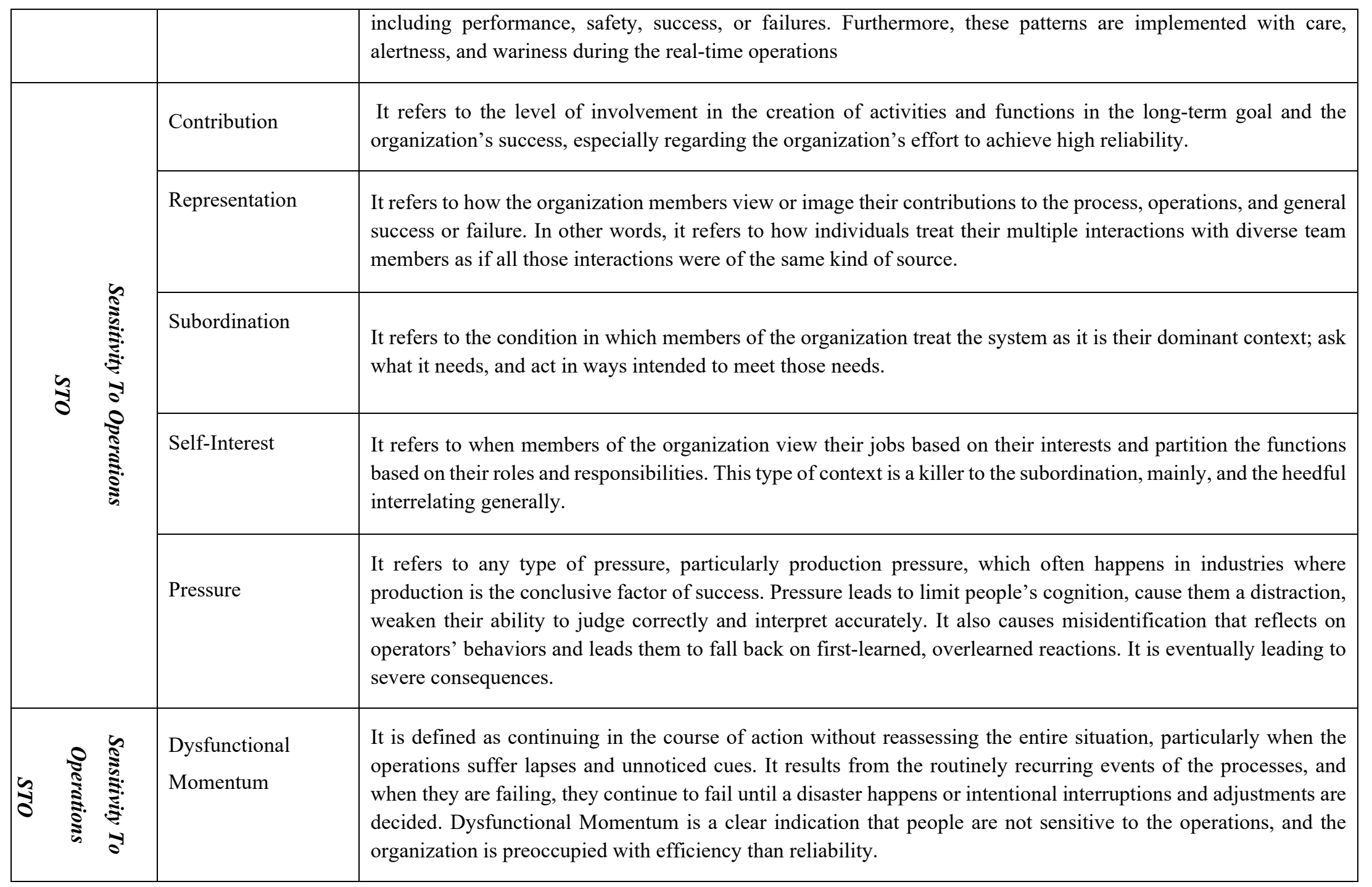

U 


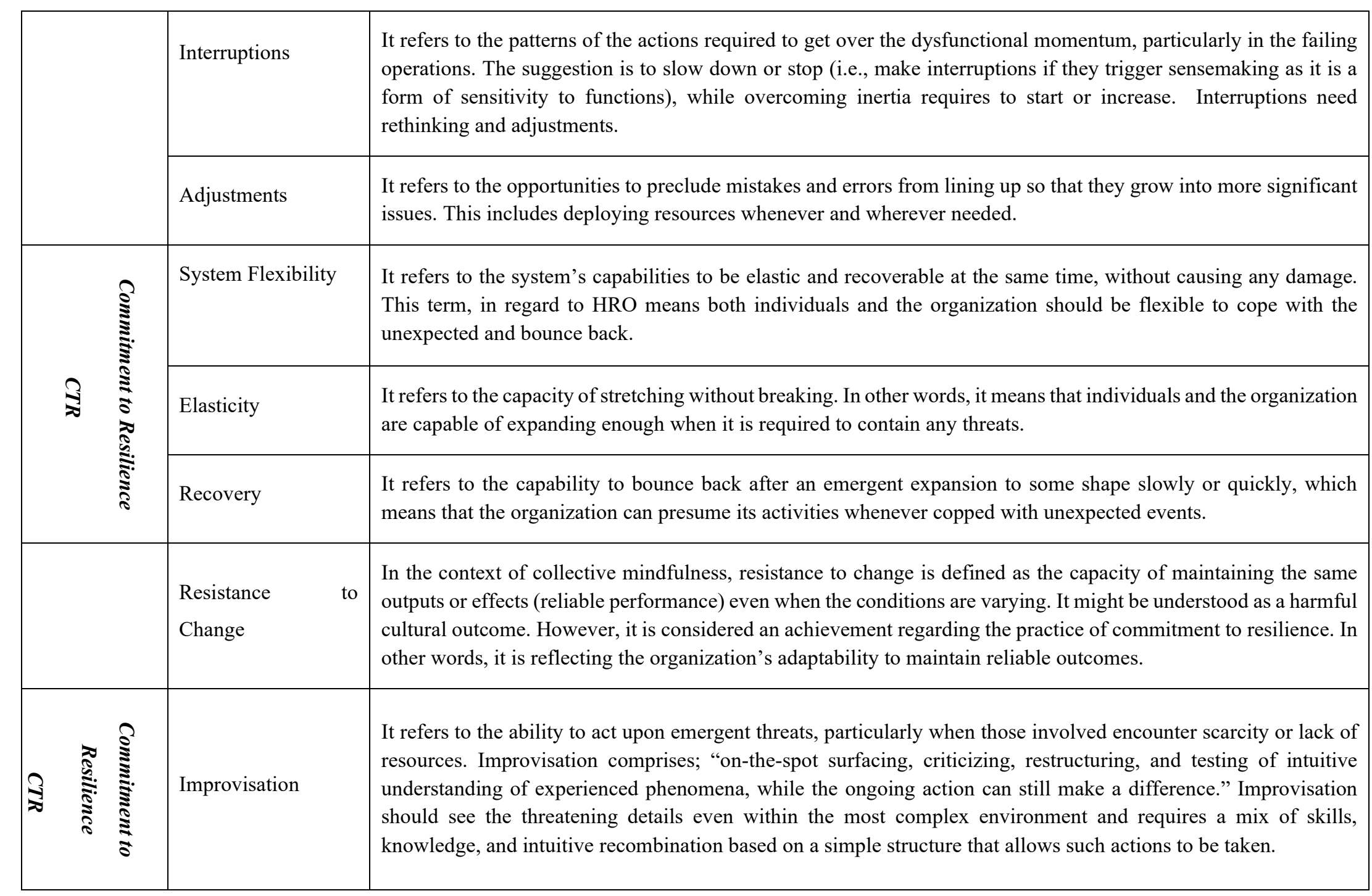

N 


\begin{tabular}{|c|c|c|c|}
\hline & Simple Structure & $\begin{array}{l}\text { It refers to the preexisting or minimal structure that provides experienced people with a frame to act (improvise) } \\
\text { within. This simple structure could be the set of the core values, regulations, rules of engagement, or the mission } \\
\text { statement, within which people can direct themselves and engage with sensemaking. }\end{array}$ \\
\hline & & $\begin{array}{l}\text { General } \\
\text { Knowledge }\end{array}$ & $\begin{array}{l}\text { Refers to knowing concepts, principles, rules, regulations, standards, and boundaries regarding the system, } \\
\text { procedure, expectations, and failures throughout all the available resources. }\end{array}$ \\
\hline & & Interpersonal Skills & $\begin{array}{l}\text { It refers to exceptional personal capabilities to apply the information and knowledge and the received training in } \\
\text { the job's specific context. }\end{array}$ \\
\hline & & Confidence & $\begin{array}{l}\text { The feel of doing so. It is a product of knowing the self and the personal capabilities and the encouragement, } \\
\text { motivation, and trust by upper management. }\end{array}$ \\
\hline & & Sensemaking & $\begin{array}{l}\text { It refers to the process of social construction that creates a workable level of certainty and imposes order on } \\
\text { perceptions, experiences, and expectations. Sensemaking is about thinking while acting, and it is involving the } \\
\text { ongoing reflective development of plausible images that validate what people are doing and experiencing. } \\
\text { Sensemaking highlights that people try to make things reasonably accountable to themselves and others; therefore, } \\
\text { to make sense of things during a trying condition, people need to reconstitute the evolving present through } \\
\text { continuous assessments and calibrations. }\end{array}$ \\
\hline & \multirow{2}{*}{ 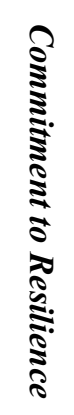 } & $\begin{array}{l}\text { Reconstituting the } \\
\text { Evolving Present }\end{array}$ & $\begin{array}{l}\text { It is the process of recalibrating the expectations in real-time. When an unexpected event happens, individuals and } \\
\text { organizations face an evolving present that might never be experienced before (i.e., no previous similar situations } \\
\text { to learn from, and not enough or a total lack of resources to depend on. Therefore, such situations require the } \\
\text { development of solutions in parallel with the evolving problems, or in other words, those involved use different } \\
\text { modes, find new dynamics and develop new logic. }\end{array}$ \\
\hline & & $\begin{array}{l}\text { Communication and } \\
\text { Collaboration }\end{array}$ & $\begin{array}{l}\text { It refers to the required, frequent and intensive cooperated exchanges and share of the information, warning signs, } \\
\text { news, wisdom, expertise, advice, etc., to developmental representations and mutual knowledge among all }\end{array}$ \\
\hline
\end{tabular}




\begin{tabular}{|c|c|c|c|}
\hline & \multirow[b]{2}{*}{$\begin{array}{l}\text { Responses (Actions) } \\
\text { Repertoire }\end{array}$} & \multirow{2}{*}{$\begin{array}{l}\text { organization members. Communication and collaboration lead to developing a social force and engagement, which } \\
\text { strengthens heedfulness and accountability. } \\
\text { It is an extensive and rich reference for developing capabilities for commitment to resilience built through training } \\
\text { and simulation, varied job experiences, learning from negative feedback, and ad hoc networks that allow for rapid } \\
\text { pooling of expertise to handle unanticipated events. Responses or action repertoire include different levels of } \\
\text { information richness, collaborative practice, and pro-active focus. Organizational knowledge within HROs should } \\
\text { be considered by a comprehensive action repertoire to redefine investigation and learning by trial-and-error and a } \\
\text { high degree of hands-on focus to get ready for unexpected incidents. }\end{array}$} \\
\hline & & & \\
\hline & & $\begin{array}{l}\text { Learning } \\
\text { Failure }\end{array}$ & $\begin{array}{l}\text { It is considered a key factor to operate resiliently. It refers to expanding the information already generated from } \\
\text { previous errors (failure) and implementing that learning through fast and negative feedback. This component is } \\
\text { recently labeled "Learning from incident LFI" and was giving very close attention from academia and practitioners. } \\
\text { This type of learning enlarges the response repertoire and highly and positively increase the capability of } \\
\text { commitment to resilience. }\end{array}$ \\
\hline \multirow[b]{2}{*}{ 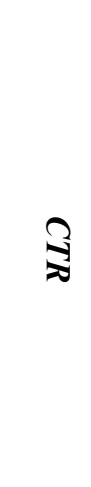 } & \multirow{2}{*}{ 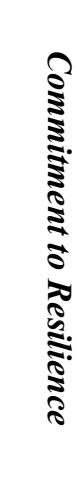 } & Negative Feedback & $\begin{array}{l}\text { It is fast-real-time learning that allows people to cope with an unfolding surprise in ways that are not specified in } \\
\text { advance. It is a base of the learning process from failure, whereby operators tend to end or reduce the fluctuations } \\
\text { in the output failures or errors. It is sometimes called double-loop learning. }\end{array}$ \\
\hline & & Training & $\begin{array}{l}\text { Mindful training or training for resilience can be defined as a set of practices focused on teamwork with equal } \\
\text { concentration on cognitive processing and interpersonal skills. It should be designed to create team members who } \\
\text { consistently use sensory judgments, make quality decisions, and access all required resources under stressful } \\
\text { conditions in a time-constrained environment. It is suggested that training may cover the following aspects: } \\
\text { situational awareness, group dynamics, effective communications, risk management, decision-making workload } \\
\text { management, stress awareness and management, mission planning, physiology, and human performance. }\end{array}$ \\
\hline & $\frac{3}{8}$ & $\begin{array}{l}\text { Migrating Decision } \\
\text { Making }\end{array}$ & $\begin{array}{l}\text { It is the process of pushing down the decision-making authority to the people (individuals or teams) in the lower } \\
\text { and lowest levels of the organization, particularly to those who have specific knowledge around certain events } \\
\text { when required to make faster and effective decisions. It is often happening when people in lower levels notice }\end{array}$ \\
\hline
\end{tabular}




\begin{tabular}{|c|c|c|}
\hline & & $\begin{array}{l}\text { potential failures that need to take immediate actions and decisions at their ends to mitigate any consequences or } \\
\text { reduce their harmful effects. }\end{array}$ \\
\hline & Structure Flexibility & $\begin{array}{l}\text { It is denoting the property of loosening or untying the hierarchical structure's rigidity to allow for the decision- } \\
\text { making process to migrate to lower levels, particularly to those in frontlines during situations of emergencies and } \\
\text { serious threats. In a mindful system, the structure is a variable, but the activity of structuring is constant. This } \\
\text { property requires collective cultural beliefs that the required competence lies somewhere in the system and that } \\
\text { migrating problems will find them. These beliefs are associated with a more mindful recruitment of expertise. }\end{array}$ \\
\hline \multirow{4}{*}{ 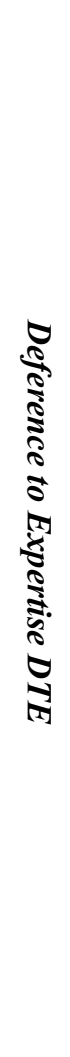 } & Respectful Yielding & $\begin{array}{l}\text { It is denoting to the nature of the interactions or subordination among the members of the organization, which is } \\
\text { described to happen relative to the performance criteria applied in a particular context, or other words, it happens } \\
\text { to interpret an action, answer a question, solve a problem, provide advice and so on. Respectful yielding as a } \\
\text { property of deference to expertise reflects its nature as relational rather than an individual. It is considered as the } \\
\text { foundation of the shared understanding and communication of interpretations. }\end{array}$ \\
\hline & Self-Awareness & $\begin{array}{l}\text { It refers to knowing and acknowledging the knowledge and experiences of the self and the limits and gaps in them. } \\
\text { Therefore, a real expert is not hesitant to inquire about others' knowledge when he/she needed. }\end{array}$ \\
\hline & Available Expertise & $\begin{array}{l}\text { Expertise is a co-production of modified, varied, and sometimes conflicted opinions, sources of supplying data, } \\
\text { mixed experiences, and additional inputs to discussions and conversations. Experts can make sense because they } \\
\text { pay close attention to context and are known to solve problems or answer questions relating to a specific problem- } \\
\text { solving domain or area of expertise. In HROs, those experts are termed as reliability professional "RPs," who are } \\
\text { generally part of teams, have special perspectives on reliability, both cognitively and normatively. They have been } \\
\text { termed "reliability professionals," although they need not be holders of particular positions, have professional } \\
\text { degrees, or even higher degrees. They can be found at all levels of HROs - some are top-level executives. Still, } \\
\text { they can be found among department heads, program managers, shift supervisors, and on down to control operators, } \\
\text { pilots, nurses and maintenance engineers, and technicians. To grasp the right and relative expertise, one needs to } \\
\text { avoid self-importance, equate expertise to experience, and authority. }\end{array}$ \\
\hline & Self-Importance & $\begin{array}{l}\text { It refers to a false rank that some people claim as they pursue central positions or conduct sensitive works due to } \\
\text { the level of the risk inherent in their jobs, which might be deceptively confirmed when others tell them that they }\end{array}$ \\
\hline
\end{tabular}




\begin{tabular}{|c|c|c|}
\hline & & $\begin{array}{l}\text { are essential. However, such responsibilities do not mean that these experts are all-knowing. The problem lies when } \\
\text { others mistakenly hide important information assuming that these so-called experts already know it, while they } \\
\text { may not. Therefore, in solving problems, people respectfully defer to the wrong experts. }\end{array}$ \\
\hline \multirow{5}{*}{ 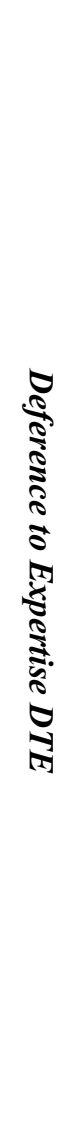 } & $\begin{array}{l}\text { Equating Expertise } \\
\text { to Experience }\end{array}$ & $\begin{array}{l}\text { It is denoting the mistaken beliefs that expertise can be found in those with more years of experience. People falsely } \\
\text { believe that the more years others spent in a specific job, the more experienced they are. Reliant expertise is } \\
\text { minimized in such situations since people with less experience would defer to the wrong experts. }\end{array}$ \\
\hline & $\begin{array}{l}\text { Equating Expertise } \\
\text { to Authority }\end{array}$ & $\begin{array}{l}\text { It is denoting the mistaken beliefs that a higher rank or a higher authority is a reflection of expertise, and therefore, } \\
\text { people yield to the so-called wrong experts based on their rank. In fact, this view narrows the significance of } \\
\text { deference to expertise and minimizes true expertise in the organization. }\end{array}$ \\
\hline & $\begin{array}{l}\text { Domain-Specific } \\
\text { Knowledge }\end{array}$ & $\begin{array}{l}\text { It is defined as a firsthand and simulated experience. This knowledge is relevant to a specific domain that is an } \\
\text { environment or situation or problems' category. Domain-specific knowledge is what constitutes an expert. Much } \\
\text { problem solving involves domain-specific knowledge. This property reflects that there is "little transfer from high- } \\
\text { level proficiency in one domain to proficiency in other domains-even when the domains seem, intuitively, very } \\
\text { similar." }\end{array}$ \\
\hline & Experience & $\begin{array}{l}\text { It is defined as; "we know with what we have known." This simple definition implies that what we have known } \\
\text { comprises some understanding of the nature and functioning of the expertise we know to whom we defer. In } \\
\text { addition, it includes experience with specific unexpected events. Firsthand experience in a specific domain is more } \\
\text { or less meaningful, depending on the frequency and depth of that experience. }\end{array}$ \\
\hline & $\begin{array}{l}\text { Compressed } \\
\text { Experience }\end{array}$ & $\begin{array}{l}\text { It is the product of experience-based knowledge and what clarifies expertise and defines the core of the expert's } \\
\text { role. In other words, an expert who spent a certain period and made efforts in a specific domain knows better than } \\
\text { those whom their experiences are in a different domain. However, knowledge in this context is attainable when } \\
\text { people are willing to spend more time and effort to gain the necessary experience. }\end{array}$ \\
\hline
\end{tabular}




\begin{tabular}{|l|l|l|}
\hline & Experience Depth & $\begin{array}{l}\text { It is the product of experience in a specific domain that is built by handling contextual risks and stresses. Sufficient } \\
\text { expertise is built up as people learn the lessons of closure or learn that even bad events come to an end, which } \\
\text { people could learn from. }\end{array}$ \\
\hline \multirow{2}{*}{$\begin{array}{l}\text { It refers to the experience of recurrence and density in a specific domain (i.e., experience depth). For example, } \\
\text { experiencing a domain or specific emergency once or twice is not enough to understand or cope with a specific } \\
\text { environment. Claiming experience with less frequency may result in risking the situation even more. }\end{array}$} \\
\cline { 2 - 4 } & $\begin{array}{l}\text { Experience } \\
\text { Frequency }\end{array}$ & $\begin{array}{l}\text { It refers to some individuals' tendency to over-rely on experts, which, in other words, abandon or weaken their } \\
\text { responsibilities for monitoring and contributing to the situation's safety. This, in fact, weakens the process of } \\
\text { deference to expertise and breeds malfunction in the process of organizational reliability. }\end{array}$ \\
\hline
\end{tabular}




\section{Appendix D: Codes to Themes Development Schemes}

Table 25: Grouping and Converting Codes into Themes (PWF)

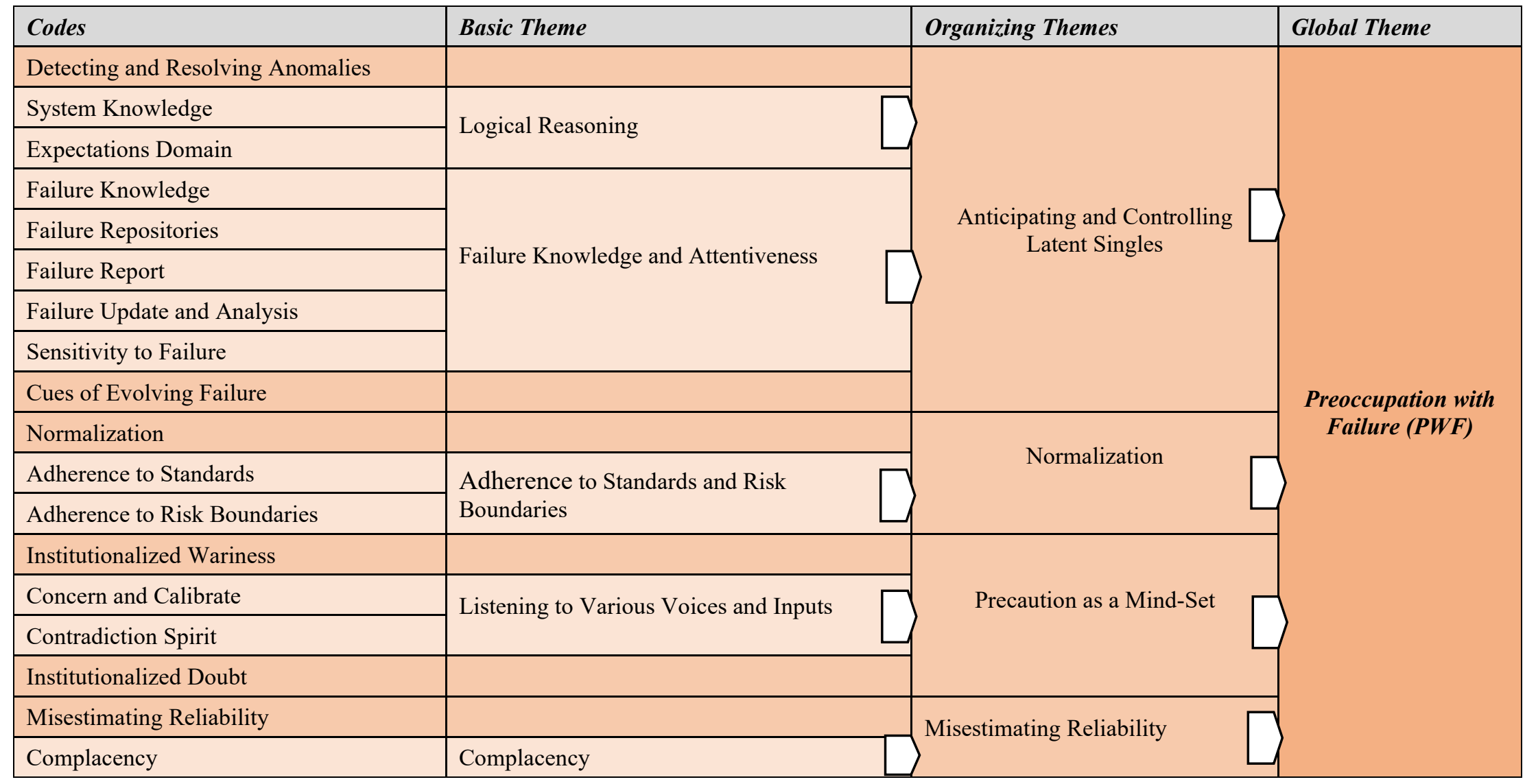


Table 26: Grouping and Converting Codes into Themes (RTS)

\begin{tabular}{|c|c|c|c|}
\hline Codes & Basic Theme & Organizing Themes & $\begin{array}{l}\text { Global } \\
\text { Theme }\end{array}$ \\
\hline Simplification & & \multirow{4}{*}{$\begin{array}{l}\text { Obscuring and Neglecting Significant } \\
\text { Information }\end{array}$} & \multirow{17}{*}{$\begin{array}{c}\text { Relectance } \\
\text { To Smplify } \\
\quad \text { (RTS) }\end{array}$} \\
\hline Generalization & & & \\
\hline Misidentification & & & \\
\hline Distinctions & Distinctions & & \\
\hline Organizing for Variety & & \multirow{7}{*}{ Organizing for Variety } & \\
\hline Requisite Variety & \multirow{6}{*}{ Requisite Variety and Complexity } & & \\
\hline Organizational Complexity & & & \\
\hline Complicated System & & & \\
\hline Complex System & & & \\
\hline Compilation System & & & \\
\hline Ad hoc Networks & & & \\
\hline Organizing for Sense-Discrediting & & \multirow{2}{*}{ Organizing for Sense-Discrediting } & \\
\hline Redundancy & Redundancy & & \\
\hline Organizing for Action-Based Inquiry & & \multirow{4}{*}{ Organizing for Action-Based Inquiry } & \\
\hline Actions Interpretation & \multirow{3}{*}{$\begin{array}{l}\text { Action Interpretation and Recursive } \\
\text { Interaction }\end{array}$} & & \\
\hline $\begin{array}{l}\text { Continual Reassessment of } \\
\text { Diagnoses }\end{array}$ & & & \\
\hline $\begin{array}{l}\text { Cultivation of Alternatives } \\
\text { Diagnoses }\end{array}$ & & & \\
\hline
\end{tabular}


Table 27: Grouping and Converting Codes into Themes (STO)

\begin{tabular}{|c|c|c|c|}
\hline Codes & Basic Theme & Organizing Themes & Global Theme \\
\hline \multicolumn{4}{|l|}{ Anchoring in the Present } \\
\hline Ignorance & \multirow{3}{*}{ Deviance of Attention } & \multirow{3}{*}{ Anchoring in the Present } & \multirow{19}{*}{$\begin{array}{c}\text { Sensitivity To } \\
\text { Operations (STO) }\end{array}$} \\
\hline Casualness & & & \\
\hline Distraction & & & \\
\hline Sustaining the Cognitive Map & & \multirow{7}{*}{$\begin{array}{c}\text { Operation as an Integrated } \\
\text { Map }\end{array}$} & \\
\hline Building Plausible Stories & \multirow{4}{*}{$\begin{array}{l}\text { Sustaining Dynamic Situational } \\
\text { Awareness }\end{array}$} & & \\
\hline Maintaining Real-time Awareness & & & \\
\hline Assessment and Continual Updates & & & \\
\hline Procedure Knowledge & & & \\
\hline Cognitive Limitations & \multirow{2}{*}{ Loss of Situational Awareness } & & \\
\hline Situational Awareness Drifts & & & \\
\hline Pressure & & Pressure & \\
\hline \multicolumn{2}{|l|}{ Heedful Interrelating } & \multirow{5}{*}{ Heedful Interrelating } & \\
\hline Contribution & \multirow{3}{*}{ Level of Employee Participation } & & \\
\hline Representation & & & \\
\hline \multirow{2}{*}{\multicolumn{2}{|c|}{$\begin{array}{l}\text { Subordination } \\
\text { Self-Interest }\end{array}$}} & & \\
\hline & & & \\
\hline \multicolumn{2}{|l|}{ Dysfunctional Momentum } & \multirow{3}{*}{ Dysfunctional Momentum } & \\
\hline Interruptions & \multirow[t]{2}{*}{ Interruptions and Adjustments } & & \\
\hline Adjustments & & & \\
\hline
\end{tabular}


Table 28: Grouping and Converting Codes into Themes (CTR)

\begin{tabular}{|c|c|c|c|}
\hline Codes & Basic Theme & Organizing Themes & Global Theme \\
\hline \multicolumn{4}{|l|}{ System Flexibility } \\
\hline Elasticity & \multirow[t]{2}{*}{ Elasticity and Recovery } & \multirow{3}{*}{$\begin{array}{l}\text { System Flexibility and } \\
\text { Adaptability }\end{array}$} & \multirow{13}{*}{$\begin{array}{l}\text { Commitment To } \\
\text { Resilience (CTR) }\end{array}$} \\
\hline Recovery & & & \\
\hline Resistance to Change & & & \\
\hline Improvisation & & \multirow{5}{*}{ Improvisation } & \\
\hline Simple Structure & Simple Structure & & \\
\hline General Knowledge & \multirow{3}{*}{ Personal Competence and Confidence } & & \\
\hline Interpersonal Skills & & & \\
\hline Confidence & & & \\
\hline Sensemaking & & \multirow[t]{2}{*}{ Sensemaking } & \\
\hline Reconstituting the Evolving Present & Recalibrating the Ongoing Present & & \\
\hline \multicolumn{2}{|l|}{ Responses (Actions) Repertoire } & \multirow{3}{*}{ Responses (Actions) Repertoire } & \\
\hline Learning from Failure/Incidents & \multirow[t]{2}{*}{ Learning from Failure } & & \\
\hline Negative Feedback & & & \\
\hline
\end{tabular}


Table 29: Grouping and Converting Codes into Themes (DTE)

\begin{tabular}{|c|c|c|c|}
\hline Codes & Basic Theme & Organizing Themes & Global Theme \\
\hline Structure Flexibility & & Structure Flexibility & \multirow{12}{*}{$\begin{array}{c}\text { Deference to } \\
\text { Expertise (DTE) }\end{array}$} \\
\hline Respectful Yielding & & \multirow{2}{*}{ Respectful Yielding } & \\
\hline Self-Awareness & Self-Awareness & & \\
\hline \multicolumn{2}{|l|}{ Relative Expertise } & \multirow{4}{*}{ Available Expertise } & \\
\hline Self-Importance & \multirow{3}{*}{ Ignoring Expertise } & & \\
\hline Equating Expertise to Experience & & & \\
\hline Equating Expertise to Authority & & & \\
\hline Domain-Specific Knowledge & & & \\
\hline Experience & & Domain-Specific Experience & \\
\hline Experience Depth & \multirow{2}{*}{ Compressed Experience } & & \\
\hline Experience Frequency & & & \\
\hline Reliance on Experts & & Reliance on Experts & \\
\hline
\end{tabular}




\section{Appendix E: Thematic Structures and Networks}

Table 30: Thematic Structure of RTS

\begin{tabular}{|c|c|c|c|}
\hline \multicolumn{4}{|c|}{ Reluctance to Simplify (RTS) - Global Theme (General Target) } \\
\hline & Distinctions (+) & $\rightarrow$ & Obscuring and Neglecting Significant Information (-) \\
\hline \multirow[t]{2}{*}{ 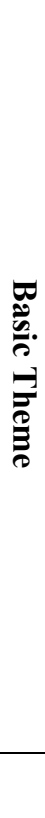 } & $\begin{array}{l}\text { Distinctions refer to differentiating things based on their specific } \\
\text { context and the details that they are providing. Distinctions are } \\
\text { significant in reducing simplifications, generalizations, and } \\
\text { misidentification through identifying more differences and to create } \\
\text { a richer and more varied picture of possible outcomes. This can then } \\
\text { recommend a deeper and more diverse set of precautions and early } \\
\text { warning indications. }\end{array}$ & 窟: & $\begin{array}{l}\text { This is a product of actions-based tendencies to simplify, generalize, } \\
\text { and often misidentify significant information. People tend to simplify } \\
\text { the context that they work within to better understand it. However, } \\
\text { simplification and generalization cause people to miss, neglect, or } \\
\text { obscure unwanted, unanticipated, unexplainable important details, } \\
\text { increasing the probability of producing an unreliable outcome. Also, } \\
\text { simplification reduces people the cognitive capacity to comprehend } \\
\text { complexity, which puts them in tough situations when the unexpected } \\
\text { occurs. Furthermore, obscuring and neglecting significant information } \\
\text { happens when interpreting and treating information based on a general } \\
\text { description. Generalization occurs when people categorize their world. } \\
\text { Thus, they interpret what they notice or perceive based on what they } \\
\text { already know or have known. These types of categorizations glide over } \\
\text { fine-grained distinctions that may predict the unexpected. As a result, } \\
\text { people misidentify an issue based on the identification factors or } \\
\text { symptoms of other problems that have already been identified. }\end{array}$ \\
\hline & Requisite Variety and Complexity (+) & $\rightarrow$ & Organizational Variety (+) \\
\hline
\end{tabular}




\begin{tabular}{|c|c|c|c|}
\hline \multirow[t]{2}{*}{ 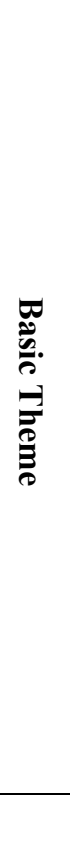 } & $\begin{array}{l}\text { Requisite variety or requisite complexity refers to diversifying the } \\
\text { perspectives among the organization members over the traditional } \\
\text { means to achieve reliability in a complex environment. For example, } \\
\text { experiments showed that a task managed by people from varied } \\
\text { functional, educational, experiential backgrounds is most likely to be } \\
\text { successful than if people of similar backgrounds managed it. Since } \\
\text { HROs design requisite variety to ensure various inputs, they } \\
\text { fabricate complexity in order to allow disagreements and conflicts as } \\
\text { normal interactions. This widens the range of comprehending } \\
\text { complexity inherent in such organizations. However, conflicts must } \\
\text { be negotiated, managed, and controlled. These efforts take such } \\
\text { forms as diverse checks and balances embedded in the circulation of } \\
\text { committees and meetings, frequent adversarial reviews, selecting } \\
\text { new employees with non-typical prior experience, regular job } \\
\text { rotation, and re-training. Designing requisite variety is an attribute in } \\
\text { the HROs to leverage and sustain the organization's goal in } \\
\text { organizing for variety. }\end{array}$ & 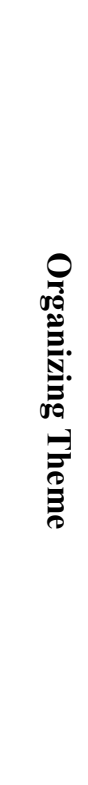 & $\begin{array}{l}\text { Organizational variety is the organizational design that increases the } \\
\text { process base of sensing, analyzing, and responding to various inputs. } \\
\text { In a complex system, complexity is the only way to cope with the } \\
\text { complex environment and sustain healthy conditions. It has been } \\
\text { acknowledged that diverse groups have a more comprehensive range } \\
\text { of information than those in homogeneous groups. Organizing for } \\
\text { variety can be achieved and sustained through designing requisite } \\
\text { variety and organizational complexity. However, organizations should } \\
\text { be careful when they design the system structure., For instance, } \\
\text { complicated systems are considered undesirable since they seek } \\
\text { simplifications resulting from developing a common operational } \\
\text { image among individuals. Therefore, it has been suggested that two } \\
\text { types of systems design can achieve the desired complexity; a complex } \\
\text { system that is adapted for requisite variety and to create distinctions by } \\
\text { the interactions among the constituents; and compilation systems, } \\
\text { which develop emergent complexity in addition to the use of the } \\
\text { existing one, such as the ad-hoc networks. }\end{array}$ \\
\hline & Redundancy (+) & $\rightarrow$ & Organizing for Sense-Discrediting $(+)$ \\
\hline 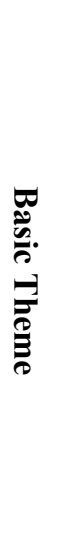 & $\begin{array}{l}\text { Redundancy is defined as a designed form of duplications and } \\
\text { backups in the system. It is a backup for technical, individuals, and } \\
\text { organizational elements. Redundancy takes a form of skepticism, } \\
\text { which involves cross-checks, doubts that precautions are sufficient, } \\
\text { and wariness about claimed levels of competence. Redundancy can } \\
\text { make the system more complex, resulting in eliminating } \\
\text { oversimplifications and misidentifications by making the system } \\
\text { denser and varied. However, redundancy may encourage } \\
\text { complacency since the system's frontline elements can overly be } \\
\text { relayed on the second line elements in fixing errors and assuring that } \\
\text { everything is processed smoothly. }\end{array}$ & 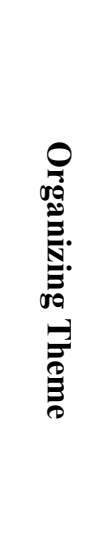 & $\begin{array}{l}\text { This refers to embedding uncertainties into existing concepts that } \\
\text { confront dynamic, complex environments, thereby organizing for } \\
\text { more openness. In other words, the system is open to new information } \\
\text { and new experiences and, therefore, can cope with such environments. } \\
\text { Systems confronted with the dynamic complex environment need to } \\
\text { be able to develop intelligent ideas about that environment (sense- } \\
\text { making), but at the same time, need to be able to criticize their ideas } \\
\text { (sense-discrediting). Therefore, organizing for sense-discrediting } \\
\text { enables the organization to slow simplifications and allow for } \\
\text { simultaneous change and stability. Thus, sense-discrediting functions } \\
\text { act as a balancing force. To achieve this, HROs design redundancies } \\
\text { as a backup and double-checks for system elements. }\end{array}$ \\
\hline
\end{tabular}




\begin{tabular}{|c|c|c|c|}
\hline & Action Interpretation and Recursive Interaction (+) & $\rightarrow$ & Organizing for Action-Based Inquiry $(+)$ \\
\hline & $\begin{array}{l}\text { This pattern works as a source for action based-inquiry where action } \\
\text { interpretation provides more comprehensible information extracted } \\
\text { from the actions of those who solve problems and interpret actions } \\
\text { for learning purposes. Problem solvers continue to reassess the } \\
\text { diagnoses by conducting frequent examinations and evaluations of } \\
\text { the available diagnoses' credibility. This process continues until } \\
\text { these diagnoses make sense. If the diagnoses do not make sense, they } \\
\text { keep looking for more options to reduces the vulnerability of } \\
\text { producing misjudgments. As they continue with the process, they } \\
\text { collect more possibilities by expanding the boundaries to consider a } \\
\text { broader set of alternatives, enhancing the anticipation and flexibility } \\
\text { needed for mindful organizing. }\end{array}$ & 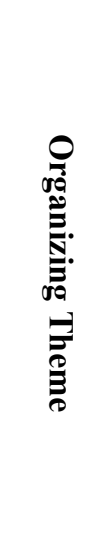 & $\begin{array}{l}\text { This refers to establishing a pattern for generating more information } \\
\text { from actions, particularly when people confront the unexpected, which } \\
\text { means that the information cues become available only when actions } \\
\text { are taken, or in other words, action informs systems. This pattern } \\
\text { precludes the tendencies to simplify the already simplified information } \\
\text { further. This encourages the search for more details, creating questions } \\
\text { for broader explanations, and allows for more distinctions. To achieve } \\
\text { this pattern, a sequence of interpreted actions is required, followed by } \\
\text { a continual reassessment of the available diagnosis, and the cultivation } \\
\text { of alternatives should be ongoing involving what is described as } \\
\text { "recursive interactions between interpreting and updating." }\end{array}$ \\
\hline
\end{tabular}




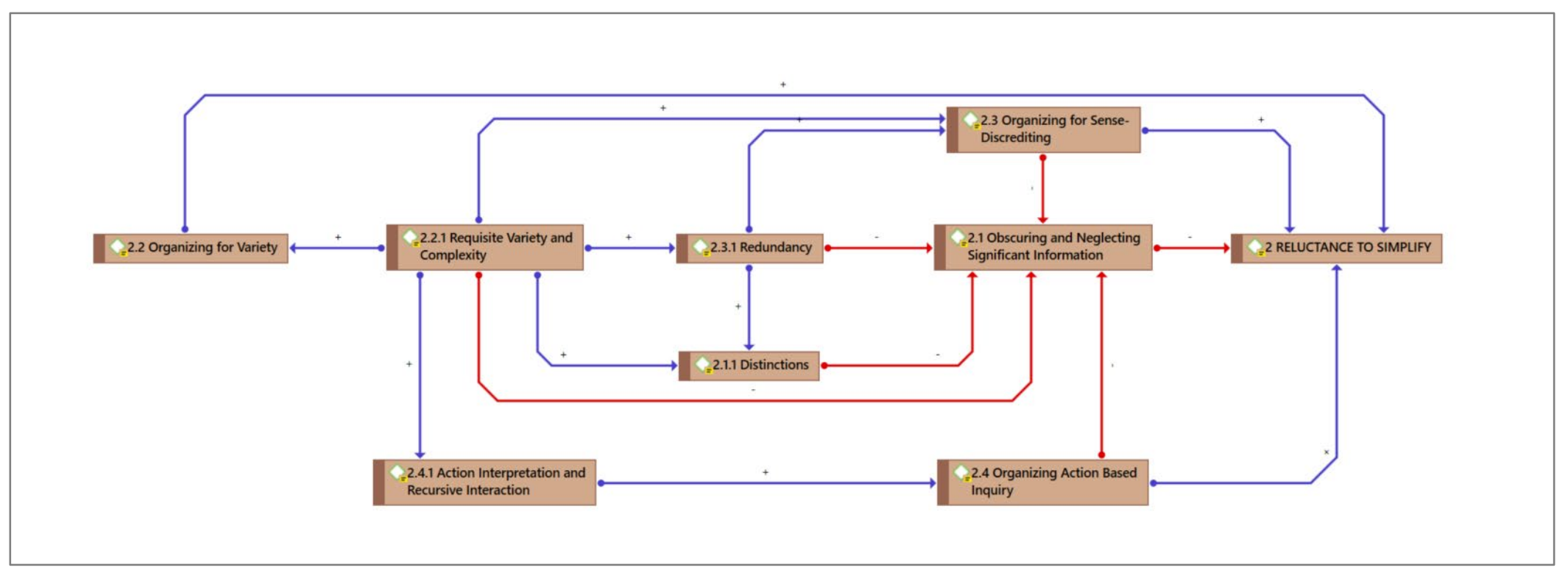

Figure 48: Thematic Network of RTS: Developed with Atlas.ti 
Table 31: Thematic Structure of STO

\begin{tabular}{|c|c|c|c|}
\hline \multicolumn{4}{|c|}{ Sensitivity to Operations (STO) - Global Theme (General Target) } \\
\hline & Loss of Attention (-) & $\rightarrow$ & Staying Focused (+) \\
\hline \multirow[t]{2}{*}{ 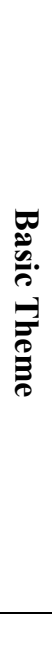 } & $\begin{array}{l}\text { Loss of attention is a product of three states of mind that adversely } \\
\text { deviate the attention and mental concentration on operations: } \\
\text { ignorance, casualness, and distractions. Ignorance refers to the } \\
\text { intentional or unintentional neglect of safe work procedures. This } \\
\text { could be a result of self-complacency or personal culture. } \\
\text { Casualness is a product of people's tendency to simplify situations } \\
\text { and issues around the context they are working within. Casualness } \\
\text { provides superficial interpretations when it is essential to interpret } \\
\text { issues (e.g., signals and alarms) on a deeper level to diagnose what } \\
\text { is happening and take appropriate action. However, distraction } \\
\text { refers to whatever reduces concentration and focus from work in the } \\
\text { present moment. Loss of attention could result from the work itself, } \\
\text { such as fatigue. }\end{array}$ & 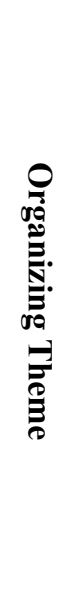 & $\begin{array}{l}\text { Focus is defined as continual concentration and attention on the task at } \\
\text { hand. Staying focused on the present increases attentiveness and } \\
\text { readiness for quick responses. However, loss of or wavering attention } \\
\text { could lead to lapses that eventually cause operators to lose control and } \\
\text { preclude their capacity to detect and resolve anomalies and cues of } \\
\text { evolving failures. Staying focused in the present requires a clear mind, } \\
\text { acting while thinking, thinking by acting, and thinking through acting. }\end{array}$ \\
\hline & Sustaining Dynamic Situational Awareness (+) & $\rightarrow$ & Operations as an Integrated Map (+) \\
\hline
\end{tabular}




\begin{tabular}{|c|c|c|c|}
\hline 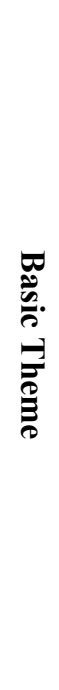 & $\begin{array}{l}\text { Sustaining dynamic situational awareness means to construct and } \\
\text { continuously maintain the cognitive map. Operators need to build } \\
\text { stories to process complex inputs, references, explanations, and } \\
\text { definitions of the total situation. For example, operators examine the } \\
\text { described actions in the procedure steps to see if they make sense in } \\
\text { the context of a particular event. However, to build such stories, } \\
\text { operators are required to maintain real-time situational awareness, } \\
\text { by which they remain mindful and knowledgeable of the elements } \\
\text { of their work environment. Sustaining dynamic situational } \\
\text { awareness during real-time operations can be achieved by reducing } \\
\text { or mitigating any laps between the perceptions and the ongoing } \\
\text { operations. It requires both assessment and continual updates. This } \\
\text { refers to the constant examination of any new emergent inputs, their } \\
\text { interpretations, and definitions, by which operators continue } \\
\text { assessing their situational awareness. }\end{array}$ & & $\begin{array}{l}\text { This refers to the construction and maintenance of the cognitive map, } \\
\text { allowing operators to integrate diverse inputs, information sensors and } \\
\text { remote observations, and the real-time status and performance. This is } \\
\text { called "having the bubble" in US Navy ships. This "bubble" is } \\
\text { considered necessary, particularly in coping with any work surprises } \\
\text { that might arise in automated operations. However, to conceptualize the } \\
\text { operations as an integrated map, operators and involved personnel need } \\
\text { to sustain a dynamic situational awareness through building stories, } \\
\text { maintaining real-time situational awareness, conducting assessments, } \\
\text { and continually generating updates. For operators to do so, they need to } \\
\text { have a working understanding of the operations and procedure } \\
\text { knowledge, which is defined as the logic of the series of actions } \\
\text { conducted in a certain order or manner to execute operations. Operators } \\
\text { use knowledge of the assumptions and reasoning that underlie } \\
\text { preplanned procedures to deal with situations not covered by procedure. }\end{array}$ \\
\hline & Loss of Situational Awareness (-) & $\rightarrow$ & Operations as an Integrated Map (+) \\
\hline 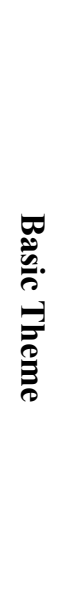 & $\begin{array}{l}\text { Situational awareness is lost due to cognitive limitations and drifts. } \\
\text { Individual cognition is the mental resources to process and integrate } \\
\text { the cognitive map through building stories and maintaining real- } \\
\text { time awareness and assessment. However, an individual's cognitive } \\
\text { capacity is naturally limited and is possibly reduced even more when } \\
\text { they experience stress, fatigue, distraction, and other job-related } \\
\text { effects. When ongoing operations do not fully occupy cognitive } \\
\text { capacity, maintaining situational awareness in real-time becomes an } \\
\text { issue, and laps occur, which endangers a reliable outcome. } \\
\text { Moreover, when situational awareness drifts, operators fail to notice } \\
\text { the error and to detect anomalies. This failure can lead to inaccurate } \\
\text { simplifications, misinterpreted intentions and plans, reliance on an } \\
\text { established routine, and following authorities blindly. }\end{array}$ & 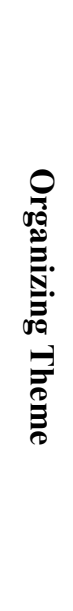 & \\
\hline
\end{tabular}




\begin{tabular}{|c|c|c|c|}
\hline & & $\rightarrow$ & Pressure (-) \\
\hline 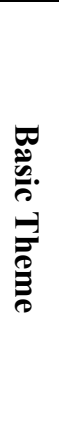 & & 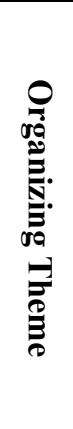 & $\begin{array}{l}\text { Pressure refers to any work burdens, particularly production pressure, } \\
\text { which often happens in organizations where production is the } \\
\text { conclusive success factor. Pressure reduces a worker's focus on the task } \\
\text { at hand, causes them to be distracted, weakens their ability to judge and } \\
\text { interpret correctly, and accurately. It also causes them to simplify, } \\
\text { generalize, and misidentify the received and processed information, } \\
\text { which causes them to fall back on first-learned reactions. This } \\
\text { eventually leads to severe consequences, whereby operators lose their } \\
\text { sensitivity to operations. }\end{array}$ \\
\hline & Level of Employee Participation (+) & $\rightarrow$ & Heedful Interrelating (+) \\
\hline 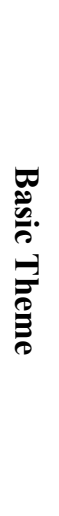 & $\begin{array}{l}\text { This is a pattern of three practices: contribution, representation, and } \\
\text { subordination that develop a social process by which one can } \\
\text { perceive the relationships among the organization's members. } \\
\text { Contribution points to the involvement of the organization's } \\
\text { members in creating activities and functions that serve the long-term } \\
\text { goal. Representation refers to how the organization members view } \\
\text { or imagine their contributions to the process, operations, and general } \\
\text { success or failure of the goal. Subordination refers to the condition } \\
\text { where the organization members are subordinate to the } \\
\text { organization's dominant culture and system by asking what it needs } \\
\text { and acting in ways intended to meet those needs. }\end{array}$ & 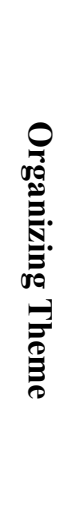 & $\begin{array}{l}\text { Heedful interrelating refers to a collective mind or social process where } \\
\text { individual actions are part of a larger shared action pattern. They } \\
\text { understand how their efforts fit into that more extensive pattern. This } \\
\text { level of heedfulness explains how operations are tied together by the } \\
\text { patterns shaping the level of the members' participation in the } \\
\text { organization's total outcome, including performance, safety, success, } \\
\text { or failures. Furthermore, these patterns are implemented with care, } \\
\text { alertness, and wariness during real-time operations. }\end{array}$ \\
\hline & Self-Interest (-) & $\rightarrow$ & Heedful Interrelating (+) \\
\hline & $\begin{array}{l}\text { Self-interest is when members of the organization view their jobs } \\
\text { based on their interests and divide the functions based on their roles } \\
\text { and responsibilities. This type of context works against } \\
\text { subordination particularly, and the heedful interrelating generally. }\end{array}$ & & \\
\hline
\end{tabular}




\begin{tabular}{|l|l|l|l|}
\hline Interruptions and Adjustments (+) & $\rightarrow$ & Dysfunctional Momentum (-) \\
\hline $\begin{array}{l}\text { It is the pattern of actions where dysfunctional momentum can be } \\
\text { managed, and its consequences can be mitigated, particularly in } \\
\text { situations and contexts where the operations are failing. }\end{array}$ & $\begin{array}{l}\text { Dysfunctional momentum is keeping to the course of action without } \\
\text { reassessing the total situation, particularly when the operations suffer } \\
\text { lapses and unnoticed cues. It is a result of routinely recurring events } \\
\text { during operations that when they fail, they continue to fail until a } \\
\text { trigger sensemaking as it is a form of sensitivity to operations. } \\
\text { Interruptions require rethinking and adjustments, which refer to the } \\
\text { opportunities to preclude mistakes and errors from lining up so that } \\
\text { they grow into more significant issues. This includes deploying } \\
\text { resources whenever and wherever needed. }\end{array}$ & $\begin{array}{l}\text { Dysfunctional momentum is a clear indication that people are not } \\
\text { sensitive to the operations and that the organization is preoccupied with } \\
\text { efficiency over reliability. }\end{array}$ \\
\hline
\end{tabular}




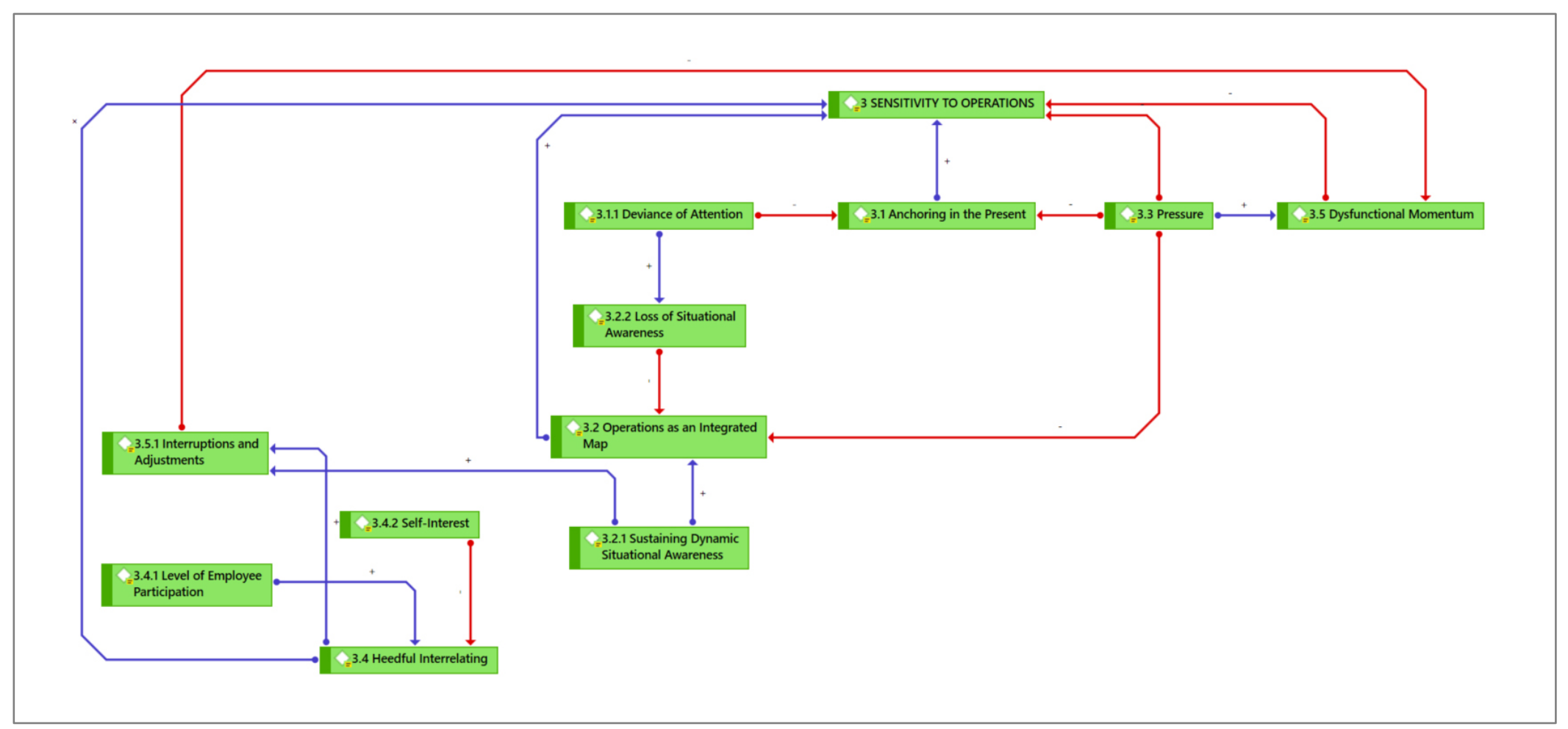

Figure 49: Thematic Network of STO: Developed with Atlas.ti 
Table 32: Thematic Structure of CTR

\begin{tabular}{|c|c|c|c|}
\hline \multicolumn{4}{|c|}{ Commitment to Resilience (CTR) - Global Theme (General Target) } \\
\hline & Elasticity and Recovery $(+)$ & $\rightarrow$ & System Flexibility and Adaptability (+) \\
\hline \multirow[t]{2}{*}{ 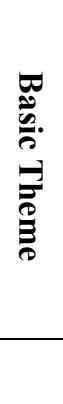 } & $\begin{array}{l}\text { Elasticity and recovery refer to the organizational ability to expand } \\
\text { and bounce back, hence being both elastic and recoverable. Elasticity } \\
\text { refers to the capacity of stretching without breaking, or in other } \\
\text { words, expanding enough when it is required to contain any threats. } \\
\text { Recovery means to return, either slowly or quickly, into some form, } \\
\text { so that the organization, whenever faced with unexpected events, can } \\
\text { presume its activities. }\end{array}$ & 产 & $\begin{array}{l}\text { For a system to be elastic and recoverable, or in other words, to expand } \\
\text { and bounce back, the system should be flexible enough without causing } \\
\text { any damage. This term concerning HROs means that both individuals } \\
\text { and the organization should be flexible enough to cope with the } \\
\text { unexpected and resume normal operations. System adaptability refers } \\
\text { to maintaining the same outputs or effects even when the conditions of } \\
\text { the operations are varying. }\end{array}$ \\
\hline & Simple Structure (+) & $\rightarrow$ & Improvisation $(+)$ \\
\hline 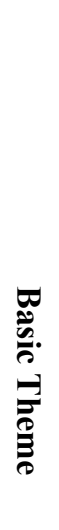 & $\begin{array}{l}\text { Simple structure refers to the preexisting or minimal structure that } \\
\text { provides experienced people with a framework to act (improvise) } \\
\text { within. This simple structure could be a set of core values, } \\
\text { regulations, rules of engagement, or the mission statement, within } \\
\text { which people can direct themselves and engage with sensemaking. }\end{array}$ & 产 & $\begin{array}{l}\text { Improvisation refers to the ability to act upon emergent threats, } \\
\text { particularly when those involved encounter lack of resources, such as } \\
\text { no information regarding the experienced situation. Improvisation } \\
\text { involves rearranging and examining the intuitive understanding of the } \\
\text { experienced phenomena, while the ongoing action can still make a } \\
\text { difference. Improvisation should be able to see the details of the threa } \\
\text { even within the most complex environment. It requires a mix of skills, } \\
\text { knowledge, and intuitive recombination based on a simple structure that } \\
\text { allows for such actions. }\end{array}$ \\
\hline
\end{tabular}




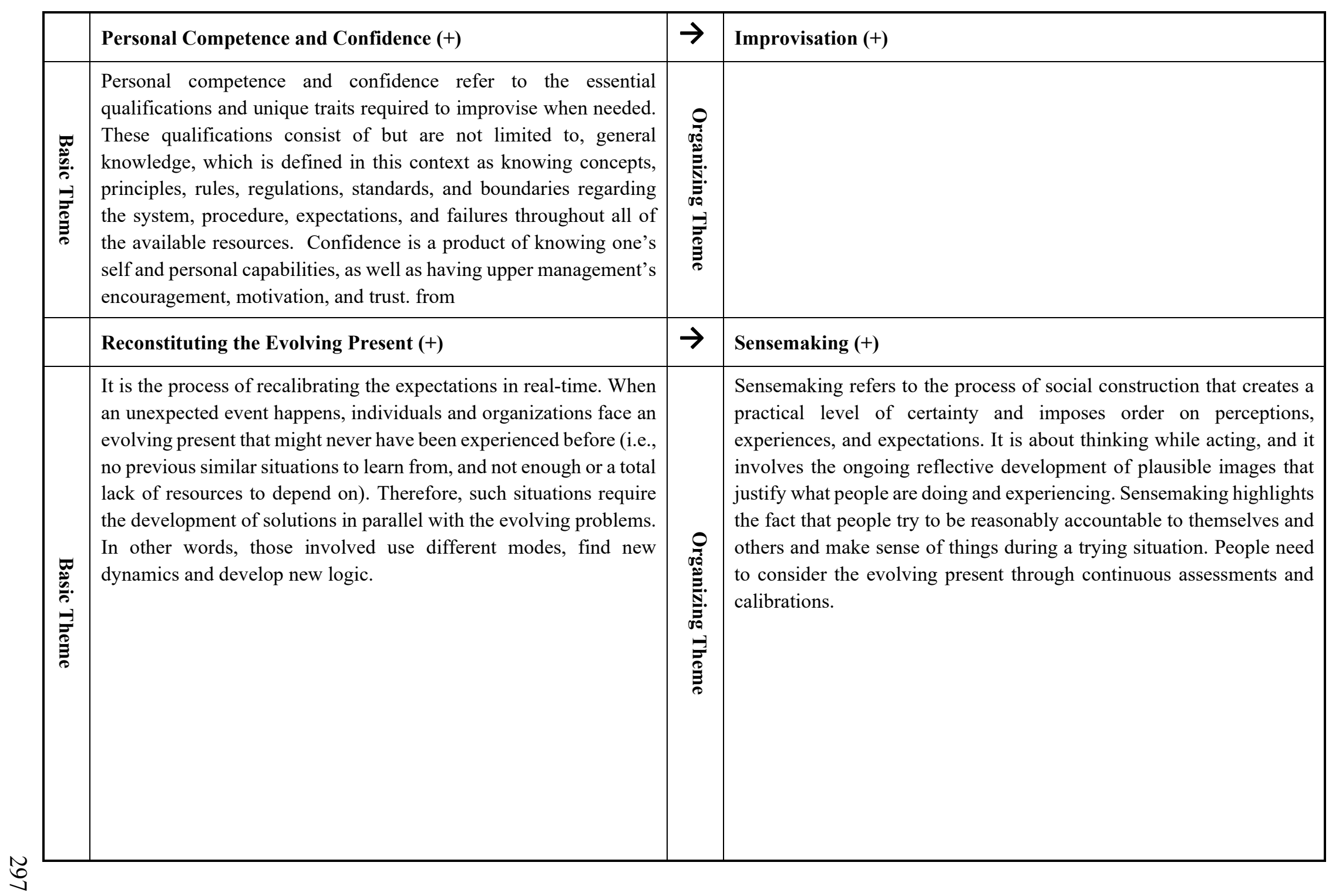




\begin{tabular}{|c|c|c|c|}
\hline & Learning from Failure/Incident $(+)$ & $\rightarrow$ & Responses (Actions) Repertoire (+) \\
\hline 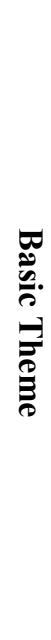 & $\begin{array}{l}\text { Learning from failure or incidents is a key factor to operate } \\
\text { resiliently. This theme refers to expanding the repertoire of the } \\
\text { information already generated from previous errors (failures) and } \\
\text { implementing that learning through fast and negative feedback. This } \\
\text { practice was recently labeled "Learning from incident LFI." It was } \\
\text { given a lot of attention from academia and practitioners since this } \\
\text { type of learning enlarges the response repertoire and positively } \\
\text { increases the capability of commitment to resilience. Learning from } \\
\text { failure requires negative feedback, which is fast-real-time learning } \\
\text { that allows people to cope with an unfolding surprise in ways that } \\
\text { are not specified in advance. It is at the base of the learning process } \\
\text { from failure, whereby operators tend to end or reduce the } \\
\text { fluctuations in the output failures or errors. It is sometimes called } \\
\text { double-loop learning. }\end{array}$ & 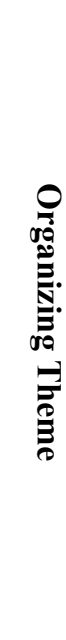 & $\begin{array}{l}\text { It is an extensive and rich reference for developing capabilities for } \\
\text { commitment to resilience. This is built through training and simulation, } \\
\text { varied job experiences, learning from negative feedback, and ad hoc } \\
\text { networks that allow for rapid pooling of expertise to handle } \\
\text { unanticipated events. Responses or action repertoire include different } \\
\text { levels of information richness, collaborative practice, and pro-active } \\
\text { focus. Organizational knowledge within HROs should be described by } \\
\text { a wide-ranging action repertoire to redefine investigation and learning } \\
\text { by trial-and-error and a high degree of pro-active focus to prepare for } \\
\text { unexpected events. }\end{array}$ \\
\hline
\end{tabular}




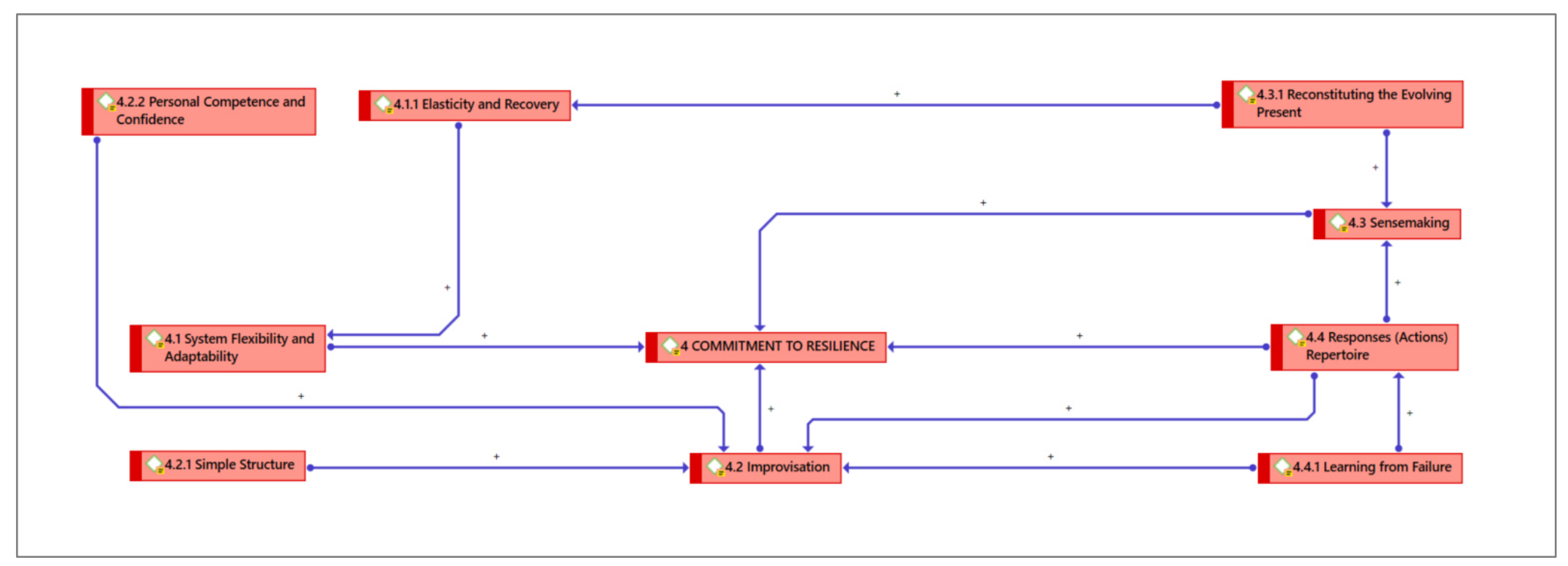

Figure 50: Thematic Network of CTR: Developed with Atlas.ti 
Table 33: Thematic Structure of DTE

\begin{tabular}{|c|c|c|c|}
\hline \multicolumn{4}{|c|}{ Deference to Expertise (DTE) - Global Theme (General Target) } \\
\hline & & $\rightarrow$ & Structure Flexibility (+) \\
\hline \multirow[t]{2}{*}{ 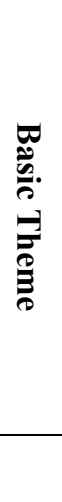 } & & : & $\begin{array}{l}\text { Structural flexibility loosens or unties the hierarchical structure's } \\
\text { rigidity to allow for the decision-making process to migrate to lower } \\
\text { levels, particularly to those on frontlines during situations of } \\
\text { emergencies and serious threats. In a reliable or mindful system, the } \\
\text { structure is a variable, but structuring is constant. This property requires } \\
\text { collective cultural beliefs so that the necessary competence lies } \\
\text { somewhere in the system and that migrating problems will find them. } \\
\text { These beliefs are associated with a more mindful recruitment of } \\
\text { expertise. }\end{array}$ \\
\hline & Self-Awareness (+) & $\rightarrow$ & Respectful Yielding (+) \\
\hline 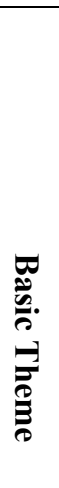 & $\begin{array}{l}\text { Self-awareness refers to knowing and acknowledging the } \\
\text { knowledge and experiences of the self, as well as the limits and gaps } \\
\text { in them. Therefore, a true expert does not hesitate to inquire about } \\
\text { others' knowledge when he/she needs it. Besides, this personal } \\
\text { attribute guides the individuals to yield respectfully to other } \\
\text { individuals with a more profound level of expertise and experience. }\end{array}$ & 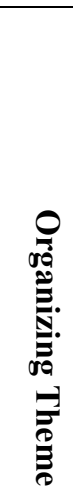 & $\begin{array}{l}\text { Respectful yielding is the interactions or subordination among the } \\
\text { members of the organization. It happens relative to the performance } \\
\text { criteria applied in a particular context. In other words, it interprets an } \\
\text { action, answers a question, solves a problem, provides advice, and so } \\
\text { on. Respectful yielding as a property of deference to expertise reflects } \\
\text { its nature as relational rather than an individual. It is considered the } \\
\text { foundation of shared understanding and communication. }\end{array}$ \\
\hline
\end{tabular}




\begin{tabular}{|c|c|c|c|}
\hline & & $\rightarrow$ & Available Expertise (+) \\
\hline \multirow[t]{2}{*}{ 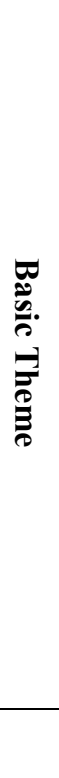 } & & 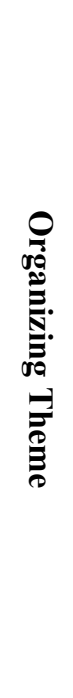 & $\begin{array}{l}\text { Expertise is a co-production of modified, varied, and sometimes } \\
\text { conflicted opinions, supplying data sources, and mixed experiences and } \\
\text { additional inputs to discussions and conversations. Experts can make } \\
\text { sense regarding a subject or an issue because they pay close attention } \\
\text { to context. Expertise is known to solve problems or answer questions } \\
\text { relating to a specific problem-solving domain or area of expertise. In } \\
\text { HROs, those experts who are termed as reliability professional "RPs," } \\
\text { are generally part of teams, have special perspectives on reliability, both } \\
\text { cognitively and normatively. They have been termed "reliability } \\
\text { professionals," although they need not be holders of particular } \\
\text { positions, have professional degrees, or even any higher degrees at all. } \\
\text { They can be found at all levels of HROs - some are top-level } \\
\text { executives. Still, they can also be found among department heads, } \\
\text { program managers, shift supervisors, and on down to control operators, } \\
\text { engineers, and technicians. }\end{array}$ \\
\hline & Compressed Experience (+) & $\rightarrow$ & Domain-Specific Experience (+) \\
\hline 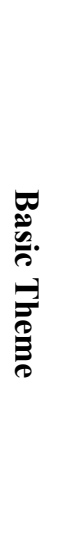 & $\begin{array}{l}\text { Compressed experience clarifies expertise and defines the core of } \\
\text { the expert's role. In other words, an expert who spent a certain } \\
\text { period and made efforts in a specific domain knows better than those } \\
\text { whose experiences are in a different domain. However, knowledge } \\
\text { in this context is attainable when people are willing to spend more } \\
\text { time and effort to gain the necessary experience. Compressed } \\
\text { experience is, therefore, an experience-based knowledge that results } \\
\text { from both depth and frequency, or in other words, frequent } \\
\text { encounters and handling with risks and stresses, as well as lessons } \\
\text { learned from closures of bad events. }\end{array}$ & 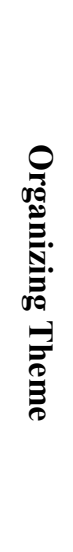 & $\begin{array}{l}\text { Domain-specific experience is the product of domain-specific } \\
\text { knowledge and generalized experience. Domain-specific knowledge is } \\
\text { defined as the personal, simulated, or virtual experience. This } \\
\text { knowledge is relevant to a specific domain that is a class of problems. } \\
\text { Much problem solving involves domain-specific knowledge. On the } \\
\text { other hand, generalized experience implies that what we have known } \\
\text { comprises some understanding of the nature and functioning of the } \\
\text { expertise we know to whom we defer. Besides, it is comprised of } \\
\text { experience with specific unexpected events. Firsthand experience in a } \\
\text { specific domain is more or less meaningful, depending on the frequency } \\
\text { and depth of that experience; or in other words, it requires compressed } \\
\text { experience. }\end{array}$ \\
\hline
\end{tabular}




\begin{tabular}{|c|c|c|}
\hline & $\rightarrow$ & Ignoring Expertise (-) \\
\hline \multirow[t]{3}{*}{ 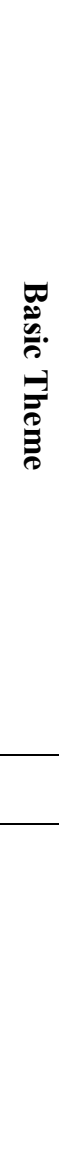 } & 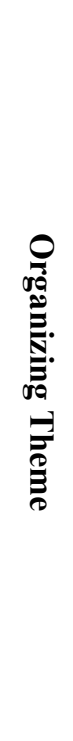 & $\begin{array}{l}\text { Expertise is minimized when it is neglected or ignored, such as when } \\
\text { organizations acquire other organizations. Thus, the newly parent } \\
\text { organizations do not give the transferred expertise the required attention } \\
\text { and importance. People consider expertise as products of years of } \\
\text { experience or a reflection of the higher rank and authorities. Therefore, } \\
\text { they equate them to their experience or authorities, which either } \\
\text { minimizes the true expertise or defers to the wrong ones. Another factor } \\
\text { that leads to minimizing expertise is self-importance, which refers to } \\
\text { falsely ranking the self-based on pursuing central positions or } \\
\text { conducting sensitive work due to the level of the risk inherent in the } \\
\text { job, which might be deceptively confirmed when people tell those with } \\
\text { self-importance that they are essential. However, such responsibilities } \\
\text { do not mean that these experts are all-knowing. The problem lies when } \\
\text { others mistakenly hide essential information, assuming that these so- } \\
\text { called experts already know it, while they may not. Therefore, in } \\
\text { solving problems, people respectfully defer to the wrong experts. }\end{array}$ \\
\hline & $\rightarrow$ & Overreliance on Experts (-) \\
\hline & 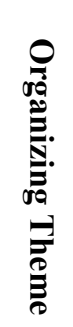 & $\begin{array}{l}\text { Overreliance on Experts refers to the tendency of some individuals to } \\
\text { overly depend on experts, which in other words abandon or weaken } \\
\text { their responsibilities for monitoring and contributing to the situation's } \\
\text { safety. This weakens the process of deference to expertise and breeds } \\
\text { malfunction in the process of organizational reliability. }\end{array}$ \\
\hline
\end{tabular}




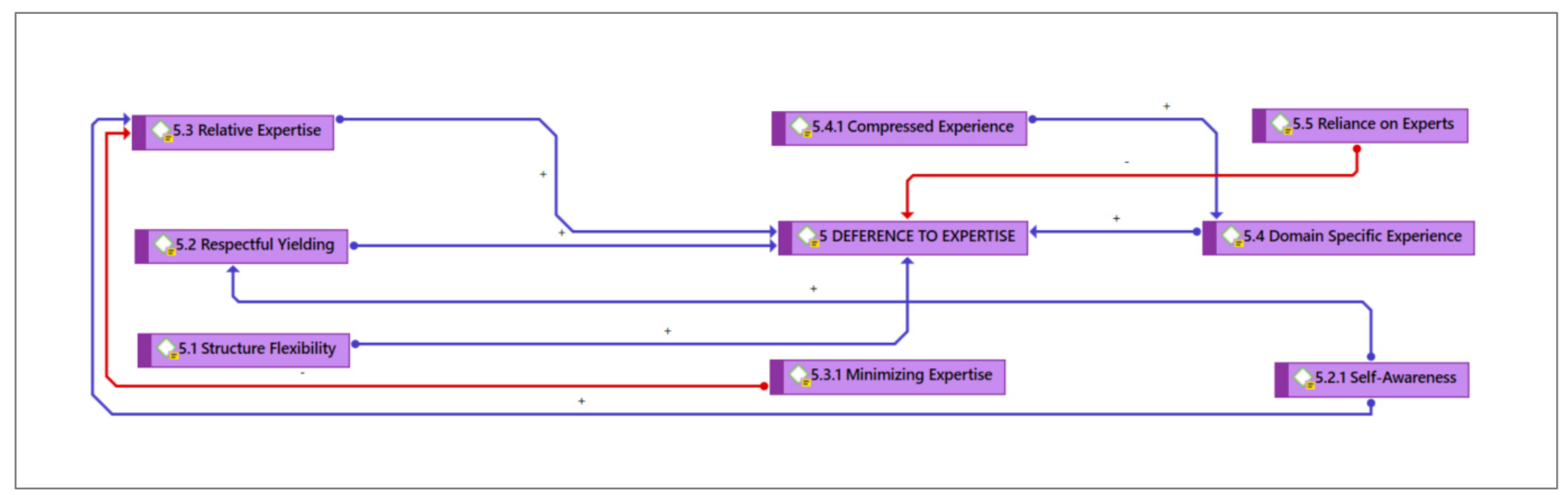

Figure 51: Thematic Network of DTE: Developed with Atlas.ti 


\section{Appendix F: FCM's Edges Weights Comparison Based on Three Measures}

Table 34: Edges Weights Comparison Based on Three Measures

\begin{tabular}{|c|c|c|c|c|c|c|c|c|c|c|c|c|c|c|c|}
\hline 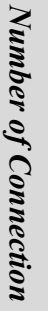 & Source & 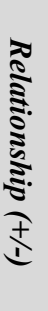 & Target & 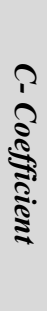 & 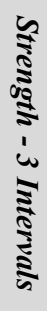 & 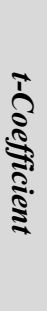 & 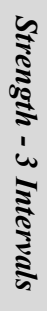 & 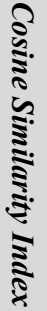 & 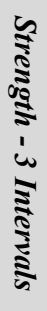 & 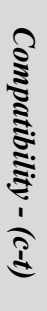 & 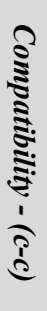 & 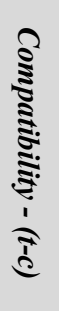 & 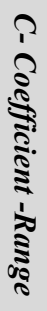 & 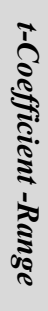 & 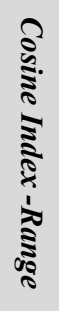 \\
\hline- & $\begin{array}{l}\text { Action Interpretation and Recursive } \\
\text { Interaction }\end{array}$ & + & Organizing Action Based Inquiry & $\stackrel{0}{\dot{0}}$ & 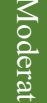 & $\stackrel{\circ}{\stackrel{\oplus}{\omega}}$ & 离 & in & 竞 & - & - & - & $\dot{a}$ & ㅇ. & ஜे \\
\hline N & $\begin{array}{l}\text { Action Interpretation and Recursive } \\
\text { Interaction }\end{array}$ & + & Distinctions & $\stackrel{\circ}{\dot{O}}$ & $\underset{8}{\substack{\infty \\
\frac{1}{\pi}}}$ & i & 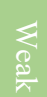 & $\underset{\text { in }}{\dot{w}}$ & $\frac{3}{8}$ & - & 0 & 0 & i & i & ஜे \\
\hline$\omega$ & $\begin{array}{l}\text { Adherence to Standards and Risk } \\
\text { Boundaries }\end{array}$ & - & Normalization & $\stackrel{\circ}{\circ}$ & $\begin{array}{l}\sum \\
\frac{8}{\pi}\end{array}$ & $\stackrel{0}{\ddot{\rho}}$ & $\begin{array}{l}3 \\
0 \\
0 \\
0 \\
0 \\
0\end{array}$ & $\stackrel{\circ}{\text { à }}$ & $\begin{array}{l}\overrightarrow{0} \\
\frac{0}{0} \\
\overrightarrow{0}\end{array}$ & 0 & 0 & - & $\underset{\omega}{\omega}$ & ஜे & $\begin{array}{l}\circ \\
\text { aे }\end{array}$ \\
\hline 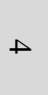 & $\begin{array}{l}\text { Adherence to Standards and Risk } \\
\text { Boundaries }\end{array}$ & + & $\begin{array}{l}\text { Anticipating and Controlling Latent } \\
\text { Singles }\end{array}$ & $\dot{\circ}$ & $\sum_{\substack{\pi \\
\frac{1}{2}}}^{\infty}$ & 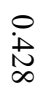 & 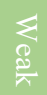 & $\underset{\sim}{\dot{u}}$ & $\frac{2}{0}$ & - & 0 & 0 & is & $\underset{\omega}{\text { i }}$ & 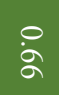 \\
\hline
\end{tabular}

$\stackrel{\infty}{\not}$ 


\begin{tabular}{|c|c|c|c|c|c|c|c|c|c|c|c|c|c|c|c|}
\hline$u$ & $\begin{array}{l}\text { Adherence to Standards and Risk } \\
\text { Boundaries }\end{array}$ & - & $\begin{array}{l}\text { Obscuring and Neglecting Significant } \\
\text { Information }\end{array}$ & $\stackrel{\circ}{\grave{N}}$ & $\sum_{\substack{2 \\
\frac{1}{2}}}^{\frac{2}{2}}$ & $\underset{0}{i \omega}$ & $\sum_{\substack{1 \\
\frac{1}{\pi}}}^{2}$ & $\underset{\substack{\infty \\
\infty}}{\substack{\infty \\
\infty}}$ & $\sum_{\substack{1 \\
\frac{\pi}{2}}}^{\frac{1}{2}}$ & - & - & 一 & i & i & $i_{\omega}$ \\
\hline$a$ & Anchoring in the Present & & SENSITIVITY TO OPERATIONS & $\stackrel{\circ}{\circ}$ & $\sum_{\substack{1 \\
\frac{1}{2}}}^{\frac{1}{2}}$ & $\begin{array}{l}\text { O } \\
\text { N } \\
\text { O }\end{array}$ & 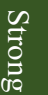 & $\stackrel{\circ}{\stackrel{0}{0}}$ & 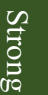 & 0 & 0 & - & $\underset{\omega}{i}$ & iे & i̊ \\
\hline$\checkmark$ & $\begin{array}{l}\text { Anticipating and Controlling Latent } \\
\text { Singles }\end{array}$ & + & PREOCCUPATION WITH FAILURE & $\stackrel{0}{\underset{\omega}{\sim}}$ & $\underset{\substack{0 \\
\tilde{E}}}{\infty}$ & : & $\begin{array}{l}\frac{1}{0} \\
\frac{2}{0} \\
\frac{2}{3} \\
\frac{2}{2}\end{array}$ & $\begin{array}{l}\stackrel{0}{+} \\
\dot{a} \\
\dot{A}\end{array}$ & $\begin{array}{l}\frac{5}{8} \\
\frac{9}{0} \\
\frac{9}{3} \\
\frac{3}{2}\end{array}$ & 0 & 0 & - & i & ஜ் & ஜे \\
\hline$\infty$ & Complacency & + & Misestimating Reliability & $\stackrel{\circ}{\stackrel{\sim}{ \pm}}$ & $\underset{\mathscr{C}}{\mathscr{0}}$ & : & $\begin{array}{l}\frac{3}{0} \\
\frac{0}{9} \\
\frac{0}{3}\end{array}$ & : & $\frac{3}{8}$ & 0 & 0 & - & i̊ & ஜे & ஜे \\
\hline$\bullet$ & Complacency & + & Normalization & $\stackrel{\circ}{\dot{0}}$ & 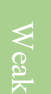 & $\underbrace{\stackrel{\circ}{\omega}}_{\omega}$ & $\sum_{\substack{\pi \\
\frac{\pi}{2}}}$ & 只 & $\begin{array}{l}3 \\
\frac{0}{2} \\
\frac{1}{3} \\
0\end{array}$ & - & 0 & 0 & i & i & ㅇ. \\
\hline$\varpi$ & Complacency & - & $\begin{array}{l}\text { Adherence to Standards and Risk } \\
\text { Boundaries }\end{array}$ & $\begin{array}{l}\circ \\
\dot{\circ} \\
\dot{+}\end{array}$ & 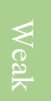 & $\stackrel{\circ}{\stackrel{0}{ \pm}}$ & $\begin{array}{l}3 \\
0 \\
0 \\
\frac{0}{9} \\
3 \\
3\end{array}$ & $\dot{\varphi}_{\substack{1 \\
\infty}}$ & $\begin{array}{l}3 \\
\frac{3}{8} \\
\frac{0}{9} \\
\text { di }\end{array}$ & 0 & 0 & - & $\underset{\omega}{\dot{\omega}}$ & ஜ் & ஷे \\
\hline ニ & Complacency & - & Listening to Various Voices and Inputs & $\stackrel{0}{\dot{1}}$ & $\underset{\substack{l \\
\frac{1}{\pi}}}{\sum}$ & $\underbrace{}_{\substack{i \\
\text { i }}}$ & 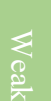 & $\stackrel{0}{\stackrel{0}{+}}$ & $\begin{array}{l}\frac{3}{0} \\
\frac{2}{0} \\
\frac{0}{0} \\
03\end{array}$ & - & 0 & 0 & $\dot{\omega}$ & i & டे \\
\hline
\end{tabular}

ư 


\begin{tabular}{|c|c|c|c|c|c|c|c|c|c|c|c|c|c|c|c|}
\hline $\bar{N}$ & Complacency & - & Precaution as a Mind-Set & 客 & 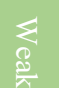 & $i_{\substack{\infty \\
\hdashline}}^{\infty}$ & $\underset{\substack{0 \\
\frac{1}{\pi}}}{\frac{1}{2}}$ & 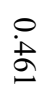 & $\begin{array}{ll}\frac{1}{0} \\
\frac{0}{0} \\
\frac{0}{30}\end{array}$ & - & 0 & 0 & is & i & 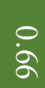 \\
\hline$\omega$ & Complacency & - & $\begin{array}{l}\text { Anticipating and Controlling Latent } \\
\text { Singles }\end{array}$ & $\stackrel{\circ}{\dot{u}}$ & 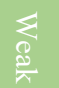 & $\stackrel{\circ}{\stackrel{\perp}{N}}$ & $\sum_{\substack{1 \\
\frac{1}{\pi}}}^{\frac{1}{2}}$ & : & 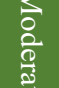 & - & 0 & 0 & i & i & ஜ் \\
\hline $\bar{\perp}$ & Compressed Experience & + & Domain Specific Experience & 这 & $\begin{array}{l}\text { 足 } \\
\bar{E}\end{array}$ & $\begin{array}{l}\dot{\text { in }} \\
\dot{0}\end{array}$ & $\begin{array}{l}\frac{1}{0} \\
\frac{0}{0}\end{array}$ & $\stackrel{\dot{\vec{U}}}{\vec{\Delta}}$ & $\begin{array}{l}\mathscr{n} \\
\overrightarrow{\tilde{E}}\end{array}$ & 0 & - & 0 & i: & ஷे & i \\
\hline $\bar{u}$ & Deviance of Attention & - & Anchoring in the Present & $\stackrel{\circ}{\Xi}$ & $\begin{array}{l}\frac{1}{0} \\
\frac{0}{3}\end{array}$ & $\underset{\omega}{\stackrel{\omega}{\omega}}$ & 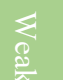 & : & $\sum_{\substack{x \\
\frac{\delta}{x}}}^{2}$ & 0 & 0 & - & ஷे & is & i \\
\hline$\sigma$ & Deviance of Attention & - & $\begin{array}{l}\text { Anticipating and Controlling Latent } \\
\text { Singles }\end{array}$ & $\stackrel{\circ}{\stackrel{\circ}{D}}$ & 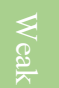 & $\dot{i}_{\breve{\omega}}$ & $\sum_{\frac{1}{2}}^{\frac{1}{2}}$ & $\begin{array}{l}0 \\
\text { 定 } \\
\text { u }\end{array}$ & 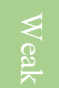 & - & - & - & i & is & i \\
\hline$\Xi$ & Distinctions & - & $\begin{array}{l}\text { Obscuring and Neglecting Significant } \\
\text { Information }\end{array}$ & $\begin{array}{l}0 \\
\dot{0}\end{array}$ & 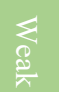 & $\begin{array}{l}0 \\
\dot{\omega} \\
\pm\end{array}$ & $\begin{array}{l}\sum \\
\frac{0}{2}\end{array}$ & 足 & 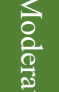 & - & 0 & 0 & $\dot{\omega}$ & is & ă \\
\hline$\infty$ & Domain Specific Experience & + & DEFERENCE TO EXPERTISE & 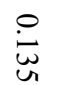 & $\begin{array}{l}\frac{1}{0} \\
\frac{0}{0} \\
\frac{0}{3}\end{array}$ & $\begin{array}{l}\stackrel{0}{\text { N }} \\
\text { 吾 }\end{array}$ & $\begin{array}{l}\sum \\
\frac{\mathbb{d}}{\alpha}\end{array}$ & $\dot{\omega}_{0}^{\omega}$ & $\frac{d}{\frac{1}{2}}$ & 0 & 0 & - & के & is & \\
\hline
\end{tabular}

๖̊ 


\begin{tabular}{|c|c|c|c|c|c|c|c|c|c|c|c|c|c|c|c|}
\hline$\varpi$ & Domain Specific Experience & + & Relative Expertise & 용 & $\begin{array}{l}\sum \\
\frac{\sum}{\pi} \\
\frac{8}{2}\end{array}$ & $\stackrel{\circ}{\stackrel{ \pm}{W}}$ & $\frac{3}{8}$ & $\stackrel{\circ}{\infty}$ & $\begin{array}{ll}\frac{1}{0} \\
\frac{0}{0} \\
\frac{0}{30}\end{array}$ & 0 & $\circ$ & - & 出 & ஜे & ஷे \\
\hline ת & Domain Specific Experience & + & Personal Competence and Confidence & $\begin{array}{l}\dot{2} \\
\dot{\infty}\end{array}$ & $\begin{array}{l}\sum \\
\frac{8}{n}\end{array}$ & $\begin{array}{l}0 \\
\dot{\omega} \\
\dot{\Phi}\end{array}$ & $\begin{array}{l}\sum \\
\frac{0}{N}\end{array}$ & 总 & $\begin{array}{l}\overrightarrow{0} \\
\overrightarrow{0} \\
0 \\
0\end{array}$ & - & 0 & 0 & i & i & ஜे \\
\hline$\simeq$ & Domain Specific Experience & + & Improvisation & $\dot{0}$ & 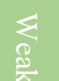 & $\begin{array}{l}\dot{i} \\
\dot{\sigma}\end{array}$ & 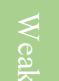 & 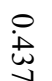 & $\begin{array}{l}0 \\
0 \\
0 \\
0 \\
0\end{array}$ & - & 0 & o & ¿ & ¿ & के \\
\hline N & Domain Specific Experience & + & Responses (Actions) Repertoire & $\stackrel{\dot{0}}{\dot{0}}$ & $\begin{array}{l}\sum \\
\frac{\mathbb{R}}{x}\end{array}$ & $\frac{\dot{\omega}}{a}$ & 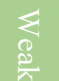 & $\begin{array}{l}\stackrel{P}{ \pm} \\
\stackrel{+}{u}\end{array}$ & $\begin{array}{l}\sum \\
\frac{d}{\pi}\end{array}$ & - & - & - & i & i & is \\
\hline 心 & Domain Specific Experience & + & Sensemaking & $\stackrel{\dot{0}}{\dot{1}}$ & 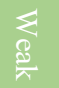 & 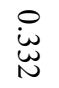 & $\begin{array}{l}\sum \\
\frac{1}{2} \\
\frac{D}{\pi}\end{array}$ & 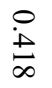 & 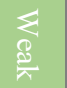 & - & - & - & i & is & is \\
\hline$\tilde{A}$ & Domain Specific Experience & + & $\begin{array}{l}\text { Anticipating and Controlling Latent } \\
\text { Singles }\end{array}$ & $\stackrel{\circ}{\dot{0}}$ & 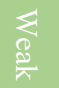 & $\stackrel{\circ}{+}$ & $\begin{array}{l}\sum \\
\frac{1}{2} \\
\frac{8}{\pi}\end{array}$ & $\stackrel{\stackrel{P}{+}}{\stackrel{\infty}{\infty}}$ & $\frac{0}{0}$ & - & 0 & 0 & 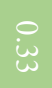 & is & ஷे \\
\hline 屾 & Domain Specific Experience & + & Logical Reasoning & 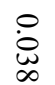 & 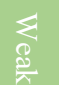 & $\underset{\dot{\omega}}{\dot{\omega}}$ & $\frac{2}{8}$ & $\stackrel{\stackrel{P}{ \pm}}{ }$ & $\begin{array}{l}\frac{1}{0} \\
\frac{2}{0} \\
0\end{array}$ & - & 0 & o & $i_{\omega}$ & i & aे \\
\hline
\end{tabular}

$\stackrel{\omega}{9}$ 


\begin{tabular}{|c|c|c|c|c|c|c|c|c|c|c|c|c|c|c|c|}
\hline న & Domain Specific Experience & + & $\begin{array}{l}\text { Sustaining Dynamic Situational } \\
\text { Awareness }\end{array}$ & $\stackrel{\circ}{\dot{D}}$ & $\underset{\substack{\alpha \\
\frac{8}{2}}}{\frac{1}{2}}$ & $\stackrel{\infty}{\infty}_{\infty}^{\infty}$ & $\underset{\substack{\infty \\
\infty}}{\frac{1}{2}}$ & $\underset{0}{i}$ & $\underset{\substack{0 \\
\frac{0}{K}}}{\frac{1}{2}}$ & - & - & - & i & i & i \\
\hline ב & Domain Specific Experience & - & Pressure & $\stackrel{\circ}{\stackrel{ }{*}}$ & $\underset{\substack{k \\
0}}{\frac{1}{\pi}}$ & 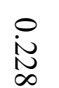 & $\begin{array}{l}\sum \\
\infty \\
\frac{\pi}{1}\end{array}$ & $\begin{array}{l}\stackrel{0}{N} \\
\stackrel{+}{a}\end{array}$ & $\sum_{0}^{\frac{1}{\pi}}$ & - & - & - & is & i & i \\
\hline$\underset{\infty}{\infty}$ & Domain Specific Experience & + & Learning from Failure & $\stackrel{\circ}{\stackrel{\circ}{N}}$ & $\underset{\substack{k \\
0}}{\frac{1}{\pi}}$ & 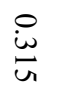 & 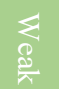 & $\begin{array}{l}\dot{0} \\
\dot{0} \\
\dot{\infty}\end{array}$ & $\sum_{\substack{\alpha \\
\frac{0}{N}}}^{2}$ & - & - & - & i & i & is \\
\hline N & Dysfunctional Momentum & - & SENSITIVITY TO OPERATIONS & $\stackrel{\circ}{\dot{\omega}}$ & $\underset{\substack{2 \\
\frac{1}{2}}}{\frac{2}{2}}$ & $i_{\dot{w}}^{\infty}$ & 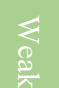 & $\stackrel{\circ}{ \pm}$ & $\sum_{\substack{\frac{1}{R} \\
\frac{3}{\pi}}}$ & - & - & - & is & i & is \\
\hline 。 & Elasticity and Recovery & + & System Flexibility and Adaptability & $\stackrel{\circ}{\infty}$ & $\begin{array}{ll}\frac{0}{0} \\
\frac{0}{0} \\
\frac{9}{3} \\
3\end{array}$ & $\stackrel{\circ}{\stackrel{\leftrightarrow}{\omega}}$ & $\begin{array}{l}\frac{8}{0} \\
\frac{0}{0} \\
\frac{9}{3}\end{array}$ & $\begin{array}{l}\dot{0} \\
\dot{\leftrightarrow} \\
\dot{b}\end{array}$ & 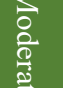 & - & - & - & à & ஷे & aे \\
\hline$\omega$ & Failure Knowledge and Attentiveness & + & $\begin{array}{l}\text { Anticipating and Controlling Latent } \\
\text { Singles }\end{array}$ & $\stackrel{\circ}{\dot{E}}$ & 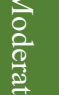 & $\begin{array}{l}\text { in } \\
\text { ì }\end{array}$ & 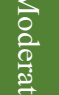 & $\stackrel{\odot}{\dot{\sim}}$ & 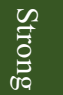 & - & 0 & 0 & के & के & $i$ \\
\hline$\stackrel{\sim}{N}$ & Failure Knowledge and Attentiveness & + & Logical Reasoning & $\stackrel{\circ}{\dot{3}}$ & $\underset{\substack{\infty \\
0}}{\frac{0}{2}}$ & 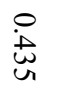 & $\begin{array}{l}\frac{8}{0} \\
\frac{0}{9} \\
\frac{9}{3}\end{array}$ & ì & $\begin{array}{l}0 \\
0 \\
0 \\
0 \\
03 \\
3\end{array}$ & 0 & 0 & - & $i$ & ட் & aे \\
\hline
\end{tabular}

$\underset{\infty}{\infty}$ 


\begin{tabular}{|c|c|c|c|c|c|c|c|c|c|c|c|c|c|c|c|}
\hline$\omega$ & Headful Interrelating & + & SENSITIVITY TO OPERATIONS & $\stackrel{\circ}{\dot{1}}$ & $\sum_{\substack{\infty \\
e}}$ & i & 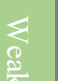 & i & $\sum_{\substack{0 \\
0}}$ & - & - & - & is & is & is \\
\hline$\stackrel{\omega}{\perp}$ & Heedful Interrelating & + & Interruptions and Adjustments & : & 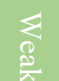 & 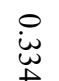 & 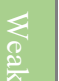 & $\underset{\text { i }}{\stackrel{D}{u}}$ & $\underset{\frac{1}{2}}{\frac{1}{2}}$ & - & - & - & $i_{\omega}$ & $i_{\omega}$ & i \\
\hline$w_{i}$ & Heedful Interrelating & + & $\begin{array}{l}\text { Anticipating and Controlling Latent } \\
\text { Singles }\end{array}$ & $\stackrel{\circ}{\stackrel{0}{0}}$ & $\sum_{\substack{x \\
\frac{1}{\pi}}}^{\sum}$ & $\underset{\dot{\infty}}{\dot{\phi}}$ & $\sum_{\substack{\pi \\
\frac{1}{2}}}^{2}$ & $\stackrel{\stackrel{\oplus}{ \pm}}{\omega}$ & 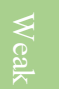 & - & - & - & i & is & $\begin{array}{c}0 \\
i \omega \\
i\end{array}$ \\
\hline & Improvisation & + & COMMITMENT TO RESILIENCE & $\stackrel{0}{9}$ & $\frac{0}{0}$ & $\dot{i}_{0}$ & $\sum_{\substack{0 \\
0}}^{2}$ & 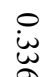 & $\sum_{0}^{\infty}$ & 0 & 0 & - & த் & is & $\overbrace{\omega}^{\infty}$ \\
\hline$\underline{y}$ & Improvisation & + & $\begin{array}{l}\text { Action Interpretation and Recursive } \\
\text { Interaction }\end{array}$ & $\dot{\dot{\theta}}$ & $\underset{\substack{k \\
\frac{0}{2}}}{\frac{2}{2}}$ & 总 & $\sum_{\substack{\pi \\
\frac{1}{2}}}$ & $\underset{\substack{i \\
\infty}}{\infty}$ & $\sum_{\substack{1 \\
\frac{1}{2}}}^{2}$ & - & - & - & i & is & is \\
\hline$\omega$ & Interruptions and Adjustments & - & Dysfunctional Momentum & $\begin{array}{l}\stackrel{0}{\tilde{N}} \\
\dot{I}\end{array}$ & 里 & $\underset{\substack{\infty \\
\dot{\infty}}}{\stackrel{\infty}{\infty}}$ & $\begin{array}{ll}\frac{1}{0} \\
\frac{0}{9} \\
\frac{9}{3}\end{array}$ & $\dot{a}$ & $\begin{array}{ll}\frac{1}{2} \\
\frac{0}{0} \\
\frac{0}{3}\end{array}$ & 0 & 0 & - & : & ஜे & ஷे \\
\hline$\omega$ & Interruptions and Adjustments & + & $\begin{array}{l}\text { Anticipating and Controlling Latent } \\
\text { Singles }\end{array}$ & $\begin{array}{l}0 \\
\dot{0} \\
\text { i }\end{array}$ & $\underset{\substack{2 \\
\frac{1}{\pi}}}{\frac{2}{\pi}}$ & 虽 & $\underset{\mathbb{2}}{\frac{1}{\pi}}$ & $\underset{\substack{\infty \\
\infty \\
\infty}}{\stackrel{0}{\infty}}$ & 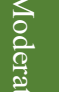 & - & 0 & 0 & i & i & ஷे \\
\hline
\end{tabular}




\begin{tabular}{|c|c|c|c|c|c|c|c|c|c|c|c|c|c|c|c|}
\hline$b$ & Learning from Failure & + & COMMITMENT TO RESILIENCE & $\stackrel{\circ}{\stackrel{P}{\perp}}$ & $\begin{array}{l}\frac{3}{8} \\
\frac{0}{0} \\
\frac{0}{3}\end{array}$ & 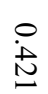 & $\begin{array}{l}\sum \\
\frac{1}{2} \\
\frac{8}{2}\end{array}$ & $\begin{array}{l}\text { o } \\
\dot{\leftrightarrow}\end{array}$ & 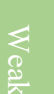 & 0 & 0 & - & 음 & i & $\underset{\omega}{i}$ \\
\hline \pm & Learning from Failure & + & Responses (Actions) Repertoire & $\stackrel{\circ}{\circ}$ & $\frac{1}{8}$ & $\stackrel{\circ}{\stackrel{i}{N}}$ & $\begin{array}{l}\sum \\
\frac{8}{2} \\
\frac{8}{2}\end{array}$ & 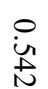 & $\begin{array}{l}\frac{1}{8} \\
\frac{0}{9} \\
\frac{0}{0}\end{array}$ & 0 & - & 0 & ஷे & $i_{\omega}$ & $\dot{\alpha}$ \\
\hline$\hat{N}$ & Learning from Failure & + & Improvisation & $\stackrel{\circ}{8}$ & $\begin{array}{l}\frac{1}{8} \\
\frac{3}{\pi}\end{array}$ & $\underset{\substack{\infty \\
\infty \\
\infty}}{0}$ & $\frac{\sum}{8}$ & $\begin{array}{l}\circ \\
\text { 壬 }\end{array}$ & $\begin{array}{l}\frac{1}{8} \\
\frac{0}{0} \\
\frac{0}{30} \\
0.7\end{array}$ & - & 0 & 0 & is & i & 요 \\
\hline$\underline{\omega}$ & Learning from Failure & + & Failure Knowledge and Attentiveness & $\dot{\circ}$ & $\begin{array}{l}2 \\
\frac{3}{2} \\
\frac{3}{\pi}\end{array}$ & $\stackrel{\circ}{\stackrel{0}{0}}$ & $\frac{3}{8}$ & $\stackrel{\circ}{\dot{a}}$ & $\frac{3}{2}$ & 0 & 0 & - & $\underset{\omega}{\text { i }}$ & ஜे & 여 \\
\hline$\stackrel{A}{A}$ & Listening to Various Voices and Inputs & + & Precaution as a Mind-Set & $\stackrel{\circ}{\circ}$ & $\begin{array}{l}\sum \\
\frac{\pi}{2} \\
\frac{0}{\pi}\end{array}$ & $\stackrel{\circ}{\stackrel{\oplus}{ \pm}}$ & $\begin{array}{l}\frac{3}{3} \\
\frac{3}{0} \\
\frac{3}{3} \\
3\end{array}$ & $\stackrel{\circ}{\dot{a}}$ & 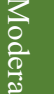 & 0 & 0 & - & i & ஜे & 영 \\
\hline 究 & Listening to Various Voices and Inputs & + & Failure Knowledge and Attentiveness & $\begin{array}{l}\circ \\
\dot{N} \\
\dot{N}\end{array}$ & $\begin{array}{l}\sum \\
\frac{1}{2} \\
\frac{D}{\pi}\end{array}$ & $\begin{array}{l}\text { in } \\
\text { in }\end{array}$ & 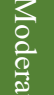 & $\dot{0}$ & $\begin{array}{l}\frac{3}{0} \\
\frac{0}{2} \\
\frac{9}{9} \\
03\end{array}$ & 0 & 0 & - & i & ஜे & 여 \\
\hline 古 & Listening to Various Voices and Inputs & + & $\begin{array}{l}\text { Anticipating and Controlling Latent } \\
\text { Singles }\end{array}$ & $\stackrel{\circ}{\stackrel{2}{2}}$ & 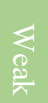 & $\stackrel{\circ}{i}$ & 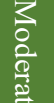 & $\begin{array}{l}\stackrel{0}{\dot{+}} \\
\dot{+1}\end{array}$ & $\begin{array}{l}\frac{3}{8} \\
\frac{1}{9} \\
03 \\
03\end{array}$ & 0 & 0 & - & is & ஜे & $\dot{a}$ \\
\hline
\end{tabular}

$\omega$ 


\begin{tabular}{|c|c|c|c|c|c|c|c|c|c|c|c|c|c|c|c|}
\hline \pm & Listening to Various Voices and Inputs & - & $\begin{array}{l}\text { Obscuring and Neglecting Significant } \\
\text { Information }\end{array}$ & $\stackrel{\circ}{\stackrel{0}{\infty}}$ & $\underset{\substack{2 \\
\frac{8}{2}}}{\frac{d}{2}}$ & i & $\begin{array}{l}\sum \\
0 \\
\frac{0}{\pi}\end{array}$ & 运 & 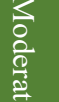 & - & 0 & 0 & is & i & ஷे \\
\hline$\infty$ & Listening to Various Voices and Inputs & - & Normalization & : & $\sum_{\substack{1 \\
\frac{1}{x}}}$ & $\dot{\omega}_{i}$ & 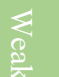 & $\stackrel{P}{\stackrel{D}{ \pm}}$ & 产 & - & o & 0 & i & $\omega_{\omega}$ & a \\
\hline to & Logical Reasoning & + & $\begin{array}{l}\text { Sustaining Dynamic Situational } \\
\text { Awareness }\end{array}$ & $\stackrel{\circ}{8}$ & 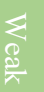 & $\stackrel{\circ}{\stackrel{\infty}{\omega}}$ & $\frac{\pi}{8}$ & $\underset{\substack{i \\
\dot{\omega}}}{\stackrel{0}{L}}$ & $\sum_{\substack{\alpha \\
\frac{0}{2}}}^{2}$ & - & - & - & is & is & i \\
\hline 岁 & Logical Reasoning & + & Operations as an Integrated Map & $\dot{0}$ & 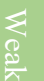 & î̃ & $\underset{0}{\frac{1}{R}}$ & 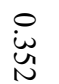 & $\frac{2}{9}$ & - & - & - & i & is & \\
\hline 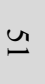 & Logical Reasoning & + & Sensemaking & $\dot{0}$ & 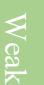 & iu & $\underset{\substack{1 \\
\frac{\delta}{2}}}{\frac{1}{2}}$ & 总 & $\underset{\frac{1}{\alpha}}{\frac{1}{x}}$ & - & - & - & i & i & 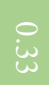 \\
\hline 岕 & Logical Reasoning & + & Distinctions & $\stackrel{0}{\dot{\omega}}$ & 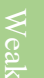 & $\stackrel{\text { i d }}{\text { i }}$ & $\underset{\substack{k \\
\infty}}{\frac{1}{x}}$ & $\begin{array}{l}\dot{\omega}_{u} \\
\dot{\omega}\end{array}$ & 2 & - & - & - & $\omega$ & is & \\
\hline 岕 & Logical Reasoning & + & $\begin{array}{l}\text { Anticipating and Controlling Latent } \\
\text { Singles }\end{array}$ & $\stackrel{\circ}{\stackrel{0}{0}}$ & 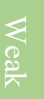 & $\stackrel{\odot}{ \pm}$ & $\begin{array}{ll}0 \\
0 \\
0 \\
0 \\
\frac{2}{3}\end{array}$ & : & 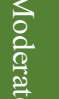 & 0 & 0 & - & $w$ & à & \\
\hline
\end{tabular}

 


\begin{tabular}{|c|c|c|c|c|c|c|c|c|c|c|c|c|c|c|c|}
\hline 崩 & Logical Reasoning & - & $\begin{array}{l}\text { Obscuring and Neglecting Significant } \\
\text { Information }\end{array}$ & $\stackrel{\circ}{\circ}$ & 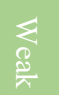 & iu & 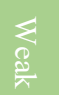 & $\stackrel{\circ}{ \pm}$ & 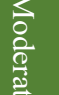 & - & 0 & 0 & is & i & ஜे \\
\hline ü & Loss of Situational Awareness & - & Operations as an Integrated Map & $\stackrel{0}{0}$ & $\underset{\substack{i \\
\infty}}{\frac{1}{x}}$ & i & 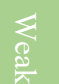 & $\underset{\substack{\infty \\
\infty}}{\stackrel{\infty}{\infty}}$ & $\underset{\frac{1}{\alpha}}{\frac{1}{\alpha}}$ & - & - & - & i & $i_{\omega}^{i}$ & i \\
\hline ू̆ & Loss of Situational Awareness & + & $\begin{array}{l}\text { Obscuring and Neglecting Significant } \\
\text { Information }\end{array}$ & 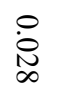 & $\underset{\substack{k \\
\frac{\delta}{x}}}{\sum}$ & i & $\underset{\substack{i \\
\frac{1}{x}}}{\frac{1}{x}}$ & $\stackrel{\odot}{\infty}$ & $\underset{\substack{i \\
\frac{\delta}{\alpha}}}{\frac{1}{2}}$ & - & - & - & i & i & i \\
\hline un & Members' Level of Participation & + & Heedful Interrelating & 芯 & 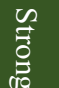 & $\underset{\infty}{+}$ & 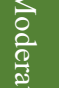 & $\stackrel{\circ}{\grave{d}}$ & 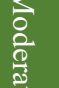 & 0 & 0 & - & i: & के & aे \\
\hline 岕 & Minimizing Expertise & - & Relative Expertise & $\stackrel{\stackrel{O}{N}}{=}$ & $\begin{array}{ll}0 \\
0 \\
\frac{9}{9} \\
\frac{9}{3}\end{array}$ & $\underset{\dot{n}}{\dot{\leftrightarrow}}$ & $\begin{array}{ll}0 \\
0 \\
\frac{9}{3} \\
\frac{3}{3}\end{array}$ & $\begin{array}{l}0 \\
\dot{\theta} \\
\dot{g}\end{array}$ & $\begin{array}{ll}\frac{0}{0} \\
\frac{9}{3} \\
\frac{9}{3} \\
3\end{array}$ & - & - & - & के & aे & aे \\
\hline 냉 & Misestimating Reliability & - & $\begin{array}{l}\text { Anticipating and Controlling Latent } \\
\text { Singles }\end{array}$ & $\stackrel{\circ}{\stackrel{\circ}{u}}$ & 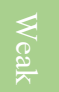 & $\underset{\sim}{\stackrel{i}{N}}$ & $\underset{\substack{\infty \\
\frac{\mathbb{R}}{2}}}{\sum}$ & $\underset{\stackrel{+}{+}}{\stackrel{+}{\infty}}$ & 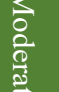 & - & 0 & 0 & i & i & ஜे \\
\hline 8 & Misestimating Reliability & - & PREOCCUPATION WITH FAILURE & : & 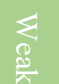 & 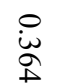 & 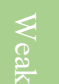 & 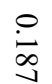 & $\frac{2}{8}$ & - & - & - & i & i & \\
\hline
\end{tabular}

N 


\begin{tabular}{|c|c|c|c|c|c|c|c|c|c|c|c|c|c|c|c|}
\hline 2 & Normalization & + & $\begin{array}{l}\text { Obscuring and Neglecting Significant } \\
\text { Information }\end{array}$ & $\stackrel{\circ}{\dot{\leftrightarrow}}$ & 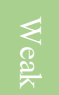 & $\underset{\substack{\infty \\
\infty}}{\stackrel{\infty}{\infty}}$ & 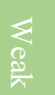 & $\underset{\substack{\infty \\
\infty}}{\substack{\infty \\
\infty}}$ & $\underset{\infty}{\sum}$ & - & - & - & is & is & i \\
\hline నิ & Normalization & - & $\begin{array}{l}\text { Anticipating and Controlling Latent } \\
\text { Singles }\end{array}$ & $\stackrel{\circ}{\stackrel{0}{0}}$ & $\begin{array}{l}\sum \\
\frac{8}{\pi} \\
\frac{8}{\pi}\end{array}$ & 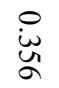 & $\underset{\substack{2 \\
\frac{1}{\pi}}}{\frac{1}{2}}$ & $\underset{\substack{+\infty \\
+\infty}}{\stackrel{\infty}{+}}$ & 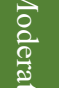 & - & 0 & 0 & is & $\begin{array}{c}0 \\
i \infty \\
i \infty\end{array}$ & के \\
\hline ¿ & Normalization & - & PREOCCUPATION WITH FAILURE & $\stackrel{\dot{0}}{\mathrm{i}}$ & 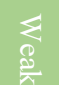 & $\underset{\sim}{\stackrel{\sim}{N}}$ & $\underset{\frac{\delta}{\alpha}}{\frac{\sum}{\alpha}}$ & $\begin{array}{l}\dot{\tilde{O}} \\
\text { ó }\end{array}$ & $\underset{\substack{k \\
\frac{\delta}{x}}}{\sum}$ & - & - & - & is & is & $\stackrel{\omega}{\omega}$ \\
\hline I & $\begin{array}{l}\text { Obscuring and Neglecting Significant } \\
\text { Information }\end{array}$ & - & RELUCTANCE TO SIMPLIFY & $\stackrel{\circ}{\stackrel{\sim}{\sim}}$ & $\underset{0}{\mathscr{E}}$ & $i_{0}^{0}$ & $\underset{\substack{\infty \\
\frac{\mathbb{R}}{2}}}{\infty}$ & $\stackrel{0}{\underset{U}{\omega}}$ & 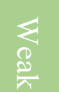 & 0 & 0 & - & 8 & i & $i$ \\
\hline ऊे & $\begin{array}{l}\text { Obscuring and Neglecting Significant } \\
\text { Information }\end{array}$ & - & $\begin{array}{l}\text { Anticipating and Controlling Latent } \\
\text { Singles }\end{array}$ & $\stackrel{\circ}{\dot{m}}$ & $\underset{\substack{k \\
\frac{d}{\pi}}}{\frac{d}{\pi}}$ & 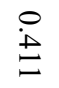 & 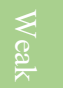 & 总 & $\begin{array}{l}\overrightarrow{0} \\
\stackrel{0}{0} \\
\stackrel{0}{3}\end{array}$ & - & 0 & 0 & is & is & aे \\
\hline ๙ & Operations as an Integrated Map & + & SENSITIVITY TO OPERATIONS & $\stackrel{\circ}{\dot{\omega}}$ & 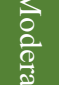 & $\begin{array}{l}\stackrel{P}{2} \\
\text { 芯 }\end{array}$ & $\sum$ & $\underset{\text { i }}{\stackrel{i}{w}}$ & 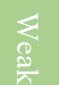 & 0 & 0 & - & $\dot{a}$ & i & is \\
\hline 9 & Organizing Action Based Inquiry & + & RELUCTANCE TO SIMPLIFY & 客 & $\sum_{\substack{i \\
\frac{1}{1}}}$ & $\underset{\substack{\dot{u} \\
\hdashline}}{u}$ & $\underset{\substack{i \\
0}}{\frac{\delta_{n}}{2}}$ & i & 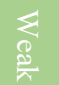 & - & - & - & i & ¿ & i \\
\hline
\end{tabular}

w 


\begin{tabular}{|c|c|c|c|c|c|c|c|c|c|c|c|c|c|c|c|}
\hline$\infty$ & Organizing Action Based Inquiry & - & $\begin{array}{l}\text { Obscuring and Neglecting Significant } \\
\text { Information }\end{array}$ & 号 & 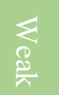 & 它 & $\sum_{\substack{\infty \\
\infty}}^{\infty}$ & $\underset{\dot{\infty}}{\infty}$ & $\frac{\sum}{8}$ & - & - & - & i & i & i \\
\hline 8 & Organizing for Sense-Discrediting & + & Precaution as a Mind-Set & $\stackrel{i}{i}$ & 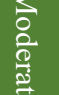 & 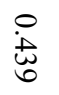 & $\begin{array}{l}\frac{1}{0} \\
\frac{0}{0} \\
\frac{0}{20}\end{array}$ & ĭ & 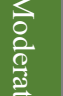 & - & - & - & ஷे & ஷे & ஜे \\
\hline ฮે & Organizing for Sense-Discrediting & - & $\begin{array}{l}\text { Obscuring and Neglecting Significant } \\
\text { Information }\end{array}$ & $\stackrel{\circ}{\dot{D}}$ & $\underset{\substack{1 \\
\mathbb{N}}}{\frac{1}{\pi}}$ & $\stackrel{\circ}{i}$ & 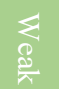 & 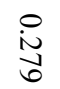 & $\sum_{\substack{\alpha \\
\frac{0}{N}}}^{2}$ & - & - & - & i & i & is \\
\hline$\checkmark$ & Organizing for Sense-Discrediting & - & Normalization & 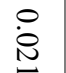 & $\sum_{\substack{1 \\
\infty}}^{\infty}$ & $\stackrel{0}{0}$ & $\sum_{\substack{1 \\
\infty}}$ & $\stackrel{0}{i}$ & 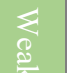 & - & - & - & is & is & i \\
\hline N & Organizing for Sense-Discrediting & + & Sensemaking & $\stackrel{\circ}{0}$ & 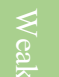 & $\underset{\sim}{\stackrel{0}{\omega}}$ & $\sum_{\substack{x \\
\frac{1}{x}}}$ & 这 & $\sum_{0}^{\infty}$ & - & - & - & is & is & ๘ \\
\hline$\vec{\omega}$ & Organizing for Sense-Discrediting & + & RELUCTANCE TO SIMPLIFY & $\stackrel{\circ}{\stackrel{0}{N}}$ & $\underset{\substack{\infty \\
\infty}}{\frac{1}{x}}$ & $\stackrel{i}{\dot{\omega}}$ & $\underset{\substack{k \\
0 \\
\frac{\delta}{x}}}{2}$ & $\stackrel{\circ}{0}$ & 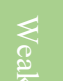 & - & - & - & is & is & is \\
\hline$\vec{\perp}$ & Organizing for Variety & + & RELUCTANCE TO SIMPLIFY & : & 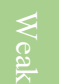 & $\underset{\substack{\infty \\
\sim}}{i}$ & 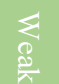 & $\underset{\substack{\infty \\
N}}{\stackrel{0}{\infty}}$ & $\underset{\frac{1}{2}}{\frac{1}{2}}$ & - & - & - & is & i & 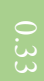 \\
\hline
\end{tabular}

$\stackrel{\omega}{D}$ 


\begin{tabular}{|c|c|c|c|c|c|c|c|c|c|c|c|c|c|c|c|}
\hline ù & Personal Competence and Confidence & + & Improvisation & $\stackrel{\circ}{\stackrel{0}{0}}$ & $\sum_{\substack{\pi \\
\frac{\delta}{\pi}}}$ & $\underset{\substack{\infty \\
\text { 市 }}}{ }$ & $\begin{array}{l}\sum_{\mathbb{2}} \\
\frac{\mathbb{R}}{\pi}\end{array}$ & $\underbrace{\underline{\omega}}_{0}$ & 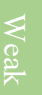 & - & - & - & $i_{\omega}$ & i. & $\underset{\omega}{i}$ \\
\hline নু & Precaution as a Mind-Set & + & $\begin{array}{l}\text { Anticipating and Controlling Latent } \\
\text { Singles }\end{array}$ & $\stackrel{0}{\stackrel{0}{+}}$ & $\sum_{\substack{\infty \\
\frac{8}{2}}}^{\frac{1}{2}}$ & 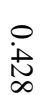 & $\underset{\substack{1 \\
8}}{\frac{1}{\pi}}$ & 沜 & $\begin{array}{l}\frac{3}{8} \\
\frac{2}{2} \\
\frac{0}{0}\end{array}$ & - & 0 & 0 & i & $\underset{\omega}{\text { i }}$ & ஷे \\
\hline ป & Precaution as a Mind-Set & + & PREOCCUPATION WITH FAILURE & $\stackrel{\circ}{\circ}$ & $\underset{\substack{1 \\
\frac{1}{2}}}{\frac{1}{2}}$ & 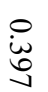 & $\underset{\substack{2 \\
\frac{1}{2}}}{\frac{1}{1}}$ & $\stackrel{\circ}{i}$ & $\underset{8}{\frac{1}{\pi}}$ & - & - & - & i & i & ij \\
\hline$\infty$ & Precaution as a Mind-Set & - & $\begin{array}{l}\text { Obscuring and Neglecting Significant } \\
\text { Information }\end{array}$ & $\stackrel{\circ}{\dot{\omega}}$ & $\underset{8}{\frac{1}{2}}$ & $\stackrel{\circ}{i}$ & $\underset{\mathbb{S}}{\frac{\mathbb{N}}{\pi}}$ & 足 & $\begin{array}{l}\frac{3}{0} \\
\frac{0}{0} \\
\frac{0}{20}\end{array}$ & - & 0 & 0 & i & i & ஷे \\
\hline చె & Precaution as a Mind-Set & - & Normalization & $\stackrel{-1}{\circ}$ & $\sum_{\substack{\infty \\
\frac{\pi}{2}}}^{\infty}$ & $\stackrel{\circ}{\underset{\omega}{\omega}}$ & $\sum_{\substack{1 \\
0}}^{\frac{1}{\pi}}$ & $\stackrel{\circ}{\stackrel{\leftrightarrow}{\omega}}$ & $\begin{array}{l}\frac{3}{0} \\
\frac{0}{0} \\
\frac{1}{30}\end{array}$ & - & 0 & 0 & i & i & ㅇ. \\
\hline$\infty$ & Precaution as a Mind-Set & + & Sensemaking & $\begin{array}{l}\circ \\
\dot{0} \\
\text { U }\end{array}$ & $\underset{\substack{1 \\
8 \\
\frac{0}{\pi}}}{\infty}$ & $\stackrel{\circ}{\stackrel{\circ}{\Xi}}$ & $\underset{\substack{1 \\
8}}{\frac{1}{x}}$ & $\underset{\substack{\infty \\
\infty}}{i}$ & $\underset{8}{\frac{1}{\pi}}$ & - & - & - & i & i. & $\underset{\omega}{\omega}$ \\
\hline$\infty$ & Precaution as a Mind-Set & - & Misestimating Reliability & $\stackrel{\circ}{\stackrel{0}{\infty}}$ & $\underset{\frac{8}{2}}{\frac{1}{\pi}}$ & $\begin{array}{l}0 \\
\dot{\omega} \\
\dot{b}\end{array}$ & $\begin{array}{l}\$ \\
8 \\
\frac{8}{2}\end{array}$ & $\begin{array}{l}\stackrel{0}{\sim} \\
\underset{\sim}{\infty}\end{array}$ & $\frac{8}{\pi}$ & - & - & - & i & iw & \\
\hline
\end{tabular}

$\underset{\omega}{\omega}$ 


\begin{tabular}{|c|c|c|c|c|c|c|c|c|c|c|c|c|c|c|c|}
\hline$\infty$ & Pressure & + & $\begin{array}{l}\text { Obscuring and Neglecting Significant } \\
\text { Information }\end{array}$ & $\begin{array}{l}\dot{0} \\
\dot{Q} \\
\text { 品 }\end{array}$ & $\sum_{\frac{0}{\pi}}^{\sum}$ & 芯 & $\underset{\substack{\infty \\
\frac{d}{2}}}{\sum}$ & i & $\underset{\substack{\alpha \\
\frac{d}{\alpha}}}{2}$ & - & - & - & i & $\begin{array}{c}\text { i } \\
\text { i }\end{array}$ & i \\
\hline$\stackrel{\infty}{\omega}$ & Pressure & - & SENSITIVITY TO OPERATIONS & $\begin{array}{l}\stackrel{0}{0} \\
\dot{1}\end{array}$ & 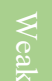 & $\begin{array}{l}\dot{i} \\
\dot{\sigma}\end{array}$ & $\begin{array}{l}\sum \\
\frac{\delta}{\alpha}\end{array}$ & 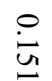 & $\begin{array}{l}\sum \\
\frac{8}{\alpha}\end{array}$ & - & - & - & $i_{\omega}$ & $i_{\omega}$ & i \\
\hline$\stackrel{\infty}{+}$ & Pressure & - & Distinctions & $\dot{~}$ & 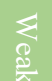 & 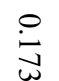 & 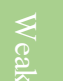 & $\stackrel{\circ}{\circ}$ & $\underset{\substack{1 \\
\frac{8}{x}}}{\frac{1}{x}}$ & - & - & - & i & is & i \\
\hline$\stackrel{\infty}{\hookrightarrow}$ & Pressure & - & Operations as an Integrated Map & : & $\sum_{\frac{d}{\alpha}}^{\infty}$ & 茫 & 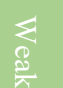 & $\stackrel{0}{\stackrel{N}{J}}$ & $\begin{array}{l}\sum \\
\frac{8}{2}\end{array}$ & - & - & - & ¿ & i & 谂 \\
\hline$\stackrel{\infty}{\infty}$ & Pressure & - & $\begin{array}{l}\text { Anticipating and Controlling Latent } \\
\text { Singles }\end{array}$ & 官 & $\underset{\substack{\infty \\
\frac{1}{\pi}}}{\frac{2}{\pi}}$ & $\underset{\infty}{\stackrel{\infty}{N}}$ & 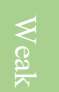 & $\stackrel{0}{\stackrel{U}{J}}$ & $\begin{array}{l}\sum \\
\frac{d}{\alpha} \\
\frac{d}{\pi}\end{array}$ & - & - & - & i & is & i \\
\hline$\stackrel{\infty}{v}$ & Reconstituting the Evolving Present & + & Sensemaking & $\dot{~}$ & $\underset{\substack{\alpha \\
\frac{d}{d}}}{2}$ & $\dot{i}_{\substack{u \\
t}}$ & 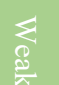 & $\underset{\text { iu }}{\stackrel{i}{u}}$ & $\sum$ & - & - & - & $i_{\omega}$ & i & i \\
\hline$\infty$ & Reconstituting the Evolving Present & + & Elasticity and Recovery & 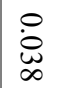 & $\sum_{\substack{0 \\
0}}^{\frac{1}{\pi}}$ & $\underset{\substack{\infty \\
+\infty}}{\stackrel{+}{\infty}}$ & 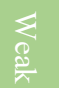 & 定 & 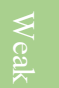 & - & - & - & i & is & is \\
\hline
\end{tabular}

a 


\begin{tabular}{|c|c|c|c|c|c|c|c|c|c|c|c|c|c|c|c|}
\hline$\infty$ & Redundancy & + & Complacency & $\stackrel{\circ}{8}$ & $\begin{array}{l}\sum \\
\frac{1}{\pi} \\
\frac{d}{n}\end{array}$ & 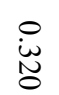 & $\underset{\substack{i \\
0}}{\frac{1}{\pi}}$ & 总 & $\begin{array}{l}3 \\
\frac{3}{2} \\
\frac{0}{9} \\
\frac{9}{9}\end{array}$ & - & 0 & 0 & is & i & 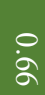 \\
\hline 8 & Redundancy & + & Organizing for Sense-Discrediting & $\dot{0}$ & 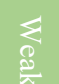 & 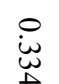 & $\underset{\infty}{\infty}$ & $\stackrel{i}{t}$ & $\begin{array}{l}\text { o } \\
\frac{0}{0} \\
\frac{0}{0}\end{array}$ & - & 0 & 0 & is & i & ஜे \\
\hline$\stackrel{0}{\circ}$ & Redundancy & + & Reliance on Experts & $\begin{array}{l}\stackrel{0}{0} \\
\dot{O} \\
ن\end{array}$ & 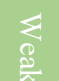 & 这 & 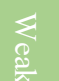 & 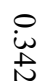 & $\sum_{0}^{£}$ & - & - & - & is & is & i \\
\hline ని & Redundancy & + & Responses (Actions) Repertoire & $\begin{array}{l}\stackrel{\circ}{0} \\
\dot{O}\end{array}$ & 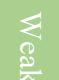 & 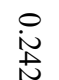 & 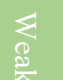 & : & 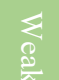 & - & - & - & is & is & i \\
\hline ¿ & Redundancy & - & $\begin{array}{l}\text { Obscuring and Neglecting Significant } \\
\text { Information }\end{array}$ & : & $\underset{\substack{1 \\
0}}{\frac{1}{2}}$ & i & $\underset{\frac{1}{2}}{\frac{8}{\pi}}$ & i⿱宀⿻心㇒ & 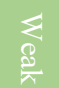 & - & - & - & $\begin{array}{c}\text { i } \\
\text { is }\end{array}$ & i & i \\
\hline$\stackrel{8}{\risingdotseq}$ & Redundancy & - & Normalization & $\begin{array}{l}\stackrel{0}{0} \\
\stackrel{2}{2}\end{array}$ & 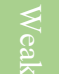 & 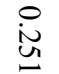 & $\begin{array}{l}\xi \\
\mathbb{2} \\
\frac{2}{2}\end{array}$ & 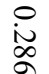 & $\stackrel{2}{8}$ & - & - & - & is & 岕 & is \\
\hline ن̊ & Relative Expertise & + & DEFERENCE TO EXPERTISE & : & 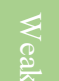 & ì & 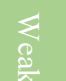 & $\stackrel{\frac{\pi}{\sigma}}{a}$ & 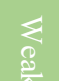 & - & - & - & is & is & ט \\
\hline
\end{tabular}




\begin{tabular}{|c|c|c|c|c|c|c|c|c|c|c|c|c|c|c|c|}
\hline ฉ & Relative Expertise & + & Responses (Actions) Repertoire & $\stackrel{\circ}{\dot{0}}$ & 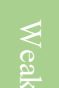 & 芯 & $\underset{0}{\approx}$ & 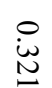 & 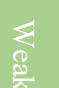 & - & - & - & i & $i_{\omega}$ & is \\
\hline is & Relative Expertise & + & Redundancy & $\stackrel{\circ}{\circ}$ & $\underset{0}{\xi}$ & $\underset{ \pm}{\stackrel{ \pm}{ \pm}}$ & $\underset{\substack{\infty \\
\frac{\delta}{\alpha}}}{\sum}$ & : & $\underset{\frac{8}{\alpha}}{\frac{8}{\alpha}}$ & - & - & - & is & $i_{\omega}$ & $i_{\omega}$ \\
\hline$\infty$ & Reliance on Experts & - & Members' Level of Participation & $\stackrel{\circ}{\circ}$ & 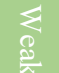 & $\begin{array}{l}0 \\
\text { i্. }\end{array}$ & 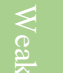 & 咐 & $\underset{\substack{\alpha \\
\frac{d}{x}}}{2}$ & - & - & - & i & i & is \\
\hline : & Reliance on Experts & - & DEFERENCE TO EXPERTISE & 通 & 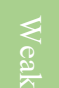 & 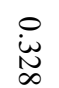 & 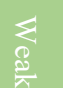 & $\stackrel{\circ}{\circ}$ & 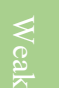 & - & - & - & ¿ & ¿ & $i_{\omega}$ \\
\hline$\overline{8}$ & Requisite Variety and Complexity & + & Organizing for Variety & $\stackrel{\dot{\omega}}{\underline{v}}$ & 里 & : & $\begin{array}{l}\frac{1}{0} \\
\frac{0}{0} \\
0 \\
03\end{array}$ & $\begin{array}{l}0 \\
\dot{\infty} \\
\text { 岕 }\end{array}$ & है & 0 & - & 0 & i & के & : \\
\hline 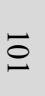 & Requisite Variety and Complexity & - & $\begin{array}{l}\text { Obscuring and Neglecting Significant } \\
\text { Information }\end{array}$ & $\stackrel{\circ}{\dot{\omega}}$ & 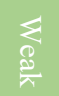 & i & $\underset{\substack{k \\
\mathbb{R}}}{\frac{1}{\pi}}$ & ì & 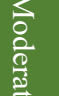 & - & 0 & 0 & i & is & ஜे \\
\hline ก & Requisite Variety and Complexity & + & Listening to Various Voices and Inputs & $\dot{\partial}$ & $\sum_{\substack{\alpha \\
\frac{0}{1}}}^{\infty}$ & $\stackrel{\ominus}{\ominus}$ & $\begin{array}{l}\sum \\
\mathbb{8} \\
\frac{\vec{\theta}}{\lambda}\end{array}$ & $\begin{array}{l}\dot{0} \\
\dot{u} \\
\dot{\omega}\end{array}$ & 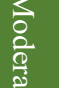 & - & 0 & 0 & i & is & aे \\
\hline
\end{tabular}

$\omega$ 


\begin{tabular}{|c|c|c|c|c|c|c|c|c|c|c|c|c|c|c|c|}
\hline $\bar{\omega}$ & Requisite Variety and Complexity & + & Distinctions & \begin{tabular}{l}
0 \\
$\dot{0}$ \\
0 \\
\hdashline
\end{tabular} & 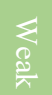 & $\underset{\dot{\omega}}{\dot{0}}$ & $\underset{\substack{\infty \\
\frac{0}{\pi}}}{\gtrless}$ & 耑 & 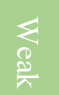 & - & - & - & is & i & i \\
\hline$\vec{\perp}$ & Requisite Variety and Complexity & - & Normalization & $\begin{array}{l}\stackrel{0}{0} \\
\dot{1}\end{array}$ & 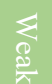 & 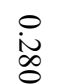 & $\begin{array}{l}\underset{\alpha}{\alpha} \\
\frac{d}{x}\end{array}$ & 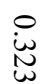 & 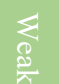 & - & - & - & $\dot{\omega}_{\omega}$ & i & i \\
\hline \& & Requisite Variety and Complexity & + & Responses (Actions) Repertoire & $\dot{8}$ & 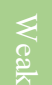 & 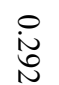 & $\underset{\frac{1}{\alpha}}{\frac{2}{x}}$ & $\dot{\sim}_{\tilde{\omega}}$ & 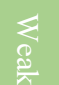 & - & - & - & $\begin{array}{c}\stackrel{0}{i} \\
\text { is }\end{array}$ & i & i \\
\hline ฉ̊ & Requisite Variety and Complexity & + & Redundancy & 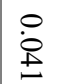 & 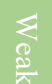 & $\underset{\dot{\omega}}{\stackrel{\dot{\omega}}{\nu}}$ & $\underset{\frac{8}{x}}{\sum}$ & 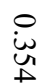 & 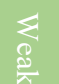 & - & - & - & $\stackrel{\omega}{\omega}$ & i & i \\
\hline 어 & Requisite Variety and Complexity & + & Precaution as a Mind-Set & : & 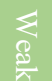 & $\dot{\mathrm{w}}_{\omega}$ & 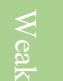 & $\begin{array}{l}\text { in } \\
\text { 怨 }\end{array}$ & $\begin{array}{l}\frac{1}{0} \\
\frac{0}{0}\end{array}$ & - & 0 & 0 & is & is & ஜे \\
\hline$\infty$ & Requisite Variety and Complexity & + & $\begin{array}{l}\text { Action Interpretation and Recursive } \\
\text { Interaction }\end{array}$ & : & $\underset{\substack{\infty \\
\frac{0}{\pi}}}{\frac{1}{\pi}}$ & $\dot{i}_{\dot{w}}$ & 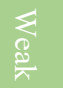 & 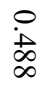 & $\begin{array}{l}\vec{\circ} \\
\stackrel{0}{0} \\
\stackrel{0}{0}\end{array}$ & - & 0 & 0 & is & is & ஜ̊̀ \\
\hline 8 & Requisite Variety and Complexity & + & Organizing for Sense-Discrediting & 啰 & 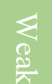 & $\begin{array}{l}\text { : } \\
\text { i্ }\end{array}$ & $\underset{\frac{\delta}{\alpha}}{\frac{1}{\alpha}}$ & 离 & 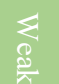 & - & - & - & i & i & o \\
\hline
\end{tabular}




\begin{tabular}{|c|c|c|c|c|c|c|c|c|c|c|c|c|c|c|c|}
\hline$\Xi$ & Requisite Variety and Complexity & + & $\begin{array}{l}\text { Anticipating and Controlling Latent } \\
\text { Singles }\end{array}$ & : & 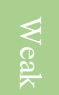 & $\begin{array}{l}\stackrel{0}{0} \\
\stackrel{+}{\omega}\end{array}$ & 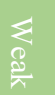 & \begin{tabular}{l}
0 \\
: \\
\multirow{0}{0}{}
\end{tabular} & 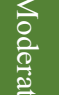 & - & 0 & 0 & is & is & ஜे \\
\hline$\Xi$ & Respectful Yielding & + & DEFERENCE TO EXPERTISE & $\begin{array}{l}\circ \\
\dot{\infty} \\
\dot{0}\end{array}$ & $\sum_{\substack{x \\
\frac{1}{1}}}^{\infty}$ & 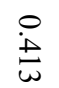 & $\underset{\substack{1 \\
0}}{\frac{1}{\pi}}$ & $\frac{0}{8}$ & $\sum_{\substack{\pi \\
\frac{1}{2}}}^{2}$ & - & - & - & $\dot{\omega}_{\omega}$ & $i_{\omega}$ & i \\
\hline$\overline{\tilde{N}}$ & Responses (Actions) Repertoire & + & COMMITMENT TO RESILIENCE & : & 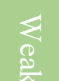 & i & $\sum_{\substack{1 \\
\frac{1}{x}}}^{\infty}$ & i & $\sum_{\substack{0 \\
\infty}}^{\infty}$ & - & - & - & i & is & is \\
\hline $\bar{\omega}$ & Responses (Actions) Repertoire & + & Improvisation & : & 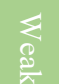 & $\underset{\sim}{\stackrel{\sim}{\sim}}$ & 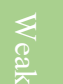 & 离 & 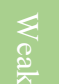 & - & - & - & $\stackrel{\omega}{\omega}$ & i & i \\
\hline$\vec{\perp}$ & Responses (Actions) Repertoire & + & $\begin{array}{l}\text { Anticipating and Controlling Latent } \\
\text { Singles }\end{array}$ & : & $\underset{\substack{\infty \\
\frac{0}{\pi}}}{\frac{0}{\pi}}$ & $\underset{\sim}{\dot{N}}$ & $\sum_{\frac{1}{2}}^{\sum}$ & $\underset{\substack{\dot{\infty} \\
\infty}}{\substack{\infty \\
0}}$ & $\begin{array}{l}\sum_{\infty}^{\infty} \\
\frac{D}{\pi}\end{array}$ & - & - & - & is & is & i \\
\hline ঢ̆ & Responses (Actions) Repertoire & + & $\begin{array}{l}\text { Sustaining Dynamic Situational } \\
\text { Awareness }\end{array}$ & $\stackrel{\grave{O}}{\infty}$ & $\underset{\substack{k \\
\frac{d}{\pi}}}{\frac{d}{\pi}}$ & $\dot{i}_{\tilde{u}}$ & 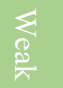 & $\underset{\sim}{\stackrel{\sim}{\sim}}$ & $\begin{array}{l}\sum \\
\substack{\infty \\
\frac{D}{2}}\end{array}$ & - & - & - & is & i & i \\
\hline ๙ & Responses (Actions) Repertoire & + & Sensemaking & $\stackrel{\circ}{\stackrel{3}{3}}$ & 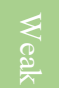 & 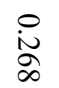 & $\underset{\substack{\infty \\
\frac{8}{2}}}{2}$ & $\underbrace{\stackrel{i}{u}}_{u}$ & $\sum_{\substack{n \\
\frac{1}{2}}}^{\infty}$ & - & - & - & i & ¿ & is \\
\hline
\end{tabular}




\begin{tabular}{|c|c|c|c|c|c|c|c|c|c|c|c|c|c|c|c|}
\hline$\Xi$ & Self-Awareness & + & Respectful Yielding & $\stackrel{\circ}{\Xi}$ & $\begin{array}{l}\sum \\
\mathbb{1} \\
\frac{B}{x}\end{array}$ & i & $\begin{array}{l}\sum \\
\frac{8}{2}\end{array}$ & 㒸 & 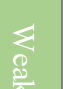 & - & - & - & i & is & is \\
\hline$\vec{\infty}$ & Self-Awareness & + & Relative Expertise & $\stackrel{\circ}{\dot{\infty}}$ & 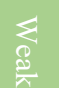 & $\stackrel{\infty}{\infty}_{\substack{\infty \\
u}}^{\infty}$ & $\sum_{\substack{1 \\
\frac{1}{\pi}}}^{2}$ & $\underset{\substack{\infty \\
\infty \\
\infty}}{\stackrel{\infty}{\infty}}$ & 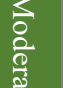 & - & 0 & 0 & i & i & ஷे \\
\hline $\bar{\sigma}$ & Self-Interest & - & Relative Expertise & $\dot{8}$ & 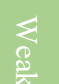 & $\begin{array}{l}\text { in } \\
\text { Ñ }\end{array}$ & 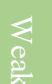 & 芯 & 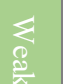 & - & - & - & i & ¿ & 出 \\
\hline$\overline{0}$ & Self-Interest & - & Respectful Yielding & $\dot{\ddot{Q}}_{\mathscr{L}}$ & $\begin{array}{l}\sum \\
\frac{8}{\pi}\end{array}$ & $\stackrel{\circ}{i}$ & $\sum_{\substack{\frac{3}{x} \\
\frac{3}{1}}}$ & $\dot{\mathscr{\omega}}_{\infty}$ & 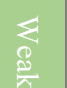 & - & - & - & is & ¿ & $i_{\omega}$ \\
\hline 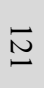 & Self-Interest & - & Heedful Interrelating & : & $\underset{\substack{k \\
\frac{1}{\pi}}}{\frac{2}{2}}$ & 응 & 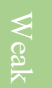 & $\stackrel{\circ}{\dot{\infty}}$ & $\begin{array}{l}\sum \\
\frac{D}{2}\end{array}$ & - & - & - & 岕 & i & $i$ \\
\hline N & Sensemaking & + & $\begin{array}{l}\text { Anticipating and Controlling Latent } \\
\text { Singles }\end{array}$ & $\stackrel{\circ}{\dot{0}}$ & $\underset{\substack{1 \\
\infty}}{\frac{1}{n}}$ & $\stackrel{\circ}{\stackrel{P}{ \pm}}$ & 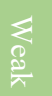 & 唸 & 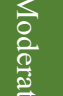 & - & 0 & 0 & is & i & ஷ் \\
\hline 心 & Sensemaking & + & COMMITMENT TO RESILIENCE & 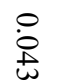 & 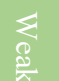 & $\stackrel{i}{u}_{0}$ & 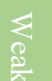 & $\underset{\underset{N}{\sim}}{\stackrel{0}{0}}$ & 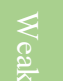 & - & - & - & 岕 & 这 & is \\
\hline
\end{tabular}




\begin{tabular}{|c|c|c|c|c|c|c|c|c|c|c|c|c|c|c|c|}
\hline$\stackrel{\bar{\perp}}{\square}$ & Sensemaking & + & $\begin{array}{l}\text { Action Interpretation and Recursive } \\
\text { Interaction }\end{array}$ & : & $\sum_{\substack{x \\
\frac{0}{1}}}^{\sum}$ & 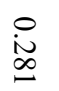 & $\begin{array}{l}\sum \\
\frac{1}{x} \\
\frac{2}{1}\end{array}$ & $\underset{\substack{\infty \\
\infty}}{\infty}$ & $\underset{\substack{1 \\
\infty}}{\frac{1}{\pi}}$ & - & - & - & i & i & i \\
\hline Nu & Sensemaking & + & Elasticity and Recovery & $\stackrel{\circ}{\circ}$ & $\sum_{\substack{x \\
\frac{d}{x}}}^{2}$ & $\underset{\text { i }}{\text { i }}$ & $\sum_{\substack{x \\
\frac{1}{x}}}^{\sum}$ & $\underset{\substack{\sim \\
\sim}}{i}$ & $\sum_{\substack{\infty \\
\frac{8}{x}}}^{\sum}$ & - & - & - & $i_{\omega}^{0}$ & i & i \\
\hline $\bar{\kappa}$ & Simple Structure & + & Improvisation & $\dot{8}$ & $\sum_{\substack{x \\
\frac{1}{x}}}$ & $\underset{i}{i}$ & $\sum_{\substack{x \\
\frac{1}{x}}}^{\infty}$ & : & 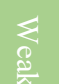 & - & - & - & is & i & i \\
\hline $\bar{N}$ & Structure Flexibility & + & DEFERENCE TO EXPERTISE & i & 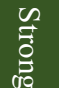 & 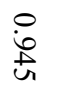 & 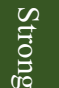 & : & 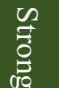 & - & - & - & $\dot{8}$ & $\dot{8}$ & $\dot{8}$ \\
\hline$\underset{\infty}{\bar{N}}$ & $\begin{array}{l}\text { Sustaining Dynamic Situational } \\
\text { Awareness }\end{array}$ & + & Operations as an Integrated Map & $\stackrel{\stackrel{\circ}{+}}{\stackrel{+}{+}}$ & 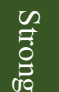 & 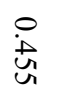 & 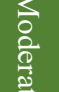 & $\stackrel{0}{\stackrel{U}{J}}$ & 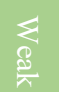 & 0 & 0 & 0 & $\dot{8}$ & ஜे & $\omega$ \\
\hline Nָ & $\begin{array}{l}\text { Sustaining Dynamic Situational } \\
\text { Awareness }\end{array}$ & + & SENSITIVITY TO OPERATIONS & 官 & $\begin{array}{l}\overrightarrow{0} \\
\stackrel{0}{0} \\
\stackrel{0}{0}\end{array}$ & \begin{tabular}{l}
$\stackrel{8}{6}$ \\
\multirow{\sigma}{\sigma}{}
\end{tabular} & 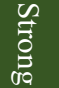 & : & 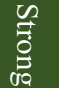 & 0 & 0 & - & के & i: & $\dot{8}$ \\
\hline $\bar{\sigma}$ & $\begin{array}{l}\text { Sustaining Dynamic Situational } \\
\text { Awareness }\end{array}$ & + & Sensemaking & : & 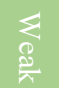 & 总 & 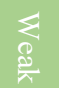 & 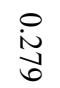 & $\sum_{\substack{1 \\
\infty}}^{\frac{\pi}{2}}$ & - & - & - & is & is & is \\
\hline
\end{tabular}

N 


\begin{tabular}{|c|c|c|c|c|c|c|c|c|c|c|c|c|c|c|c|}
\hline $\bar{\omega}$ & $\begin{array}{l}\text { Sustaining Dynamic Situational } \\
\text { Awareness }\end{array}$ & + & Interruptions and Adjustments & $\dot{\circ}$ & 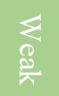 & $\stackrel{i}{u}_{\omega}$ & $\sum_{\substack{1 \\
\frac{1}{2}}}^{2}$ & $\stackrel{\circ}{\stackrel{i}{N}}$ & $\sum_{\frac{\infty}{\alpha}}^{\sum}$ & - & - & - & $\omega$ & i & \\
\hline$\underset{\sim}{\sim}$ & $\begin{array}{l}\text { Sustaining Dynamic Situational } \\
\text { Awareness }\end{array}$ & + & $\begin{array}{l}\text { Anticipating and Controlling Latent } \\
\text { Singles }\end{array}$ & $\stackrel{\circ}{\stackrel{\perp}{\perp}}$ & $\begin{array}{l}\sum \\
\frac{8}{2} \\
\frac{8}{n}\end{array}$ & $\underset{i}{i}$ & $\begin{array}{l}\sum \\
\frac{1}{2} \\
\frac{d}{n}\end{array}$ & $\stackrel{i}{\omega}_{\omega}^{\infty}$ & $\begin{array}{l}\sum \\
\substack{\pi \\
\frac{1}{2}}\end{array}$ & - & - & - & i & is & \\
\hline $\bar{\omega}$ & System Flexibility and Adaptability & + & COMMITMENT TO RESILIENCE & $\underset{\infty}{\stackrel{i}{\infty}}$ & 袋 & $\underset{+}{\stackrel{P}{+}}$ & $\sum_{0}^{\sum}$ & $\underset{\dot{\omega}}{v}$ & 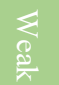 & 0 & 0 & - & 8 & i & \\
\hline
\end{tabular}


Appendix G: Materials of the Contextualization Procedure

Table 35: Scenario Based Managerial Interventions

\begin{tabular}{|c|c|c|}
\hline Intervention (Source) & $\rightarrow$ & HRO-Relevant Concepts (Target) \\
\hline \multirow{8}{*}{ Stop-Work-Authority } & + & Adherence to Standards and Risk Boundaries \\
\hline & - & Dysfunctional Momentum \\
\hline & - & Pressure \\
\hline & + & Level of Employee Participation \\
\hline & + & Personal Competence and Confidence \\
\hline & + & Simple Structure \\
\hline & + & Structural Flexibility \\
\hline & + & System Flexibility and Adaptability \\
\hline \multirow{5}{*}{$\begin{array}{l}\text { Reporting Near Misses and Safety-Critical } \\
\text { Events }\end{array}$} & + & Adherence to Standards and Risk Boundaries \\
\hline & - & Dysfunctional Momentum \\
\hline & + & Learning from Failure/Incidents \\
\hline & + & Level of Employee Participation \\
\hline & + & Organizing for Action-Based Inquiry \\
\hline \multirow{8}{*}{ Fostering Social Ties and Mutual Respect } & - & Ignoring True Expertise \\
\hline & + & Level of Employee Participation \\
\hline & + & Organizing for Action-Based Inquiry \\
\hline & + & Personal Competence and Confidence \\
\hline & + & Organizational Variety \\
\hline & + & Redundancy \\
\hline & + & Self-Awareness \\
\hline & - & Self-Interest \\
\hline & + & Level of Employee Participation \\
\hline & - & Overreliance on Experts \\
\hline
\end{tabular}




\begin{tabular}{|c|c|c|}
\hline \multirow[t]{2}{*}{ Institutionalizing Prosocial Motivation } & + & Self-Awareness \\
\hline & - & Self-Interest \\
\hline \multirow{6}{*}{$\begin{array}{l}\text { Foster a Sense of Personal Accountability for } \\
\text { Safety on All Levels }\end{array}$} & + & Adherence to Standards and Risk Boundaries \\
\hline & - & Complacency \\
\hline & - & Ignoring True Expertise \\
\hline & + & Level of Employee Participation \\
\hline & - & Self-Interest \\
\hline & + & Self-Awareness \\
\hline \multirow{6}{*}{ Implementing Safety Huddles } & + & Adherence to Standards and Risk Boundaries \\
\hline & + & Learning from Failure/Incidents \\
\hline & + & Level of Employee Participation \\
\hline & + & Organizing for Action-Based Inquiry \\
\hline & + & Personal Competence and Confidence \\
\hline & + & Organizational Variety \\
\hline \multirow{6}{*}{$\begin{array}{l}\text { Practicing Tabletop Exercises } \\
\text { Practicing Post-Event Debriefings } \\
\text { Practicing Emergency Drills }\end{array}$} & + & Adherence to Standards and Risk Boundaries \\
\hline & + & Domain-Specific Experience \\
\hline & + & Learning from Failure/Incidents \\
\hline & + & Level of Employee Participation \\
\hline & + & Organizing for Action-Based Inquiry \\
\hline & + & Personal Competence and Confidence \\
\hline \multirow{6}{*}{ Institutionalizing Emotional Ambivalence } & + & Adherence to Standards and Risk Boundaries \\
\hline & + & Level of Employee Participation \\
\hline & - & Overreliance on Experts \\
\hline & + & Personal Competence and Confidence \\
\hline & - & Self-Interest \\
\hline & + & Self-Awareness \\
\hline
\end{tabular}




\begin{tabular}{|c|c|c|}
\hline \multirow{7}{*}{ Managing Reliability Drift } & + & Adherence to Standards and Risk Boundaries \\
\hline & + & Staying Focused \\
\hline & - & Dysfunctional Momentum \\
\hline & + & Level of Employee Participation \\
\hline & + & Personal Competence and Confidence \\
\hline & - & Pressure \\
\hline & + & System Flexibility and Adaptability \\
\hline \multirow{6}{*}{ Practicing Individual Mindfulness } & + & Adherence to Standards and Risk Boundaries \\
\hline & + & Staying Focused \\
\hline & - & Loss of Situational Awareness \\
\hline & - & Loss of Task Attention \\
\hline & + & Level of Employee Participation \\
\hline & + & Personal Competence and Confidence \\
\hline \multirow{8}{*}{ Implementing Just-In-Time Learning } & + & Adherence to Standards and Risk Boundaries \\
\hline & + & Staying Focused \\
\hline & - & Loss of Situational Awareness \\
\hline & + & Level of Employee Participation \\
\hline & + & Personal Competence and Confidence \\
\hline & + & Understanding the Situation in Real-Time \\
\hline & + & Organizing for Action-Based Inquiry \\
\hline & + & Learning from Failure/Incidents \\
\hline
\end{tabular}




\section{Appendix H: Material and Outcomes of the Pilot Study}

\section{Team 1 Handout and Inputs}

\section{Encouraging Stop-Work Authority}

Definition: Employees and contractors are trained to stop work on an activity if, in their opinion, a continuation of the work poses a threat to human health, property, or the environment. This extends to stop-work cases that are not covered by government or company regulations. People are celebrated for stopping work, even if service is disrupted. Encouragement of stop-work authority is particularly emphasized when rank (line workers, contractors) or conditions (layoffs and economic worries, high production pressure) make it likely that people fail to stop work.

\section{How does implementing this intervention impact the following concepts?}

\section{Adherence to Standards and Risk Boundaries (+)}

Everybody is committed to precisely articulated standards for procedures and acceptable risk levels, despite the tendency of such commitments to erode over time. Procedural standards are protocols, checklists, responsibilities, job descriptions, required qualifications, rules of teamwork, etc. Risk boundaries define what is considered a risk and what is not, and which strategies are to be used to reduce or mitigate risks. They are the result of systematic risk assessments.

\section{Dysfunctional Momentum (-)}

Continuing in the course of action without stopping and reassessing the situation in total, particularly when the operations suffer lapses and unnoticed cues.

\section{Members Level of Participation (+)}


Levels of participation are determined by three factors: the level of involvement of employees in defining goals and ways to achieve them (contribution), the degree to which employees feel that their contributions are valued and used (representation), and the degree to which they focus on what the organization needs, even if this complicates their job.

\section{Pressure (-)}

Forces that can limit cognition, cause distraction, impede judgment, such as work and production pressure, time pressure.

\section{Personal Competence and Confidence ( + )}

Employees have the qualifications to improvise when needed successfully. This requires two things:

- General knowledge of:

- the system (concepts, boundaries, behavior) to predict its state

- possible actions to manage the system (knowledge of rules, regulations, standards, resources).

- Personal skills and confidence, based on knowing oneself, and having received encouragement, motivation and trust by upper management.

\section{Simple Structure (+)}

Minimal but clear structure that provides experienced people a framework for making sense of a situation and directing themselves as to what to do. Consists of core values, a mission statement, and a few core regulations and rules of engagement. Enables improvisation.

\section{Structural Flexibility (+)}

Reducing hierarchical structure's rigidity to migrate decision-making to lower levels, particularly to those in frontlines during emergencies and serious threats. Decision-making 
can thus be structured ad-hoc based on the needs of the situation. Structural flexibility requires that members of the organization trust that, somewhere in the system, there is sufficient competence to handle any problem and that it will be located when it is needed.

\section{System Flexibility and Adaptability (+)}

Ability to absorb shocks/disruptions without breaking (i.e. maintaining the same output) and to quickly recover if severe disruptions occur.

Table 36: Workshop: Team 1 Data Inputs

\begin{tabular}{|l|c|c|c|}
\hline Safety-Related Practices & $\begin{array}{c}\text { Expected } \\
\text { Impact }\end{array}$ & $\begin{array}{c}\text { Participants } \\
\text { Impact }\end{array}$ & Scale \\
\hline Adherence to Standards and Risk Boundaries & + & - & 0.63 \\
\hline Dysfunctional Momentum & + & + & 0.33 \\
\hline Member's level of participation & - & - & 0.33 \\
\hline Pressure & + & - & 0.33 \\
\hline Personal Competence and Confidence & + & + & 0.63 \\
\hline System Flexibility and Adaptability & + & + & 0.63 \\
\hline Simple Structure & + & + & 0.63 \\
\hline Structure Flexibility & + & + \\
\hline
\end{tabular}

Based on your experience, do you think this intervention has additional impacts on safety in offshore oil and gas? Please list them.

What are the problems that you experience or anticipate with this intervention?

\section{Team 2 Handout and Inputs}

Encouraging the Reporting of Near Misses and Safety-Critical Events 
Definition: Management actively encourages the reporting of near misses and safetycritical events and reduces barriers to reporting. Possible approaches: (1) clear definitions of what constitutes a reportable incident, (2) supervisor example (i.e., supervisors report themselves), (2) reporting as an expectation in performance evaluation, (3) immediate positive feedback for the reporting employee, pointing out the problem that may have been avoided as a result of him/her taking action, (4) no blame or fear of punishment for the reporting employee, (5) if the level of reporting is generally low: honorable mention or other visible (but not financially important) rewards for units which are top reporters, (6) After corrective action is taken, inform the reporting employee about it, (7) where appropriate, publicize the report and the corrective action beyond the immediately affected organizational unit.

\section{How does implementing this intervention impact the following concepts?}

\section{Adherence to Standards and Risk Boundaries (+)}

Everybody is committed to precisely articulated standards for procedures and acceptable risk levels, despite the tendency of such commitments to erode over time. Procedural standards are protocols, checklists, responsibilities, job descriptions, required qualifications, teamwork rules, etc. Risk boundaries define what is considered a risk and what is not, and which strategies are to be used to reduce or mitigate risks. They are the result of systematic risk assessments.

\section{Dysfunctional Momentum (-)}

Continuing in the course of action without stopping and reassessing the situation in total, particularly when the operations suffer lapses and unnoticed cues. 


\section{Learning from Failure/Incidents (+)}

A systematic process through which employees and the organization seek to understand any adverse safety events that have taken place to prevent similar future events. Decisionmakers involved in the incident receive feedback for their own decisions. People not involved in the incidents receive lessons learned to spread the knowledge gained.

\section{Members Level of Participation ( + )}

Levels of participation are determined by three factors: the level of involvement of employees in defining goals and ways to achieve them (contribution), the degree to which employees feel that their contributions are valued and used (representation), and the degree to which they focus on what the organization needs, even if this complicates their job.

\section{Organizing for Action-Based Inquiry (+)}

Rather than processing readily available information only, systems are set up so that employees can take action to seek out additional information actively. This can lead employees to re-interpret already existing information, possibly triggering further inquiries. Actions can include asking an expert, collecting other data, running an experiment, using data analytics, etc.).

Table 37: Workshop: Team 2 Data Inputs

\begin{tabular}{|l|c|c|c|}
\hline Concept & Expected Impact & Participant Impact & Scale \\
\hline Adherence to Standards and Risk Boundaries & + & + & 0.33 \\
\hline Dysfunctional Momentum & + & + & 0.33 \\
\hline Learning from Failure/Incidents & + & + & 0.63 \\
\hline Members' Level of Participation & + & + & 0.33 \\
\hline Organizing for Action-Based Inquiry & + & 0.33 \\
\hline
\end{tabular}


Based on your experience, do you think this intervention has additional impacts on safety in offshore oil and gas? Please list them.

What are the problems that you experience or anticipate with this intervention?

\section{Team 3 Handout and Inputs}

\section{Fostering Social Ties and Mutual Respect}

Definition: Creating a work environment that fosters social ties that build on mutual respect. Actions can include onboarding new team members (e.g., buddy or mentor system), creating opportunities for social interactions, and setting clear expectations for respectful behavior. This addresses the reliability challenges that occur when people do not know each other well enough. They do not interact frequently or intensively. They have modest mutual knowledge and move from one area or role to another while not collaborating over extended periods.

\section{How does implementing this intervention impact the following concepts?}

\section{Ignoring True Expertise (-)}

Failing to identify and leverage the knowledge of true experts. Reasons can be relying on rank, tenure, or perceived competence or "not invented here" (particularly after mergers).

\section{Members Level of Participation ( + )}

Levels of participation are determined by three factors: the level of involvement of employees in defining goals and ways to achieve them (contribution), the degree to which employees feel that their contributions are valued and used (representation), and the degree to which they focus on what the organization needs, even if this complicates their job.

\section{Organizing for Action-Based Inquiry ( + )}


Rather than processing readily available information only, systems are set up so that employees can take action to seek out additional information actively. This can lead employees to re-interpret already existing information, possibly triggering additional inquiries. Actions can include asking an expert, collecting additional data, running an experiment, using data analytics, etc.).

\section{Personal Competence and Confidence (+)}

Employees have the qualifications to improvise successfully, when needed. This requires two things:

- general knowledge of:

- the system (concepts, boundaries, behavior, ...) to predict its state

- possible actions to manage the system (knowledge of rules, regulations, standards, resources).

- Personal skills and confidence, based on knowing oneself, and having received encouragement, motivation, and trust by upper management.

\section{$\underline{\operatorname{Redundancy}(+)}$}

Designed duplications in the system for technological, individual, and organizational elements. This can include backup equipment and personnel (e.g., backup energy supplies, backup operator if the main operator becomes overwhelmed) and backup decision-making and control (e.g., having multiple people independently do the same control task).

\section{Requisite Variety and Complexity (+) (achieving the necessary organizational diversity)}

Decisions are made based on the inputs of people from varied functional, educational, and experiential backgrounds. This avoids simplistic interpretation and group-think. 
Disagreement and conflict are permitted and successfully negotiated, managed, and controlled. Variety can be designed through hiring practices, frequent and adversarial reviews, and by mixing up teams (e.g., job rotation and re-training and re-assignments).

\section{$\underline{\text { Self-Awareness (+) }}$}

It is referring to knowing and acknowledging the knowledge and the experiences of one's self, as well as the limits and gaps in them. (Self-awareness reduces resistance to asking for knowledge from others when needed).

Self-Interest (-) It refers to when members of the organization view their jobs based on their interests and define their work content solely on their roles and responsibilities officially given to them.

Table 38: Workshop: Team 3 Data Inputs

\begin{tabular}{|l|c|c|c|}
\hline Concept & Expected Impact & $\begin{array}{c}\text { Participant } \\
\text { Impact }\end{array}$ & Scale \\
\hline Ignoring True Expertise & - & - & 0.33 \\
\hline Members' Level of Participation & + & - & 0.33 \\
\hline Organizing for Action-Based Inquiry & + & + & 0.33 \\
\hline Personal Competence and Confidence & + & No Impact & 0.63 \\
\hline Requisite Variety and Complexity / (achieving \\
the necessary organizational diversity)
\end{tabular}




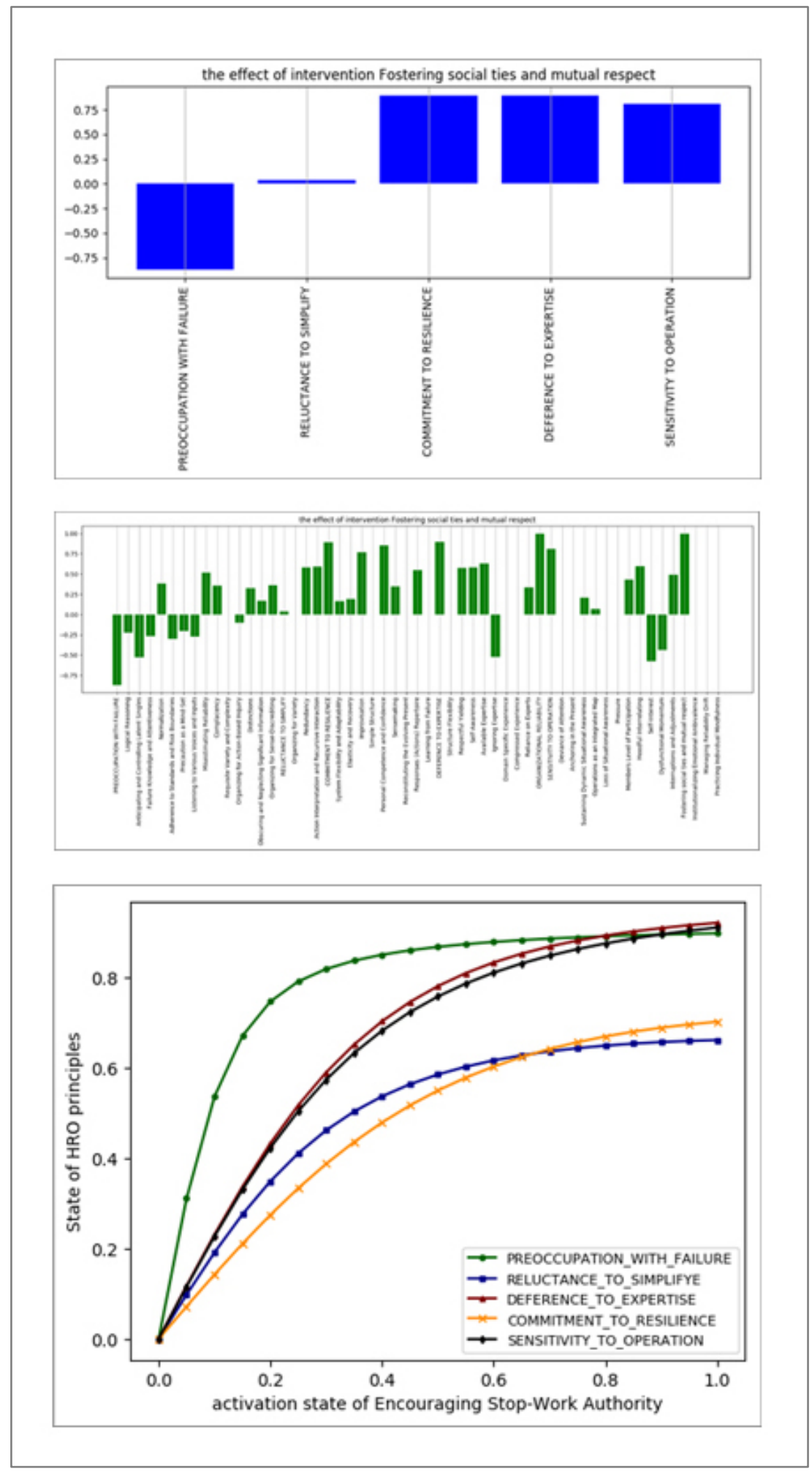

Figure 52: Model Simulation Results Based on Team 1 Inputs 


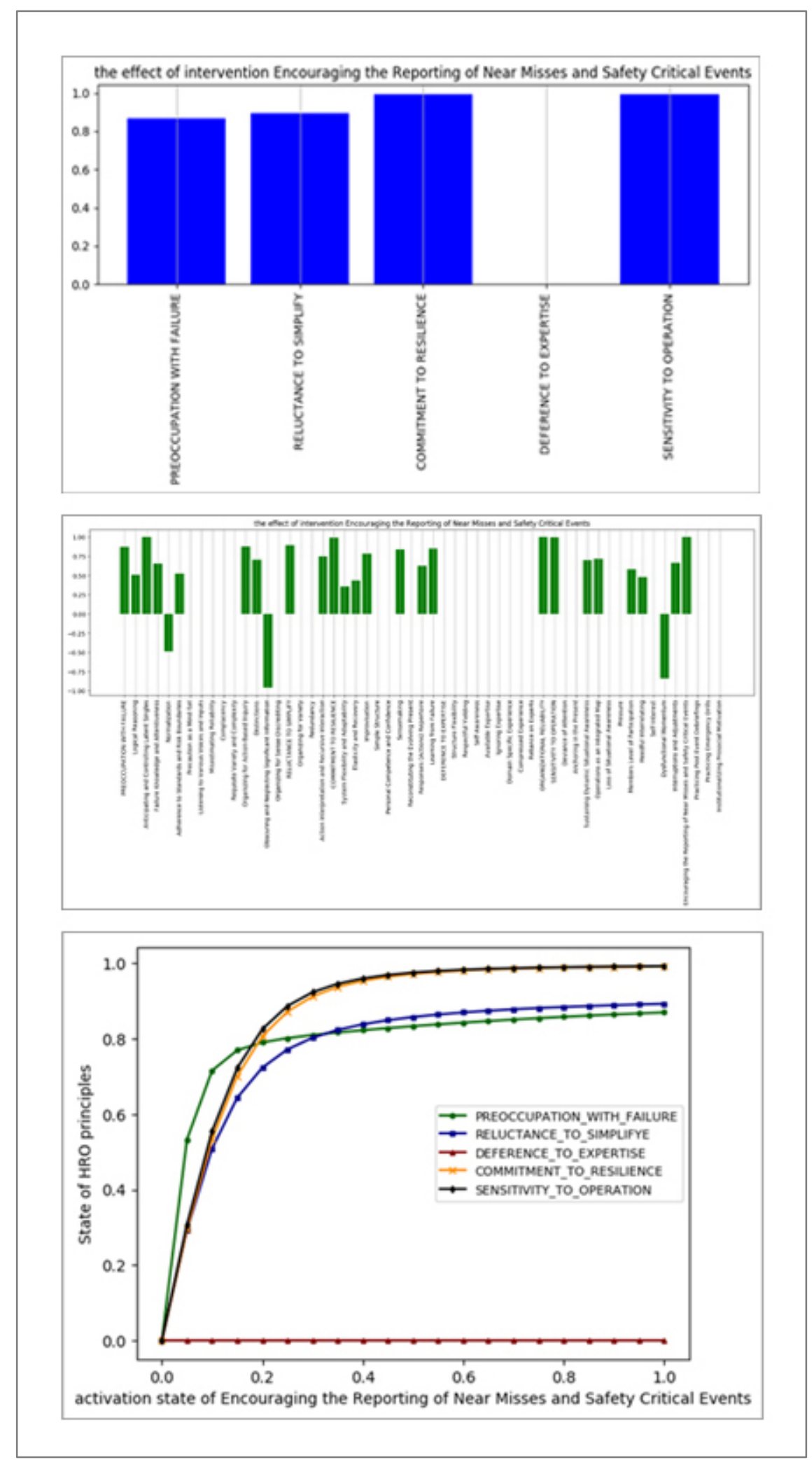

Figure 53: Model Simulation Results Based on Team 2 Inputs 

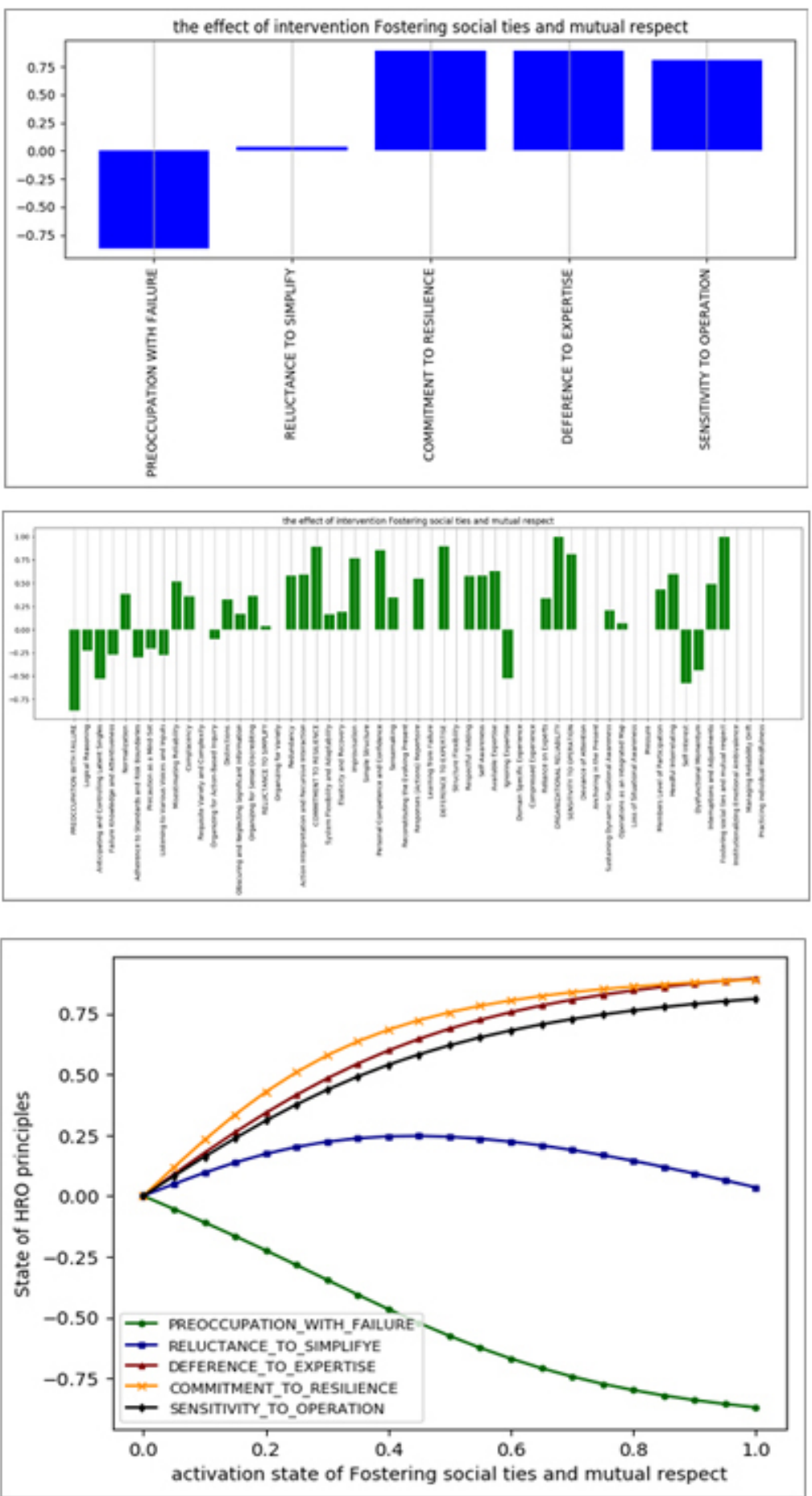

Figure 54: Model Simulation Results Based on Team 3 Inputs 


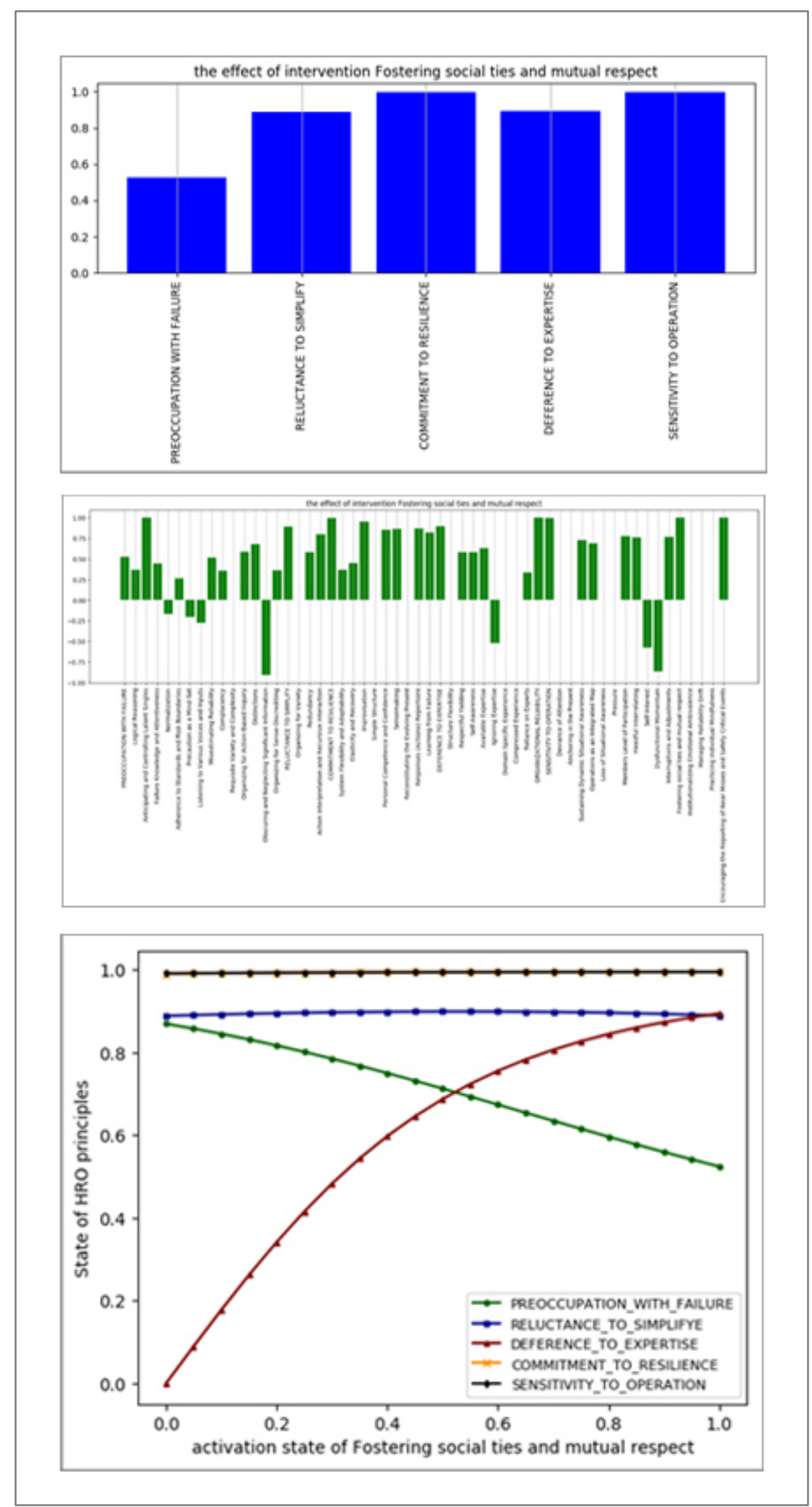

Figure 55: Model Simulation Results Based on Combining Team 2\&3 Inputs 


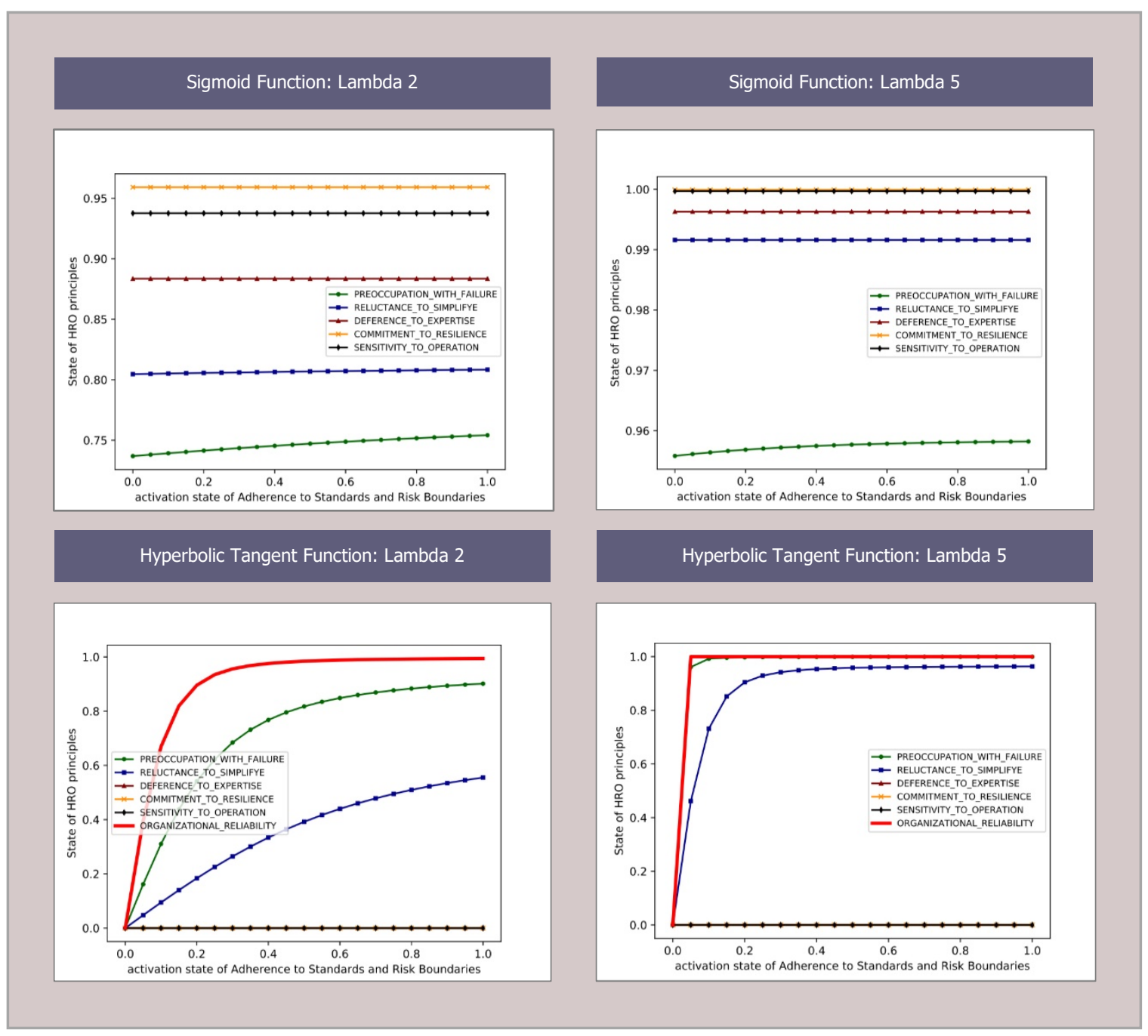

Figure 56: Different Inference Function and Two Values of Lambda $(2,5)$ 


\section{Appendix I: Online Survey Final Results}

Table 39 - Online Survey Final Results Calculated Based on the Median

\begin{tabular}{|c|c|c|}
\hline Intervention & HRO-Relevant Concepts & $\begin{array}{l}\text { Edge Sign and } \\
\text { Weight Based on } \\
\text { the Median }\end{array}$ \\
\hline \multirow{8}{*}{ Stop-Work Authority } & Adherence to Standard and Risk Boundaries & 0.67 \\
\hline & System flexibility and adaptability & -0.67 \\
\hline & Simple structure & -0.33 \\
\hline & Personal competence and confidence & 0.33 \\
\hline & Structure flexibility & -0.33 \\
\hline & Pressure & 0.33 \\
\hline & Level of employee participation & 0.33 \\
\hline & Dysfunctional momentum & -0.67 \\
\hline \multirow{5}{*}{$\begin{array}{l}\text { Reporting of Near Misses and } \\
\text { Safety-Critical Events }\end{array}$} & Adherence to Standard and Risk Boundaries & 1.00 \\
\hline & Level of employee participation & 0.67 \\
\hline & Learning from Failure/Incidents & 0.67 \\
\hline & Organizing for action-based inquiry & 0.33 \\
\hline & Dysfunctional momentum & 0.67 \\
\hline \multirow{8}{*}{$\begin{array}{c}\text { Fostering Social Ties and Mutual } \\
\text { Respect }\end{array}$} & Organizing for action-based inquiry & 0.67 \\
\hline & Organizational variety & 0.67 \\
\hline & Redundancy & -0.33 \\
\hline & Personal competence and confidence & 0.33 \\
\hline & Self-awareness & 0.67 \\
\hline & Ignoring expertise & 0.67 \\
\hline & Level of employee participation & 0.67 \\
\hline & Self-interest & -0.33 \\
\hline \multirow{7}{*}{$\begin{array}{c}\text { Foster a Sense of Personal } \\
\text { Accountability for Safety on All } \\
\text { Levels }\end{array}$} & Adherence to Standard and Risk Boundaries & 0.67 \\
\hline & Complacency & -0.67 \\
\hline & Learning from Failure/Incidents & 0.00 \\
\hline & Self-awareness & 0.67 \\
\hline & Ignoring expertise & 0.00 \\
\hline & Level of employee participation & 0.67 \\
\hline & Self-interest & 0.67 \\
\hline
\end{tabular}




\begin{tabular}{|c|c|c|}
\hline \multirow{6}{*}{ Implementing Safety Huddles } & Adherence to Standard and Risk Boundaries & 0.67 \\
\hline & Organizing for action-based inquiry & 0.33 \\
\hline & Organizational variety & 0.00 \\
\hline & Personal competence and confidence & 0.67 \\
\hline & Learning from Failure/Incidents & 0.67 \\
\hline & Level of employee participation & 0.67 \\
\hline \multirow{6}{*}{ Tabletop Exercises } & Adherence to Standard and Risk Boundaries & 0.33 \\
\hline & Organizing for action-based inquiry & 0.50 \\
\hline & Personal competence and confidence & 0.67 \\
\hline & Learning from Failure/Incidents & 0.50 \\
\hline & Domain Specific Experience & 0.50 \\
\hline & Level of employee participation & 0.17 \\
\hline \multirow{6}{*}{ Post-Event Debriefings } & Adherence to Standard and Risk Boundaries & 1.00 \\
\hline & Organizing for action-based inquiry & 1.00 \\
\hline & Personal competence and confidence & 1.00 \\
\hline & Learning from Failure/Incidents & 1.00 \\
\hline & Domain Specific Experience & 1.00 \\
\hline & Level of employee participation & 1.00 \\
\hline \multirow{6}{*}{ Practicing Emergency Drills } & Adherence to Standard and Risk Boundaries & 0.67 \\
\hline & Organizing for action-based inquiry & 0.67 \\
\hline & Personal competence and confidence & 0.67 \\
\hline & Learning from Failure/Incidents & 0.67 \\
\hline & Domain Specific Experience & 0.67 \\
\hline & Level of employee participation & 0.33 \\
\hline \multirow{5}{*}{ Prosocial Motivation } & Adherence to Standard and Risk Boundaries & 0.00 \\
\hline & Self-awareness & 0.67 \\
\hline & Overreliance on experts & 0.67 \\
\hline & Level of employee participation & 0.67 \\
\hline & Self-interest & 0.00 \\
\hline \multirow{5}{*}{ Emotional Ambivalence } & Adherence to Standard and Risk Boundaries & 0.50 \\
\hline & Personal competence and confidence & 0.67 \\
\hline & Self-awareness & 0.67 \\
\hline & Overreliance on experts & -0.67 \\
\hline & Level of employee participation & 0.67 \\
\hline
\end{tabular}




\begin{tabular}{|c|c|c|}
\hline & Self-interest & -0.33 \\
\hline \multirow{7}{*}{ Managing Reliability Drift } & Staying focused & 0.00 \\
\hline & Level of employee participation & 0.00 \\
\hline & Personal competence and confidence & 0.00 \\
\hline & Adherence to Standard and Risk Boundaries & 0.00 \\
\hline & System flexibility and adaptability & 0.00 \\
\hline & Pressure & -0.33 \\
\hline & Dysfunctional momentum & -0.33 \\
\hline \multirow{7}{*}{ Practicing Individual Mindfulness } & Adherence to Standard and Risk Boundaries & 0.33 \\
\hline & Personal competence and confidence & 0.67 \\
\hline & Loss of task attention & -0.50 \\
\hline & Staying focused & 0,67 \\
\hline & Pressure & -0.17 \\
\hline & Loss of situational awareness & -0.50 \\
\hline & Level of employee participation & 0.33 \\
\hline \multirow{9}{*}{ Just-In-Time Learning } & Adherence to Standard and Risk Boundaries & -0.67 \\
\hline & Organizing for action-based Inquiry & 0.00 \\
\hline & Personal competence and confidence & -0.33 \\
\hline & Understanding the situation in Realtime & -0.33 \\
\hline & Learning from Failure/Incidents & -0.67 \\
\hline & Loss of task attention & 0.33 \\
\hline & Staying focused & -0.67 \\
\hline & Loss of situational awareness & 0.33 \\
\hline & Level of employee participation & 0.33 \\
\hline
\end{tabular}

